\title{
Breitbandige \\ Ultraschallabsorptionsspektroskopie an wässrigen Kohlenhydrat-Lösungen
}

\author{
Dissertation \\ zur Erlangung des Doktorgrades \\ der Mathematisch-Naturwissenschaftlichen Fakultäten \\ der Georg-August-Universität zu Göttingen
}

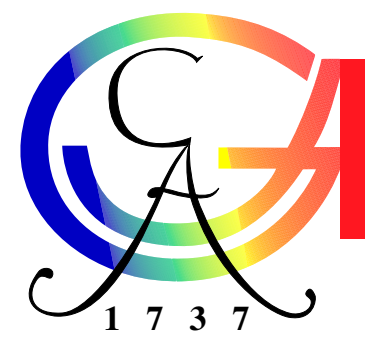
vorgelegt von
Ralf Hagen
aus
Uelzen

Göttingen 2003 
Referent:

Korreferent:

Tag der mündlichen Prüfung:
Prof. Dr. Dirk Ronneberger

Prof. Dr. Götz Eckold

14. November 2003 


\section{Inhaltsverzeichnis}

1 Einleitung 1

2 Grundlagen der Ultraschallabsorptionsspektroskopie 3

2.1 Ultraschalldämpfung . . . . . . . . . . . . . . . . . 5

2.1.1 Debye-Spektralfunktion . . . . . . . . . . . 7

3 Messverfahren 9

3.1 Das Resonatorverfahren . . . . . . . . . . . . . . . . . . 9

3.1 .1 Der ideale Resonator . . . . . . . . . . . . . . . . . . 9

3.1 .2 Der reale Resonator . . . . . . . . . . . . . . . . . . . . . . . 11

3.1.3 Blockschaltbild der Resonatormessplätze . . . . . . . . . . . . 15

3.1.4 Resonator-Messzellen . . . . . . . . . . . . . . . . . . . . 16

3.1 .5 Messablauf . . . . . . . . . . . . . . . . . . . 16

3.1 .6 Anpassrechnung . . . . . . . . . . . . . . . . . . . . . . . . . . 18

3.1 .7 Fehler der Resonatormessungen . . . . . . . . . . . . . . . . . 18

3.2 Das Schwingungspulstransmissionsverfahren . . . . . . . . . . . . 21

3.2.1 Blockschaltbild der Pulszellenmessplätze . . . . . . . . . . . . 21

3.2 .2 Grundlagen . . . . . . . . . . . . . . . . . . . 23

3.2.3 Schwingungspulsverfahren . . . . . . . . . . . . . . . 25

3.2.4 Bestimmung der Schallgeschwindigkeit . . . . . . . . . . . 25

3.2 .5 Empfängerkennlinie . . . . . . . . . . . . . . . . . . . . 26

3.2 .6 Beugungskorrektur . . . . . . . . . . . . . . . . . . . . . . . . . . . . . . . 26

3.2 .7 Messablauf . . . . . . . . . . . . . . . . . . . . . . . 28

3.2 .8 Schwingungspulstransmissions-Messzellen . . . . . . . . . . 28

3.2.9 Fehlerdiskussion für das Pulstransmissionsverfahren . . . . . . 34

4 Präparation der Messlösungen 37

5 Urea: Referenz für Ultraschallresonatormessungen 41

6 Kohlenhydrate $\quad 47$

6.1 Monosaccharide . . . . . . . . . . . . . . . . 47

6.1.1 Konstitution und Konfiguration von Monosacchariden . . . . . 48

6.1 .2 Ring-Ketten-Tautomerie . . . . . . . . . . . . . . 51

6.2 Disaccharide .................................. 54

6.2.1 Definition der glycosidischen Bindungswinkel $\phi, \psi$ und $\omega$. . . 54

6.2 .2 Maltose . . . . . . . . . . . . . . . . 58

6.2 .3 Trehalose . . . . . . . . . . . . . . . . . . . . . 58

6.2 .4 Lactose . . . . . . . . . . . . . . . . . . . . . . . . 58 
6.2 .5 Melibiose . . . . . . . . . . . . . . . . . . . 59

6.2 .6 Sucrose/Saccharose . . . . . . . . . . . . . . . . . 59

$\begin{array}{lll}7 \text { Auswertung } & \mathbf{6 1}\end{array}$

7.1 Disaccharide . . . . . . . . . . . . . . . . . . . . . . . 62

7.1 .1 Maltose . . . . . . . . . . . . . . . . 62

7.1 .2 Trehalose . . . . . . . . . . . . . . . . 66

7.1.3 Sucrose, Lactose und Melibiose . . . . . . . . . . . . . . . . 68

7.2 Kohlenhydrate mit Calcium . . . . . . . . . . . . . . . . 73

7.2 .1 Maltose . . . . . . . . . . . . . . . . . . 73

7.2 .2 Fructose . . . . . . . . . . . . . . . . 73

8 Diskussion $\quad 77$

8.1 Disaccharid-Spektren . . . . . . . . . . . . . . . . . . . 77

8.1 .1 Maltose . . . . . . . . . . . . . . . 77

8.2 Rotationsisomerie der exocyclischen Hydroxymethyl-Gruppe . . . . . 77

8.3 Der "Disaccharid" Prozess . . . . . . . . . . . . . . . . . . 83

8.3 .1 Sucrose . . . . . . . . . . . . . . . . . . . . . . . . . . . 88

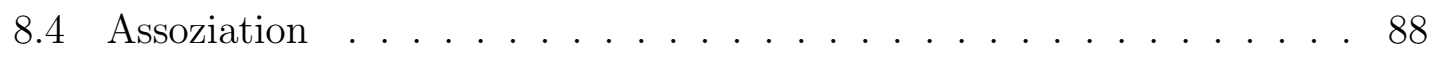

8.5 Kohlenhydrate mit Calciumzusatz . . . . . . . . . . . . . . . . . 91

8.5.1 Maltose und Glucose mit Calcium . . . . . . . . . . . . . . . . 91

8.5 .2 Fructose und Xylose . . . . . . . . . . . . . . . . . . . . . . . . 92

8.6 Aktivierungsenthalpie . . . . . . . . . . . . . . . . . 95

$\begin{array}{lll}9 & \text { Zusammenfassung und Ausblick } & 97\end{array}$

\begin{tabular}{ll}
\hline A Spektren einiger Monosaccharide & 99
\end{tabular}

A.1 Glucose . . . . . . . . . . . . . . . . . . . . . . . . . . . . . . 99

A.2 Fructose . . . . . . . . . . . . . . . . . . . . . . . . . . . . 99

A.3 Galactose . . . . . . . . . . . . . . . . . 100

Literaturverzeichnis I

Danksagung V V

Lebenslauf $\quad$ VII 


\section{Einleitung}

In den vergangenen Jahren hat die Forschung zu Kohlenhydraten einen regelrechten Boom erlebt. Lange Zeit galten die Kohlenhydrate, auch Saccharide und einfach Zucker genannt, im Vergleich zu Nukleotiden und Proteinen als wenig interessant für die „life sciences“ und wurden nur in ihrer Rolle als Energielieferanten und als Stützmaterial der Zellen wahrgenommen. So ist das Monosaccharid Glucose Hauptblutzucker bei Säugetieren, das Disaccharid Trehalose bei Insekten; Polysaccharide wie Glykoken und Stärke stellen Langzeitenergiespeicher dar. Ein weiteres Polysaccharid, die Zellulose, ist ein wichtiges Baumaterial für Pflanzenzellwände, und Chitin bildet das Außenskelett der Insekten und ist in Pilzen zu finden [7][36].

Darüber hinausgehend spielen Kohlenhydrate jedoch eine herausragende Rolle als Informationsträger bei Erkennungsprozessen auf zellulärer Ebene [7][3][8][6]. In eine typische Zellmembran sind eine Reihe verschiedener Glycolipide und Glycoproteine eingebettet, an die kurze, zum Teil verzweigte Oligosaccharidketten kovalent gebunden sind. Diese finden sich auf der extrazellulären Seite der Membran und bilden die sogenannte "Glycocalix“. An diese Kohlenhydrate kann eine Klasse von Proteinen, die Lektine bzw. Selektine, nach dem Schlüssel-Schloss Prinzip spezifisch und reversibel binden, wobei sie in der Lage sind kleinste Unterschiede in der Struktur der Oligosaccharide zu unterscheiden [1]. Diese selektiven Bindungseigenschaften mit Proteinen nutzt die Natur und verwendet Kohlenhydrate als universelle Markierung [3]. Sie sind verantwortlich für die Blutgruppenzugehörigkeit [7], bewirken die Adhäsion von Leukocyten im Bereich entzündeten Gewebes [4], spielen eine Rolle bei der Metastase von Tumorzellen [2] oder bei der Anheftung von Grippeviren an rote Blutkörperchen [1]. Kohlenhydrate sind als Informationsträger aufgrund ihrer schier unerschöpflichen Mannigfaltigkeit an Strukturen und Konformationen bestens geeignet. Amino- und Nucleinsäuren besitzen zwei Verknüpfungsstellen und können somit „nur" lineare Ketten bilden, während eine Hexopyranose, wie z.B. die Glucose, fünf Bindungsmöglichkeiten bietet, so dass mehrfach verzweigte Oligo- und Polysaccharide gebildet werden können. Berücksichtigt man weiterhin die Möglichkeit von $\alpha$ - oder $\beta$-glycosidischen Bindungen (siehe Kapitel 6), so sind bei einem Pentamer aus verschiedenen Monosacchariden bereits mehr als zwei Millionen Polysaccharide denkbar [6]. Somit liegt eine sehr kompakte und effiziente Informationsspeicherung durch Saccharide vor. Die Bindung der einzelnen Monosaccharide zu einem Oligosaccharid erfolgt über glycosidische Bindungen mit zwei oder drei nichtfixierten Einfachbindungen, so dass sich die Ausrichtung der einzelnen Ringe untereinander und damit die Konformation des gesamten Kohlenhydrates im thermischen Geschehen in Lösung ändern kann. Die kleinsten Oligosaccharide, in denen die charakteristischen glycosidischen Bindungen auftreten, sind die Disaccharide. So liegt in der Maltose eine $\alpha(1,4)$-glycosidische Bindung mit zwei nichtfixierten Einfachbindungen zwischen zwei Glucosemolekülen vor. In der Lactose findet sich die $\beta(1,4)$-glycosidische Bindung zwischen Galactose und Glucose. Aus diesen be- 


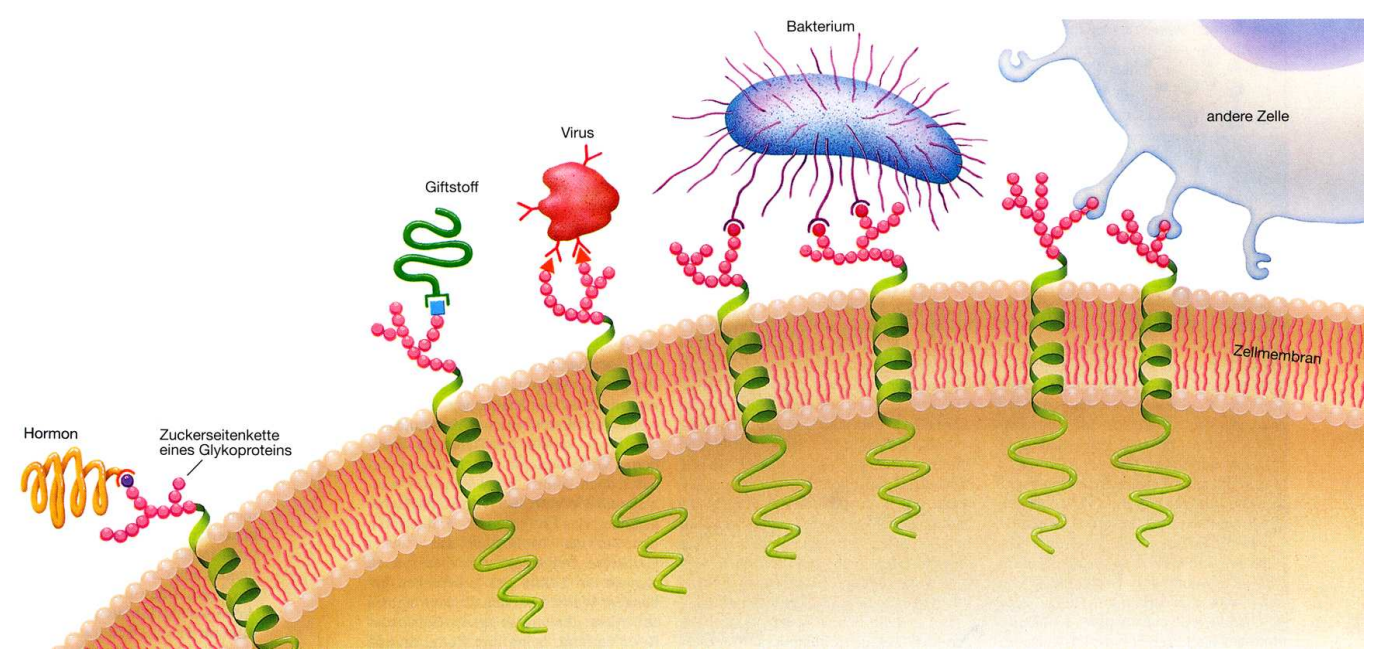

Abbildung 1.1: Beispiele für die Bedeutung von Kohlenhydraten auf der Oberfläche von Zellen [7].

steht auch die Melibiose, allerdings sind die Pyranoseringe hier $\alpha(1,6)$-glycosidisch gebunden, so dass drei frei rotierende Einfachbindungen zwischen den Monosaccharideinheiten vorliegen. In dieser Arbeit soll anhand verschiedener Disaccharide die Dynamik der Konformationsänderungen bei Rotation um die glycosidischen Einfachbindungen in wässrigen Lösungen untersucht werden. Hierbei wird zum Nachweis der dynamischen Prozesse die akustische Spektroskopie eingesetzt. Die mit der Schallwelle verbundenen lokalen Schwankungen des Druckes und der Temperatur koppeln über das Reaktionsvolumen und die Reaktionsenthalpie an das Gleichgewicht der verschiedenen Konformationen. Die Störung der inneren Freiheitsgrade hat Relaxationsphänomene zur Folge, die sich im spektralen Verlauf der Absorption widerspiegeln können. Die untersuchten Kohlenhydratlösungen müssen dabei nicht mit zusätzlichen Markern versehen werden, die die Dynamik solch kleiner Moleküle merklich beeinflussen würden.

Zusätzlich zu den reinen Disaccharidlösungen wurde auch die Wechselwirkung der Kohlenhydrate mit Calcium-Ionen untersucht. Calcium spielt eine wichtige Rolle bei der Nervenreizleitung, da calciumgesteuerte Kanäle die Exocytose und damit den Ausstoß von Neurotransmittern in der Synapse auslösen [5]. Über calciumgesteuerte Lektine hat es direkten Einfluss auf Saccharid-Proteinwechselwirkung [7]. Außerdem spielt Interaktion von Polysacchariden und Calcium-Ionon bei der Stabilität von Alginaten eine wichtige Rolle. Erst die Quervernetzung über Ionen, insbesondere $\mathrm{Ca}^{2+}$ und $\mathrm{Sr}^{2+}$ macht deren Stabilität möglich („egg-box-junctions“) [36]. 


\section{Grundlagen der Ultraschallabsorp- tionsspektroskopie}

Ziel dieser Arbeit ist es, mittels der Ultraschallspektroskopie schnelle molekulare Abläufe in Flüssigkeiten bzw. Lösungen zu untersuchen. Die Messung des Spektrums des Absorptionskoeffizienten $\alpha$ bietet eine Möglichkeit der Sondierung der Molekülanordnung und Dynamik, die sich im Wechselspiel mit einer Reihe weiterer Messmethoden befindet, welche spezifische Eigenschaften des jeweiligen Moleküls nutzen (Tabelle 2.1). Die Ultraschallwelle koppelt über das molekulare Volumen oder

\begin{tabular}{|l|l|l|}
\hline Molekül-,,Marke“ & „Sonde“6 & Wirkung \\
\hline Atomkern & Thermische Neutronen & Neutronenstreuung \\
\hline Innere Elektronen & Röntgenstrahlung & Röntgenstreuung \\
\hline Äußere Elektronen & Lichtwelle & Lichtstreuung \\
\hline $\begin{array}{l}\text { Spezielle } \\
\text { Elektronenanregungszustände }\end{array}$ & Lichtwelle & Fluoreszenz \\
\hline Molekulare Schwingungen & $\begin{array}{l}\text { Lichtwelle } \\
\text { IR-Welle }\end{array}$ & $\begin{array}{l}\text { Ramanstreuung } \\
\text { IR-Absorption }\end{array}$ \\
\hline $\begin{array}{l}\text { Magnetisches Kern-Dipolmoment } \\
\text { Elektrisches Quadrupolmoment }\end{array}$ & $\begin{array}{l}\text { Magnetisches } \\
\text { HF-Feld }\end{array}$ & NMR-Absorption \\
\hline Ungepaartes Elektron & $\begin{array}{l}\text { Magnetisches } \\
\text { SHF-Feld }\end{array}$ & ESR-Absorption \\
\hline $\begin{array}{l}\text { Permanentes elektrisches } \\
\text { Dipolmoment }\end{array}$ & $\begin{array}{l}\text { Elektrisches } \\
\text { Wechselfeld }\end{array}$ & $\begin{array}{l}\text { Dielektrische Disper- } \\
\text { sion/Absorption }\end{array}$ \\
\hline Molekulares Volumen & Ultraschallwelle & Ultraschallabsorption \\
\hline Molekulares Potential & $\begin{array}{l}\text { Bewegungs-- } \\
\text { gleichungen }\end{array}$ & Simulationsergebnisse \\
\hline
\end{tabular}

Tabelle 2.1: Methoden zur Bestimmung der Molekülanordnung und Dynamik [9].

die Enthalpie an die Gleichgewichtslage zwischen verschiedenen Freiheitsgraden des Systems. Dabei sind in Lösungen nicht nur die gelösten Moleküle oder Molekülaggregate selbst, sondern auch deren Solvathüllen zu berücksichtigen. Der Vorteil der Ultraschallspektroskopie liegt darin, dass keine künstlich einzubringenden Labels wie Fluoreszenzmarker, radioaktive Isotope, o.ä. - erforderlich sind. Auf der anderen Seite sind nahezu alle molekularen Prozesse mit einer Volumen- oder Enthalpieänderung verbunden, so dass die Ultraschallspektroskopie ein sehr universelles aber auch unspezifisches Verfahren ist. Man muss eine Reihe von Parametern des zu untersuchenden Systems variieren - dies können die Temperatur, Konzentration, Gegenionen oder auch die Konstitution der beteiligten Moleküle sein - um eine 


\section{Grundlagen der Ultraschallabsorptionsspektroskopie}

Zuordnung und Auswertung der einzelnen beobachteten Prozesse zu molekularen Vorgängen zu ermöglichen.

Die eleganteste Möglichkeit, das molekulare Volumen als Sonde zu nutzen, wäre eine Autokorrelationsanalyse des Rauschsignals, aus dessen zeitlichen quadratischen Mittelwert man das Reaktionsvolumen bestimmen könnte und deren Korrelationszeit Aufschluss über die zeitliche Entwicklung der Reaktion gäbe. Die Realisierung dieser Methode, die im vollständigen thermischen Gleichgewicht arbeiten würde, scheitert bisher an einem zu geringen Signal-Rausch-Verhältnis. In der Praxis werden daher Relaxationsmethoden eingesetzt, die dem System eine kleine Vorzugsrichtung aufprägen und seine Antwort darauf beobachten. Bei den Sprungmethoden erfolgt die Änderung eines äußeren, die Gleichgewichtslage beeinflussenden Parameters innerhalb eines sehr kurzen Zeitraums. Hiebei kann es sich z.B. um einen Drucksprung handeln, der mittels einer platzenden Membran innerhalb von ca. $50 \mu s$ abläuft [41]. Im Falle der Frequenzbereichsspektrometrie wird der äußere Parameter Druck mittels einer Schallwelle harmonisch variiert. Während bei den Sprungmethoden die Anstiegszeit der Druckänderung die geringste auflösbare Relaxationszeit auf Mikrosekunden beschränkt, können mittels spektroskopischer Methoden Zeiten bis hinunter zu 0,1 Nanosekunden erfasst werden. Da die aufgeprägten Störungen stets klein im Verhältnis zu Aktivierungsenthalpien oder zur thermischen Energie des Systems sind, lassen sich die Beobachtungen mit Hilfe der linearen Systemtheorie analysieren. Daher ist die Autokorrelationszeit der Dichtefluktuationen identisch mit der Relaxationszeit bei den Sprungexperimenten oder der Spektrometrie.

Eine weitere messtechnische Herausforderung stellt die Breite der zu Relaxationsphänomenen gehörenden Spektralfunktionen dar. Selbst wenn im Zeitbereich die Relaxation mit einer einzelnen Zeitkonstanten erfolgt, bedeutet dies, dass die Debye-Spektralfunktion, die aus der Laplace-Transformation der Exponentialfunktion hervorgeht, in der korrespondierenden Darstellung der Dämpfung pro Wellenlänge eine Frequenzdekade benötigt, um auf ein Fünftel ihres Scheitelwertes abzufallen (vgl. Abschnitt 2.1.1). Somit besitzt bereits diese schmalste aller RelaxationsSpektralfunktionen im Verhältnis zu Resonanzphänomenen eine große Breite und erfordert das Messen des Absorptionskoeffizienten über einen entsprechend großen Frequenzbereich. Dies ist umsomehr erforderlich, wenn man berücksichtigt, dass eventuell mehrere sich überlagernde Relaxationsprozesse mit unterschiedlichen Zeiten oder gar Prozesse mit kontinuierlichen Relaxationszeitverteilungen auftreten können. Die mit einer solchen Forderung nach einem möglichst großen Frequenzbereich verknüpften Ansprüche an die Messapparaturen möge folgendes Beispiel verdeutlichen. Bei der tiefsten für diese Arbeit genutzten Messfrequenz von ca. $100 \mathrm{kHz}$ beträgt der Dämpfungsexponent von reinem Wasser $\approx 2 \cdot 10^{-4} \mathrm{~m}^{-1}$ und steigt bis auf $\approx 2 \cdot 10^{4} \mathrm{~m}^{-1}$ bei $1 \mathrm{GHz}$ an. Die Strecke, die eine Ultraschallwelle benötigt, um auf die Hälfte ihres Ausgangspegels abzufallen (3 dB-Abfall), ändert sich hierbei von $\approx 1,6 \mathrm{~km}$ auf $\approx 16 \mu \mathrm{m}$. Die Breite des Frequenz- und des damit verknüpften Absorptionsbereiches macht es in der Praxis erforderlich, mehrere Messzellen mit unterschiedlichen Verfahren zur Bestimmung des Dämpfungsexponenten $\alpha$ zu nutzen. Oberhalb Frequenzen von ca. $15 \mathrm{MHz}$ kann der Absorptionskoeffizient direkt, mittels Variation der Dicke der druchstrahlten Flüssigkeitsschicht, mit dem in Abschnitt 3.2 beschriebenen Schwingungspulstransmissionsverfahren bestimmt werden. Hier erfolgt bei Wasser ein $3 \mathrm{~dB}$-Abfall der Leistung auf einer Strecke von $\approx 7 \mathrm{~cm}$. 
Bei kleineren Frequenzen wären die benötigten Messstrecken und damit auch die Volumina der Messlösungen zur sicheren Bestimmung von $\alpha$ unpraktikabel groß, so dass hier das im Abschnitt 3.1 dargestellte Resonatorverfahren genutzt wird.

\subsection{Ultraschalldämpfung}

Mit einer Schallwelle breiten sich zeitliche Schwankungen des Drucks $p$, der Dichte $\rho$, der Temperatur $T$ sowie der Teilchenposition $\vec{x}$ der Materie um ihre Ruhelage räumlich aus. In der Kontinuumsmechanik lässt sich aus der Impulserhaltung und der Massenerhaltung, also aus der Navier-Stokes-Gleichung und der Kontinuitätsgleichung, zusammen mit der Zustandsgleichung des Mediums in akustischer Näherung eine Wellengleichung für den Schalldruck $p$ ableiten. Deren wichtigste Lösungen sind ebene harmonische Wellen. Hier hängen die Schallfeldgrößen nur von einer Raumkoordinate ab und schwanken an einem festen Ort zeitlich, bzw. zu einem festen Zeitpunkt räumlich sinusförmig um ihre Gleichgewichtslage. Für hinreichend kleine Frequenzen $f$ und geringe Wärmeleitfähigkeit $\Lambda$ des Fluids erfolgt kein Wärmeaustausch zwischen verschiedenen Volumenelementen, und die Schallausbreitung ist adiabatisch. Die Schallgeschwindigkeit $c_{s}$ ist dabei über die Newton-LaplaceGleichung mit der adiabatischen Kompressibilität $\kappa_{s}$ des Mediums verbunden:

$$
c_{s}=\sqrt{1 / \kappa_{s} \rho}
$$

Nach [35] birgt die Bestimmung von $\kappa_{s}$ aus der Messung der Schallgeschwindigkeit und der Dichte gegenüber der Berechnung aus thermodynamischen Größen eine geringere Gefahr systematischer Fehler.

Bei einer ebenen Welle werden Flächen senkrecht zur Ausbreitungsrichtung periodisch verschoben. Die auftretenden Verformungen der Volumenelemente lassen sich in eine allseitige Kompression, bzw. Dilatation, und eine Scherung zerlegen. Der damit verbundene irreversible Impulsübertrag zwischen den Fluidschichten wird durch die Volumenviskosität $\eta_{V}$ und die Scherviskosität $\eta_{s}$ beschrieben.

In einem hypothetischen idealen Fluid mit verschwindender Viskosität und Wärmeleitfähigkeit, dessen Zustand durch zwei unabhängige lokale thermodynamische Parameter, z.B. Druck $p(\vec{x})$ und Temperatur $T(\vec{x})$ oder Druck $p(\vec{x})$ und Dichte $\rho(\vec{x})$, vollständig beschrieben wird, erfolgen alle Zustandsänderungen reversibel, und der Schall breitet sich ohne Schwächung aus [40].

In realen Flüssigkeiten bewirkt die irreversible Umwandlung von Schallenergie in Wärme hingegen stets eine Dämpfung der Welle. Experimentelle Beobachtungen bestätigen hierbei die Annahme, dass gleiche Schichtdicken $d x$ des Fluids gleiche Anteile der Schallenergie $E$ dissipieren $d E \propto E d x$. Mit der Proportionalitätskonstante $2 \alpha$ erhält man für eine ebene Welle einen exponentiellen Abfall der Schalldruckamplitude $\hat{p}$ über dem Laufweg $x$.

$$
\hat{p}(x)=\hat{p}_{0} \cdot e^{-\alpha x}
$$

Der Dämpfungsexponent $\alpha$ ist die wichtigste Messgröße der Ultraschallspektroskopie. Sein Frequenzverlauf liefert Informationen über die molekulare Dynamik des untersuchten Fluids. 


\section{Hintergrunddämpfung}

Im Folgenden sollen die verschiedenen Ursachen der Energiedissipation von Flüssigkeiten näher betrachtet werden. Man unterscheidet im Allgemeinen zwischen der Hintergrunddämpfung $\alpha_{b}$ und der Zusatzdämpfung $\alpha_{\text {exc }}$. Unter dem Begriff Hintergrunddämpfung werden die „klassische“ Dämpfung aufgrund der Scherviskosität, sofern diese im betrachteten Frequenzbereich nicht relaxiert, die durch Wärmeleitfähigkeit verursachte Schallschwächung sowie alle Relaxationen oberhalb der maximalen Messfrequenz zusammengefasst.

Nach Bhatia [40] bewirkt die Viskosität zwischen den Flüssigkeitsschichten folgenden, quadratisch mit der Frequenz wachsenden Beitrag zur Absorption.

$$
\alpha_{\eta}=\frac{2 \pi^{2}}{c_{s}^{3} \rho}\left(\frac{4}{3} \eta_{s}+\eta_{v}\right) \cdot f^{2}
$$

mit:

$$
\begin{array}{lll}
\eta_{s} & : & \text { Scherviskosität; } \\
\eta_{v}: & \text { Volumenviskosität; } \\
c_{s} & : & \text { Schallgeschwindigkeit; } \\
\rho & : & \text { Dichte; } \\
f & : & \text { Frequenz der Schallwelle. }
\end{array}
$$

Durch die Zustandsgleichung des Fluids sind die Druckschwankungen der Schallwelle mit lokalen Temperaturschwankungen verknüpft. Der Temperaturgradient treibt aufgrund der nicht verschwindenden Wärmeleitfähigkeit einen Wärmestrom zu den kälteren Volumenelementen und bewirkt damit einen Verlust von Schallenergie. Auch dieser Beitrag zu $\alpha_{b}$ wächst quadratisch mit der Frequenz an [40].

$$
\alpha_{\Lambda}=\frac{2 \pi^{2}}{c_{s}^{3} \rho}\left(\frac{C_{p}}{C_{v}}-1\right) \frac{\Lambda}{C_{p}} \cdot f^{2}
$$

mit:

$$
\begin{array}{lll}
\Lambda & : \text { Wärmeleitfähigkeit; } \\
C_{p} & : \text { spezifische Wärmekapazität bei konstantem Druck; } \\
C_{v} & : \text { spezifische Wärmekapazität bei konstantem Volumen; } \\
c_{s} & : \text { Schallgeschwindigkeit; } \\
f & : \text { Frequenz der Schallwelle. }
\end{array}
$$

Auch der tieffrequente Anstieg der Dämpfung einer Einzelzeitrelaxation $\alpha_{r}$ mit einer Relaxationsrate (weit) oberhalb des Messfrequenzbereichs erfolgt quadratisch mit der Frequenz (vgl. Abschnitt 2.1.1). Zusammenfassend gilt daher für die Hintergrunddämpfung

$$
\alpha_{b}=\alpha_{\eta}+\alpha_{\Lambda}\left(+\alpha_{r}\right)
$$

und man erhält folgende Frequenzabhängigkeit:

$$
\alpha_{b} \propto f^{2} \Longleftrightarrow \frac{\alpha_{b}}{f^{2}}=\text { const. }
$$

Für reines Wasser bei einer Temperatur von $25^{\circ} \mathrm{C}$ und Frequenzen unterhalb $10 \mathrm{GHz} 1$ misst man $\alpha / f^{2}=21,24 \cdot 10^{-15} \mathrm{~s}^{2} / \mathrm{m}$. Bei der Auswertung von Ultraschallspektren

\footnotetext{
${ }^{1}$ Für hohe Frequenzen erfolgt die Schallausbreitung aufgrund der kurzen Wellenlängen, und damit großen Temperaturgradienten, nicht mehr adiabatisch sondern isotherm [40].
} 
wird die Hintergrunddämpfung häufig als sog. $B$-Wert angegeben, der über $B=$ $\alpha_{b} \lambda / f$ berechnet wird. Damit lautet der Hintergrundanteil der Absorption in der Darstellung der Dämpfung längs einer Wellenlänge:

$$
(\alpha \lambda)_{b}=B \cdot f
$$

\section{Relaxationsphänomene}

Untersucht man Wasser mit Zusatz von 2-2-wertigen Elektrolyten, wie z.B. $\mathrm{MgSO}_{4}$, so erhält man eine wesentliche größere Ultraschallabsorption als nach Gleichung (2.5) zu erwarten ist. Die Ursache hierfür ist die Existenz innerer Freiheitsgrade im System, so dass dessen Zustand nicht mehr durch zwei thermodynamische Größen allein beschrieben werden kann, im Beispiel die Assoziation/Dissoziation der Ionen. Diese Freiheitsgrade besitzen thermische Gleichgewichtslagen, die von Parametern wie Druck oder Temperatur abhängig sind. Werden diese durch das Einstrahlen einer Schallwelle periodisch variiert, so wird die jeweilige Gleichgewichtslage entsprechend geändert. Die Einstellung der inneren Freiheitsgrade auf das neue Gleichgewicht erfolgt nicht instantan, sondern mit einer charakteristischen Relaxationszeit $\tau$, die eine Phasenverschiebung zwischen Druck- und Dichteänderungen und damit eine Energiedissipation bewirkt. Sind die Auslenkungen in der Konzentration $c$ aus der Gleichgewichtslage $\bar{c}$ klein, so ist die Reaktionsrate $d c / d t$ proportional zur Abweichung vom Gleichgewicht $(c-\bar{c})$ und man erhält eine exponentielle Annäherung an $\bar{c}$ mit der Relaxationsrate $\tau^{-1}$ als Proportionalitätskonstante:

$$
-\frac{d c}{d t}=\frac{1}{\tau} \cdot(c-\bar{c})
$$

Ändert man die Gleichgewichtslage nicht periodisch sondern sprunghaft, z.B. in einem Drucksprungexperiment, so kann, z.B. über die Leitfähigkeit, die exponentielle Einstellung des Gleichgewichts direkt beobachtet werden. Allerdings können, wie oben erwähnt, auf diese Weise nur Relaxationszeiten gemessen werden, die länger als die Zeit sind, die der Druckabfall benötigt.

\subsubsection{Debye-Spektralfunktion}

Nutzt man ein stationäres Relaxationsverfahren, wie die Einstrahlung einer Schallwelle in die Flüssigkeit, so können wesentlich kürzere Zeiten experimentell erfasst werden. Besitzt die Reaktion ein nicht verschwindendes Reaktionsvolumen, so führt ihr Fortschreiten zu einer Volumenänderung, und während einer Periode wird die folgende Arbeit am System geleistet und in Form von Wärme dissipiert.

$$
W=-\int_{0}^{2 \pi / \omega} p(t) d V(t)
$$

Hier ist $\omega=2 \pi f$ die Kreisfrequenz. Das Arbeitsintegral ist nur dann von Null verschieden, wenn es sowohl ein nichtverschwindendes Reaktionsvolumen als auch eine Phasenverschiebung zwischen $p(t)$ und $V(t)$ gibt. Das Maximum der Absorption liegt bei $\omega \tau=1$. Bei Frequenzen sehr viel kleiner als die Relaxationsrate $1 / \tau$ ist 
das System jederzeit im Gleichgewicht und es tritt keine Phasenverschiebung und damit kein Schallenergieverlust auf. Bei Frequenzen, die sehr viel größer als die Relaxationsrate sind, spürt das System lediglich einen gemittelten Druck und es findet keine Reaktion und damit keine Energiedissipation statt. Somit ist die Zusatzdämpfung $\alpha_{\text {exc }}$ bei gegebenen $\tau$ frequenzabhängig und kann nach [41] wie folgt berechnet werden:

$$
\alpha_{\text {exc }}=\frac{c_{s} \rho}{2 R T} \Gamma\left(\Delta V-\frac{a_{p}}{\rho C_{p}} \Delta H\right)^{2} \cdot \frac{\omega^{2} \tau}{\left(1+\omega^{2} \tau^{2}\right)}
$$

mit:

$$
\begin{array}{lll}
a_{p} & : & \text { thermischer Ausdehnungskoeffizient; } \\
C_{p} & : \text { spezifische Wärmekapazität bei konstantem Druck; } \\
\rho & : \text { Dichte; } \\
c_{s} & : \text { Schallgeschwindigkeit; } \\
\Delta H & : \text { molare Reaktionsenthalpie; } \\
\Delta V & : \text { molares Reaktionsvolumen; } \\
\Gamma & : \text { Gammafaktor. }
\end{array}
$$

Der Gammafaktor folgt aus den Stöchiometriefaktoren $\nu_{i}$ und den Konzentrationen $c\left(A_{i}\right)$ der am Gleichgewicht beteiligten Moleküle:

$$
\frac{1}{\Gamma}=\sum \frac{\nu_{i}^{2}}{c\left(A_{i}\right)}
$$

In der Darstellung der Dämpfung längs einer Wellenlänge erhält man einen DebyeSpektralterm:

$$
\begin{gathered}
(\alpha \lambda)_{\mathrm{exc}}=A_{D} \cdot \frac{\omega \tau}{1+(\omega \tau)^{2}} \\
A_{D}=\frac{\pi c_{s}^{2} \rho}{R T} \Gamma\left(\Delta V-\frac{a_{p}}{\rho C_{p}} \Delta H\right)^{2}
\end{gathered}
$$

Die Amplitude $A_{D}$ gibt die Stärke der Kopplung zwischen Schallwelle und Reaktion wieder und erlaubt somit Rückschlüsse auf die Reaktionsvolumina und die molare Reaktionsenthalpie.

Häufig erhält man im zugänglichen Frequenzbereich mehr als einen Relaxationsprozess mit einer einzelnen Zeitkonstanten, so dass das Spektrum durch eine Summe von Debye-Termen beschrieben werden muss. Bei zusätzlicher Berücksichtigung der Dispersion der Schallgeschwindigkeit (Kramers-Kronig-Relation) erhält man folgende Frequenzdarstellung [42] [41]:

$$
(\alpha \lambda)_{\operatorname{exc}}\left(\frac{c_{s, \infty}}{c_{s}}\right)^{2}=\sum_{i=1}^{N} A_{D_{i}} \frac{\omega \tau_{D_{i}}}{1+\left(\omega \tau_{D_{i}}\right)^{2}}
$$

wobei $A_{D_{i}}$ und $\tau_{D_{i}}$ die Amplitude respektive die Relaxationzeit des $i$-ten Prozesses bezeichnen und $c_{s, \infty}=\lim _{f \rightarrow \infty} c_{s}(f)$.

Schließlich kann aus einer Anpassung mit Debye-Termen der Dispersionsverlauf der Schallgeschwindigkeit berechnet werden, so dass er in die Anpassrechnung einbezogen werden kann:

$$
\left(\frac{c_{s, \infty}}{c_{s}}\right)^{2}=1+\frac{1}{\pi} \sum_{i=1}^{N} A_{D_{i}} \frac{1}{1+\left(\omega \tau_{D_{i}}\right)^{2}}
$$




\section{Messverfahren}

\subsection{Das Resonatorverfahren}

Bei den für diese Arbeit verwendeten Ultraschallresonatoren handelt es sich um zylindrische Hohlraumresonatoren, deren geometrische Abmessungen den jeweiligen Schallwellenlängen angepasst werden. Sende- bzw. Empfangswandler bilden die beiden Stirnflächen des Messvolumens. Als Ultraschallwandler werden EinkristallScheiben aus Quarz verwendet, deren kristallographische x-Achse entlang der Normalen verläuft. Aufgrund des inversen piezoelektrischen Effekts führt der Sender bei einem angelegten elektrischen Wechselfeld Dickenschwingungen der gleichen Frequenz aus und strahlt somit eine Schallwelle in das Messvolumen ab. Das Schallfeld wird vom Empfangswandler detektiert und mittels des piezoelektrischen Effekts wieder in eine elektrische Schwingung transformiert. Zur Ultraschallerzeugung werden piezoelektrische Materialien verwendet, da sie bei den verwendeten Amplituden linear arbeiten. Sie erzeugen eine zur Schallleistung proportionale elektrische Leistung, so dass keine Wandlerkennlinie aufgenommen werden muss. Zur Kontaktierung sind die Quarzscheiben beidseitig zunächst mit einer Chrom- und anschließend mit einer Goldschicht bedampft. Die Bedampfung der Flüssigkeitsseite erstreckt sich bis auf den Mantel der Wandlerscheiben, damit über ein Kupferblech in der Klebung der Kontakt zur Stahlhalterung hergestellt werden kann (vgl. Abbildung 3.3).

In der theoretischen Beschreibung eines idealen Resonators wird die vom Sendewandler abgestrahlte Schallwelle vom Empfangswandler vollständig reflektiert, und im Resonator bildet sich ein stehendes Schallfeld aus; der Schallweg wird in ein kleines Messvolumen gefaltet. Aus der Güte der gemessenen Zellresonanzen kann die Dämpfung und aus deren Frequenzabstand die Schallgeschwindigkeit der zu untersuchenden Flüssigkeit bestimmt werden.

\subsubsection{Der ideale Resonator}

Beim idealen Resonator setzt man lateral unendlich ausgedehnte und schallharte Wandler voraus. Damit können keine Beugungseffekte auftreten, die Schallenergie in den Zellmantel transportieren, und es findet keine Energieabstrahlung auf der Rückseite der Wandler statt. Die Flüssigkeit im Messvolumen ist somit die einzige Ursache der Energiedissipation. Setzt man weiterhin voraus, dass der Sendequarz eine harmonische und ebene Welle abstrahlt, so kann man den Schalldruck unmittelbar am Empfangswandler durch folgende Reihe berechnen, die sämtliche Mehrfachrefle- 
xionen berücksichtigt.

$$
\begin{aligned}
p_{e}= & \hat{p}_{0}(1+r) e^{-\gamma l} e^{i \omega t} \\
& +\hat{p}_{0} r^{2}(1+r) e^{-3 \gamma l} e^{i \omega t} \\
& +\hat{p}_{0} r^{4}(1+r) e^{-5 \gamma l} e^{i \omega t}+\ldots \\
= & \hat{p}_{0}(1+r) e^{-\gamma l} e^{i \omega t} \sum_{n=0}^{\infty}\left(r^{2} e^{-2 \gamma l}\right)^{n} \\
= & \frac{\hat{p}_{0}(1+r) e^{-\gamma l} e^{i \omega t}}{1-r^{2} e^{-2 \gamma l}}
\end{aligned}
$$

mit:

$$
\begin{array}{rll}
p_{e} & : \text { Schalldruck am Empfangswandler; } \\
\hat{p}_{0} & : \text { Schalldruckamplitude der abgestrahlten Welle; } \\
l & : \text { Abstand Sender - Empfänger; } \\
\omega=2 \pi f & : \text { Kreisfrequenz des Sendesignals; } \\
r & : \text { Reflexionsfaktor Flüssigkeit - Wandler; } \\
\gamma=\alpha+i k & : \text { komplexe Ausbreitungskonstante [17]; } \\
\alpha & : \text { Dämpfungsexponent; } \\
k=2 \pi f / c_{s} & : \text { Wellenzahl; } \\
c_{s} & : \text { Schallgeschwindigkeit der Lösung. }
\end{array}
$$

Mit $|r|=1$ für schallharte Reflexion folgt für die Übertragungsfunktion:

$$
T(f) \propto \frac{1}{e^{\gamma l}-e^{-\gamma l}}=\frac{1}{2 \sinh (\gamma l)}
$$

Und mit der Beziehung $\sinh ^{2}(\gamma l)=\sinh ^{2}(\alpha l)+\sin ^{2}(k l)$ erhält man als Betrag dieser Übertragungsfunktion:

$$
|T(f)| \propto \frac{1}{\sqrt{\sinh ^{2}(\alpha l)+\sin ^{2}(k l)}}
$$

Daraus folgt für den betrachteten Fall eines idealen Resonators, dass die Resonanzfrequenzen äquidistant, mit einem Frequenzabstand von $c_{s} / 2 l$, auftreten.

$$
k_{n} l=n \pi \Longleftrightarrow f_{n}=n \cdot \frac{c_{s}}{2 l}, \quad n \in \mathbb{N},
$$

Mit Gleichung (3.4) kann die Schallgeschwindigkeit der Messlösung berechnet werden.

Für kleine Flüssigkeitsdämpfungen $(\alpha l \ll 1)$ kann man Gleichung (3.3) um eine Zellresonanz $f_{n}$ entwickeln. Für $f=f_{n}+\delta f$ gelten die Näherungen: $\sinh (\alpha l) \approx \alpha l$ und $\sin \left(k_{f_{n}+\delta f} l\right)=\sin \left(\frac{2 \pi\left(f_{n}+\delta f\right)}{c_{s}} l\right) \approx \frac{2 \pi \delta f}{c_{s}} l$. Hieraus erhält man für den Frequenzabstand $\delta f_{h}$ einen Abfall der Leistung auf die Hälfte, bzw. des Druckes auf $1 / \sqrt{2}$ seines Scheitelwertes:

$$
\frac{\left|T\left(f_{n}+\delta f_{h}\right)\right|}{\left|T\left(f_{n}\right)\right|}=\frac{1}{\sqrt{2}}=\frac{\sqrt{(\alpha l)^{2}}}{\sqrt{(\alpha l)^{2}+\left(\frac{2 \pi \delta f_{h}}{c_{s}} l\right)^{2}}}
$$


Damit ist eine Verbindung zwischen dem Dämpfungsexponenten $\alpha$, bzw. der Dämpfung längs einer Wellenlänge $(\alpha \lambda)$, und der Halbwertsbreite der Resonanz $\Delta f=2 \delta f_{h}$ hergestellt. Schließlich ergibt sich für geringe Dämpfungen eine Abschätzung von $\alpha$ aus der Halbwertsbreite und der Schallgeschwindigkeit:

$$
\alpha=\frac{\pi}{c_{s}} \Delta f \Longleftrightarrow \alpha \lambda=\pi \frac{\Delta f}{f_{n}}
$$

\subsubsection{Der reale Resonator}

Für einen realen Resonator müssen eine Reihe von weiteren Verlustmechanismen und ihr Einfluss auf die gemessenen Halbwertsbreiten, und damit auf die gesamte Schalldämpfung der Zelle betrachtet werden.

\section{Apparative Verluste, Beugung}

An erster Stelle ist hierbei die endliche laterale Ausdehnung der Messzelle zu nennen. Zu tiefen Frequenzen hin, also bei einem ungünstigen Verhältnis von Wellenlänge und Zellradius, trifft ein zunehmender Anteil der Schallenergie durch die beugungsbedingte Aufweitung des Schallstrahls auf die Mantelfäche des Resonators. Diese Zellberandung besitzt eine materialabhängige Impedanz $Z_{w}$, die einen Teil der auftreffenden Schallenergie in den Außenraum transmittieren lässt. Ferner strahlen die Wandler - insbesondere im Bereich ihrer Resonanzen - einen Teil der Energie rückseitig und in die Halterungen ab. Die einzelnen Verlustmechanismen können für nicht zu große Dämpfungen als unabhängig angenommen werden, da dann die pro Periode reversibel gespeicherte Energie $E_{r}$ groß ist gegen die gesamte pro Periode dissipierte Energie $E_{d}$. Ausgedrückt durch die Güte des Resonators $Q:=2 \pi E_{r} / E_{d}$ bedeutet dies eine Additivität der reziproken Güten:

$$
Q_{\text {gesamt }}^{-1}=Q_{\text {Flüssigkeit }}^{-1}+Q_{\text {apparativ }}^{-1}
$$

Da die Güte, ebenfalls für nicht zu große Dämpfungen, mit der Halbwertsbreite $\Delta f$ der Resonanzkurve über

$$
Q=\frac{f_{n}}{\Delta f}
$$

verknüpft ist, folgt aus der Additivität der reziproken Güten auch eine Additivität der Resonanz-Halbwertsbreiten und schießlich für die Dämpfung längs einer Wellenlänge:

$$
(\alpha \lambda)_{\text {gesamt }}=(\alpha \lambda)_{\text {Flüssigkeit }}+(\alpha \lambda)_{\text {apparativ }}
$$

Nach Labhardt [10] werden die Flüssigkeitsdämpfung, die Beugung und weitere Verlustmechanismen für einen Resonator mit planen Stirnflächen durch folgende Gleichung beschrieben:

$$
\pi \frac{\Delta f}{f}=(\alpha \lambda)_{\mathrm{ges}}=(\alpha \lambda)_{\mathrm{fl}}+\frac{0,147}{\beta_{b}}\left(\frac{c_{s}}{R}\right)^{3} \cdot \frac{1}{f^{3}}+V_{r}
$$


mit:

$$
\begin{array}{lll}
\Delta f & : & \text { gemessene Halbwertsbreite } \\
\alpha & : \text { Dämpfungsexponent der Flüssigkeit; } \\
c_{s} & : \text { Schallgeschwindigkeit der Flüssigkeit; } \\
R & : \text { Radius der Zelle; } \\
\beta_{b} & =\frac{Z_{f l}}{Z_{w}}=\frac{\rho c_{s}}{\rho_{w} c_{w}} \\
V_{r} & : \text { restliche Verlustursachen. }
\end{array}
$$

Der zweite Summand in Gleichung (3.10) beschreibt die Beugungsverluste, alle anderen apparativen Dämpfungsursachen werden in $V_{r}$ subsummiert. Man erkennt einen starken $f^{-3}$ Anstieg der Beugungsverluste zu tiefen Frequenzen, der schließlich die Flüssigkeitsdämpfung vollständig überdeckt. Auf der Seite hoher Frequenzen steigt diese hingegen quadratisch mit der Frequenz an und die Beugung spielt keine Rolle mehr.

Um den Frequenzbereich der Resonatoren zu tiefen Frequenzen zu erweitern, wurden Resonatoren mit konkaven Wandlern konstruiert [11][13][21]. Die Krümmung der Wandler wird durch Schleifen oder durch Anlegen eines leichten Überdrucks in der Messzelle realisiert. Bei sehr dünnen Wandlern (7-MHz-Resonator) reicht bereits der hydrostatische Druck der Flüssigkeit, bzw. eine Verspannung über die Dichtungsringe aus, um die notwendige Verformung zu erzeugen. Die Krümmung eines oder beider Wandler bewirkt eine Fokussierung des Schallstrahls und somit eine Reduzierung der Energie im Bereich des Zellmantels. Auch bei dieser Geometrie erhält man äquidistante Resonanzabstände, die sich wie folgt berechnen lassen [11]:

$$
f_{n}=\left(n+\frac{\arccos (g)}{\pi}\right) \cdot \frac{c_{s}}{2 l}, \quad n \in \mathbb{N}
$$

Für einen bikonkaven Resonator gilt $g=1-l / R_{c}$, für einen plan-konkaven Resonator $g=\sqrt{1-l / R_{c}}$, wobei $R_{c}$ den Krümmungsradius der konkaven Wandler angibt. Die Abbildung 3.1 zeigt den Verlauf der Halbwertsbreiten für einen planplanen Resonator mit 4-MHz-Wandlern und für einen plan-konkaven Resonator mit 1-MHz-Quarzen für eine Wassermessung bei $25^{\circ} \mathrm{C}$. Messlücken ergeben sich sowohl bei den Resonanzen der Dickenschwingung als auch der Scherschwingung der Wandler. Die Scherresonanzen werden aufgrund der elektro-mechanischen Kopplung auch bei x-Schnitt-Quarzen angeregt. Ihre Resonanzfrequenz liegt bei ca. 3/5 der Dickenresonanz [11]. Die Beugungsverluste begrenzen den nutzbaren Messbereich der Zylinderresonatoren zu tiefen Frequenzen. Eine Ausdehnung würde eine Vergrößerung des Wandlerradius bedeuten; die damit verbundene Vergrößerung des Messvolumens ist vielfach aufgrund der zur Verfügung stehenden Messsubstanzmengen nicht praktikabel oder scheitert am Fehlen geeigneter Wandler. Durch Verwendung von Kugelresonatoren lassen sich die Beugungsverluste umgehen, allerdings sind mit diesem Verfahren bisher nur Messungen für Lösungen mit hinreichend hohen Dämpfungen möglich [16].

\section{Apparative Verluste, Referenzmessung}

Die Gesamtheit aller apparativen Verluste lässt sich theoretisch nicht erfassen und ist über längere Zeiträume nicht stabil. Daher kann sie nicht berechnet werden, 


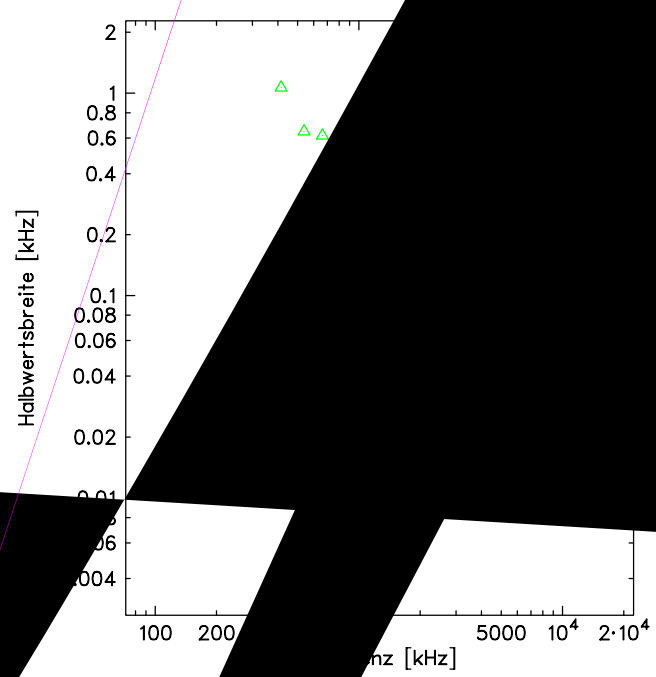


mit:

$$
\begin{array}{lll}
n & : & \text { Nummer der Resonanz; } \\
c_{s} & : \text { Schallgeschwindigkeit der Flüssigkeit; } \\
l & : & \text { Abstand der Wandler; } \\
c_{q} & : \text { Schallgeschwindigkeit in Quarz; } \\
\rho_{q} & : \text { Dichte von Quarz; } \\
f_{q} & : \text { Quarzgrundresonanzfrequenz. }
\end{array}
$$

Somit kann bei bekannter Schallgeschwindigkeit die genaue Zelllänge berechnet werden. Da diese über längere Zeiträume etwas schwanken kann, wird zunächst eine Wassermessung ausgeführt. Anschließend wird anhand der aktuellen Zelllänge die Schallgeschwindigkeit der Messlösung bestimmt.

Um den Einfluss der Wandlerresonanzen auf die auf die Zellresonanzen und damit auf die Dämpfung zu erfassen, kann folgende Funktion $E(f)$ an die Maxima der Hauptmoden angepasst werden.

$$
E(f)=\frac{A \cdot e^{-B f}}{1+\left(d \cdot \tan \left(\frac{\pi}{2}\left(\frac{f}{f_{q n}}+1\right)\right)\right)^{2}}
$$

Hiermit lassen sich die gemessenen Zellresonanzen normieren. $E(f)$ ist die modifizierte Wandlerübertragungsfunktion nach Eggers [15], die um den Faktor $\exp (-B f)$ erweitert wurde, um zusätzlich das elektrische Übersprechen sowie nachfolgende Wandlerresonanzen zu erfassen [27]. Bei hinreichend kleinen Absorptionen und damit schmalen Zellresonanzen ist diese Korrektur nicht erforderlich, da der Einfluss der Quarze über die Resonanzbreite als konstant angesehen werden kann.

\section{Nebenmoden}

Die endlichen Zellradien und zusätzlich die Krümmung der Wandler führen neben den bisher angesprochenen Zellresonanzen, den Hauptmoden, zu weiteren Resonanzmoden. Diese sogenannten Nebenmoden besitzen radiale Knotenflächen und schließen jeweils am hochfrequenten Ende an die Hauptmoden an. Labhardt hat für eine plan-plane Resonatorgeometrie den folgenden Frequenzabstand der Nebenmoden zur Hauptmode berechnet [10]:

$$
f_{n, j}-f_{n}=\frac{1}{8}\left(\frac{c_{s}}{R}\right)^{2} \frac{\left(j-\frac{1}{4}\right)^{2}-\left(\frac{3}{4}\right)^{2}}{f_{n}}
$$

mit:

$$
\begin{array}{lll}
j & : \text { Nummer der Mode }(j=1: \text { Hauptmode }) \\
f_{n} & : \text { Frequenz der n-ten Hauptmode; } \\
c_{s}: & \text { Schallgeschwindigkeit der Flüssigkeit; } \\
R & : \text { Zellradius. }
\end{array}
$$

In Gleichung (3.15) ist entscheidend, dass sich der Resonanzabstand bei zunehmender Frequenz $f_{n}$ verringert, und es zu einer Überlagerung von Haupt- und Nebenmoden kommt (vgl. Abbildung 3.4). Bei plan-konkaver Resonatorgeometrie haben die Nebenmoden zunächst für Wellenlängen sehr viel kleiner als die Wandlerradien 
einen konstanten Abstand zur Hauptmode [11]:

$$
f_{n, j}-f_{n}=j \frac{c_{s}}{\pi l} \arccos \left(\sqrt{1-\frac{l}{R_{c}}}\right)
$$

mit:

$$
R_{c} \quad: \text { Krümmungsradius der konkaven Wandler }
$$

Bei kleinen Frequenzen wird hingegen experimentell ein Verhalten wie beim planplanen Resonator beobachtet [13][12].

\title{
3.1.3 Blockschaltbild der Resonatormessplätze
}
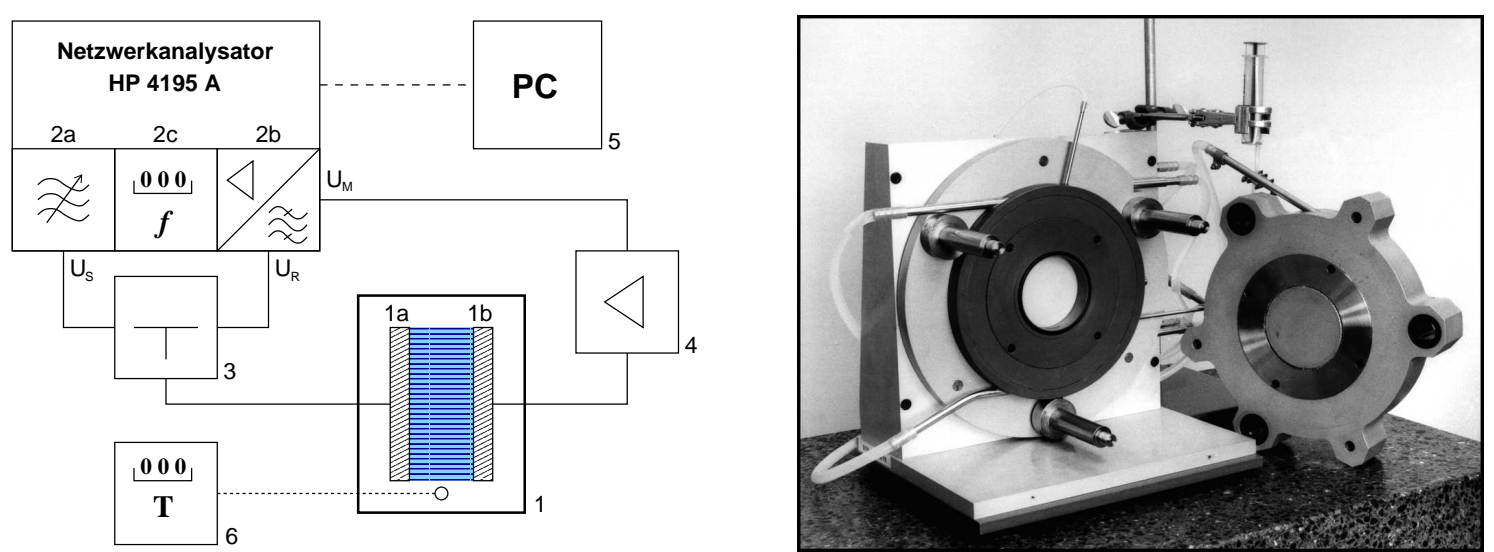

\begin{abstract}
Abbildung 3.2: Links: Blockschaltbild der Resonatormessplätze: (1) Messzelle; (1a) Sendewandler; (1b) Empfangswandler; (2) Netzwerkanalysator (HP 4195A); (2a) Signalgenerator; (2b) Zweikanalempfänger; (2c) Frequenzzähler; (3) Leistungsteiler; (4) $40 \mathrm{~dB}$-Verstärker (10 dB-Schritte) oder FET-Verstärker; (5) Steuerrechner;

(6) Digitalthermometer mit PT-100 Fühler.

Rechts: Foto des offenen plan-konkaven 1-MHz-Resonators.
\end{abstract}

Abbildung 3.2 zeigt ein Schaltbild des Resonatormessplatzes. Zur Signalerzeugung und Analyse der Übertragungsfunktion wird ein Netzwerkanalysator (NWA, HP 4195 A) verwendet. Dieser erzeugt ein kontinuierliches harmonisches Signal $U_{s}$ (2a), das von einem Leistungsteiler (3) in ein Referenzsignal $U_{r}$ und ein Sendesignal getrennt wird. Der Referenzanteil dient dem NWA zur Kontrolle von Signalschwankungen. Das Sendesignal wird an einen der Quarzwandler (1a) angelegt, der die Ultraschallwelle abstrahlt. Der Empfangswandler (1b) detektiert das Schallfeld, und das elektrische Signal wird - bei geringem Pegel zusätzlich verstärkt (4) - dem Messeingang (2b) des NWA als $U_{m}$ zugeführt. Zusätzlich befindet sich bei den hochfrequenten Resonatoren zwischen Empfangswandler und Koaxialleitung eine regelbare aktive Impedanzanpassung. Aus $U_{m}$ und $U_{r}$ wird die Übertragungsfunktion des Resonators berechnet. Die Temperatur wird von einem Thermostaten (Lauda RE 220, RC 20, RM 20) über Thermostatierkanäle im Resonator und eine zusätzliche Hülle vorgegeben und mit einem PT-100-Fühler am inneren Zellmantel überwacht. Die Steuerung des NWA und eine Vorverarbeitung der Messdaten übernimmt ein PC. 


\subsubsection{Resonator-Messzellen}

Insgesamt wurden vier Resonatoren verwendet, wobei jeweils zwei das gewünschte Frequenzintervall überdecken. Im tieffrequenten Bereich zwischen $90 \mathrm{kHz}$ und 3,4 MHz wurden entweder ein plan-konkaver oder ein bikonkaver Resonator mit 1-MHz-Quarzwandlern genutzt. Der Krümmungsradius der geschliffenen Wandler beträgt $2 \mathrm{~m}$. Hochfrequent schlossen ein 4-MHz plan-planer oder ein 7-MHz bikonkaver Resonator an. Die Krümmung des bikonkaven Resonators mit einem Radius von ca. $3 \mathrm{~m}$ wurde durch Quetschen mit den Dichtungsringen erzielt. Tabelle 3.1 enthält die charakteristischen Daten der Resonatoren, und in Abbildung 3.3 ist ein Querschnitt durch den plano-konkaven Resonator gezeigt.

\begin{tabular}{|c|c|ccccccc|c|}
\hline Resonator & Geometrie & $\begin{array}{c}f_{q} \\
{[\mathrm{MHz}]}\end{array}$ & $\begin{array}{c}R_{q} \\
{[\mathrm{~mm}]}\end{array}$ & $\begin{array}{c}R \\
{[\mathrm{~mm}]}\end{array}$ & $\begin{array}{c}l \\
{[\mathrm{~mm}]}\end{array}$ & $\begin{array}{c}f_{g} \\
{[\mathrm{kHz}]}\end{array}$ & $\begin{array}{c}V \\
{\left[\mathrm{~cm}^{3}\right]}\end{array}$ & $\begin{array}{c}R_{c} \\
{[\mathrm{~m}]}\end{array}$ & $\begin{array}{c}\text { Messbereich } \\
{[\mathrm{MHz}]}\end{array}$ \\
\hline $1-\mathrm{MHz}$ & plan-konkav & 1 & 40 & 35 & 19 & 40 & 73 & $2,0^{a}$ & $0,1-3,4$ \\
$1-\mathrm{MHz}$ & bikonkav & 1 & 40 & 35 & 19 & 40 & 73 & $2,0^{a}$ & $0,1-1,7$ \\
$4-\mathrm{MHz}$ & plan-plan & 4 & 10 & 8,4 & 6 & 125 & 1.3 & - & $0,8-15$ \\
$7-\mathrm{MHz}$ & bikonkav & 7 & 10 & 8,4 & 5,5 & 136 & 1.2 & $3,0^{b}$ & $0,8-18$ \\
\hline
\end{tabular}

Tabelle 3.1: Resonatordaten $f_{q}$ : Wandlergrundresonanzfrequenz, $R_{q}$ : Wandlerradius, $R$ : Zellradius, $l$ : Zelllänge, $f_{g}$ : Zellgrundresonanzfrequenz (Füllung mit Wasser bei $\left.25^{\circ} \mathrm{C}\right), V$ : Volumen ohne Befüllschläuche, $R_{c}$ : Wandlerkrümmungsradius, $a$ : geschliffen, $b$ : gequetscht. Angegeben ist der maximale Messbereich des jeweiligen Resonators. In der Praxis ist das nutzbare Frequenzintervall dämpfungs- und schallgeschwindigkeitsabhängig und z.T. deutlich eingeschränkt.

\subsubsection{Messablauf}

Vor jeder Messreihe wird die Paralleljustierung des plan-planen Resonators überprüft und gegebenenfalls korrigiert. Bei den Resonatoren mit konkaver Geometrie ist dieses auf Grund der fokussierenden Wirkung nur selten erforderlich. Die Zellen wurden gründlich gereinigt, mit Methanol gespült und im Stickstoffstrom getrocknet.

$\mathrm{Zu}$ Beginn einer jeden Messserie wird zunächst bei der vorgesehenen Temperatur eine Messung mit gründlich entgastem, destilliertem Wasser ausgeführt. Aus der bekannten Schallgeschwindigkeit des Wassers kann mittels Gleichung (3.13) die Zelllänge berechnet werden. Hierbei ist zu beachten, dass Resonanzen aus dem tieffrequenten Beugungsbereich, der Dicken- und Scherresonanz der Wandler und dem hochfrequenten Anteil mit Nebenmoden unberücksichtigt bleiben, da diese Effekte von Gleichung (3.13) nicht erfasst werden. Außerdem kann mit den bekannten Wassereigenschaften die Funktionstüchtigkeit der Messapparatur überprüft werden.

Anschließend wird die Messung mit der eigentlichen Messsubstanz ausgeführt. Beim Befüllen ist darauf zu achten, dass keine Blasen in den Resonator gelangen, da diese als Streukörper zu merklichen Verlusten führen. Probemessungen bei ausgewählten Resonanzen ermöglichen anhand der Halbwertsbreite eine Abschätzung der Qualität der Befüllung, die bei Bedarf mehrfach wiederholt werden muss. Da das Messvolumen nicht eingesehen werden kann, bleiben als Kriterium für eine gelungene Befüllung nur die Halbwertsbreiten und deren Frequenzverlauf. Anschließend wurde 


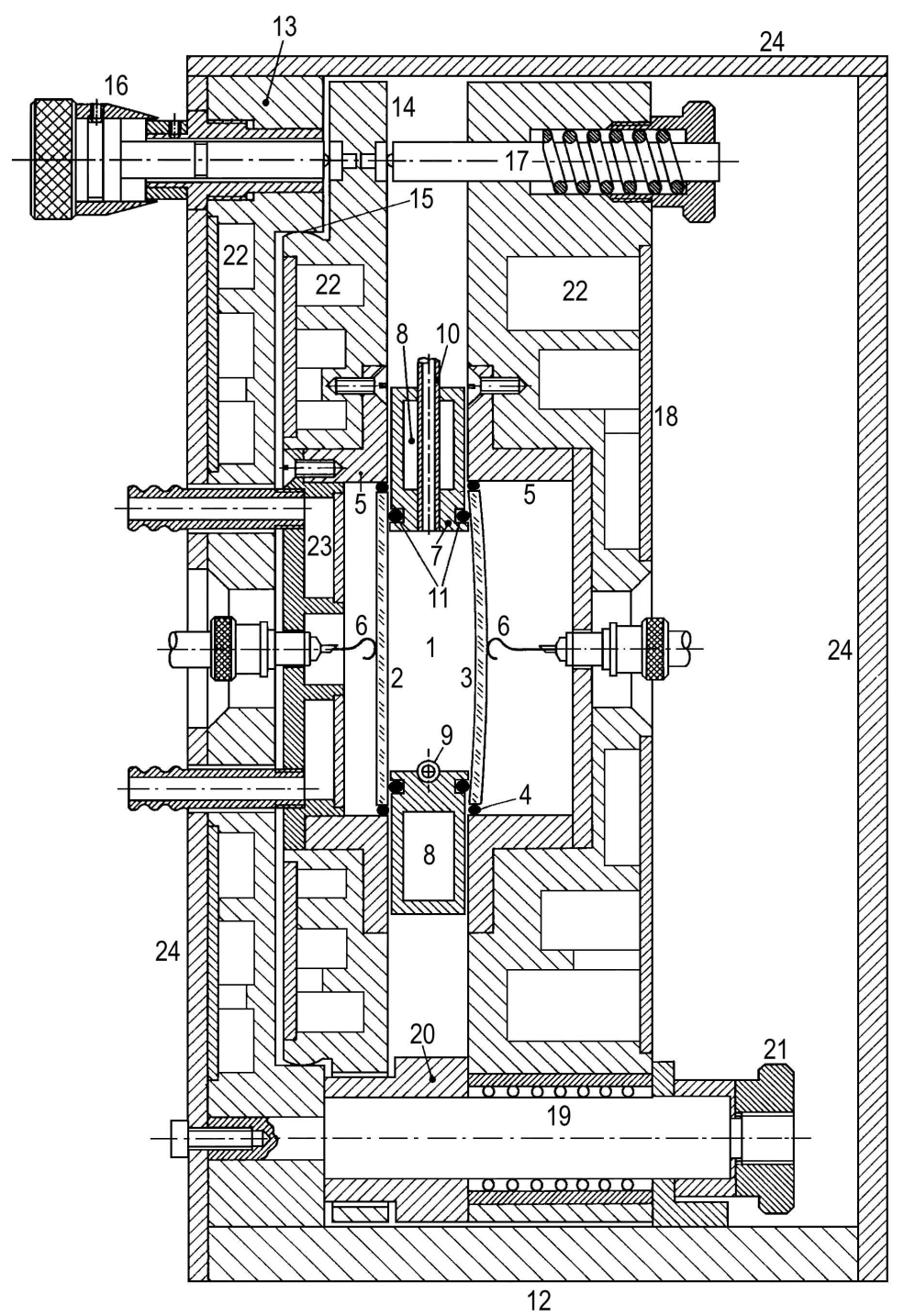

\begin{abstract}
Abbildung 3.3: Querschnitt durch den plan-konkaven Resonator: (1) Messvolumen; (2) planer Wandler; (3) konkaver Wandler; (4) Einklebung mit elektrischer Kontaktierung zur Flüssigkeitsseite; (5) Quarzhalterung (V2A-Stahl); (6) rückwärtige Kontaktierung; (7) Zellberandung; (8), (22), (23) Thermostatierkanäle; (9) Einfüll- und (10) Belüftungskanüle; (11) Dichtungsringe; (12) Grundplatte; (13) Montageplatte der Senderseite; (14) verkippbarer Rahmen zur Paralleljustierung mittels Kugelgelenk (15), Feintriebschraube (16) und Druckfeder (17); (18) Montageplatte der Empfängerseite; (19) Kugelführung; (20) Abstandsstück zur Zelllängenfixierung; (21) Befestigungsschraube; (24) Schutzwände für die Temperaturabschirmung.
\end{abstract}

die Ist-Temperatur so eingeregelt, dass sie maximal 0,03 K von der Soll-Temperatur abwich. Anhand der Mittenfrequenz einer ausgewählten Resonanz wurde die Temperaturdrift innerhalb des Messvolumens beobachtet und bei einer hinreichenden Stabilität mit der Messung begonnen (Drift beim 1-MHz-Resonator $\leq 1 \mathrm{~Hz} / \mathrm{min}$, beim 4 und $7-\mathrm{MHz}-$ Resonator $\leq 10 \mathrm{~Hz} / \mathrm{min})$. Diese ist im Wesentlichen automatisiert und nimmt nacheinander für den vorgegebenen Frequenzbereich die einzelnen 
Zellresonanzen auf. Dabei wird an 100-200 äquidistanten Frequenzpunkten in einem Intervall von ca. drei Halbwertsbreiten die Hauptresonanz nach Betrag und Phase ausgemessen. Aus dem Abstand der Zellresonanzen wird nach Gleichung (3.13) mit der bekannten Zelllänge die Schallgeschwindigkeit der Messlösung bestimmt.

Anschließend wurde eine geeignete Referenzlösung gleicher Schallgeschwindigkeit angesetzt. Im Falle der Kohlenhydratlösungen handelte es sich hierbei fast ausschließlich um wässrige Harnstofflösungen (vgl. Kapitel 5). Nur in Ausnahmefällen wurde auf ein Methanol-Wasser-Gemisch zurückgegriffen. Mit dieser Referenzlösung wurden im Anschluß die apparativen Verluste des Resonators bestimmt. Dabei wurde darauf geachtet, dass sich die Geometrie des Resonators zwischen den Messungen nicht veränderte. Als Kontrolle für einen gelungenen Referenzansatz kann dessen Schallgeschwindigkeit herangezogen werden.

\subsubsection{Anpassrechnung}

Wie Abbildung 3.4 zeigt kann die gemessene Übertragungsfunktion des Resonators i.Allg. nicht durch eine einzelne Lorentz-Funktion beschrieben werden. Daher wird an die Messdaten eine Funktion angepasst, die neben der Hauptmode (n-1) Nebenmoden und das elektrische Übersprechen enthält [12]:

$$
F_{T}(f)=\left(\sum_{j=1}^{n} \frac{A_{j}}{\sinh \left(\gamma_{j} l\right)}+U e^{i \phi_{U}}\right) e^{i \phi_{0}}
$$

mit:

$$
\begin{aligned}
j & : \text { Nummer der Mode (1: Hauptmode); } \\
\gamma_{j} & =\alpha_{j}+i 2 \pi f / c_{s} \text { (Ausbreitungskonstante); } \\
f_{j} & : \text { Resonanzfrequenz der jeweiligen Mode; } \\
\phi_{0} & : \text { globale Phase; } \\
n-1 & : \text { Anzahl der erfassten Nebenmoden; } \\
l & : \text { Zelllänge; } \\
U & : \text { Amplitude des elektrischen Übersprechens; } \\
\phi_{U} & : \text { Phase des elektrischen Übersprechens. }
\end{aligned}
$$

Somit werden für jede aufgenommene Mode drei Parameter und zusätzlich die Amplitude und Phase für das Übersprechen angepasst. Zur Verminderung der Parameterzahl und Vermeidung physikalisch unsinniger Werte können die Parameter jedoch einzeln festgehalten werden. Die vorgegebenen Werte werden für den jeweiligen Resonator aus dessen Geometrie und den Flüssigkeitseigenschaften berechnet.

\subsubsection{Fehler der Resonatormessungen}

Abschließend werden mögliche Fehlerquellen der Resonatormessungen diskutiert und in Tabelle 3.2 eine Fehlerabschätzung für die verschiedenen Messzellen und Frequenzintervalle aufgelistet.

B efullung: Häufigste und schwer abzuschätzende Fehlerquelle bei den Resonatormessungen ist die Befüllung der Messzelle. Luftbläschen im Messvolumen und eine unzureichende Benetzung der Wandleroberflächen führen zu einer Erhöhung der Energiedissipation. Ist die Befüllung sehr schlecht, so kann dies am Minimalwert 


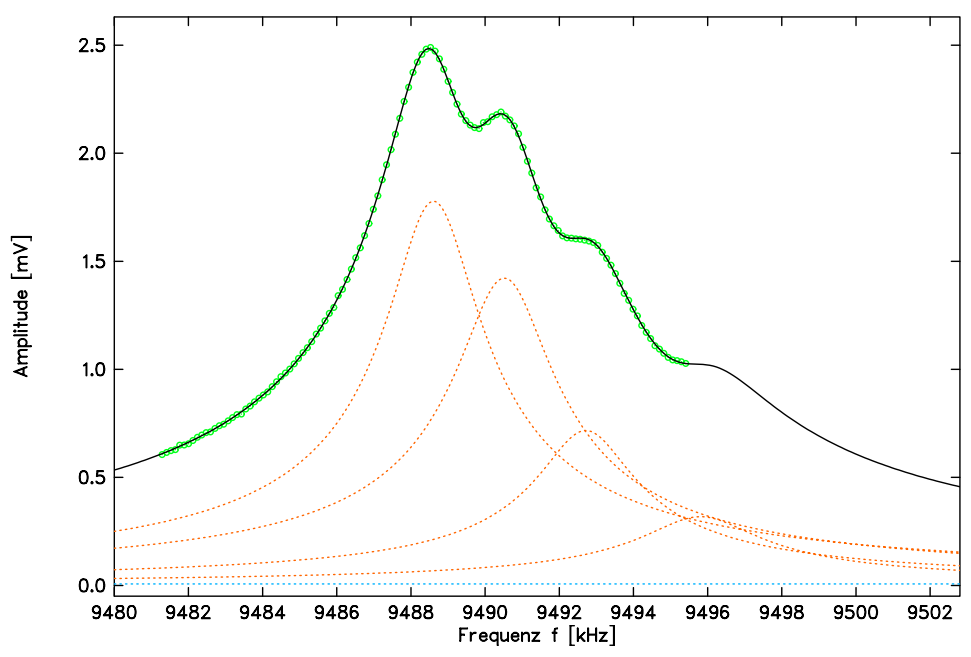

\begin{abstract}
Abbildung 3.4: Betrag der Übertragungsfunktion des 4-MHz-Resonators bei einer Frequenz von 9,488 MHz und Befüllung mit 0,5 molarer wässriger Maltoselösung bei $25^{\circ} \mathrm{C}$. Es wurden insgesamt vier Moden und das elektrische Übersprechen angepasst.
\end{abstract}

der Halbwertsbreite festgestellt werden. Ist der Effekt nur klein, so kann während der Messung nicht entschieden werden, ob die Absorption von der Messflüssigkeit oder auch von Luftbläschen herrührt. In diesem Fall gibt erst der Vergleich mit den Daten von anderen Messzellen Aufschluss. Misslungene Messungen müssen dann bei Bedarf wiederholt werden. Luftblasen stellen gerade bei Kohlenhydratlösungen ein Problem dar, da diese kleine Bläschen stabilisieren, und zusätzlich bei hohen Konzentrationen sehr zäh sind.

Temperaturdrift: Ultraschallresonatoren sind sehr empfindliche Thermometer. Dabei ist für den Messfehler weniger die Änderung der Halbwertsbreite selbst von Bedeutung als vielmehr die Drift der Mittenfrequenz der Resonanz. Um ein Einschwingen zu ermöglichen, werden zum Ausmessen der Zellresonanzen einige Sekunden benötigt. Die Messung der Resonanzkurve erfolgt durch einen Sweep von tiefen zu hohen Frequenzen. Driftet die Mittenfrequenz während dieses Sweeps zu kleineren Frequenzen, so erscheint die Resonanz schmaler, bei einer Drift zu höheren Frequenzen breiter.

Fehler in der R eferenzmessung: Auch für die Referenzmessung gilt oben Gesagtes zur Befüllung der Zelle. Zusätzlich müssen, damit die gleichen apparativen Verluste beschrieben werden, sowohl die Schallgeschwindigkeit wie die Dichte und streng genommen auch die Absorption von Mess- und Referenzlösung exakt übereinstimmen. Wie in Kapitel 5 erläutert, lässt sich mit vertretbarem Aufwand nur einer der Parameter - die Schallgeschwindigkeit - exakt einstellen. Die Dichte weist leichte, die Absorption zum Teil deutliche Abweichungen auf. Dieser Fehler wird jeweils im tieffrequenten Bereich unterhalb des Halbwertsbreitenminimums einer Messzelle (vgl. Abbildung 3.1) dominierend. Daher rührt der größere Fehler für diesen Frequenzbereich in Tabelle 3.2 . 
A npassfehler: Beim Anpassen der Übertragungsfunktion nach Gleichung (3.17) können insbesondere bei hohen Frequenzen systematische Fehler auftreten, da der relative Frequenzabstand der einzelnen Moden immer kleiner und somit eine Unterscheidung zunehmend schwieriger wird. Der verwendete Algorithmus zum Anpassen der Daten optimiert nach mathematischen und nicht nach physikalischen Kriterien.

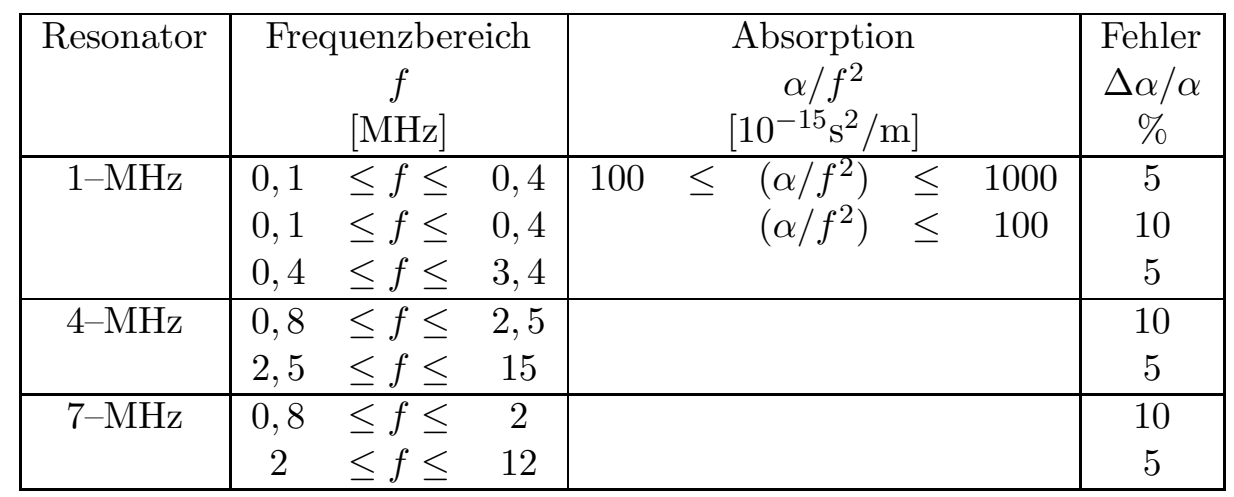

Tabelle 3.2: Fehler der Resonatormessungen. Abschätzung aus Mehrfachmessungen an Lösungen von Sacchariden und Harnstoff, sowie nach [12] [27] [28]. 


\subsection{Das Schwingungspulstransmissionsverfahren}

Geht man vom Resonatorverfahren zu höheren Frequenzen, so ist es ab ca. $10 \mathrm{MHz}$ möglich, den Absorptionskoeffizienten der Lösung direkt über der Länge der Flüssigkeitssäule zu bestimmen, die von der Schallwelle durchlaufen wird. Als einfachste Möglichkeit, die Schallschwächung zu messen, wäre ein Aufbau denkbar, bei dem ein Ultraschallsender und -empfänger sich in einem festen Abstand $d$ gegenüberstehen. Man strahlt eine Schallwelle in die Flüssigkeit ein und misst nach der Strecke $d$ die Amplitude des noch vorhandenen Signals. Ein solcher Aufbau wäre mechanisch einfach zu realisieren, hätte allerdings entscheidende Nachteile. Zum einen müsste das Wandlungsverhältnis von elektrischer in akustische Leistung exakt bekannt sein, zum anderen bräuchte man für einen hinreichend großen Dämpfungsmessbereich verschiedene Zellen mit unterschiedlichen Wandlerabständen. Beide Nachteile kann man mit einem Verfahren, bei dem der Wandlerabstand kontinuierlich verändert wird, vermeiden. Hierbei muss das Wandlungsverhältnis nicht mehr bekannt sein, da es genügt, den Signalpegel bei einem geeignet gewählten Startabstand zu messen und anschließend den Abstand zwischen Sender und Emfänger zu vergrößern. Nach der Definition des Dämpfungsexponenten $p(x)=p(x=0) \cdot e^{-\alpha x}$ ist für die Bestimmung von $\alpha$ nur die relative Änderung von $p(x)$ erforderlich. Geht man davon aus, dass die elektrisch-akustische Wandlung linear erfolgt - diese Voraussetzung ist bei den verwendeten Wandlermaterialien und Signalpegeln gut erfüllt - so müssen die akustischen Größen nicht explizit bekannt sein. Die Wandler der Pulsapparaturen für Ultraschallfrequenzen bestehen aus Quarz- oder Lithiumniobat-Einkristallen, die zur elektrischen Kontaktierung mit dünnen Schichten aus Chrom und Gold bedampft sind. Diese piezoelektrischen Materialien werden mit Hilfe einer gepulsten, harmonischen Hochfrequenzspannung zu Dickenschwingungen gleicher Frequenz angeregt. Die Schallerzeugung bei den Hyperschallapparaturen wird im Abschnitt 3.2.8 dargestellt. Die Messzelle kann bei dieser Methode durch ein Cut-Off-Dämpfungsglied ersetzt werden, um die Übertragungsfunktion der elektronischen Apparatur zu bestimmen (vgl. Abschnitt 3.2.5).

\subsubsection{Blockschaltbild der Pulszellenmessplätze}

Die folgende Abbildung 3.6 zeigt ein Blockschaltbild des Aufbaus der unterschiedlichen für diese Arbeit verwendeten Schwingungspulstransmissions-Messplätze. Nur unmittelbar in der eigentlichen Messzelle wird hierbei mit akustischen Signalen gearbeitet, die Signalerzeugung und Verarbeitung erfolgt auf elektrischem Wege. Die durchgezogenen Linien im Schaltbild geben den Signalweg durch die Messzelle, die gestrichelten Linien denjenigen durch den Referenzzweig wieder. Steuerleitungen zwischen den Messgeräten und dem Steuerrechner werden von gepunkteten Linien repräsentiert. Die Erzeugung der elektrischen Schwingung in Form eines Continuous-Wave-Signals (CW) erfolgt im Frequenzbereich von $3 \mathrm{MHz}$ bis $2 \mathrm{GHz}$ mittels eines Synthetisiersenders (1). Typische Signalamplituden liegen zwischen $-25 \mathrm{dBm}$ und $-5 \mathrm{dBm}$. Um einen Schwingungpuls zu erhalten, wird das CWSignal mit dem Rechteckpuls eines Pulsgenerators an einem PIN-Dioden-Schalter oder einem Dioden-Ring-Mischer gemischt (2) und danach um 40 dB verstärkt (4). Anschließend passiert der Schwingungspuls ein vom Rechner geschaltetes Koaxialrelais (5), mit dem entweder der Mess- oder Referenzzweig gewählt werden können. 

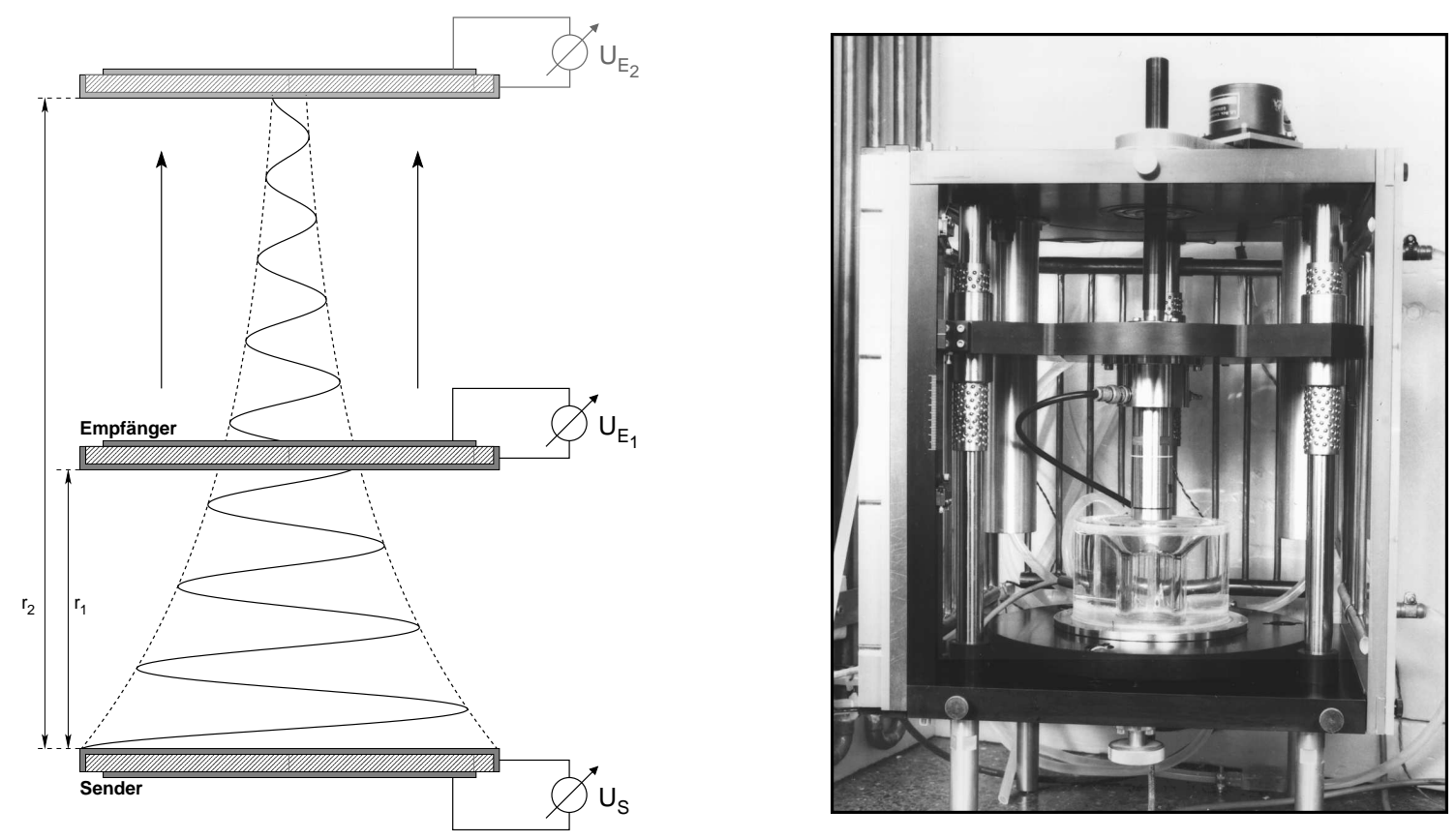

Abbildung 3.5: Rechts: Prinzip der Schwingungspuls-TransmissionsMessung, mit $\mathrm{r}_{i}$ : Abstand der Wandler, $\mathrm{U}_{\mathrm{E}_{\mathrm{i}}}$ : Spannung am Empfangswandler, $\mathrm{U}_{S}$ : Spannung am Sendewandler.

Links: Foto der 6-MHz-Messzelle.

Im Messzweig ermöglicht ein einstellbares Dämpfungsglied (6) die Anpassung der Signalamplituden der beiden Kreise. Im Falle der 10-MHz-Pulszelle und der PZTZelle kommen zusätzlich verlustfreie Impanzanpassungen in Form kurzgeschlossener Stichleitungen variabler Länge zum Einsatz. Diese reduzieren die Reflexionsverluste zwischen den $50 \Omega$-Koaxialleitungen und den Wandlern. Im Folgenden erzeugt der Sendewandler (8a) aus dem elektrischen Signal eine Ultraschallwelle, die in die Messlösung eingestrahlt wird. Nach Durchlaufen einer veränderlichen Flüssigkeitschichtdicke wandelt ein identischer Empfangswandler (8b) das gedämpfte Signal zurück in einen elektrischen Schwingungspuls. Die Variation des Wandlerabstands - und auch der Länge des Rundhohlleites im Cut-Off-Dämpfungsglied - erfolgt durch Schrittmotoren (11) und eine rechnergesteuerte Controller-Karte (21). Aufgrund der sehr kurzen Verfahrwege wird der Abstand bei der PZT-Zelle durch einen Piezotranslator aus Blei-Zirkonat-Titanat (PZT), der mittels einer Hochspannung angesteuert wird, geändert. Das Signal vom Empfangswandler - bzw. aus dem Referenzzweig - gelangt über ein zweites Relais (5) auf einen Überlagerungsempfänger (14 bis 17). Hier wird es mit einem Hilfsoszillator (14) auf eine feste Zwischenfrequenz gemischt und anschließend gefiltert (15), gleichgerichtet (16) und evtl. verstärkt (17). Je nach Frequenzbereich der zur Verfügung stehenden Geräte erfolgt das Heruntermischen bei einigen Messplätzen auch mehrfach. Mit einem Oszilloskop (19) kann das Emfängersignal kontrolliert werden. Ein AnalogDigital-Wandler (18) mit vorgeschalteter Sample-and-Hold-Schaltung ermöglicht das Einlesen der Signalamplitude in den Steuerrechner (25). Dieser steuert zusätzlich die Pulsgeneratoren zur Erzeugung des Senderpulses (3), und der Trigger-Signale für die Sample-and-Hold-Schaltung, A/D-Wandlung und den Oszillographen (20). 
Weiterhin liest er die Vorwärts-/Rückwärts-Zähler (22) der jeweiligen Längentaster aus. Der Abstand wird sowohl zur Bestimmung des Dämpfungsexponenten als auch - bereits während des Messablaufs - zur Berechnung der Verzögerungszeit zwischen Sender- und Emfängertriggerung benötigt. Die Längenmessung erfolgt mit Heidenhain-Metro-Tastern MT60, MT25, MT20 und MT10, die eine Systemgenauigkeit von $0,2 \mu \mathrm{m}$ besitzen. Bei der tieffrequenten Hyperschallzelle wurde bei einem Teil der Messungen ein neuerer Taster des Typs Heidenhain-Certo mit einer Genauigkeit von $0,03 \mu \mathrm{m}$ (lineare Längenfehler-Kompensation in der zugehörigen Messwertanzeige) benutzt. Im Falle der PZT-Messzelle wurde ein genauerer induktiver Längentaster (LVDT Schlumberger) eingesetzt, der eine Reproduzierbarkeit von $5 \mathrm{~nm}$ besitzt [22].

\subsubsection{Grundlagen}

Betrachtet man im ersten Schritt die Verwendung eines reinen CW-Signals, so erhält man beim Transmissions-Verfahren für einen festen Wandlerabstand $x$ die gleichen Bedingungen, die auch in einem Ultraschallresonator vorliegen. Man muss die Mehrfachreflexionen zwischen Flüssigkeit und Wandleroberflächen berücksichtigen und erhält - wieder unter Annahme eines ebenen Wellenfeldes - für den Schalldruck am Empfangswandler folgende Funktion:

$$
p_{e}(x)=\frac{\hat{p}_{0}(1+r) e^{-\gamma x} e^{i \omega t}}{1-r^{2} e^{-2 \gamma x}}
$$

mit:

$$
\begin{array}{rll}
\hat{p}_{0} & : \text { Druckamplitude; } \\
x & : \text { Abstand der Wandler; } \\
\omega=2 \pi f & : \text { Kreisfrequenz; } \\
r & : \text { Reflexionsfaktor (rein reell vorausgesetzt); } \\
\gamma=\alpha+i k & : \text { komplexe Ausbreitungskonstante [17]; } \\
\alpha & : \text { Dämpfungsexponent; } \\
k=2 \pi / \lambda & : \text { Wellenzahl. }
\end{array}
$$

Bei bekannter Senderfrequenz reicht es aus, die folgende Schalldruckamplitude wiederum am Empfangswandler - zu betrachten:

$$
\begin{aligned}
\hat{p}_{e}(x) & \propto\left|\frac{(1+r) e^{-\gamma x} e^{i \omega t}}{1-r^{2} e^{-2 \gamma x}}\right| \\
& =\frac{(1+r) e^{-\alpha x}}{\sqrt{1+r^{4} e^{-4 \alpha x}+2 r^{2} e^{-2 \alpha x} \cos (2 k x)}}
\end{aligned}
$$

Für kleine Wandlerabstände bewirkt die Cosinus-Funktion unter der Wurzel im Nenner eine Welligkeit in der Abstandsabhängigkeit der Schalldruckamplitude mit der halben Wellenlänge in der Flüssigkeit (sogenannte $\lambda / 2$-Welligkeit). Diese klingt mit $e^{-2 \alpha x}$ relativ schnell ab, so dass bei einem Startabstand der Wandler $x_{s}$ größer als $3 / \alpha$ die Reflexionen vernachlässigt werden können, dies gilt dann erst recht für den Summanden $r^{4} e^{-4 \alpha x}$. Damit vereinfacht sich die Gleichung (3.19) zu:

$$
\hat{p}_{e}(x) \propto e^{-\alpha x}
$$




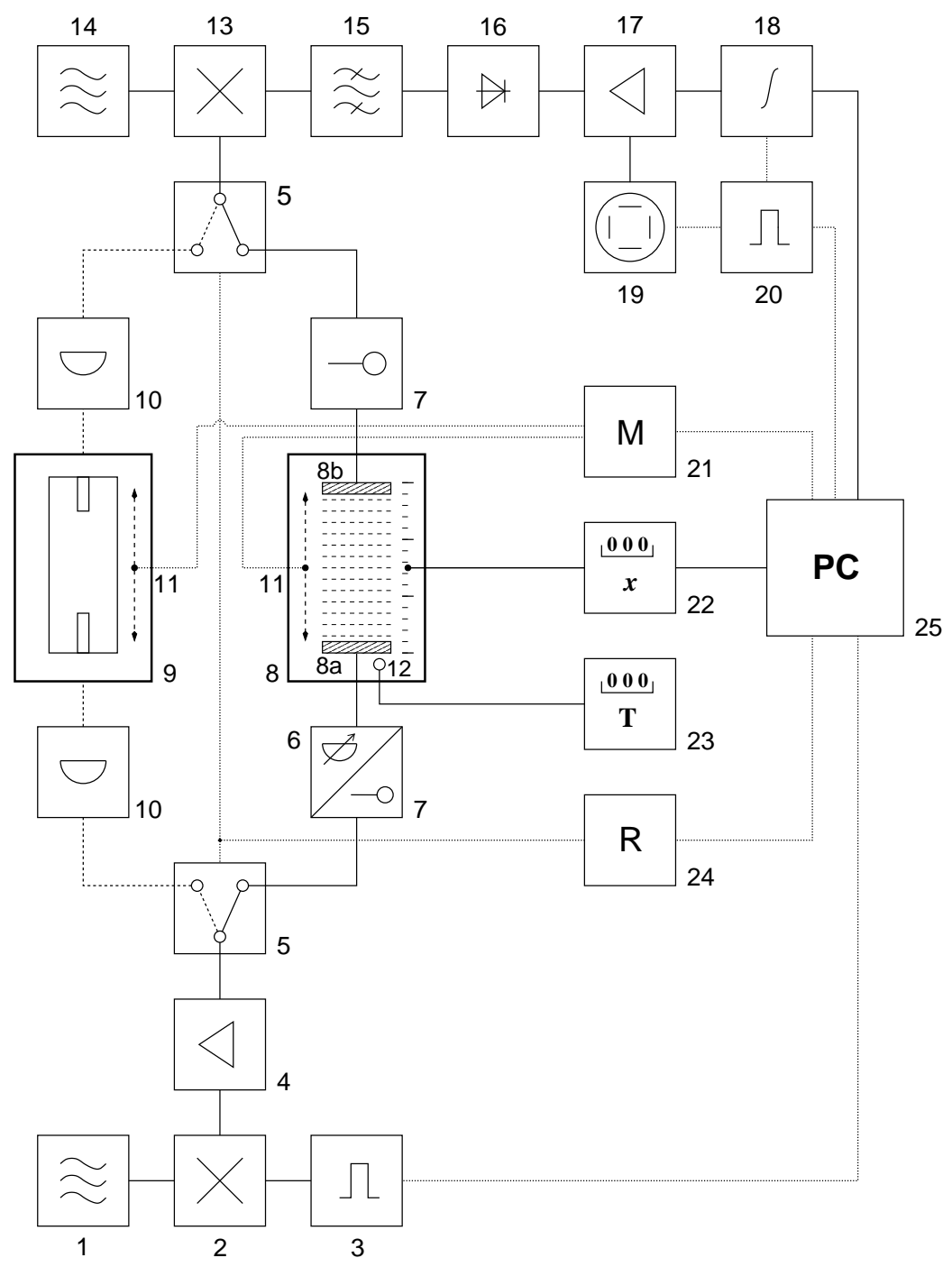

Abbildung 3.6: Blockschaltbild der Pulstransmissionsmessplätze (durchgezogene Linie: Signalleitung durch den Messzweig, gestrichelte Linie: Signalweg durch den Referenzzweig, gepunktete Linien: Steuerleitungen): (1) Synthetisiersender; (2) Pin-Dioden-Schalter oder Dioden-Ringmischer; (3) Pulsgenerator; (4) Verstärker (40 dB); (5) Koaxialrelais; (6) variables Dämpfungsglied zur Amplitudenanpassung; (7) Kurzschlussstichleitungen zur Impedanzanpassung (PZT-Zelle, 10-MHzPulszelle); (8) Messzelle; (8a) Sendewandler; (8b) Empfangswandler; (9) Cut-OffDämpfungsglied; (10) Dämpfungsglieder zum Schutz vor Reflexionen; (11) Schrittmotor oder Piezo-Translator; (12) PT-100 Thermofühler; (13-16) Überlagerungsempfänger; (17) Verstärker; (18) Sample-and-Hold-Schaltung und A/D-Wandler; (19) Oszillograph; (20) Pulsgenerator; (21) Motorsteuerung oder D/A-Wandler mit $1 \mathrm{kV}$-Verstärker; (22) optischer oder induktiver Taster zur Abstandsmessung; (23) Digitalthermometer; (24) Relaistreiberkarte; (25) Steuerrechner.

Schließlich ergibt sich für die Spannung am Empfangswandler:

$$
\hat{U}_{e} \propto \hat{p}_{e}(x) \propto e^{-\alpha x}
$$

Der bereits angesprochene Vorteil des Verfahrens mit variablem Wandlerabstand liegt darin, dass die Proportionalitätskonstante aus Gleichung (3.21) nicht bekannt 
sein muss, da sie lediglich den Achsenabschnitt festlegt, nicht aber die eigentlich interessante Steigung der einfach logarithmischen Auftragung von $\hat{U}_{e}$ gegen $x$ beeinflusst.

\subsubsection{Schwingungspulsverfahren}

Im realen Messbetrieb ist es i.Allg. nicht sinnvoll, einen Startabstand größer als $3 / \alpha$ zu wählen, da hierbei - insbesondere bei schlecht anzuregenden Oberschwingungen der Wandler - zuviel Sendeleistung benötigt wird. Daher werden nicht mehr CWSignale, sondern Schwingungspulse verwendet. Ist die Dauer der Pulse gegenüber der Periodendauer groß genug, so kann, insbesondere unter Berücksichtigung der Wandlerübertragungsfunktion, die auftretende spektrale Verbreiterung der Anregung vernachlässigt werden. Jetzt genügt es, den Startabstand so groß zu wählen, dass sich das Ende des Schwingungspulses am Empfangswandler nicht mit den Echos des Anfangs überlagern kann, also: $x_{\text {Start }}>\tau c_{s} / 2$, mit $c_{s}$ : Schallgeschwindigkeit der Messlösung. Unterschreitet man diesen Startabstand, so erhält man weiterhin eine Welligkeit, die zur Bestimmung der Schallgeschwindigkeit genutzt werden kann (vgl. 3.2.4).

Das Pulsverfahren bietet zusätzlich den Vorteil, dass sich hiermit das akustische Signal zeitlich vom elektrischen Übersprechen trennen lässt. Die Effektivität der Schallwandlung von Quarz bzw. Litiumniobat ist - insbesondere bei Oberschwingung - sehr schlecht. Nur ca. 1/1000 der elektrischen Leistung wird tatsächlich in akustische Leistung umgesetzt. Berücksichtigt man weiterhin die Flüssigkeitsdämpfung, so wird deutlich, dass bereits kleine elektrische Signale, die auf direktem Wege vom Sendekreis in den Empfängerkreis koppeln, die gleiche Größenordnung erreichen können wie das Nutzsignal. Da sich die elektromagnischen Wellen des Übersprechens nahezu mit Lichtgeschwindigkeit, die akustischen hingegen mit Schallgeschwindigkeit ausbreiten, erreichen sie den Empfänger zu unterschiedlichen Zeiten und lassen sich durch passende Triggerung der Sample-and-Hold-Schaltung trennen. Die Übertragungsfunktion der Emfänger begrenzt die Pulsdauer auf Zeiten größer als 1,5 $\mu \mathrm{s}$, so dass bei hochfrequenten Messzellen, mit entsprechend kleinen Startabständen, die Laufzeit des akustischen Signals durch zusätzliche Verzögerungsleitungen erhöht werden muss. Auf diese werden die Wandler zur akustischen Ankopplung mittels Wollfett gebondet.

\subsubsection{Bestimmung der Schallgeschwindigkeit}

Wie oben bereits angesprochen, tritt bei Startabständen $x_{s}$ kleiner als $\tau c_{s} / 2$ und $3 / \alpha$ auch beim Pulsverfahren eine Welligkeit auf. Diese kann nach Gleichung (3.19) dazu genutzt werden, die Schallgeschwindigkeit der Messlösung zu bestimmen. Dabei werden ca. 20-40 Wellenlängen ausgemessen, die Empfängerkennlinie korrigiert (vgl. Abschitt 3.2.5) und die Funktion (3.19) angepasst. Allerdings ist diese Methode der Schallgeschwindigkeitsbestimmung nur bei großen Änderungen des Dämpfungsexponenten genau genug, um den - nach der Kramers-Kronig-Relation an die Absorption gekoppelten - Dispersionsverlauf auszumessen. Während des Messablaufs wird die Schallgeschwindigkeit der Flüssigkeit benötigt, um die Verzögerungszeit des Triggerpulses für die Sample-and-Hold-Schaltung dem sich ändernden Wandlerabstand 
anzupassen. Die Übertragungsfunktionen der Wandler verformen die ursprünglich rechteckige Einhüllende des Schwingungspulses in Richtung einer Dreiecksfunktion, so dass, um systematische Fehler in der Absorption zu vermeiden, die Abtastung stets an der gleichen Stelle des Pulses erfolgen muss.

\subsubsection{Empfängerkennlinie}

Die für die Bestimmung des Dämpfungsexponenten benötigte Messgröße ist die Schalldruckamplitude bzw. die zu ihr proportionale Spannung $\hat{U}_{e}$ am Empfangswandler. Digitalisiert wird das Messsignal allerdings erst nach z.T. mehrfachen Mischen, der Demodulation, Verstärkung etc., die i.a. nicht linear erfolgen. Man benötigt also die Kennlinie des Empfangskreises $\hat{U}_{\text {mess }}=F\left(\hat{U}_{e}\right)$, um aus der gemessenen Spannung die Empfängerspannung berechnen zu können. Die Kennlinie wird mit Hilfe eines Cut-Off-Dämpfungsgliedes im Referenzweig bestimmt. Dieses besteht aus einem Rundhohlleiter, dessen Länge mit Schrittmotoren variiert werden kann. Zum Ein- bzw. Auskoppeln der Welle dienen Koaxialleitungen. Der Hohlleiter wird unterhalb seiner Grenzfrequenz in der radialsymmetrischen $\mathrm{TM}_{01}$-Mode betrieben. Bis auf Nahfeldeinflüsse bei kurzer Hohlleiterlänge erhält man aufgrund der Blinddämpfung einen exponentiellen Abfall der Amplitude über dem Abstand und kann das Signal mit dem zweiten Koaxialleiter aufnehmen. Der Dämpfungsexponent dieser evaneszenten Mode hängt nur von der Hohlleitergeometrie und Anregungsfrequenz ab, und lässt sich wie folgt berechnen [18] [23]:

$$
\alpha_{e}=\frac{\chi_{01}}{r} \cdot \sqrt{1-\left(\frac{2 \pi r}{\chi_{01} \lambda}\right)^{2}}
$$

mit:

$$
\begin{array}{rll}
r & : & \text { Radius des Hohlleiters } \\
\lambda & : \text { Wellenlänge } \\
\chi_{01}=2,405 & : \text { erste Nullstelle der Besselfunktion 1-ter Art }
\end{array}
$$

Das Dämpfungsglied ersetzt zwischen den beiden Koaxialrelais die Messzelle, und gibt dem Emfängerkreis eine definierte Spannung vor, mit der die Kennlinie bestimmt werden kann. Sowohl die Grunddämpfung durch den nicht nutzbaren Nahfeldbereich als auch die Auflösung (Dämpfung pro mm Abstandsänderung) werden durch den den Radius des Hohlleiters vorgegeben. Um für die verschiedenen Frequenzbereiche zwischen $3 \mathrm{MHz}$ und $2 \mathrm{GHz}$ jeweils optimale Anpassung an die Messzelle zu erhalten, wurden drei Dämpfungsglieder mit Radien von $13 \mathrm{~mm}$ (ca. $50 \mathrm{~dB}$ Grunddämpfung), $24 \mathrm{~mm}$ (ca. $25 \mathrm{~dB}$ ) und $56 \mathrm{~mm}$ (ca. $15 \mathrm{~dB}$ ) eingesetzt.

\subsubsection{Beugungskorrektur}

Geht man beim Pulstransmissionsverfahren zu tieferen Frequenzen, so kann die zunehmende Wellenlänge nicht mehr als klein gegen die geometrischen Abmessungen angenommen werden. Das Schallfeld einer Quarzwandlerscheibe, die in eine Stahlhalterung eingeklebt ist, kann näherungsweise als Kolbenstrahlerfeld betrachtet werden, und dieses zeigt nicht zu vernachlässigende Beugungserscheinungen (vgl. Abbildung 3.7). Die Divergenz des Schallstrahls bewirkt, dass mit zunehmendem Wand- 


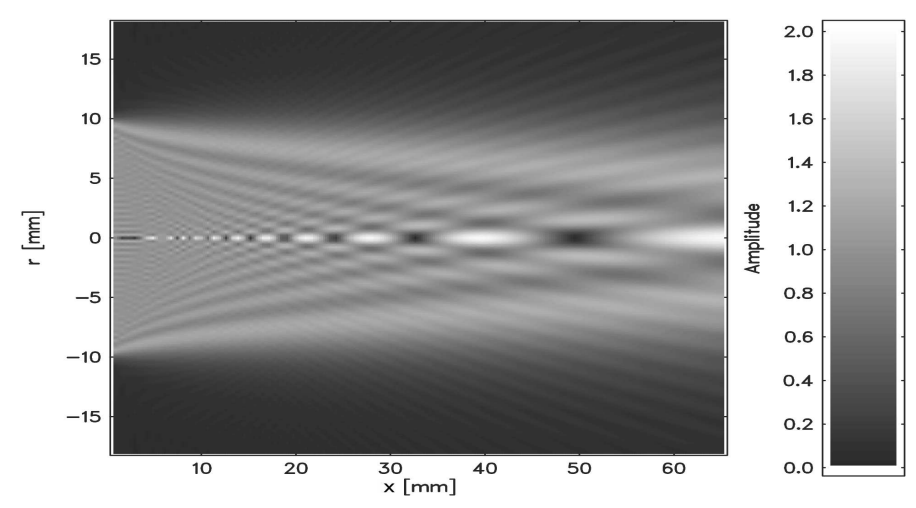

Abbildung 3.7: Berechnetes Schallfeld vor einem idealen Kolbenstrahler mit $10 \mathrm{~mm}$ Radius bei einer Frequenz von $3 \mathrm{MHz}$. Das Medium ist lateral unendlich ausgedehnt und besitzt eine Schallgeschwindigkeit von $1497 \mathrm{~m} / \mathrm{s}$.

lerabstand auch - gänzlich ohne Absorption in der Flüssigkeit - die auf den Empfangswandler treffende Schallleistung abnimmt. Man würde ohne eine Beugungskorrektur mithin eine systematisch zu große Dämpfung messen. Bei den $1-\mathrm{MHz}^{-}$ Pulszellen wird daher bei Frequenzen unterhalb von $50 \mathrm{MHz}$ eine Beugungskorrektur ausgeführt. Mittels numerischer Berechnungen erhält man, unter Berücksichtung der Integration über die gesammte Wandlerfläche, bei gleichen Radien von Sende- und Empfangswandler die unten angebene monotone Schalldruck-AbstandFunktion [19][20]:

mit:

$$
\hat{p}(x)=\hat{p}_{0} \exp \left(-\sqrt{\frac{x}{A \cdot k}}\right)
$$

$$
\begin{array}{lll}
\hat{p}(x) & : \text { Schalldruckamplitude bei Abstand } x \\
\hat{p}_{0} & : \text { Schalldruckamplitude bei Abstand } x=0 \\
x & : \text { Abstand der Wandler; } \\
A & : \text { Fläche der Wandler; } \\
k & : \text { Wellenzahl. }
\end{array}
$$

In der Praxis zeigten sorgfältige Messungen an Flüssigkeiten bekannter Absorption, dass der reale Beugungsverlust durch diese Korrektur überkompensiert wird. Die Ursache hierfür liegt darin, dass das Schwingverhalten des Quarzwandlers von dem eines idealen Kolbenstrahlers abweicht. Die Notwendigkeit einer erneuten Paralleljustierung beim Wechsel der Messfrequenz lässt erkennen, dass Amplitude und Phase über der Wandlerfläche nicht gleich sind. Vermutlich ist die Amplitude der Schwingung zur Mitte der Wandlerplatte größer als im eingeklebten Randbereich. Daher hat Menzel aufgrund der Messungen bei bekannter Absorption folgende empirische Beugungskorrektur vorgeschlagen [20]:

$$
\hat{p}(x)=\hat{p}_{0}\left(\exp \left(-\sqrt{\frac{x}{A k}}\right)\right)^{g(x)}
$$

$g(x) \quad$ : empirisch bestimmter, abstandsabhängiger Gewichtsexponent;

$g(x)=1-x / 100$, für die 1-MHz-Pulszelle (Schwerdtfeger, Tab. 3.3);

$x$ : Wandlerabstand in $\mathrm{mm}$. 
Diese Korrektur hat sich für die 1-MHz-Pulszelle bewährt und wurde für alle Messungen dieser Arbeit verwendet. Weitere Information zur Beugungskorrektur findet man in [21] [12].

\subsubsection{Messablauf}

Im ersten Schritt wurden die Messzellen sorgfältig gereinigt, mit Methanol gespült und im Stickstoffstrom getrocknet. Die Messflüssigkeit wurde entweder unter Vakuum oder im Ultraschallbad entgast und - ebenso wie die Messapparaturen - auf die gewünschte Temperatur vorthermostatiert. Die Lösung wurde vorsichtig, unter Vermeidung von Blasenbildung, eingefüllt und die Temperatur auf eine typische Abweichung von max. 0,03 K auf den Vorgabewert eingeregelt. Außerdem wurde für eine präzise Nachführung des Abtastpulses die Schallgeschwindigkeit der Lösung bestimmt.

Im Anschluss wurde die aktuelle Messfrequenz an Sender und Empfänger eingestellt und feinabgestimmt, die Wandler wurden paralleljustiert und die Signalamplituden in Mess- und Referenzzweig auf den Messbereich der A/D-Wandler von $0,02 \mathrm{~V}$ bis $0,8 \mathrm{~V}$ angepasst, wobei typischerweise ein Startabstand gerade außerhalb der $\lambda / 2$-Welligkeit gewählt wurde. Die Messstrecke wurde, falls die zur Verfügung stehenden Verfahrwege dies zuließen, auf einen Pegelabfall von ca. 15 dB eingestellt.

Entlang dieser Strecke wurde an 400 - 800 Stützstellen der aktuelle Signalpegel und Wandlerabstand gemessen, wobei jeder Spannungswert zur Rauschunterdrückung aus 400 Einzelmessungen gemittel wurde. Um Fehler durch das Spiel zwischen Spindel und Antriebsmutter zu vermeiden, wurde der Abstand kontinuierlich, ohne Zwischenstopps, vergrößert. Die Mittelung über ein Abstandsintervall ist unkritisch, da sie nur zu einer Nullpunktsverschiebung der Längenmessung führt. Zur Bestimmung der Kennlinie wurde im Anschluss an jede Messung auf den Referenzweig umgeschaltet, und der gleiche Dynamikbereich an ca. 60 Stützstellen bei Mittelung über 800 Spannungswerte ausgemessen. Nach Anpassung von Polynomen konnte $\hat{U}_{e}=\mathrm{F}^{-1}\left(\hat{U}_{\text {mess }}\right)$ bestimmt werden. Dann erfolgte eine digitale Filterung und bei Frequenzen unter $50 \mathrm{MHz}$ eine Beugungskorrektur der Rohdaten. Abschließend konnte der Dämpfungsexponent $\alpha$ für die aktuelle Frequenz durch lineare Regresssion an $\left(\log \hat{U}_{e}, x\right)$ bestimmt werden.

\subsubsection{Schwingungspulstransmissions-Messzellen}

Der über das Pulstransmissionverfahren zugängliche Frequenzbereich von ca. $3 \mathrm{MHz}$ bis $5 \mathrm{GHz}$ lässt sich nicht mit einem einzelnen Messplatz nutzen. Die quadratisch ansteigende klassische Ultraschalldämpfung führt zu einer Variation der Messstrecke zwischen $55 \mathrm{~mm}$ und wenigen $\mu \mathrm{m}$. Außerdem sind die Ultraschallwandler und auch die elektronischen Komponenten in ihrem Frequenzbereich begrenzt, so dass zwei Messplätze und insgesamt sieben verschiedene Messzellen benutzt wurden. Diese schließen in ihrem optimalen Frequenzbereich aneinander an, und werden im Folgenden dargestellt. Um eine ausreichende Temperaturstabilität während der Messung zu gewährleisten, sind nicht nur, wie im Folgenden beschrieben, die Messzellen thermostatierbar, sondern zusätzlich befindet sich die gesamte Zelle in einer Themostatierhülle. 


\title{
1-MHz-Pulszellen
}

Im tiefsten mit dem Pulsverfahren genutzten Frequenzbereich von max. $3 \mathrm{MHz}$ bis $100 \mathrm{MHz}$ stehen zwei Messzellen zur Verfügung. Beide sind mit 1-MHz-Quarzscheiben als Ultraschallwandler bestückt. Sie unterscheiden sich in erster Linie in ihrer Ausrichtung auf die chemischen Eigenschaften der untersuchten Lösungen. Im Anschluss wird zunächst die Schwerdtfeger-Zelle beschrieben, deren Querschnittzeichnung in Abbildung 3.8 dargestellt ist. Die verwendeten $1-\mathrm{MHz}-$ Quarzwandler

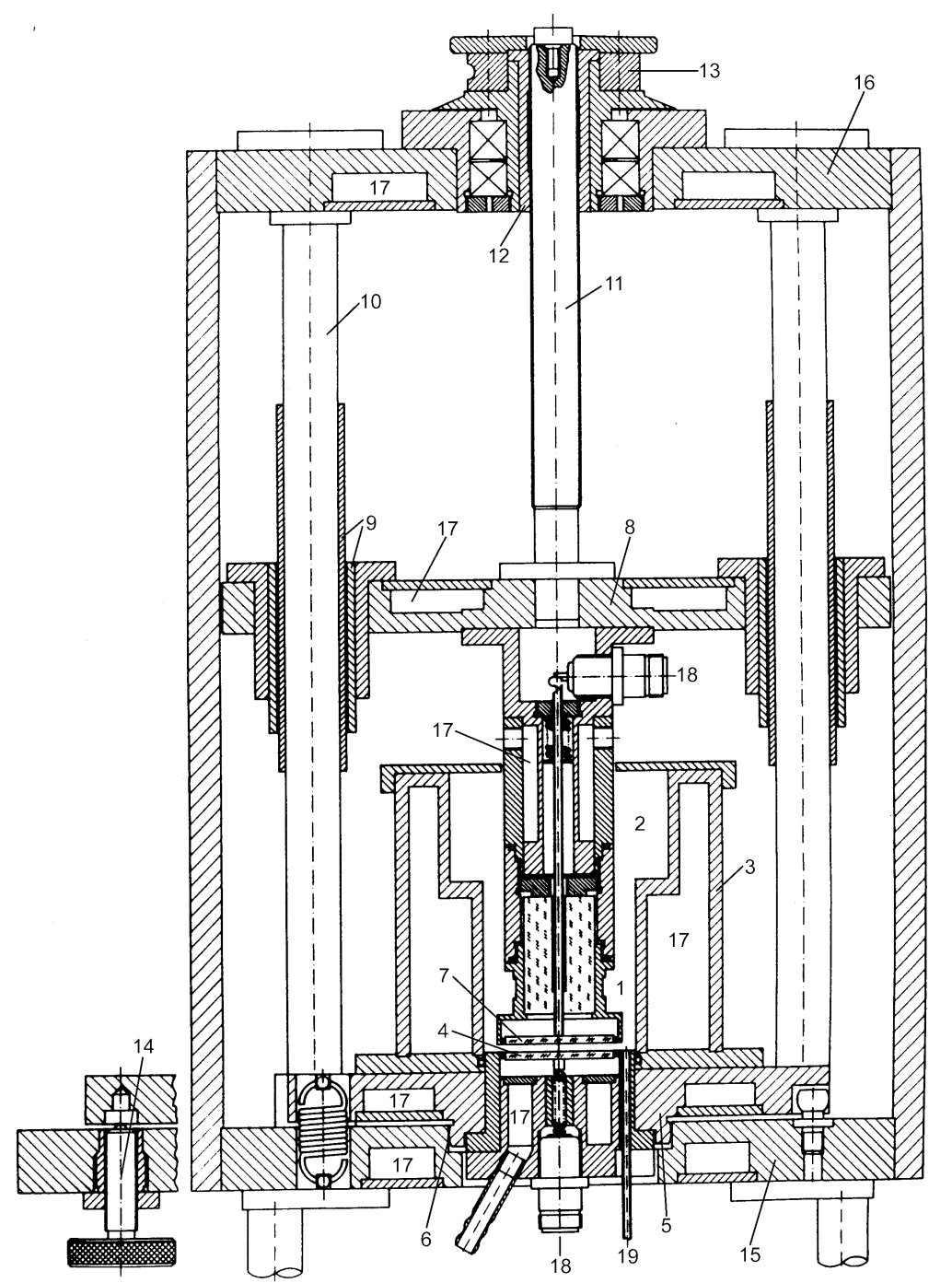

\begin{abstract}
Abbildung 3.8: Querschnitt der 1-MHz-Pulstransmissionszelle (1) Messvolumen mit Vorratsbereich (2); (3) Thermostatiermantel; (4) Senderwandler; (5) kippbare Montageplatte; (6) Kugelgelenk; (7) Empfangswandler; (8) verschiebbare Montageplatte; (9) Kugelführung; (10) Führungssäule; (11) Spindel; (12) Mutter; (13) Riemenscheibe für den Antrieb von (11); (14) Schrauben zur Paralleljustierung; (15) Grundplatte; (16) Deckenplatte; (17) Thermostatierkanäle; (18) N-Norm-Anschlüsse; (19) Ablaufkanüle.
\end{abstract}

besitzen eine ausreichende mechanische Stabilität, ca. 2,8 mm Stärke bei $20 \mathrm{~mm}$ Radius, so dass sie direkt mit der Messlösung in Kontakt gelangen können. Am Rand 
sind beide Quarze mit Silicon (Wacker E42) in Halterungen aus V2A-Stahl geklebt. Die chemische Beständigkeit der Klebung, der Goldbedampfung der Wandler, des Stahls sowie der gläsernen Thermostatierhülle sind für die verwendeten Kohlenhydratlösungen ausreichend.

Die Halterung des Senderquarzes ist auf eine Montageplatte (5) geschraubt, deren Ausrichtung zur Paralleljustierung durch drei Mikrometerschrauben verändert werden kann. Die entsprechende Montageplatte (8) des Empfangswandlers kann von einem Schrittmotor entlang der Wandlernormalen verfahren werden. Drei Präzisionskugelführungen $(9,10)$ verhindern hierbei ein Verkippen gegenüber dem Sendewandler. Der Antrieb erfolgt durch eine Mutter (13), die eine fest an der Empfangswandlerhalterung montierte Spindel (11) bewegt. Somit ist auch sichergestellt, das die Abstandsmessung am oberen Spindelende direkt auf der Wandlermittenachse erfolgt. Die Messflüssigkeit (1) befindet sich in einem transparenten Thermostatiermantel (3), der auf der Außenseite aus Plexiglas, auf der Innenseite jedoch aus Glas besteht, da Plexiglas nicht die nötige chemische Resistenz besitzt. Somit können Luftblasen und evtl. optische Veränderungen der Messlösung direkt erkannt werden. Zur Reduktion der Verdunstung und den damit verbundenen Konzentrationsänderungen wird der Mantel durch einen Deckel aus Teflon verschlossen. Um die notwendige Themperaturkonstanz sicherstellen zu können, sind zusätzlich die Empfangswandlerhalterung, beide Montageplatten, die Boden- und Deckenplatte sowie zwei Seitenwände mit Thermostatierkanälen (17) versehen.

Als Messfreqenzen stehen die elektrisch anregbaren ungeraden Vielfachen der Quarzgrundresonanz zur Verfügung. Die Grundresonanz des Quarzes liefert, wie auch bei allen anderen Pulszellen, keine verwertbaren Messdaten, so dass der Messbereich bei $3 \mathrm{MHz}$ beginnt. Da der maximale Messweg bei dieser Apparatur ca. $55 \mathrm{~mm}$ beträgt, wird ein hinreichender Abfall der Signalamplitude (ca. $3 \mathrm{~dB}$ ) nur bei genügend hohen Flüssigkeitsdämpfungen erreicht. Für Wasser $\left(\alpha / f^{2}\right)=21,24 \cdot 10^{-15} \mathrm{~s}^{2} / \mathrm{m}$ geschieht dies oberhalb von ca. $17 \mathrm{MHz}$. Die obere Frequenzgrenze wird durch die zunehmend schlechtere Anregbarkeit der Wandleroberschwingungen und dem damit verbundenen ungünstigen Signal-Rausch- und Signal-Übersprech-Verhältnis bestimmt. Sie liegt im Bereich von 80 bis $120 \mathrm{MHz}$.

Die Gailus-Zelle ist im Gegensatz zur Schwerdtfeger-Zelle auf bessere chemische Resistenz ausgelegt. Der Thermostatiermantel besteht vollständig aus Edelstahl und ist daher nicht transparent, so dass Luftblasen anhand des Messsignals erkannt werden müssen. Vor den Wandlern wurden Verzögerungsleitungen aus Quarz eingebaut, die nicht geklebt, sondern mit teflonummantelten O-Ringen festgequetscht werden. Auch auf eine geklebte Ablassöffnung wurde verzichtet. Der nutzbare Frequenzbereich entspricht dem der Schwerdtfeger-Zelle, das höhere Flüssigkeitsvolumen (vgl. Tabelle 3.3) fällt bei den meisten Kohlenhydratlösungen nicht ins Gewicht und die Messfehler weichen ebenfalls nicht wesentlich voneinander ab. Daher wurden für diese Arbeit beide Messzellen verwendet.

\section{6-MHz-Pulszelle, 10-MHz-Pulszelle}

Der Aufbau der beiden höherfrequenten Messzellen entspricht prinzipiell dem der 1MHz-Zellen. Unterschiede bestehen bei den Ultraschallwandlern. Während bei der 6-MHz-Zelle weiterhin - entsprechend dünnere - Quarzwandler genutzt werden, sind in der $10-\mathrm{MHz}-$ Zelle Lithiumniobat-Scheiben im Einsatz. Diese Wandler sind 
sehr dünn und das Material spröde, so dass die Verzögerungsleitungen aus Quarz auch als mechanischer Schutz fungieren. Der nutzbare Frequenzbereich beginnt auch hier bei der dritten Oberschwingung und endet bei ca. $500 \mathrm{MHz}$ in erster Linie aufgrund des ungünstigen Signal-Übersprech-Verhältnisses. Die 6-MHz-Zelle wurde im Rahmen dieser Arbeit nur in Ausnahmefällen genutzt, da ihr Frequenzbereich bereits durch die $1-\mathrm{MHz}-$ und $10-\mathrm{MHz}-$ Zellen vollständigt überdeckt wird.

\section{Tieffrequente Hyperschallzelle}

$\mathrm{Zu}$ hohen Frequenzen schließt die tieffrequente Hyperschallzelle an die $10-\mathrm{MHz}^{-}$ Apparatur an. Da hier keine in Resonanz betriebenen Dickenschwinger, sondern die Oberflächenanregung nach Bömmel und Dransfeld [29] zur Schallerzeugung genutzt werden, kann sie ihren Frequenzbereich von $550 \mathrm{MHz}$ bis $2 \mathrm{GHz}$ kontinuierlich überdecken. Als Wandler und gleichzeitig Verzögerungsleitungen dienen LithiumniobatStäbe von $10 \mathrm{~mm}$ Länge, bei einem Durchmesser von $3 \mathrm{~mm}$. Diese Stäbe sind mit Bienenwachs in Stahlhalterungen geklebt und ragen auf der flüssigkeitsabgewandten Seite ca. 1-2 mm in einen Koaxialresonator. Mittels einer Messingspitze, die mittig auf dem Wandler aufsetzt, und der Stahlhalterung wird ein inhomogenes elektrisches Feld erzeugt, das das piezoelektrische Wandlermaterial zu mechanischen Schwingungen anregt. Die mittels Servomotoren abstimmbaren Koaxialresonatoren realisieren für die jeweilige Messfrequenz eine maximale Feldstärke am Wandler. Die Abstandsänderung wird hier durch Verfahren des unteren (Sende-) Wandlers erreicht. Dabei muss der Koaxialresonator mitbewegt werden, ohne dass sich seine Abstimmung verändert. Dies würde zu einer Änderung der Feldstärke und damit der akustischen Sendeleistung führen, die die Änderung des Signalpegels am Empfänger aufgrund der Absorption verfälscht. Da die Koaxialresonatoren unmittelbar in der Wandlermittenachse positioniert werden müssen, kann bei dieser Apparatur die Abstandsmessung nicht axial erfolgen. Der Längentaster ist achsparallel angebracht, so dass Fehler durch ein Verkippen der fahrbaren Montageplatte nicht auszuschließen sind. Da der verwendete Empfänger fest auf 998,8 MHz eingestellt ist, wird das Messsignal an einer nichtlinearen Kennlinie mittels eines Hilfsoszillators auf diese Frequenz gemischt. Die obere Frequenzgrenze von $2 \mathrm{GHz}$ ergibt sich nicht aus den Eigenschaften der Messzelle selbst, sondern wird durch den zur Verfügung stehenden Synthetisiersender festgelegt. Zu tiefen Frequenzen wird der Arbeitsbereich durch die Baulänge der Koaxialresonatoren begrenzt. Zur Bestimmung des Dämpfungsexponenten werden drei Funktionen herangezogen. Bei nicht zu großer Flüssigkeitsdämpfung und vernachlässigbarem Übersprechen erfolgt wie bei der 1- und 10-MHz-Zelle eine lineare Regression an $\left(\log \left(\hat{U}_{e}\right), x\right)$. Bei zunehmender Flüssigkeitsdämpfung wird der Startabstand so klein gewählt, dass ca. $1 / 3$ der Messstrecke in der $\lambda / 2$-Welligkeit liegt (siehe Abb. 3.9). In diesem Fall wird zunächst die Übertragungsfunktion nach Gleichung (3.25) ohne Übersprechen angepasst.

$$
\hat{p}_{e} \propto\left|A_{0} \frac{(1+r) e^{-\gamma\left(x-x_{0}\right)}}{1-r^{2} e^{-2 \gamma\left(x-x_{0}\right)}}+A_{e l} e^{i \phi_{e l}}\right|
$$

Hierbei bezeichnet $x_{0}$ eine Nullpunktsverschiebung der Abstandmessung, $A_{e l}$ und $\phi_{e l}$ die Amplitude und relative Phase des elektischen Übersprechens. Bei dieser Anpassung erhält man zusätzlich die Schallgeschwindigkeit bei der gegebenen Frequenz. 
Falls sich aufgrund des Übersprechens eine hinreichend modulierte $\lambda$-Welligkeit in den Rohdaten zeigt, wird auch diese in den Anpassrechnungen berücksichtigt.

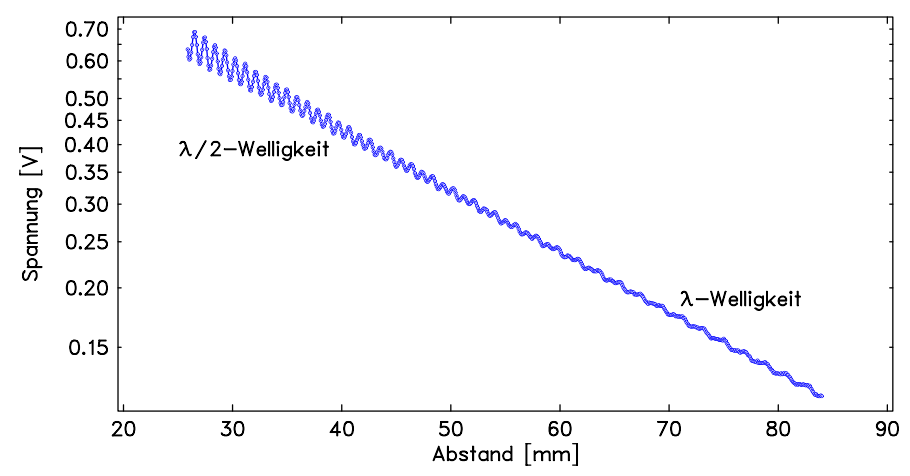

Abbildung 3.9: Beispiel für Welligkeiten in der Übertragungsfunktion. Messung von 0,5 molarer wässriger Melibioselösung bei $10^{\circ} \mathrm{C}$ und $790 \mathrm{MHz}$ mit der tieffrequenten Hyperschallzelle.

\section{PZT-Zelle}

Für das Frequenzintervall von 0,6-4,6 GHz wird zur Schallerzeugung wieder das Prinzip eines piezoelektrischen Dickenschwingers genutzt. Die hierfür benötigten Wandler mit einer Grundresonanz im Bereich von $1 \mathrm{GHz}$ sind so dünn, dass sie nicht mehr geschnitten bzw. geschliffen werden können. Als Material wird hier Zinkoxid verwendet, dass auf eine Verzögerungsleitung aus Saphir gesputtert wurde. Zur Kontaktierung wurde diese zuvor mit einer Chrom- Goldschicht-Kombination versehen. Allerdings läßt sich die nur ca. $1 \mu \mathrm{m}$ starke ZnO-Schicht nicht mehr als unabhängig vom Träger betrachten. Das System aus Wandler, Gold--, Chromschicht und Saphir besitzt breite, wenig ausgeprägte Resonanzen mit einer Grundfrequenz von ca. 1,3 GHz. Dies ermöglicht, bei einer ausreichenden Flüssigkeitsdämpfung Messungen von $600 \mathrm{MHz}$ bis $2 \mathrm{GHz}$ mit einer nahezu beliebigen Wahl der Senderfrequenz. Für den Messbereich bis 2 GHz wurde ein Synthetisiersender genutzt (HP 8675 B).

Die für diesen Frequenzbereich nötige Präzision der Abstandsänderung wird nicht mehr durch einen Spindelantrieb, sondern durch einen Piezotranslator aus BleiZirkonat-Titanat (PZT) erzielt. Dieser besitzt zusammen mit der Längenmessung eine Reproduzierbarkeit von ungefähr $5 \mathrm{~nm}$ [22] und einen maximalen Verstellweg von $48 \mu \mathrm{m}$. Die Ansteuerung des Translators erfolgt über einen Digital-AnalogWandler mit nachgeschaltetem Hochspannungsverstärker. Da die Abstandsauflösung der Heidenhain-Metro-Taster bei diesen kleinen Abstandsvariationen nicht mehr ausreicht, erfolgt die Längenmessung mit einem induktiven Taster (LVDT Schlumberger). Dieser muss jedoch vor jeder Messung mit Hilfe eines Heidenhain-Tasters kalibriert werden.

Bei dieser Zelle ist es nicht mehr möglich, außerhalb der Welligkeiten zu messen. Daher wird auch hier die Übertragungsfunktion nach Gleichung (3.25) an die kennlinienkorrigierten Messdaten angepasst, wobei ebenfalls, je nach Modulation des Signals, das Übersprechen berücksichtigt wird. 


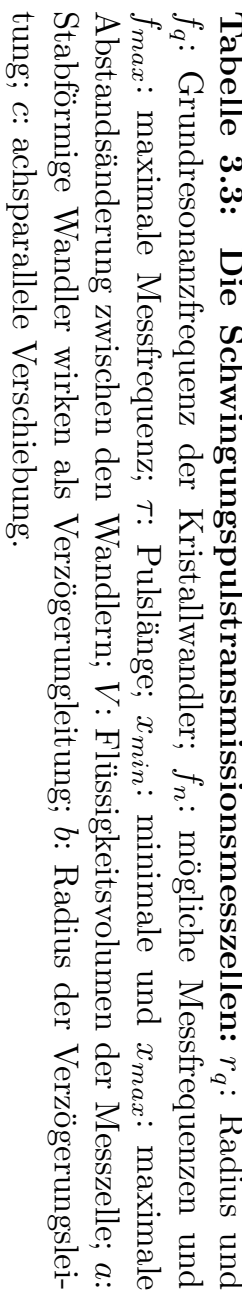

\begin{tabular}{|c|c|c|c|c|c|c|}
\hline Zelle & $\begin{array}{c}\text { 1-MHz- } \\
\text { Pulszelle } \\
\text { (Gailus) }\end{array}$ & $\begin{array}{c}\text { 1-MHz- } \\
\text { Pulszelle } \\
\text { (Schwerdtfeger) }\end{array}$ & $\begin{array}{l}6-\mathrm{MHz}- \\
\text { Pulszelle } \\
\text { (Praktikum) }\end{array}$ & $\begin{array}{l}\text { 10-MHz-- } \\
\text { Pulszelle }\end{array}$ & $\begin{array}{c}\text { Hyperschall- } \\
\text { zelle }\end{array}$ & $\begin{array}{l}\text { PZT- } \\
\text { Zelle }\end{array}$ \\
\hline Wandlermaterial & Quarz & Quarz & Quarz & $\mathrm{LiNbO}_{3}$ & $\overline{\mathrm{LiNbO}_{3}}$ & $\mathrm{ZnO}$ \\
\hline Verzögerungsl. & ja & nein & ja & ja & $\mathrm{ja}^{a}$ & ja \\
\hline$r_{q}[\mathrm{~mm}]$ & $17,5^{b}$ & 20 & $7,5^{b}$ & 6 & 1,5 & $3,5^{b}$ \\
\hline$f_{q}[\mathrm{MHz}]$ & 1,0 & 1,05 & 6 & 10,8 & breitbandig & $\approx 1300$ \\
\hline$f_{n}$ & $(2 n+1) f_{q}$ & $(2 n+1) f_{q}$ & $(2 n+1) f_{q}$ & $(2 n+1) f_{q}$ & $0,5-2,0 \mathrm{GHz}$ & $0,6-2,0$ \\
\hline$f_{\max }[\mathrm{MHz}]$ & 100 & 100 & 174 & 530 & 2000 & $\begin{array}{c}3,7-4,6 \mathrm{GHz} \\
4600\end{array}$ \\
\hline$\tau[\mu \mathrm{s}]$ & $4-8$ & $4-8$ & $3-6$ & $2-4$ & 1,5 & 1,5 \\
\hline Taster & $\begin{array}{l}\text { optisch, } \\
\text { MT60 }\end{array}$ & $\begin{array}{l}\text { optisch, } \\
\text { MT60 }\end{array}$ & $\begin{array}{l}\text { optisch, } \\
\text { LS103 }\end{array}$ & $\begin{array}{l}\text { optisch, } \\
\text { MT20, } \\
\text { MT25 }\end{array}$ & $\begin{array}{l}\text { optisch, } \\
\text { MT10, } \\
\text { CT2505 }\end{array}$ & $\begin{array}{l}\text { induktiv, } \\
\text { LVDT }\end{array}$ \\
\hline Tasterposition & axial & axial & $20 \mathrm{~cm}^{c}$ & axial & $7 \mathrm{~cm}^{c}$ & axial \\
\hline$x_{\min }[\mathrm{nm}]$ & 125 & 125 & 125 & 125 & 0,5 & 8 \\
\hline$x_{\max }[\mathrm{mm}]$ & 55 & 55 & 25 & 25 & 1 & 0,04 \\
\hline$V[\mathrm{ml}]$ & $\approx 200$ & $\approx 130$ & $\approx 25$ & $\approx 10$ & $\approx 3$ & $\approx 0.5$ \\
\hline Literatur & {$[28]$} & {$[25]$} & {$[30]$} & [24] & {$[20,26]$} & {$[22,26]$} \\
\hline
\end{tabular}

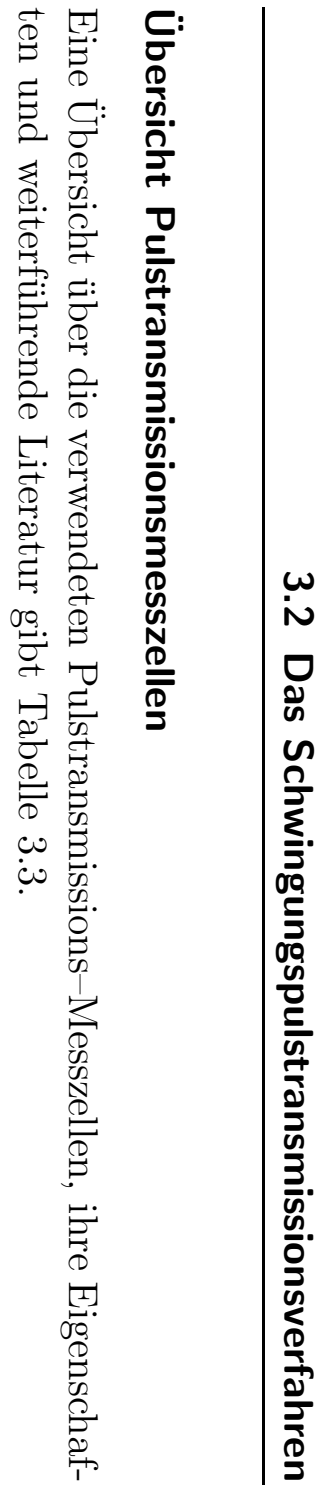




\subsubsection{Fehlerdiskussion für das Pulstransmissionsverfahren}

Die folgende Aufzählung stellt die wichtigsten Fehlerursachen des Pulstransmissionsverfahrens dar; Tabelle 3.4 gibt eine Abschätzung der relativen Gesamtfehler bei für Kohlenhydratlösungen typischen Absorptionen wieder.

Frequenzfehler: Der Fehler in der Sendefrequenz kann bei Verwendung von Synthetisiersendern vernachlässigt werden ( $\mathrm{f}<2 \mathrm{GHz}$ ).

Temperaturstabilitat: Die Temperaturschwankungen während einer Messung betrugen maximal 0,02 K, die Abweichung von der Solltemperatur laut Digitalthermometer max. 0,03 K. Allerdings kann diese Abweichung, insbesondere bei den Messungen bei $15^{\circ} \mathrm{C}$ und $35^{\circ} \mathrm{C}$ etwas höher liegen, da die Temperaturmessung nicht direkt zwischen den Wandlern erfolgen kann, sondern am inneren Zellmantel durchgeführt wurde. Die Temperaturschwankungen wirken sich auf den Dämpfungsexponenten sowohl über die Hintergrunddämpfung, wie auch über die Beeinflussung der Relaxationsprozesse aus. Der Fehler kann mit 0,15 \% abgeschätzt werden [27].

A btastpuls: Einen merklichen systematischen Fehler erhält man bei einer nicht hinreichend genauen Bestimmung der Schallgeschwindigkeit zur Nachführung des Abtastpulses. Da sich dieser meist in einer Abweichung vom exponentiellen Abklingverhalten bemerkbar macht, kann er durch sorgfältige Kontrolle der Anpassung erkannt und weitestgehend vermieden werden.

B eugungskorrektur: Am tieffrequenten Ende des Frequenzintervalls der jeweiligen Messzelle verfälschen Beugungseffekte den Dämpfungsexponenten, welche die Korrektur nicht vollständig ausgleichen kann. Daher ist der Messfehler hier gegenüber dem bei mittleren Frequenzen der Messzelle etwas erhöht.

Gesamtdampfung: Wiederum im tieffrequenten Teil des Messbereichs spielt der begrenzte zur Verfügung stehende Verfahrweg eine Rolle. Hier wird, insbesondere bei den 1-MHz-Pulszellen und der PZT-Zelle, ein Abfall über die Messstrecke von z.T. nur wenig mehr als 3 dB erreicht. Dadurch erhöht sich der Fehler gegenüber der Messung bei dem sonst angestrebten 15-18 dB Abfall. Insbesondere in diesen Frequenzbereichen müssen Abweichungen von einem exponentiellen Abklingverhalten minimiert werden, um zufriedenstellende Fehler in den $\alpha$-Werten zu erreichen.

Paralleljustierung: Gerade bei den langen Fahrwegen der 1-MHz-Pulszellen kann eine mangelnde Paralleljustierung der Wandler zu einem systematischen Fehler führen, da dann der Empfangswandler zunehmend aus der Achse des Schallstrahls herausfährt und somit eine zu große Absorption gemessen wird.

Welligkeiten: Zum Teil mussten Messungen bei auftretender $\lambda / 2-$ und $\lambda$-Welligkeit ausgeführt werden. Diese konnten im Allgemeinen beim Anpassen der Übertragungfunktion berücksichtigt werden. In Fällen, in denen die Modulation nicht hinreichend ausgeprägt war, liefert der nichtlineare Anpassalgorithmus allerdings physikalisch unsinnige Parameter, so dass dann auf die lineare Regression zurückgegriffen werden musste.

Erwarmung: Eine Erwärmung der Messlösung durch Schallabsorption kann bei hohen Dämpfungen nicht ausgeschlossen werden, wurde jedoch bei den Kohlenhydratlösungen nicht beobachtet.

Da sich eine Bestimmung des Gesamtfehlers aufgrund der oben genannten Ursachen in den Frequenzbereichen der verschiedenen Pulszellen schwierig gestaltet, und die einzelnen Fehler sich kompensieren können, wurden zur Fehlerabschätzung Mehrfachmessungen an ausgewählten Saccharidlösungen herangezogen. Hierbei wurden 
jeweils frisch angesetzte Lösungen verwendet, wobei die Kohlenhydrate zum Teil aus verschiedenen Produktionschargen oder von unterschiedlichen Herstellern stammten. Außerdem wurden die Zellen vor jeder Messung erneut gereinigt. Schliesslich bietet auch der Überlappungsbereich von Messungen mit den verschiedenen Pulszellen bzw. mit den Resonatoren die Möglichkeit, den Fehler in den Randbereichen der Messfrequenzintervalle abzuschätzen.

\begin{tabular}{|c|c|c|c|c|c|c|}
\hline Zelle & \multicolumn{3}{|c|}{$\begin{array}{c}\text { Frequenzintervall } \\
f \\
{[\mathrm{MHz}]}\end{array}$} & \multicolumn{2}{|c|}{$\begin{array}{c}\text { Absorption } \\
\alpha / f^{2} \\
{\left[10^{-15} \mathrm{~s}^{2} / \mathrm{m}\right]}\end{array}$} & $\begin{array}{c}\text { Fehler } \\
\Delta \alpha / \alpha \\
\%\end{array}$ \\
\hline $1-\mathrm{MHz}^{-}$ & 3 & $\leq f \leq$ & 100 & $100 \leq\left(\alpha / f^{2}\right)$ & $\leq 1000$ & 1,5 \\
\hline Pulszellen & 13 & $\leq f \leq$ & 100 & $\left(\alpha / f^{2}\right)$ & 100 & 2,0 \\
\hline $10-\mathrm{MHz}^{-}$ & 30 & $\leq f \leq$ & 50 & & & 1,5 \\
\hline Pulszelle & 50 & $\leq f \leq$ & 400 & & & 0,5 \\
\hline & 400 & $\leq f \leq$ & 520 & & & 1,5 \\
\hline Hyperschallzelle & 520 & $\leq f \leq$ & 2000 & & & 1,5 \\
\hline PZT-Zelle & 600 & $\leq f \leq$ & 1100 & $\leq\left(\alpha / f^{2}\right)$ & & 1,5 \\
\hline & 1100 & $\leq f \leq$ & 2000 & & & 1,0 \\
\hline
\end{tabular}

Tabelle 3.4: Relativer Gesamtfehler in den Frequenzbereichen der verschiedenen Pulszellen. Die Fehler wurden aus Mehrfachmessungen an Wasser und Sacchariden sowie nach [28] [12] [27] abgeschätzt. 


\section{Präparation der Messlösungen}

Die Bezugsquellen der verwendeten Chemikalien und ihre Reinheitsgrade sind in Tabelle 4.1 aufgeführt. Zusätzlich ist der systematische Name der Disaccharide angegeben, da sich hieran die Konstitutionen der Moleküle - insbesondere die beteiligten Monosaccharide und ihre Verknüpfung - ablesen lassen. Die Substanzen wurden, wie vom Hersteller geliefert, ohne weitere Reinigung oder Trocknung verwendet.

Das Wasser zum Ansetzen der Lösungen wurde entweder deionisiert, bidestilliert und UV-bestrahlt oder in einem mehrstufigen Ionentauscher Millipore „Milli-Q“ gereinigt. Zusätzlich wurde das Wasser zum Entgasen und Keimabtöten abgekocht.

Die Feststoffe wurden auf Analysewaagen (Sartorius BP 3100 S mit einer Genauigkeit von 0,01 g und Sartorius 2258 mit 1/1000 g Genauigkeit) in einen Erlenmeyerkolben eingewogen und ein Teil des Wassers hinzugegeben. Um den Lösungsvorgang zu beschleunigen, wurden insbesondere die höher konzentrierten Lösungen im Wasserbad auf ca. $50^{\circ} \mathrm{C}$ erwärmt, mit einem Magnetrührer durchmischt und zusätzlich in ein Ultraschallbad gehängt. Im Folgenden wurde die Lösung in einen Messkolben umgefüllt, der Erlenmeyerkolben nachgespült und Wasser bis auf den Eichstrich des Messkolbens hinzugegeben. Hierbei wurde der Kolben auf die Eichtemperatur von $20^{\circ} \mathrm{C}\left( \pm 0,5^{\circ} \mathrm{C}\right)$ thermostatiert und Luftblasen im Ultraschallbad entfernt. Die angegebenen Molaritäten beziehen sich mithin auf $20^{\circ} \mathrm{C}$. Die erhaltenen Kohlenhydratlösungen waren klar, bei hohen Konzentrationen sehr leicht bräunlich; die Harnstofflösungen waren bis zur höchsten Konzentration klar.

Die Ansätze wurden im Kühlschrank bei ca. $5-8^{\circ} \mathrm{C}$ gelagert und nach spätestens 14 Tagen nicht mehr verwendet, da Zuckerlösungen einen guten Nährboden für das Wachstum von Mikroorganismen bieten. Von einem Giftzusatz zur Keimabtötung wurde abgesehen.

Zwischen dem Ansetzen und der ersten Messung lagen mindestens zwölf Stunden, da auch einige der Disaccharide eine langsame Mutarotation zeigen (vgl. Abschnitt 6.2) und auf die Einstellung des Gleichgewichts gewartet wurde.

Vor jeder Messung wurden die Lösungen erneut unter Vakuum oder im Ultraschallbad entgast, auf evtl. sichtbare Veränderungen überprüft und auf die Messtemperatur vorthermostatiert.

Die Dichten der Lösungen wurden mit einem Pyknometer, die Scherviskositäten mit einem Kapillar- (Schott) oder Kugelfallviskosimeter (Haake) bestimmt und können Tabelle 4.2 entnommen werden. Die ebenfalls angegebenen Schallgeschwindigkeiten der Saccharidlösungen sind die Mittelwerte der Messungen mit den hochund tieffrequenten Resonatoren. 


\begin{tabular}{|c|c|c|c|c|c|c|}
\hline Substanz & systematische Nomenklatur & Trivialname & Summenformel & Hersteller & Reinheit & $\mathrm{M}_{r}$ \\
\hline $\begin{array}{l}\mathrm{D}(+)-\text { Maltose } \\
\text { Monohydrat }\end{array}$ & $\begin{array}{l}4-\mathrm{O}- \\
(\alpha-\mathrm{D}-\text { Glucopyranosyl })^{-} \\
\text {D-Glucopyranose }\end{array}$ & Malzzucker & $\mathrm{C}_{12} \mathrm{H}_{22} \mathrm{O}_{11} \cdot \mathrm{H}_{2} \mathrm{O}$ & Fluka & $\geq 99 \%$ & 360,32 \\
\hline $\begin{array}{l}\mathrm{D}(+) \text {-Trehalose } \\
\text { Dihydrate }\end{array}$ & $\begin{array}{l}\alpha-\text { D-Glucopyranosyl-- } \\
\alpha-\text { D-Glucopyranosid } \\
\end{array}$ & & $\mathrm{C}_{12} \mathrm{H}_{22} \mathrm{O}_{11} \cdot 2 \mathrm{H}_{2} \mathrm{O}$ & Fluka & $\geq 99 \%$ & 378,34 \\
\hline $\begin{array}{l}\mathrm{D}(+)-\text { Lactose } \\
\text { Monohydrat }\end{array}$ & $\begin{array}{l}4-\mathrm{O}- \\
(\beta-\mathrm{D}-\text { Galactopyranosyl)- } \\
\mathrm{D}-\text { Glucopyranose }\end{array}$ & Milchzucker & $\mathrm{C}_{12} \mathrm{H}_{22} \mathrm{O}_{11} \cdot \mathrm{H}_{2} \mathrm{O}$ & Fluka & $\geq 99 \%$ & 360,32 \\
\hline $\begin{array}{l}\mathrm{D}(+) \text {-Melibiose } \\
\text { Monohydrat }\end{array}$ & $\begin{array}{l}6-\mathrm{O}- \\
(\alpha-\mathrm{D}-\text { Galactopyranosyl)- } \\
\mathrm{D}-\text { Glucopyranose }\end{array}$ & & $\mathrm{C}_{12} \mathrm{H}_{22} \mathrm{O}_{11} \cdot \mathrm{H}_{2} \mathrm{O}$ & Merck & $\geq 98 \%$ & 360,32 \\
\hline $\begin{array}{l}\mathrm{D}(+) \text {-Sucrose } \\
\mathrm{D}(+) \text {-Saccharose }\end{array}$ & $\begin{array}{l}\beta \beta-\text { D-Fructofuranosyl- } \\
\alpha-\text { D-Glucopyranosid }\end{array}$ & Rohrzucker & $\mathrm{C}_{12} \mathrm{H}_{22} \mathrm{O}_{11}$ & Fluka & $\geq 99,5 \%$ & 342,30 \\
\hline $\mathrm{D}(-)-$ Fructose & & Fruchtzucker & $\mathrm{C}_{6} \mathrm{H}_{12} \mathrm{O}_{6}$ & Sigma & $\geq 99 \%$ & 180,02 \\
\hline $\begin{array}{l}\text { Calciumchlorid } \\
\text { Dihydrat }\end{array}$ & & & $\mathrm{CaCl}_{2} \cdot 2 \mathrm{H}_{2} \mathrm{O}$ & Fluka & $\geq 99 \%$ & 147,02 \\
\hline Urea & & Harnstoff & $\mathrm{OC}\left(\mathrm{NH}_{2}\right)_{2}$ & Fluka & $\geq 99,5 \%$ & 60,06 \\
\hline
\end{tabular}




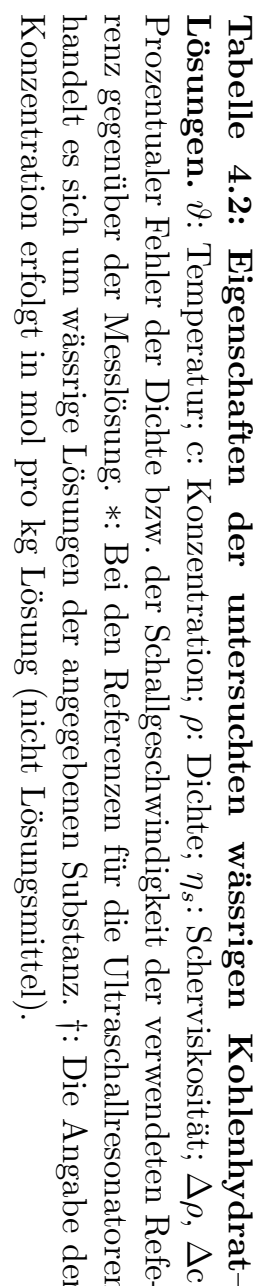

\begin{tabular}{|c|c|c|c|c|c|c|c|c|c|c|c|c|}
\hline Lösung & $\begin{array}{l}\vartheta \\
{ }^{\circ} \mathrm{C}\end{array}$ & $\begin{array}{c}\mathrm{c} \\
{[\mathrm{mol} / \mathrm{l}]}\end{array}$ & $\begin{array}{c}\rho \\
{[\mathrm{g} / \mathrm{ml}]}\end{array}$ & $\begin{array}{c}\eta_{s} \\
{[\mathrm{mPas}]}\end{array}$ & $\begin{array}{c}\mathrm{c}_{s} \\
{[\mathrm{~m} / \mathrm{s}]}\end{array}$ & Referenz & $\begin{array}{c}\mathrm{c} \\
{[\mathrm{mol} / \mathrm{kg}]^{\dagger}}\end{array}$ & $\begin{array}{c}\mathrm{c} \\
{[\mathrm{mol} / \mathrm{l}]}\end{array}$ & $\begin{array}{c}\rho \\
{[\mathrm{g} / \mathrm{ml}]}\end{array}$ & $\begin{array}{c}\mathrm{c}_{s} \\
{[\mathrm{~m} / \mathrm{s}]}\end{array}$ & $\begin{array}{c}\Delta \rho \\
\%\end{array}$ & $\begin{array}{c}\Delta \mathrm{c} \\
\%\end{array}$ \\
\hline \multirow[t]{8}{*}{ Maltose } & $\overline{10}$ & 0,5 & $\overline{1,0670}$ & 22,440 & 1509,75 & Urea & 1,9140 & $\overline{~ 1,9756}$ & $\overline{1,0322}$ & $\overline{1511,03}$ & $\overline{3,26}$ & $\overline{0,08}$ \\
\hline & 15 & 0,5 & 1,0660 & 2,196 & 1525,22 & Urea & 1,9140 & 1,9736 & 1,0311 & 1524,57 & 3,27 & 0,04 \\
\hline & 25 & 0,5 & 1,0632 & 1,694 & 1551,52 & Methanol & - & 4,2643 & 0,9726 & 1548,88 & 8,52 & 0,17 \\
\hline & 35 & 0,5 & 1,0592 & 1,317 & 1569,20 & Urea & 2,2007 & 2,2639 & 1,0287 & 1569,26 & 6,18 & 0,004 \\
\hline & 25 & 1 & 1,1292 & 2,963 & 1613,68 & Urea & 4,4790 & 4,7985 & 1,0713 & 1613,76 & 5,13 & 0,01 \\
\hline & 25 & 1,5 & 1,1930 & 6,997 & 1686,07 & Urea & 7,2549 & 8,1244 & 1,1199 & 1686,09 & 6,13 & 0,001 \\
\hline & 40 & 1,5 & 1,1881 & 4,437 & 1697,59 & Urea & 7,6817 & 8,5915 & 1,1184 & 1694,66 & 5,87 & 0,17 \\
\hline & 25 & 1,8 & 1,2320 & 13,773 & 1733,98 & Urea & 9,0578 & 10,4381 & 1,1524 & 1733,74 & 6,46 & 0,01 \\
\hline \multirow[t]{4}{*}{ Trehalose } & 10 & 1 & 1,1401 & 5,311 & 1594,5 & Urea & 4,4936 & 4,8401 & 1,0771 & 1593,52 & 5,53 & 0,06 \\
\hline & 15 & 1 & 1,1386 & 5,242 & 1606,7 & Urea & 4,6411 & 5,0037 & 1,0781 & 1604,96 & 5,31 & 0,11 \\
\hline & 25 & 1 & 1,1355 & 3,185 & 1625,61 & Urea & 4,9344 & 5,3251 & 1,0792 & 1623,98 & 4,96 & 0,10 \\
\hline & 35 & 1 & 1,1298 & 2,381 & 1640,51 & Urea & 5,2987 & 5,7232 & 1,0801 & 1639,84 & 4,40 & 0,04 \\
\hline \multirow[t]{3}{*}{ Lactose } & 18 & 0,5 & 1,0665 & 1,898 & 1538,69 & Urea & 2,1527 & 2,2268 & 1,0344 & 1539,05 & 3,01 & 0,02 \\
\hline & 25 & 0,5 & 1,0653 & 1,549 & 1555,27 & Urea & 2,2459 & 2,3216 & 1,0337 & 1555,16 & 2,97 & 0,01 \\
\hline & 35 & 0,5 & 1,0616 & 1,215 & 1574,1 & Urea & 2,4164 & 2,4942 & 1,0322 & 1573,85 & 2,77 & 0,02 \\
\hline \multirow[t]{4}{*}{ Melibiose } & 10 & 0,5 & 1,0707 & 2,350 & 1518,45 & Urea & 2,1781 & 2,2581 & 1,0367 & 1520,1 & 3,18 & 0,11 \\
\hline & 15 & 0,5 & 1,0696 & 2,033 & 1533,21 & Urea & 2,1781 & 2,2557 & 1,0356 & 1533,27 & 3,18 & 0,004 \\
\hline & 25 & 0,5 & 1,067 & 1,563 & 1557,89 & Urea & 2,3464 & 2,4294 & 1,0354 & 1556,47 & 2,96 & 0,09 \\
\hline & 35 & 0,5 & 1,0642 & 1,194 & 1576,33 & Urea & 2,5147 & 2,5997 & 1,0338 & 1574,95 & 2,86 & 0,09 \\
\hline Sucrose & 25 & 1 & 1,1295 & 2,348 & 1608,05 & Urea & 4,0866 & 4,3509 & 1,0687 & 1609,84 & 5,38 & 0,11 \\
\hline \multirow{3}{*}{$+\mathrm{CaCl}_{2}$} & 25 & $0,5 / 0,25$ & 1,0857 & 1,690 & 1574,99 & Urea & 3,0008 & 3,1396 & 1,0463 & 1574,07 & 3,63 & 0,06 \\
\hline & 25 & $0,5 / 0,5$ & 1,1062 & 1,809 & 1600,78 & Urea & 3,9864 & 4,2372 & 1,0629 & 1600,31 & 3,91 & 0,03 \\
\hline & 25 & $0,5 / 1$ & 1,1489 & 2,237 & 1645,67 & Urea & 5,6994 & 6,2262 & 1,0924 & 1645,48 & 4,92 & 0,01 \\
\hline
\end{tabular}




\section{Urea: Referenz für Ultraschallresonatormessungen}

Bei der Bestimmung der Absorption einer Ultraschallwelle mittels des in Abschnitt 3.1 beschriebenen Resonatorverfahrens besteht das Problem, den Anteil der Flüssigkeitsdämpfung von den apparativen Verlusten zu trennen. Gleichung (3.10) gibt eine theoretische Beschreibung des Beugungsanteils an der Gesamtdämpfung, kann damit allerdings nicht alle Absorptionsursachen erfassen. Damit ist eine Berechnung aus den geometrischen Daten und den Materialeigenschaften des Resonators sowie der Dichte und Schallgeschwindigkeit der Messlösung nicht ausreichend, um die Flüssigkeitsdämpfung zu ermitteln. Vielmehr müssen die apparativen Verluste für jede Messlösung durch eine Referenzmessung mit einer Flüssigkeit/Lösung, die die gleichen Eigenschaften besitzt, experimentell bestimmt werden. Nach Gleichung (3.10) folgen damit als Anforderungen an die Referenz, dass insbesondere die Schallgeschwindigkeiten und die Dichten übereinstimmen müssen. Zusätzlich wird der Dämpfungsexponent der Referenz benötigt, da deren Flüssigkeitsabsorption ebenfalls in den Messungen enthalten ist. In Bezug auf wässrige Lösungen von Kohlenhydraten bedeutet dies, dass man die Schallgeschwindigkeit der Referenz auf Werte zwischen ca. $1500 \mathrm{~m} / \mathrm{s}$ und $1735 \mathrm{~m} / \mathrm{s}$ und die Dichte zwischen $1 \mathrm{~g} / \mathrm{ml}$ und 1,25 g/ml einstellen können sollte. Aufgrund vorangegangener breitbandiger Absorptionsmessungen von Rupprecht [27] [32] und Bödecker [33] erschienen wässrige Harnstofflösungen als Referenzsystem geeignet. Wie die nachfolgende Abbildung (5.1) der Ultraschallspektren von Rupprecht zeigt, treten bei Harnstofflösungen bis zur höchsten gemessenen Konzentration von $9 \mathrm{~mol} / \mathrm{l}$ im Frequenzbereich bis ca. $200 \mathrm{MHz}$ keine Relaxationsstufen auf. Daher ist es möglich, die Absorption der jeweiligen Harnstofflösung für den Resonatorbereich direkt - ohne die Notwendigkeit einer weiteren Referenzflüssigkeit - höherfrequent mit dem Pulstransmissionsverfahren zu messen. Damit für die sowohl konzentrations- wie auch temperaturabhängigen Messungen an wässrigen Kohlenhydratlösungen jeweils eine geeignete Referenz zur Verfügung stand, wurden die Dichten, Schallgeschwindigkeiten und Dämpfungsexponenten von Harnstoffösungen im Konzentrationsbereich von 0 bis $9,07 \mathrm{~mol} / \mathrm{l}$ bei Temperaturen von $10^{\circ} \mathrm{C}$ bis $35^{\circ} \mathrm{C}$ gemessen. Die Absorption wurde mit der $10-\mathrm{MHz}-\mathrm{Pulszelle}$ (siehe Abschnitt 3.2.8) im Frequenzbereich geringster Messfehler der Apparatur von 100 bis $210 \mathrm{MHz}$ bestimmt. Die Schallgeschwindigkeit wurde nach Gleichung (3.13) aus den Resonanzabständen der Zellresonanzen des 4-MHz-Resonators (siehe Abschnitt 3.1) berechnet. Da hierbei weder Beugungsverluste noch Nebenmodeneinflüsse noch der Effekt der Quarzresonanzen berücksichtigt werden, wurden nur die Frequenzbereiche von 2,5 bis $3 \mathrm{MHz}$ und von 4,5 bis $6 \mathrm{MHz}$ genutzt. Die Dichten wurden mit Hilfe eines Pyknometers bestimmt, und deren relativer Fehler aus Erfahrungswerten mit ähnlichen Lösungen mit 0,033 \% abgeschätzt. Die Ergebnisse sind in der nachfolgende Tabelle 5.1 enthalten. 


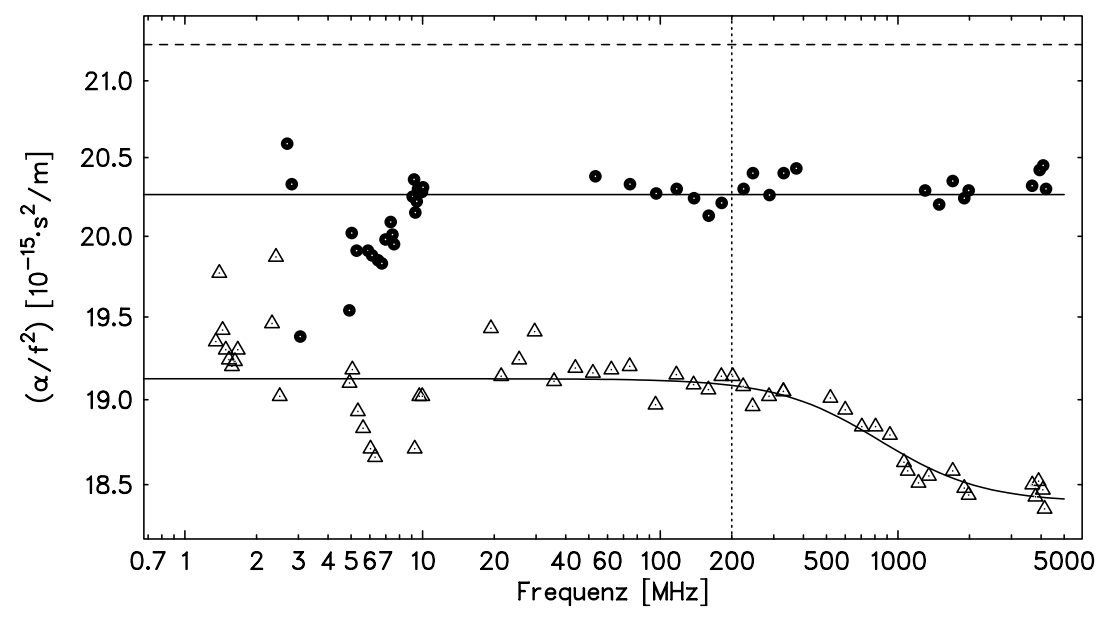

\begin{abstract}
Abbildung 5.1: $\left(\alpha / f^{2}\right)$-Spektren für 1,01(•) und 9,06( $\left.\triangle\right)$ molare wässrige Harnstofflösungen. Im Fall der 1,01 molaren Lösung wurde nur der Hintergrundanteil angepasst (keine Relaxationstufe messbar), an die Messdaten der 9,06 molaren Lösung wurde zur Veranschaulichung die Summe aus dem Hintergrundanteil und einer Debye-Spektralfunktion angepasst (in [32] wurde ein integratives KonzentrationsFluktuationsmodell verwendet). Zum Vergleich gibt die gestrichelte Linie die klassische Dämpfung des reinen Wassers $\left(\alpha / f^{2}=21,24 \cdot 10^{-15} \mathrm{~s}^{2} / \mathrm{m}\right)$ wieder.
\end{abstract}

Harnstoff-Lösungen bieten im Hinblick auf die Messung von Saccharidlösungen gegenüber der bisher verwendetet Referenz aus Wasser-Methanol den Vorteil, dass die maximal erreichbare Schallgeschwindigkeit bei $25^{\circ} \mathrm{C}$ ca. $1740 \mathrm{~m} / \mathrm{s}$ beträgt (WasserMethanol: ca. $1570 \mathrm{~m} / \mathrm{s}$ ) und dass auch temperaturabhängig, insbesondere zu höheren Temperaturen, gemessen werden kann. Bei Wasser-Methanol-Lösungen lassen sich Konzentrationsänderungen aufgrund des tiefen Siedepunktes des Methanols von $64,5^{\circ} \mathrm{C}$ schon bei Raumtemperatur nur schwer vermeiden. Nicht zuletzt ist Harnstoff weit weniger gesundheitsschädlich als Methanol.

Mit Hilfe des binären Systems Harnstoff-Wasser lässt sich nur einer der Parameter, die die apparativen Verluste mitbestimmen, genau einstellen. Hier wird die Schallgeschwindigkeit gewählt, zum einen aufgrund der quadratischen Abhängigkeit der Beugungsverluste von der Schallgeschwindigkeit (siehe Abbildung 5.2), zum anderen um sicherzustellen, dass die Zellresonanzen der Messung und der Referenzmessung bei den gleichen Frequenzen liegen. Weiterhin beträgt die Dichte der HarnstoffLösung an der Löslichkeitsgrenze bei $25^{\circ} \mathrm{C}$ ca. $1,16 \mathrm{~g} / \mathrm{ml}$ und bietet damit keinen hinreichenden Variationsbereich.

Insgesamt sind Harnstoff-Lösungen als Referenz für Messlösungen mit Schallgeschwindigkeiten von $1496 \mathrm{~m} / \mathrm{s}$ bis $1740 \mathrm{~m} / \mathrm{s}$ und Dichten größer als die von Wasser einsetzbar, während Methanol-Wasser für den Schallgeschwindigkeitsbereich von $1100 \mathrm{~m} / \mathrm{s}-1570 \mathrm{~m} / \mathrm{s}$ und Dichten unter der von Wasser geeignet ist. Ein weiteres, früher vielfach benutztes Referenzsystem aus wässrigen Kochsalzlösungen hat den grundlegenden Nachteil, dass es die Bedampfung der Wandler chemisch angreift und sollte daher nicht mehr verwendet werden.

Im Folgenden wurden an die Messdaten für die Dämpfung, Schallgeschwindigkeit und Dichte Polynome zweier Variabler, Konzentration $c$ und Temperatur $\theta$, angepasst, damit für eine Messlösung bei gegebener Temperatur eine Referenz mit 


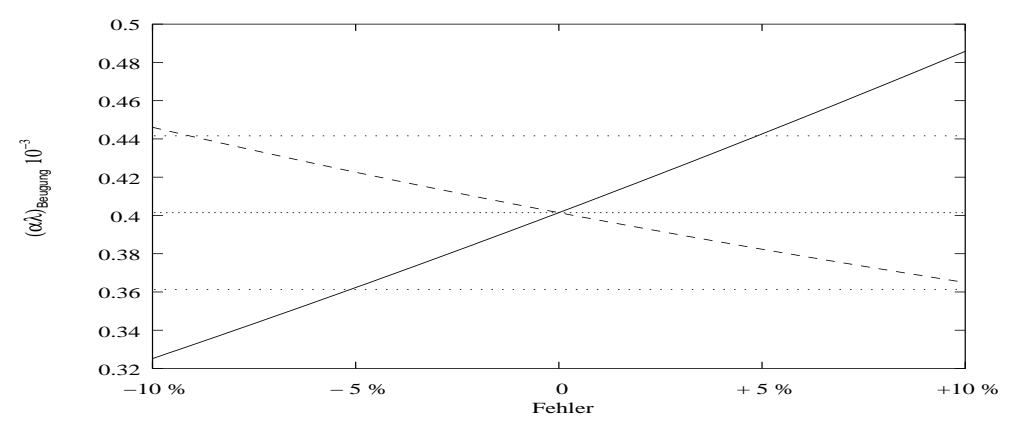

\begin{abstract}
Abbildung 5.2: Berechnete Dämpfung längs einer Wellenlänge aufgrund von Beugung bei einer Frequenz von $200 \mathrm{kHz}$ für eine Referenzlösung in einem Resonator mit PVC-Zellberandung (70 mm Durchmesser). Auf der Abszisse ist der Fehler

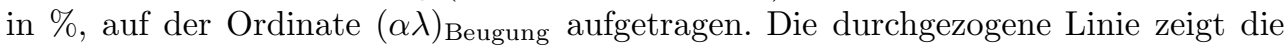

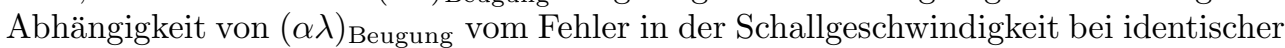
Dichte von Mess- und Referenzlösung, die gestrichelte Linie entsprechend $(\alpha \lambda)_{\text {Beugung }}$ als Funktion vom Fehler in der Dichte bei identischer Schallgeschwindigkeit. Die gepunkteten Linien geben den Wert für die Messlösung und die $10 \%$ Fehlergrenzen wieder.
\end{abstract}

gleicher Schallgeschwindigkeit ermittelt werden kann. Dabei ist zu betonen, dass die verwendeten Polynome keinem physikalischen Modell folgen, sondern lediglich die Messdaten im vorgegebenen Gebiet so gut wie möglich beschreiben sollen. Es wurden die folgenden Polynome verwendet; die Anpassung mit dem MarquardtLevenberg-Algorithmus (Programm: gnuplot) ergab die aufgelisteten Parameter:

\title{
a) Fur die Schallgeschwindigkeit:
}

$$
f(c, \theta)=a \cdot \theta^{3}+b \cdot c^{2} \cdot \theta+z \cdot c^{2}+d \cdot c+e \cdot \theta^{2}+f \cdot \theta+g \cdot c \cdot \theta+h+k \cdot c \cdot \theta^{2}
$$

mit: $c$ : Konzentration; $\theta$ : Temperatur.

Parameter:

$$
\begin{array}{ll}
\mathrm{a}=0,00019(15) & \mathrm{b}=0,0096(10) \\
\mathrm{z}=-0,223(26) & \mathrm{d}=39,15(23) \\
\mathrm{e}=-0,0525(99) & \mathrm{f}=4,94(21) \\
\mathrm{g}=-0,622(18) & \mathrm{h}=1403(1) \\
\mathrm{k}=0,00392(38) &
\end{array}
$$

\section{b) Fur die Dampfung:}

$$
f(c, \theta)=a \cdot c^{2} \cdot \theta^{2}+b \cdot c^{2} \cdot \theta+z \cdot c^{2}+d \cdot c+e \cdot \theta^{2}+f \cdot \theta+g \cdot c \cdot \theta+h+k \cdot c \cdot \theta^{2}
$$

mit: $c$ : Konzentration; $\theta$ : Temperatur.

Parameter:

$$
\begin{array}{ll}
\mathrm{a}=0,000175(85) & \mathrm{b}=-0,0128(44) \\
\mathrm{z}=0,319(53) & \mathrm{d}=-4,22(35) \\
\mathrm{e}=0,01796(58) & \mathrm{f}=-1,566(27) \\
\mathrm{g}=0,186(30) & \mathrm{h}=49,08(26) \\
\mathrm{k}=-0,00250(57) &
\end{array}
$$




\title{
c) Fur die Dichte:
}

$$
f(c, \theta)=a \cdot c^{2}+b \cdot c+z \cdot \theta+d \cdot c \cdot \theta+e
$$

mit: $c$ : Konzentration; $\theta$ : Temperatur.

Parameter:

$$
\begin{array}{ll}
\mathrm{a}=1,13(11) \cdot 10^{-4} & \mathrm{~b}=0,0171(1) \\
\mathrm{z}=-2,81(14) \cdot 10^{-4} & \mathrm{~d}=-4,23(29) \cdot 10^{-5} \\
\mathrm{e}=1,0039(4) &
\end{array}
$$

Für alle in dieser Arbeit untersuchten Disaccharid-Lösungen wurde jeweils die Referenz so gewählt, dass die Schallgeschwindigkeiten von Mess- und Referenzlösungen so gut wie möglich übereinstimmen, die maximale Abweichung in der Schallgeschwindigkeit beträgt dabei ca. 0,2 \%, die durchschnittliche 0,05\%. Die Dichte der Referenz war damit ebenfalls festgelegt, hier ergab sich eine maximale Differenz von $6,5 \%$, bei einem Durchschnitt von 4,3\% Fehler (vgl. Tabelle 4.2).

Interessant ist die untypisch hohe Löslichkeit des organischen Moleküls Harnstoff in Wasser, das sich sehr gut in dessen Wasserstoffbrücken-Struktur einpasst. Infrarot-spektroskopische Messungen ergeben, dass Harnstoff nur die unmittelbare Hydrathülle in ihren Eigenschaften beeinflusst [34]. Berechnet man aus den Dichten $\rho$ und Schallgeschwindigkeiten $c_{s}$ die adiabatische Kompressibilität $\kappa_{s}$ der Harnstofflösungen nach der Newton-Laplace-Gleichung $\kappa_{s}=1 / \rho c_{s}^{2}$ [35] so erhält man für ausgewählte Konzentrationen den in Abbildung 5.3 dargestellten Verlauf über der Temperatur. Zur besseren Veranschaulichung wurden die Werte auf $\kappa_{s}$ für $25^{\circ} \mathrm{C}$ skaliert. Man erkennt, dass der anomale Kompressibilitätsverlauf des Wassers - die

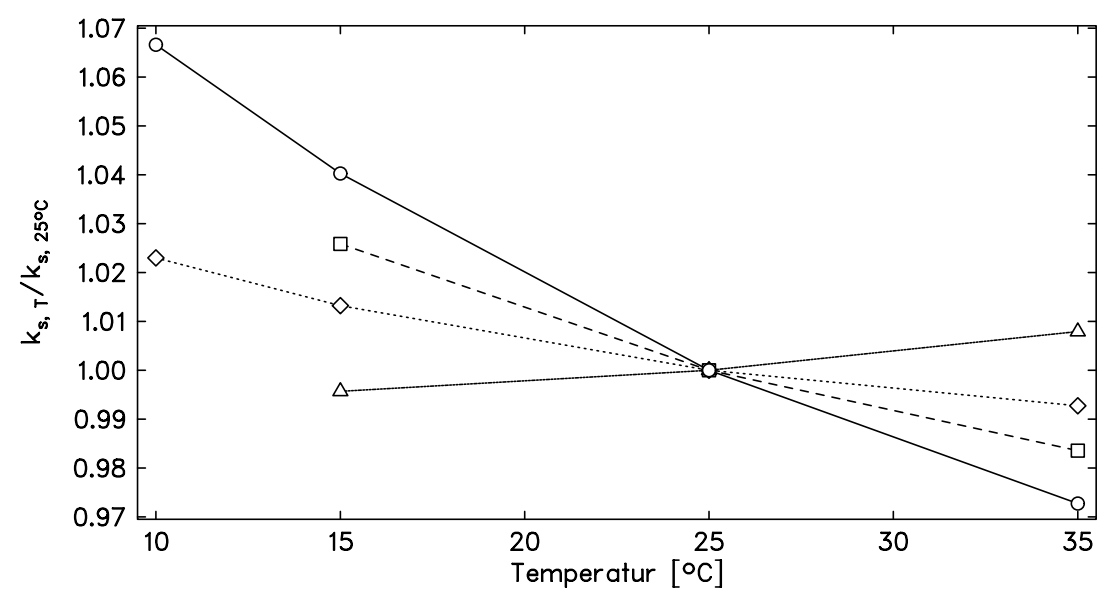

\begin{abstract}
Abbildung 5.3: Vergleich des Kompressibilitätsverlaufs über der Temperatur für reines Wasser (॰), $2 \mathrm{~mol} / \mathrm{kg}(\square), 4 \mathrm{~mol} / \mathrm{kg}(\diamond)$ und $8 \mathrm{~mol} / \mathrm{kg}$ Harnstofflösung $(\triangle)$. Die Kompressibilitäten sind auf den Wert für $25^{\circ} \mathrm{C}$ skaliert.
\end{abstract}

Kompressibilität nimmt bis ca. $75^{\circ} \mathrm{C}$ ab - bereits bei der kleinsten gemessenen Konzentration von $2 \mathrm{~mol} / \mathrm{kg}$ merklich beeinflusst wird. Bei $8 \mathrm{~mol} / \mathrm{kg}$ liegt im angegebenen Temperaturbereich wieder normales Verhalten vor. 


\begin{tabular}{|c|c|c|c|c|c|c|c|}
\hline 局 & $\begin{array}{l}\text { Konzentration } \\
{[\mathrm{mol} / \mathrm{kg}]^{a}}\end{array}$ & $\begin{array}{l}\text { Konzentration } \\
{[\mathrm{mol} / \mathrm{l}]}\end{array}$ & $\begin{array}{c}\text { Temperatur } \\
{\left[{ }^{\circ} \mathrm{C}\right]}\end{array}$ & $\begin{array}{c}\text { Dämpfung } \\
10^{-15}\left[\mathrm{~s}^{2} / \mathrm{m}\right]\end{array}$ & $\begin{array}{l}\text { Dichte } \\
{[\mathrm{g} / \mathrm{ml}]}\end{array}$ & $\begin{array}{l}\text { Schallgeschwindigkeit } \\
{[\mathrm{m} / \mathrm{s}]}\end{array}$ & $\begin{array}{c}\text { Kompressibilität } \\
10^{-10}\left[\mathrm{~Pa}^{-1}\right]\end{array}$ \\
\hline 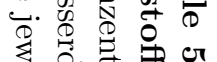 & $0^{b}$ & 0 & 10 & $35,32(3)$ & $0,9997(3)$ & $1447,28(5)$ & $4,776(2)$ \\
\hline 苞: & $0^{b}$ & 0 & 15 & $29,46(3)$ & $0,9991(3)$ & $1465,94(5)$ & $4,658(2)$ \\
\hline & $0^{b}$ & 0 & 25 & $21,28(3)$ & $0,9971(3)$ & $1496,70(5)$ & $4,477(2)$ \\
\hline & $0^{b}$ & 0 & 35 & $16,30(3)$ & $0,9940(3)$ & $1519,83(5)$ & $4,355(1)$ \\
\hline & 2 & 2,065 & 15 & $26,21(8)$ & $1,0324(3)$ & $1527,19(7)$ & $4,153(1)$ \\
\hline 告 & 2 & 2,059 & 25 & $19,36(5)$ & $1,0297(3)$ & $1548,88(9)$ & $4,048(1)$ \\
\hline Us & 2 & 2,051 & 35 & $15,07(2)$ & $1,0257(3)$ & $1564,80(8)$ & $3,982(1)$ \\
\hline & 3 & 3,148 & 15 & $25,02(8)$ & $1,0492(4)$ & $1557,18(8)$ & $3,931(1)$ \\
\hline & 3 & 3,139 & 25 & $18,78(5)$ & $1,0465(4)$ & $1574,77(10)$ & $3,853(1)$ \\
\hline อ & 3 & 3,124 & 35 & $14,78(4)$ & $1,0413(3)$ & $1587,14(11)$ & $3,812(1)$ \\
\hline . & 4 & 4,275 & 10 & $28,43(13)$ & $1,0687(4)$ & $1578,82(11)$ & $3,754(1)$ \\
\hline . & 4 & 4,269 & 15 & $24,25(13)$ & $1,0672(4)$ & $1587,58(8)$ & $3,718(1)$ \\
\hline & 4 & 4,253 & 25 & $18,41(8)$ & $1,0631(4)$ & $1601,07(9)$ & $3,669(1)$ \\
\hline & 4 & 4,232 & 35 & $14,60(4)$ & $1,0579(4)$ & $1610,87(10)$ & $3,643(1)$ \\
\hline 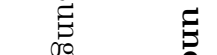 & 5 & 5,421 & 15 & $23,85(14)$ & $1,0843(4)$ & $1617,02(11)$ & $3,527(1)$ \\
\hline & 5 & 5,405 & 25 & $18,19(6)$ & $1,0810(4)$ & $1627,09(10)$ & $3,494(1)$ \\
\hline 2 & 5 & 5,378 & 35 & $14,59(2)$ & $1,0755(4)$ & $1633,20(9)$ & $3,486(1)$ \\
\hline 0 & 7 & 7,839 & 15 & $23,82(17)$ & $1,1198(4)$ & $1676,77(12)$ & $3,176(1)$ \\
\hline & 7 & 7,802 & 25 & $18,51(7)$ & $1,1145(4)$ & $1680,25(12)$ & $3,178(1)$ \\
\hline & 7 & 7,765 & 35 & $15,01(4)$ & $1,1092(4)$ & $1680,89(12)$ & $3,191(1)$ \\
\hline 2 & 8 & 9,106 & 15 & $24,34(8)$ & $1,1382(4)$ & $1706,51(15)$ & $3,017(1)$ \\
\hline 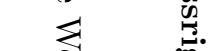 & 8 & 9,069 & 25 & $19,13(7)$ & $1,1336(4)$ & $1706,33(14)$ & $3,030(1)$ \\
\hline & 8 & 9,021 & 35 & $15,62(4)$ & $1,1276(4)$ & $1704,12(13)$ & $3,054(1)$ \\
\hline
\end{tabular}




\section{Kohlenhydrate}

Der historische Begriff „Kohlenhydrat“ umfasste ursprünglich eine Stoffklasse, die die Elemente Kohlenstoff, Wasserstoff und Sauerstoff im Verhältnis 1:2:1 enthält („Hydrat der Kohle“). Der Name ist auch heute noch gebräuchlich, obwohl keineswegs alle in der Natur vorkommenden Zucker der Summenformel $\mathrm{C}_{n}\left(\mathrm{H}_{2} \mathrm{O}\right)_{n}$ im Fall der Monosaccharide bzw. $\mathrm{C}_{n}\left(\mathrm{H}_{2} \mathrm{O}\right)_{m}$ für Polysaccharide genügen. Auf der anderen Seite gibt es auch Moleküle, wie die Milchsäure, die zwar obiger Definition entsprechen, aber aufgrund ihrer Charakteristika nicht den Zuckern zugeordnet werden können. Man teilt die Kohlenhydrate entsprechend ihrer chemischen und physikalischen Eigenschaften i. a. in drei Hauptgruppen ein:

- M onosaccharide:

Die Monosaccharide oder einfache Zucker bilden die Grundbausteine für die folgenden Gruppen. Sie werden in Abschnitt (6.1) ausführlicher beschrieben. Ihre bekanntesten Vertreter sind der Traubenzucker (Glucose) und der Fruchtzucker (Fructose).

- Oligosaccharide:

Bei diesen Zuckern sind 2 bis 6 Monosaccharide unter Wasserabspaltung acetalartig aneinander gebunden. Handelt es sich um eine Verbindung von zwei Monosacchariden so spricht man von einem Disaccharid. Diese werden im Kapitel 6.2 vorgestellt. Entsprechend spricht man von Trisacchariden bei einer Verbindung aus drei einfachen Zuckern, sowie von Tetra- Penta- und Hexasacchariden bei den höheren Formen.

- Polysaccharide:

Erhöht man die Monomeranzahl der Kohlenhydratketten weiter, so gelangt man zu den Polysacchariden. Die Eigenschaften dieser hochmolekularen Polymere unterscheiden sich im Allgemeinen deutlich von denen der Mono- und Oligosaccharide. Die Polymerketten können dabei linear wie auch verzweigt auftreten. Wichtige Vertreter sind Stärke, Glykogen und Cellulose.

\subsection{Monosaccharide}

Einfache Zucker können als Oxidationsprodukte mehrwertiger Alkohole betrachtet werden. Wird eine primäre Hydroxy-Gruppe oxidiert so erhält man ein PolyhydroxyAldehyd (mit der Aldehydgruppe CHO). Diese Zucker werden Aldosen genannt. Bei einer sekundären Hydroxy-Gruppe entsteht ein Polyhydroxy-Keton (mit der Ketogruppe CO), eine Ketose (siehe Abbildung 6.1). Bei nahezu allen Zuckern liegt dabei eine unverzweigte Kohlenstoffkette vor, deren Länge zunächst nicht beschränkt ist. Natürlich vorkommende Zucker haben meist eine Kettenlänge von fünf oder sechs Kohlenstoffatomen. Man kennzeichnet Aldosen mit der Namensendung -ose bzw. 

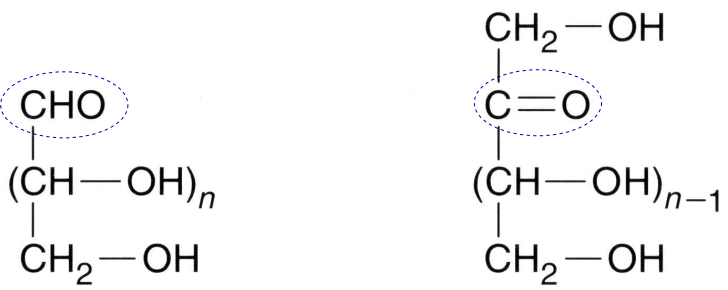

\begin{abstract}
Abbildung 6.1: Aldosen und Ketosen. Auf der linken Seite sind Aldehyd-Zucker (Aldosen) und rechts Keto-Zucker (Ketosen) dargestellt. Die namensgebende funktionelle Gruppe ist eingekreist.
\end{abstract}

Ketosen mit -ulose und der Wortstamm gibt die Zahl der Kohlenstoffatomen der Kette an. Eine Aldose mit drei C-Atomen ist somit eine Triose, bei vier, fünf und sechs C-Atomen spricht man von Tetrose, Pentose und Hexose. Bei Ketosen entsprechend von Tetrulose, Pentulose und Hexulose. Eine „Triulose“ existiert nicht, da ein Monosaccharid zumindest über ein asymmetrisches $\mathrm{C}$-Atom verfügen muss, und das 1,3-Dihydroxyaceton ein solches nicht besitzt.

\title{
6.1.1 Konstitution und Konfiguration von Monosacchariden
}

Eine der interessantesten Eigenschaften der Monosaccharide ist ihre Fähigkeit, bei gleicher Summenformel eine Fülle von Isomeren mit z.T. deutlich unterschiedlichen Eigenschaften zu bilden. Ist bereits - bei gegebener Summenformel - die Verknüpfung der Atome durch kovalente Bindungen unterschiedlich, so spricht man von Konstitutionsisomeren. Ein Beispiel für eine solche Isomerie sind Fructose (Fruchtzucker) und Glucose (Traubenzucker), die beide die Summenformel $\mathrm{C}_{6} \mathrm{H}_{12} \mathrm{O}_{6}$ besitzen (vgl. Abb. 6.2). Ist im nächsten Schritt zwar die Konstitution der Moleküle identisch, allerdings die räumliche Anordnung der einzelnen Atome - die Konfiguration - verschieden, so spricht man von Stereoisomerie. Liegen die Moleküle hierbei wie Bild und Spiegelbild vor, so sind es Enantiomere, ansonsten Diastereomere. Aldohexosen besitzen vier asymmetrische $\mathrm{C}$-Atome und bildet daher $2^{4}$ Stereoisomere in 8 Enantiomerenpaaren. Im Falle der Ketohexosen sind es nur drei asymmetrische CAtome, somit existieren $2^{3}$ Stereoisomere in 4 Enantiomerenpaaren. Da Enantiomere auch im Fall der Monosaccharide nahezu identische Eigenschaften besitzen, tragen sie den gleichen (Trivial-) Namen und werden durch ein vorangestelltes D- oder Lunterschieden. Heute wird die Zugehörigkeit eines Zuckers zur D- oder L-Familie ausschließlich aus der Fischer-Projektion gewonnen und bezieht sich dabei auf das Glycerinaldehyd. Zeigt die Hydroxy-Gruppe des am weitesten von der Keto- oder Aldehydgruppe entfernten asymmetrischen Kohlenstoffs in dieser Projektion nach links, so gehört der Zucker zur L-Serie, nach rechts entsprechend zur D-Serie (vgl. Abbildungen 6.2 und 6.4). Physikalisch lassen sich die beiden Enantiomere relativ einfach durch die unterschiedliche Drehung der Polarisationsebene bei Einstrahlung von linear polarisiertem Licht in eine wässrige Lösung unterscheiden. Beide drehen - bei gleicher Konzentration, Schichtdicke, Temperatur und Wellenlänge - die Polarisationsebene des Lichts um den gleichen Betrag, allerdings in entgegengesetzte 
<smiles>O=C[Z](O)(O)C(O)(O)[13CH](O)[13CH](O)O</smiles>

D-Glucose<smiles>O=[Z](O)C(O)(C(O)(O)CO)C(O)(O)CO</smiles>

D-Fructose

\begin{abstract}
Abbildung 6.2: Ein Beispiel für Konstitutionsisomerie bei Monosacchariden. Sowohl der Aldehyd-Zucker Glucose wie der Keto-Zucker Fructose besitzen die Summenformel $\mathrm{C}_{6} \mathrm{H}_{12} \mathrm{O}_{6}$. Die Hydroxy-Gruppe des am weitesten von der AldehydGruppe entfernten asymmetrischen Kohlenstoffatoms $\left(\mathrm{C}_{5}\right)$ bestimmt durch die Ausrichtung nach rechts die Zugehörigkeit zur D-Reihe. Das sechste Kohlenstoffatom ist mit zwei Wasserstoffen verbunden und daher nicht asymmetrisch.
\end{abstract}

Richtung. Zu beachten ist, dass die Zugehörigkeit zur D- oder L-Familie keine pauschale Auskunft über den Drehsinn gibt, daher werden meist beide Informationen dem Namen vorangestellt, z.B. D(+)-Glucose, D(-)-Fructose.

Die Unterschiede in den Eigenschaften der Diastereomere sind hingegen bereits so beträchtlich, dass die Zucker mit verschiedene Namen bezeichnet werden. Als Beispiel sind in Abbildung 6.3 die D-Glucose und D-Galactose in Ringform (siehe Abschnitt 6.1.2) dargestellt, bei denen lediglich die Stellung der Hydroxy-Gruppe am vierten Kohlenstoffatom variiert. Trotz dieses vermeindlich nur geringen Unterschieds zeigen sie deutlich abweichende Ultraschall-Absorptionsspektren (vgl. Abbildungen A.1 und A.3). Man kann die verschiedenen Monosaccharide aufgrund der

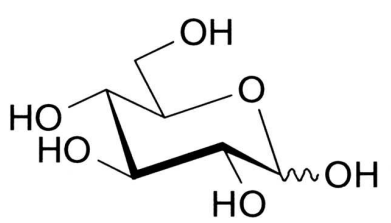

D-Glucose

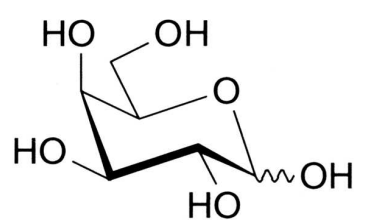

D - und L-Galactose

Abbildung 6.3: Darstellung von Glucose und Galactose in Ringform [36].

Länge der Kohlenstoffkette und der Isomerie in einen sogenannten MonosaccharidStammbaum einordnen, wie ihn die Abbildungen 6.4 und 6.5 zeigen. 


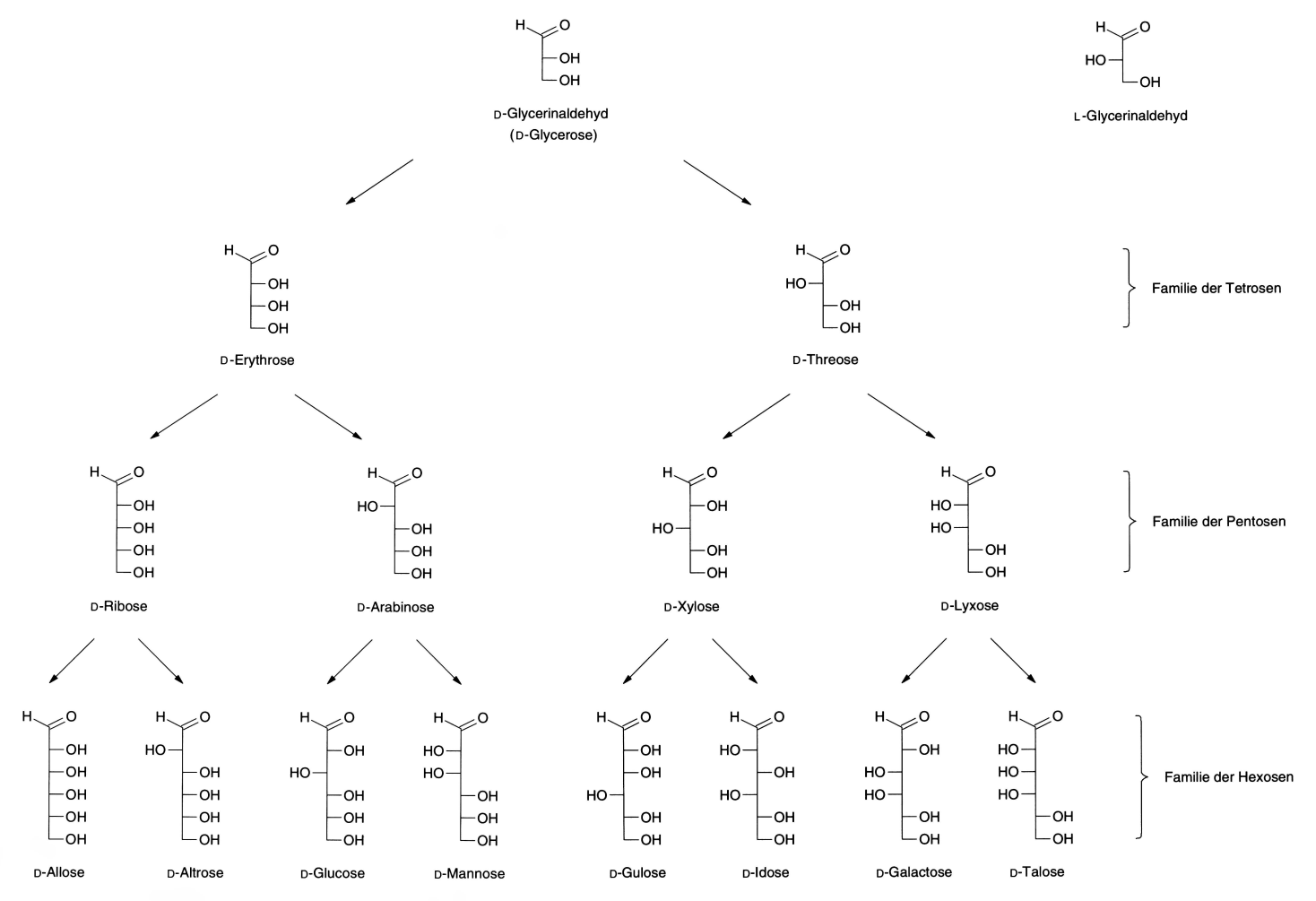

Abbildung 6.4: Monosaccharid-Stammbaum für die D-Reihe der Aldosen. Die einzelnen Monosaccharide sind in der Fischer-Projektion dargestellt [36].

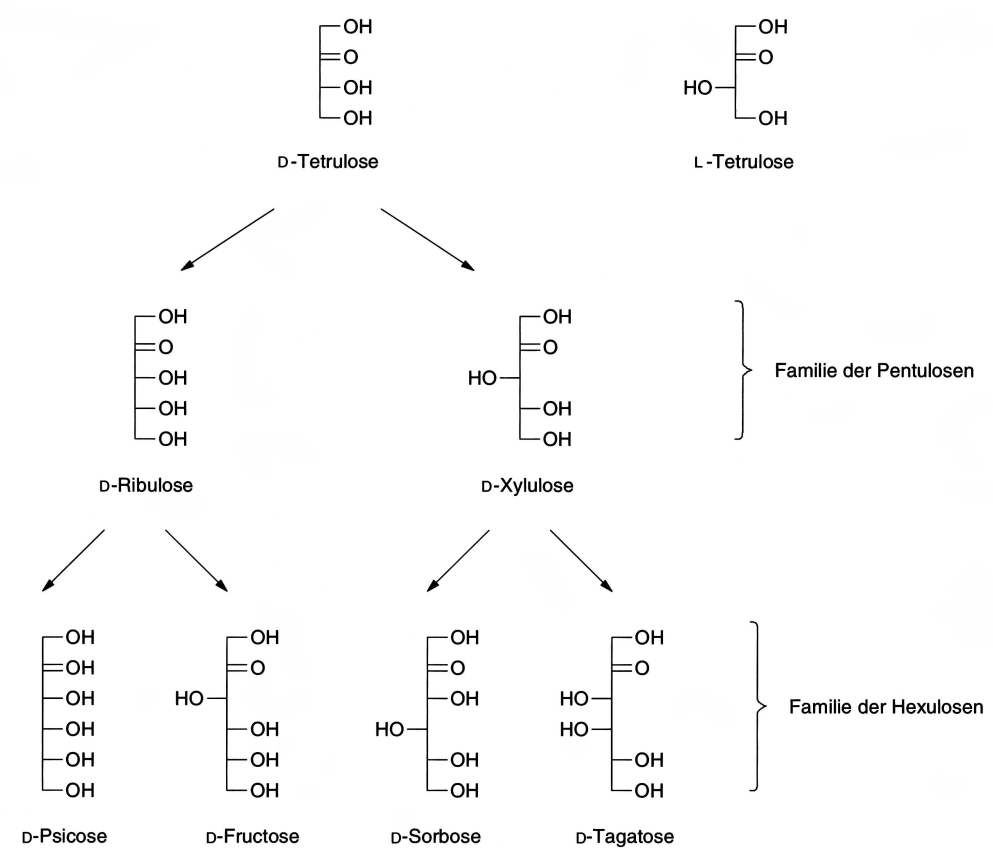

Abbildung 6.5: Monosaccharid-Stammbaum der D-Reihe der Ketosen in der Fischer-Projektion [36]. 


\title{
6.1.2 Ring-Ketten-Tautomerie
}

Allen bisherigen Betrachtungen über Monosaccharide lag die offenkettige Form zugrunde. Diese liegt in wässrigen Lösungen jedoch nur in sehr geringer Konzentration vor. Energetisch wesentlich günstiger ist bei den Pentosen und Hexosen (bzw. Pentulosen und Hexulosen) eine Ringbildung über eine intramolekulare Halbacetalbindung, wie sie von Abbildung 6.6 für den Aldehyd-Zucker Glucose gezeigt wird. Der Unterschied in der freien Energie zwischen acyclischer und pyranose Form beträgt hier 29,4 kJ/mol [36]. Bei den Aldehydzuckern ist an der Halbacetalbindung
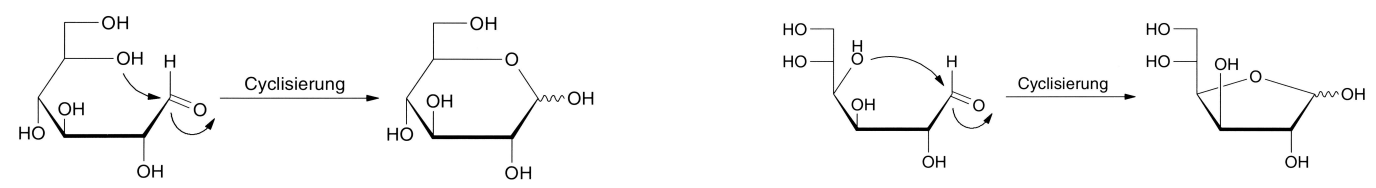

Abbildung 6.6: Ringschluss der Glucose zu Glucopyranose (links) und Glucofuranose (rechts) [36].

stets das $\mathrm{C}_{1}-$ Atom, die Aldehydgruppe, beteiligt. Erfolgt der Ringschluss über die Hydroxy-Gruppe am $\mathrm{C}_{5}$-Atom, so entsteht ein 6-gliedriger heterocyclischer Ring der dem Pyran ähnelt, eine Pyranose. Das einzelne im Ring enthaltene Sauerstoffatom bewirkt dabei eine Symmetriebrechung im Vergleich zum Cyclohexan. Durch den Ringschluss ergibt sich ein weiteres asymmetrisches Kohlenstoffatom $\left(\mathrm{C}_{1}\right)$, das als anomeres $\mathrm{C}$-Atom bezeichnet wird. Die zugehörige Hydroxy-Gruppe unterscheidet sich in ihren Eigenschaften deutlich von den übrigen und wird daher mit einem eigenen Namen, Lactol-Gruppe, versehen. Handelt es sich um eine Pentose, so sind alle $\mathrm{C}$-Atome im Ring integriert, bei einer Hexose ist das $\mathrm{C}_{6}-$ Atom exocyclisch angeordnet. Der Ring selbst ist aufgrund der nur leicht veränderten Tetraederbindungswinkel nicht eben, sondern kann prinzipiell, wie in Abbildung 6.7 dargestellt, eine Reihe von Konformationen annehmen. Welche davon bei einem speziellen Zucker tatsächlich energetisch günstig sind und wie die „Energielandschaft" aussieht hängt stark von der Anordnung der Hydroxy- und, falls vorhanden, exocyclischen Seitengruppen ab. Bei der häufigsten Sessel-Konformation können zwei Stellungen der
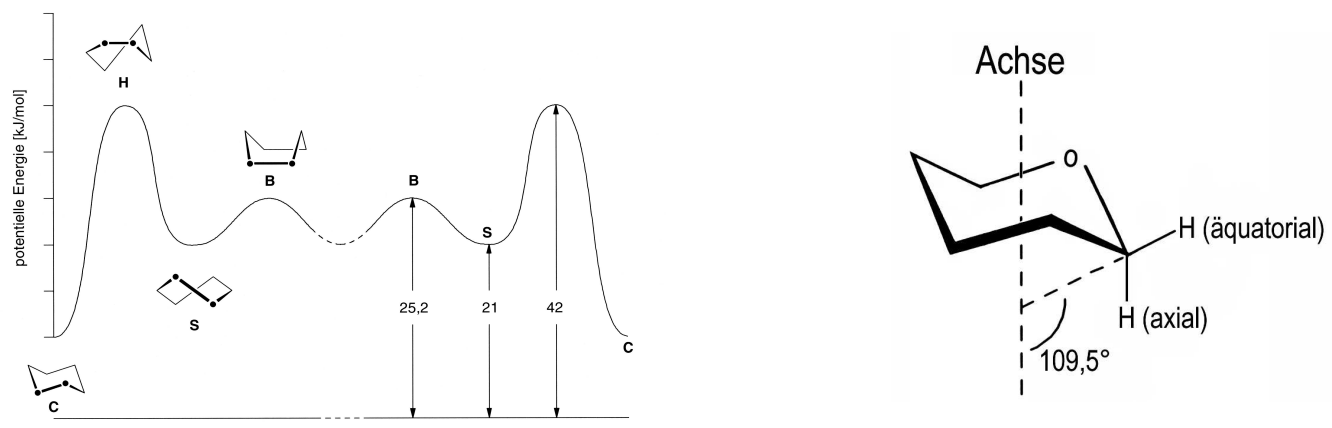

\begin{abstract}
Abbildung 6.7: Schema der Ringkonformationen und Energiebarrieren bei einem sechsgliedrigen Ringmolekül. C (Chair) Sesselkonformation; H (Half chair) Halbsessel; S (Skew) verdrehte Wanne; B (Boat) Wanne. Rechte Seite: Axiale und äquatoriale Ausrichtung der Seitengruppen [36].
\end{abstract}

Seitengruppen unterschieden werden: Entweder sie verlaufen normal zur gedachten Ringebene - man spricht dann von einer axialen Richtung - oder sie sind bei der 
äquatorialen Richtung leicht aus der Ringebene geneigt. Der Ringschluss, und damit verbunden das zusätzliche asymmetrische C-Atom, führt zu zwei zusätzlichen Diastereomeren. Ist die Lactol-Gruppe in der Darstellung als ${ }^{4} \mathrm{C}_{1}$-Sessel äquatorial angeordnet, so erhält man die $\beta$-Konfiguration, bei axialer die $\alpha$-Konfiguration. Ist am

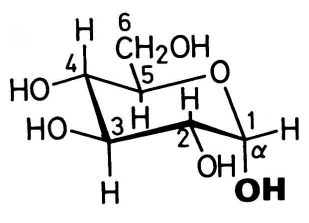

$\alpha$-D-Glucose

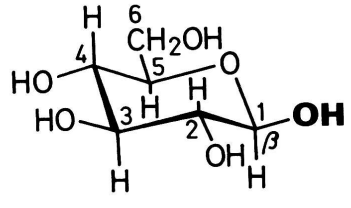

$\beta$-D-Glucose

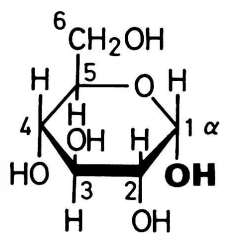

$\alpha$-D-Glucose

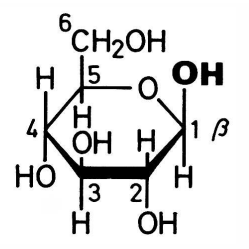

$\beta$-D-Glucose

Abbildung 6.8: $\alpha$ - und $\beta$-Konfiguration der Glucose in der Darstellung als ${ }^{4} \mathrm{C}_{1}$-Sessel und in der vereinfachten Harworth-Projektion [39].

Ringschluss hingegen das $\mathrm{C}_{4}-$ Atom beteiligt, so entsteht ein fünfgliedriger Ring. Der Zucker wird dann - namentlich angelehnt an das Furan - als Furanose bezeichnet. Bei Pentosen gibt es mit dem $\mathrm{C}_{5}$-Atom eine exocyclische Hydroxymethyl-Gruppe, bei den Hexosen eine Dihydroxyethyl-Gruppe. In wässrigen Lösungen treten die Furanosen der Aldehydzucker nur in geringen Konzentrationen von wenigen Prozent auf.

Auch bei den Ketozuckern sind die Ringformen energetisch bevorzugt. Da der Ringschluss über die Keto-Gruppe am $\mathrm{C}_{2}$-Atom erfolgt, liegt das $\mathrm{C}_{1}-$ Atom stets als exocyclische Gruppe vor. Daher können die Pentulosen nur fünfgliedrige Furanosen bilden. Hexulosen hingegen existieren sowohl als fünf wie auch sechsgliedrige Ringe. Bei den Pyranosen liegt wie auch im Falle der Hexosen eine exocyclische Gruppe vor, allerdings ist es das $\mathrm{C}_{1}$ und nicht das $\mathrm{C}_{6}-$ Atom. Bei den Furanosen gibt es hingegen zwei exocyclische Hydroxymethyl-Gruppen, das $\mathrm{C}_{1}$ und das $\mathrm{C}_{6}$-Atom.

Bei den Ketozuckern stellt die Fructose eine Besonderheit dar, da bei ihr in wässrigen Lösungen ca. 28\% der Moleküle als Furanose vorliegen. Die angesprochene Symmetriebrechung ermöglicht gegenüber dem Cyclohexan zusätzliche Konformationen, die sich nicht durch Drehungen ineinander überführen lassen. Die aus Sicht der Ultraschallspektroskopie interessanteste ist die Existenz zweier möglicher Sesselformen bei den Pyranoseringen. Betrachtet man bei den Aldehydzuckern den Ring so, dass das Sauerstoffatom hinter der Bildebene rechts angeordnet ist, dann bildet die Ebene aus Sauerstoff, $\mathrm{C}_{2}, \mathrm{C}_{3}$ und $\mathrm{C}_{5}$-Atom die „Sitzfläche“ des Sessels. Für „Lehne" und „Fußteil" gibt es zwei Möglichkeiten: Entweder ist das $\mathrm{C}_{1}-$ Atom unterhalb und das $\mathrm{C}_{4}$ entsprechend oberhalb dieser Ebene angeordnet oder es verhält sich umgekehrt. Man bezeichnet die Formen als ${ }^{4} \mathrm{C}_{1}$ und ${ }^{1} \mathrm{C}_{4}-$ Sessel. Der obere Index gibt jeweils an, welches Kohlenstoffatom über der Ebene angeordnet ist. Abbildung 6.9 zeigt ein solches Gleichgewicht. Bei den Ketozuckern ist das $\mathrm{C}_{1}-$ Atom exocyclisch angeordnet; hier liegt daher ein ${ }^{2} \mathrm{C}_{5} \rightleftharpoons{ }^{5} \mathrm{C}_{2}$ Sessel-Sessel-Gleichgewicht vor (siehe Abb. 6.10). Man beachte, dass bei der Beurteilung, ob es sich um die $\alpha-$ oder $\beta$-Konfiguration handelt, d.h. ob die anomere Gruppe axial oder äquatorial ausgerichtet ist, die zugrundeliegende Sesselform berücksichtigt werden muss. Die Halbacetal-Bindung, die den Ringschluss bildet, ist weniger stabil als die anderen kovalenten Bindungen im Ring. Unter Normalbedingungen reicht die thermische 


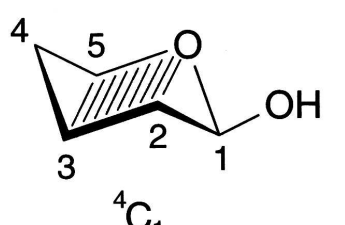

${ }^{4} \mathrm{C}_{1}$

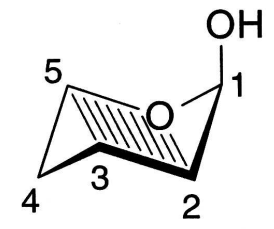

${ }^{1} \mathrm{C}_{4}$

Abbildung 6.9: Sessel-Sessel Gleichgewicht bei Pyranosen. Links: ${ }^{4} \mathrm{C}_{1}-\mathrm{Sessel}$; Rechts: ${ }^{1} \mathrm{C}_{4}-$ Sessel [36].

Energie aus, um diese Bindung aufbrechen zu lassen, und es entsteht die acyclische Form. Beim erneuten Ringschluss kann es vorkommen, dass nicht wieder die gleiche Ringform gebildet wird, sondern aus einer $\alpha$ - eine $\beta$-Form oder umgekehrt. Auch die Bildung eines Furanose- aus einem Pyranosering bzw. die Umkehrung ist möglich. Bei den Hexosen, Pentosen und Hexulosen in Lösung stehen also über die offenkettige Form die vier möglichen Ringe im Gleichgewicht, während als Feststoff meist nur ein Ringtyp vorliegt. Dieser Vorgang wird als Mutarotation bezeichnet. Die Einstellung des Gleichgewichts nach Lösen des Feststoffes in Wasser dauert unter Normalbedingungen Minuten bis Stunden. Die Abbildung 6.10 zeigt als Beispiel das Mutarotationsgleichgewicht und das Sessel-Sessel-Gleichgewicht für Fructose. Hier ist $\mathrm{zu}$ beachten, dass beim Ketozucker Fructose das $\mathrm{C}_{1}$-Atom exocyclisch angeordnet ist, die beiden Sesselformen also ${ }^{5} \mathrm{C}_{2}$ und ${ }^{2} \mathrm{C}_{5}$ lauten.

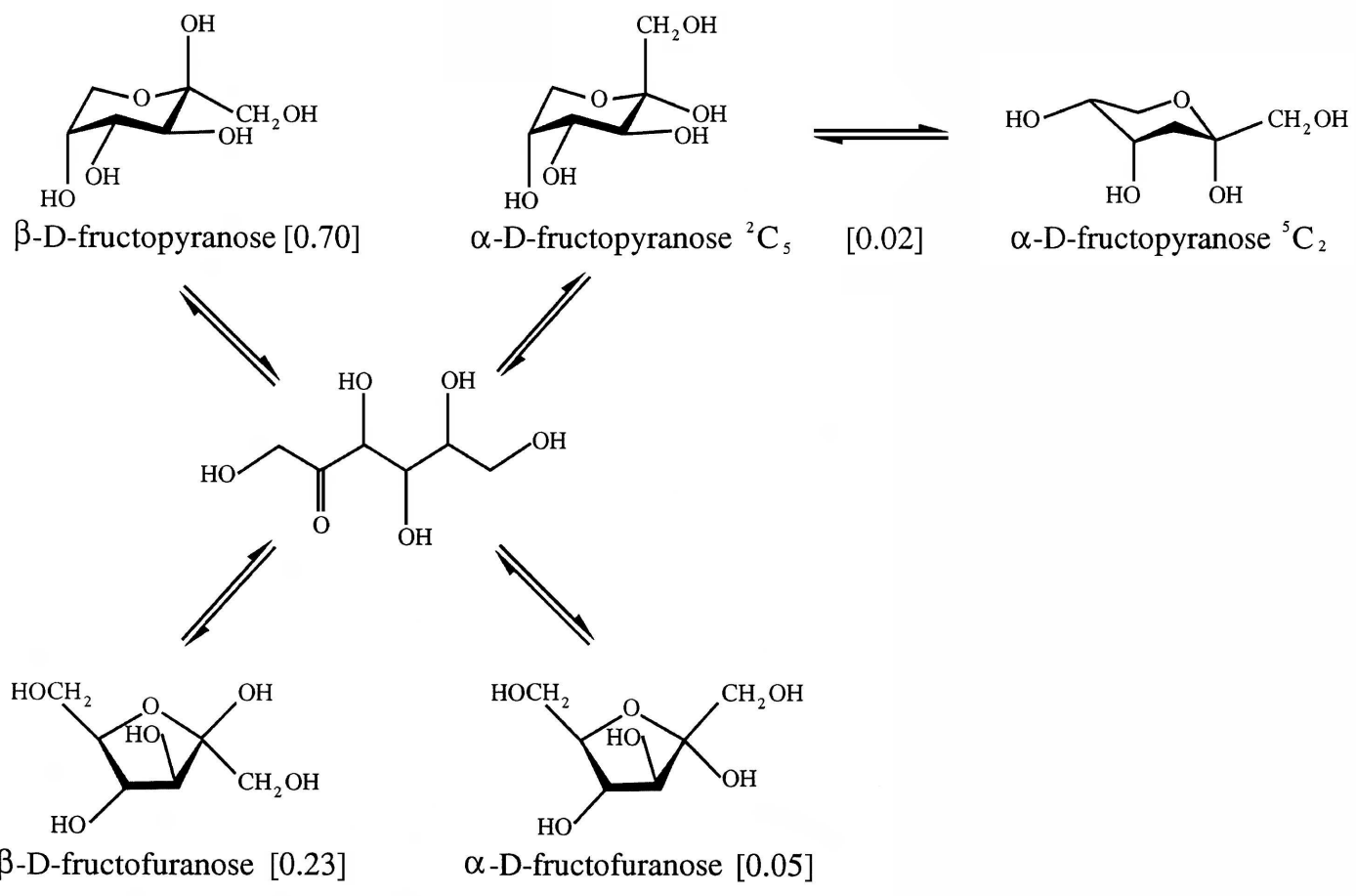

Abbildung 6.10: Mutarotations und Sessel-Sessel Gleichgewicht von Fructose. In Klammern ist der Anteil der jeweiligen Spezies angegeben. 


\subsection{Disaccharide}

Disaccharide sind für die Untersuchung aller höher molekularen Oligo- und Polysaccharide interessant, da mit ihnen die kleinsten Einheiten vorliegen, bei denen zwei Monosaccharide über eine Glycosid-Bindung miteinander verbunden sind. Genauer handelt es sich bei den Sacchariden um eine Sauerstoff-Glycosid-Bindung, da Stickstoff-Glycosid-Bindungen für die hier betrachteten Moleküle keine Rolle spielen, wird diese Unterscheidung im Folgenden nicht mehr vorgenommen. Disaccharide bilden also ein möglichst einfaches Modell für die Untersuchung der Eigenschaften dieser für Kohlenstoffketten wichtigen Bindungsform. Die Verknüpfung der Monosaccharide erfolgt stets über die anomere Gruppe eines der Monomere, die unter Wasserabspaltung mit einer Hydroxy-Gruppe des zweiten Ringes reagiert. Bei den Hexosen gibt es hier fünf Möglichkeiten zur Auswahl. Da sich die anomere Hydroxy-Gruppe von den restlichen, alkoholischen deutlich in ihren Eigenschaften unterscheidet, muss auch bei den Disacchariden differenziert werden, ob an der Bindung die zweite anomere Gruppe beteiligt ist. Chemisch gesehen unterscheiden sich die Varianten in ihren reduzierenden Eigenschaften, interessanter für diese Arbeit ist dabei, dass bei einem Disaccharid ohne freie anomere Gruppe keine Mutarotation mehr auftritt. Außerdem ist bei dieser Form eine weitere gleichartige Verknüpfung zu Polysaccharidketten nicht möglich. Ist hingegen eine der alkoholischen Hydroxy-Gruppen an der Glycosid-Bindung beteiligt, so können aus gleichartigen Monomereinheiten lange Saccharid-Polymere entstehen (vgl. Abschnitt 6.2.1).

Im Folgenden werden anhand der für diese Arbeit untersuchten Disaccharide charakteristische Eigenschaften und die Art der Verknüpfung der Monosaccharide vorgestellt.

\subsubsection{Definition der glycosidischen Bindungswinkel $\phi, \psi$ und $\omega$}

Bei der Bindung zweier Monosaccharide zu einem Disaccharid gibt es zwei sterisch unterschiedliche Arten der Brücke zwischen den Ringen. Erfolgt die Bindung bei Pyranosen über eine Hydroxy-Gruppe an den Kohlenstoffatomen eins bis vier des zweiten Monosaccharides, so liegt eine Sauerstoffbrücke mit zwei nicht fixierten Einfachbindungen vor, um die die einzelnen Ringe ihre gegenseitige Ausrichtung variieren können. Die Winkel der Rotation um diese Bindungen werden mit $\phi$ und $\psi$ bezeichnet, wobei $\phi$ die Rotation um die $\mathrm{C}_{1}-\mathrm{O}_{1}$ Bindung beschreibt, $\psi$ diejenige um $\mathrm{O}_{1}-\mathrm{C}_{i}(\mathrm{i}=2,3,4)$. Abbildung 6.11 zeigt diese am Beispiel der Maltose. Zusätzlich ist noch zu unterscheiden, ob die anomere Gruppe des ersten Monosaccharides in $\alpha$ - oder $\beta$-Form vorliegt. So ist z.B. die Amylose - ein Bestandteil der Stärke - eine Kette aus $\alpha(1,4)$-glycosidisch verknüpften Glucopyranosen, während diese in der Cellulose $\beta(1,4)$-glycosidisch verkettet sind. Nur die andere Stellung am ersten Kohlenstoffatom führt hier zu den deutlich verschiedenen physikalischen Eigenschaften dieser natürlichen Kohlenhydrate. Erfolgt die Verknüpfung der Ringe hingegen über die Hydroxy-Gruppe des sechsten Kohlenstoffatoms, der exocyclischen Gruppe, so entsteht eine Sauerstoff-Kohlenstoff-Brücke mit drei nichtfixierten Einfachbindungen. Der Winkel der Rotation um die $\mathrm{C}_{6}-\mathrm{C}_{5}$ Bindung wird mit $\omega$ bezeichnet $\left(\mathrm{O}_{6}{ }^{-}\right.$ $\mathrm{C}_{6}-\mathrm{C}_{5}-\mathrm{O}_{5}$ Drehwinkel). Hier muss die $\alpha$ - oder $\beta$-Form des ersten Monosaccharides ebenfalls berücksichtigt werden. Ein Beispiel für diese Art der Verknüpfung ist die Melibiose (vgl. Abb. 6.15). Außerdem tritt sie bei verzweigten Kohlenstoffketten an 


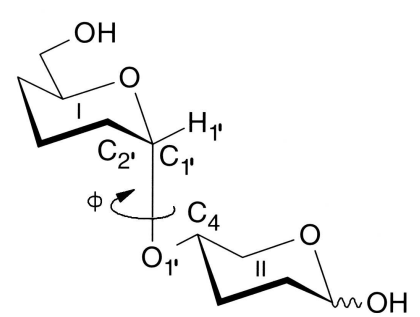

Blickrichtung: Projektion $\mathrm{C}_{1^{1}} \rightarrow \mathrm{O}_{1^{\prime}}$

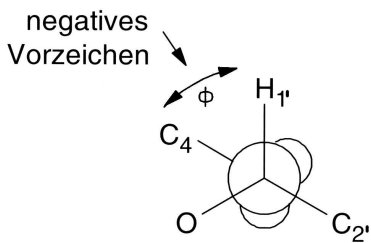

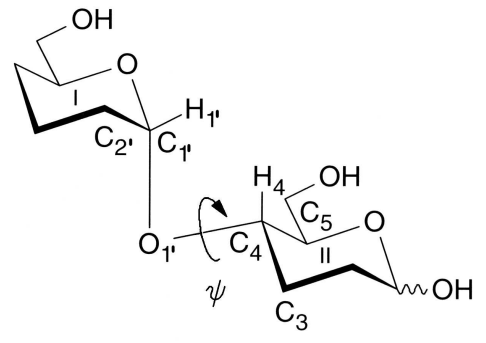

Blickrichtung: Projektion $\mathrm{C}_{4} \rightarrow \mathrm{O}_{1}$

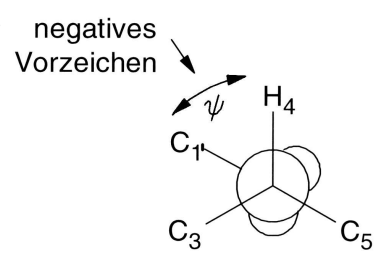

Abbildung 6.11: Definition der glycosidischen Bindungswinkel $\phi$ und $\psi$ am Beispiel der Maltose [36].

der Verküpfungstelle auf. So enthält Amylopektin - mit 80\% der Hauptbestandteil der Stärke - $\alpha(1,6)$-glycosidische Verzweigungsstellen [38].

Häufig wird bereits die Rotation der exocyclischen Gruppe allein, ohne ein weiteres gebundenes Monosaccharid als einfachstes Modellsystem für die Untersuchung von $\omega$ betrachtet. Daher sind in Abbildung 6.12 die drei möglichen Rotamereinstellungen für Methyl- $\alpha-$ D-Glucopyranosid dargestellt. Allerdings ist hier die tg-
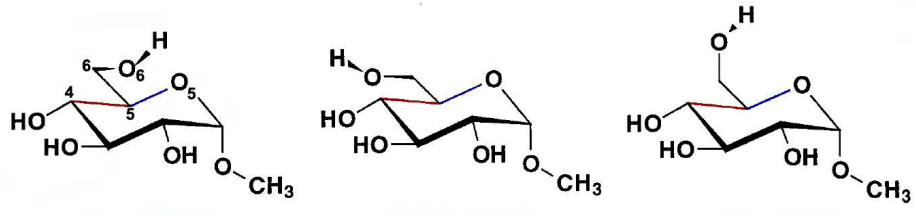

gt $\left(\omega \sim 60^{\circ}\right)$

$\operatorname{tg}\left(\omega \sim 180^{\circ}\right)$

$\operatorname{gg}\left(\omega \sim 300^{\circ}\right)$

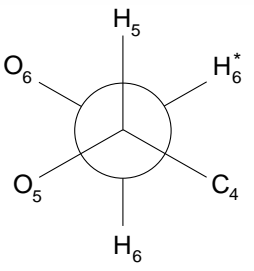

gt

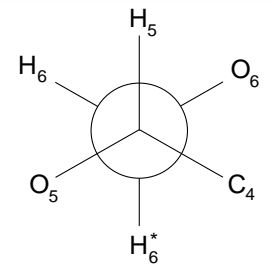

$\operatorname{tg}$<smiles>[2H]C1C(C)C2(C)C([O])C([Hg])C1(C)C2(C)C</smiles>

gg

Abbildung 6.12: Abbildung der gt, tg und gg-Konfigurationen bei Rotation um $\omega$ für Methyl $\alpha-\mathrm{D}-$ Glucopyranosid [64]. Unten: Darstellung in der NewmanProjektion. 
Konformation aufgrund der ungünstigen abstoßenden Wechselwirkung zwischen den Hydroxy-Gruppen am sechsten und vierten Kohlenstoffatom nur gering bevölkert. Ändert man die Position der Hydroxy-Gruppe am $\mathrm{C}_{4}$-Atom von äquatorial auf axial, geht mithin vom Methyl- $\alpha-$ D-Glucopyranosid zum Methyl- $\alpha-$ D-Galactopyranosid, so ist entsprechend die gg-Konformation energetisch ungünstig. Die am ersten Sauerstoffatom methylierte Variante der Glucose bietet für Untersuchungen zum einen den Vorteil, dass die besonderen Eigenschaften der anomeren Gruppe („exo anomerer Effekt") nicht berücksichtigt zu werden brauchen, zum anderen sind bei diesem Molekül auch $\phi$ und $\psi$ bereits vorhanden. Mithin kann es als einfachstes, wenn auch unvollständiges Modell für die Untersuchung von Polysaccharid-Ketten herangezogen werden.

Auch im Falle von $\phi$ und $\psi$ wird die freie Rotation nicht durch eine Fixierung der Einfachbindungen, wohl aber durch sterische Behinderungen der Monosaccharideinheiten sowie intramolekulare Wechselwirkungen und Lösungsmitteleinflüsse behindert. Abbildung 6.13 enthält eine zweidimensionale Darstellung der Wechselwirkungsenergien bei Rotation um die Bindungswinkel $\phi$ und $\psi$ für charakteristische Formen der Glycosid-Bindung am Beispiel von vier Disacchariden. Man kann erkennen, dass energetisch günstigen Konformationen relativ enge Grenzen gesetzt sind, nach Brant [54] umfasst der zugängliche Bereich für Energien bis 5 RT oberhalb des Minimums gerade 2,5\% des Konformationsraumes. Für Maltose liegt $\phi$ hierbei im Bereich von ca. $-20^{\circ}$, während der Winkel $\psi$ zwei stabile Bereiche bei ca. $0^{\circ}$ („syn“) und $180^{\circ}$ (, ,anti“) zeigt. In Abbildung 6.11 ist die syn-Konformation dargestellt. Molekulardynamikrechnungen von Frank [66] sowie Meyer und Ott [63] zeigen das globale Minimum in der syn-Konformation und ein lokales in der anti-Konformation, wobei das globale Minimum nach Frank nochmals in vier Cluster eingeteilt werden kann. Für die untersuchten Disaccharide, die im folgenden Abschnitt vorgestellt werden, erhält man die folgenden Bindungstypen:

\begin{tabular}{|l|l|l|l|l|l|}
\hline Disaccharid & \multicolumn{3}{|c|}{ Monosaccharid I } & \multicolumn{3}{c|}{ Monosaccharid II } \\
& Name & Stellung & Stellung & C-Atom & Name \\
\hline Maltose & Glucopyranose & axial & äquat. & $\mathrm{C}_{4}$ & Glucopyranose \\
Trehalose & Glucopyranose & axial & axial & $\mathrm{C}_{1}$ & Glucopyranose \\
Lactose & Galactopyranose & äquat. & äquat. & $\mathrm{C}_{4}$ & Glucopyranose \\
Sucrose & Glucopyranose & axial & - & $\mathrm{C}_{2}$ & Fructofuranose \\
Melibiose & Galactopyranose & axial & - & $\mathrm{C}_{6}$ & Glucopyranose \\
\hline
\end{tabular}

Tabelle 6.1: Charakterisierung der Glycosid-Bindung der gemessenen Disaccharide. Unter Stellung ist die Ausrichtung der an der Brücke beteiligten Bindung in Bezug auf die Ringebene angegeben. 

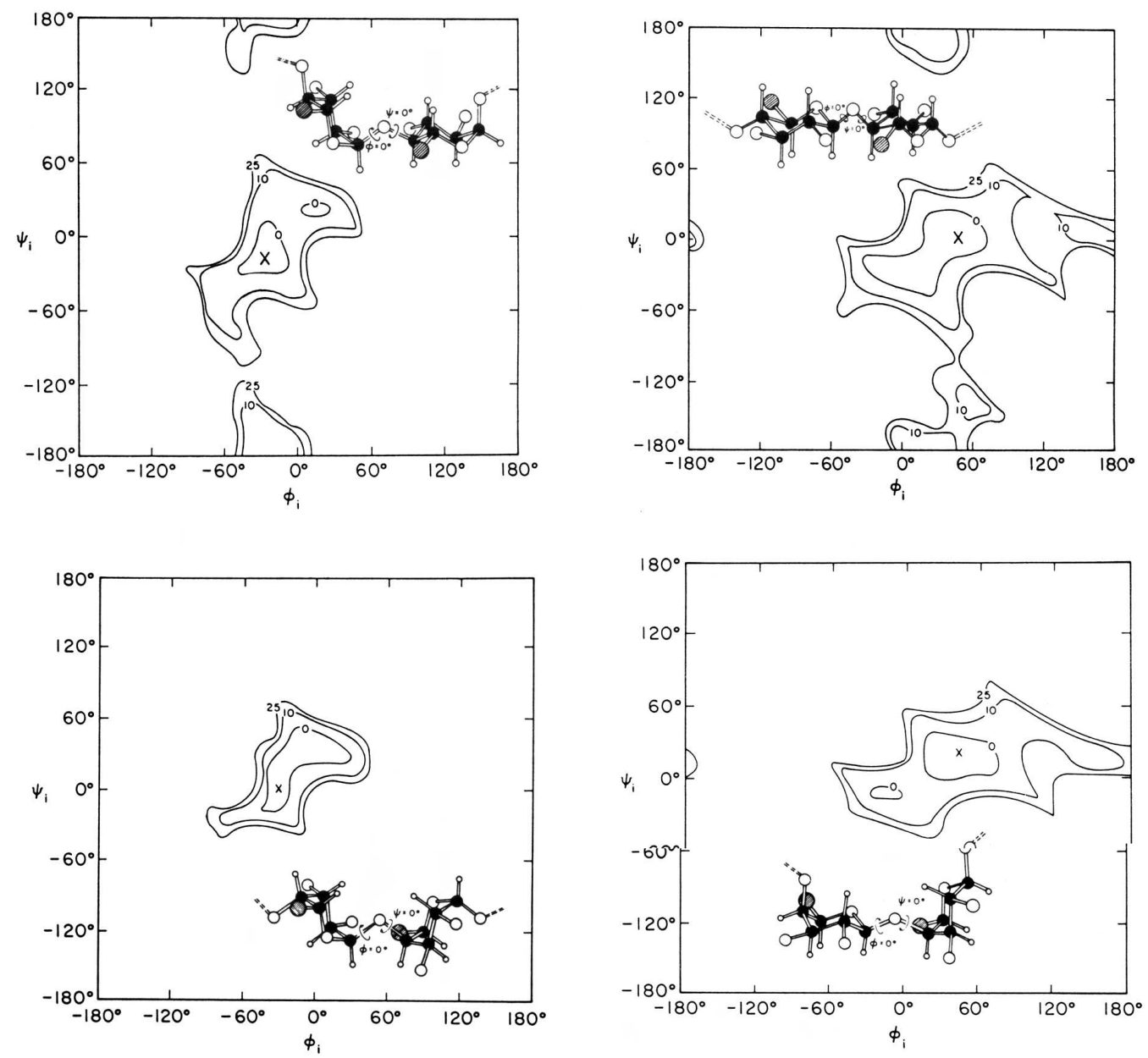

Abbildung 6.13: Einstellung der glycosidischen Bindungswinkel $\phi$ und $\psi$. Konturdiagramme der Konformationsenergie am Beispiel der Maltose (axial-äquatorial, oben links), Cellobiose (äquatorial-äquatorial, oben rechts), $\alpha$-Galactan (axialaxial, unten links) und $\beta$-Galactan (äquatorial-axial, unten rechts). Die Zahlen geben die Energie in $\mathrm{kcal} / \mathrm{mol}$ an, die Konformation niedrigster Energie ist mit $\times$ gekennzeichnet [54]. 


\subsubsection{Maltose}

Im Falle der Maltose sind zwei D-Glucose Moleküle zu einem Disaccharid verbunden. Die Verbindung der Monosaccharide erfolgt vom anomeren Kohlenstoffatom der einen Glucose über eine Sauerstoffbrücke zum vierten C-Atom der zweiten. Damit bleibt eine anomere Gruppe in ihrer Funktion erhalten, die Maltose ist reduzierend, zeigt Mutarotation, kann also in einer $\alpha$ - und $\beta$-Form auftreten. Ist die Hydroxy-Gruppe am $\mathrm{C}_{1^{\prime}}$-Atom äquatorial ausgerichtet, so liegt die $\beta$-Form vor, entsprechend bei axialer Stellung die $\alpha$-Form. Am zweiten beteiligten Ring ist das anomere $\mathrm{C}$-Atom $\left(\mathrm{C}_{1}\right)$ durch die Sauerstoffbrücke blockiert, der Ring bricht nicht auf, es gibt also keine „Mutarotation“ und er bleibt stets in $\alpha$-Pyranose-Form. Die
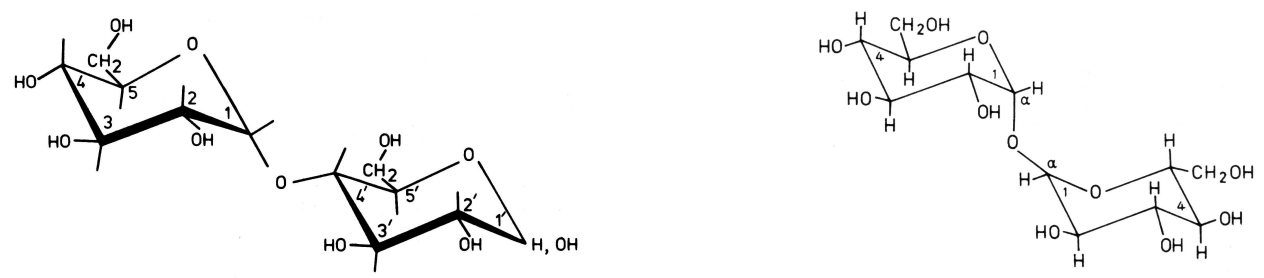

Abbildung 6.14: Maltose (links), Trehalose (rechts) [36][38].

$\alpha(1,4)$-glycosidische Bindung der Glucosemoleküle zur Maltose lässt sich beliebig um weitere Glucoseeinheiten erweitern und führt über die Maltotriose schließlich zur Amylose, einem Bestandteil der Stärke. Maltose ist ein wasserlösliches Zwischenprodukt beim Stärkeabbau und kommt z.B. in keimenden Getreide vor, das als Malz in der Bier oder Whiskyherstellung Verwendung findet, daher der Name Malzzucker.

\subsubsection{Trehalose}

Auch in der Trehalose sind zwei D-Glucose Moleküle miteinander verbunden. Allerdings sind bei ihr die anomeren $\mathrm{C}$-Atome beider Monosaccharide an der Brücke beteiligt und beide Glucopyranosen liegen in $\alpha$-Form vor $(\alpha(1,1)$-glycosidische Bindung). Trehalose ist mithin nichtreduzierend und zeigt keine Mutarotation. Die Bindungswinkel $\phi$ und $\psi$ sind austauschbar.

Trehalose kommt in der Natur als Hauptblutzucker der Insekten und Wirbellosen vor (bei Wirbeltieren: Glucose), und ist außerdem in Pilzen, Hefen und einigen Pflanzen zu finden. Neben der Transportfunktion als Blutzucker wird sie von Hefen und Insekten auch als Energiespeicher genutzt [36][38] und spielt eine wichtige Rolle beim Schutz von Zellen vor Schäden durch Gefrieren [55].

\subsubsection{Lactose}

Lactose wird aus je einem Molekül D-Galactose und D-Glucose gebildet. An der Bindung ist das anomere $\mathrm{C}$-Atom der $\beta$-Galactopyranose und die alkoholische Hydroxy-Gruppe des vierten $\mathrm{C}$-Atoms der Glucopyranose beteiligt, es liegt folgliche eine $\beta(1,4)$-glycosidische Bindung vor. Die Lactose ein reduzierendes Disaccharid und es tritt ein Mutarotationsgleichgewicht zwischen einer $\alpha$ - und $\beta$-Form auf. Lactose ist das wichtigste Kohlenhydrat in der Nahrung neugeborener Säuge- 

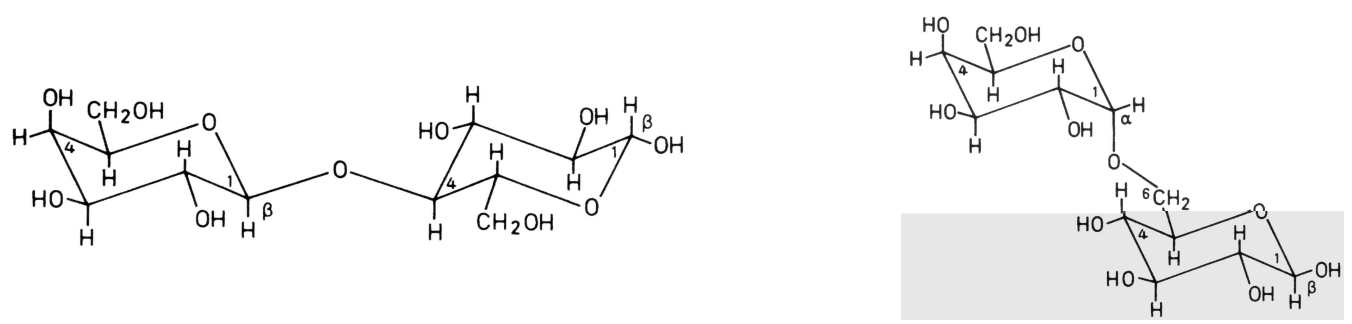

Abbildung 6.15: Lactose (links), Melibiose(rechts) [38].

tiere und Hauptzucker in der Milch, daher der Name Milchzucker. Dahingegen fehlt den meisten erwachsenen Menschen das lactoseabbauende Enzym Lactase, so dass Milchzucker nur zu ihrer Ernährung beitragen kann, wenn er z.B. durch Bakterien bei der Joghurt-Produktion abgebaut wird.

\subsubsection{Melibiose}

Melibiose nimmt unter vorgestellten Disacchariden eine Sonderrolle ein. Zum einen kommt sie in der Natur nicht frei, sondern z.B. als Teil des Trisaccharids Raffinose vor, aus dem sie auch gewonnen wird. Zum anderen erfolgt die Verknüpfung hier von einer $\alpha-\mathrm{D}$-Galactopyranose über das exocyclische $\mathrm{C}_{6}-$ Atom einer D-Glucopyranose, so dass eine $\alpha(1,6)$-glycosidische Bindung mit einer $\mathrm{O}-\mathrm{C}-$ Brücke zwischen den Monosaccharidringen entsteht. Damit bleibt die anomere Gruppe der Glucose frei, Melibiose ist ein reduzierendes Disaccharid, das Mutarotation zeigt. Außerdem gibt es hier drei nicht fixierte Einfachbindungen zwischen den Monosaccharideinheiten.

\subsubsection{Sucrose/Saccharose}

Die Sucrose, auch Saccharose genannt, ist das Disaccharid mit der größten Verbreitung bei Pflanzen. Sie kommt in vielen Früchten vor und wird industriell vor allem aus Zuckerrüben und Zuckerrohr („Rohrzucker“) gewonnen. Sucrose besteht aus der Verknüpfung von $\alpha$-D-Glucopyranose und $\beta$-D-Fructofuranose, die beide mit ihrer anomeren Gruppe an der Bindung beteiligt sind. Da die Fructose ein Ketozucker ist, bedeutet dies, dass das $\mathrm{C}_{2}$-Atom über die Sauerstoffbrücke mit dem $\mathrm{C}_{1}-$ Atom der Glucose verbunden ist. Das Fehlen der Lactol-Gruppen bewirkt wieder, dass ein nichtreduzierendes Disaccharid vorliegt, das keine Mutarotation zeigt. Weiterhin ist bei der Saccharose interessant, dass die Fructose in Form des fünfzähligen Furanoseringes beteiligt ist, während die Monosaccharide in allen anderen vorgestellten Disacchariden als Pyranoseringe vorliegen.

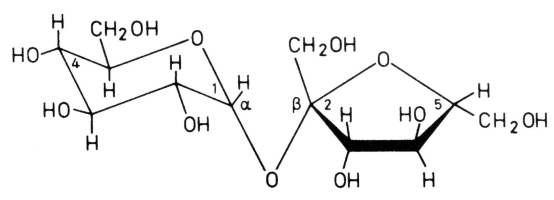

Abbildung 6.16: Sucrose / Saccharose [38]. 


\section{Auswertung}

Aufgrund physikalischer und empirischer Modelle der untersuchten Lösungen und ihrer Wechselwirkung mit Ultraschallwellen lassen sich jeweils charakteristische Frequenzabhängigkeiten der Absorption und z.T. der Schallgeschwindigkeits-Dispersion ableiten. Diese Spektralfunktionen bzw. eine Summe aus mehreren werden zusammen mit der ebenfalls zu bestimmenden Hintergrunddämpfung als Modellfunktion $\mathcal{S}\left(f, p_{1}, \ldots, p_{k}\right)$ mit den Parametern $p_{1}, \ldots, p_{k}$ an die Messdaten angepasst. Die Anpassung erfolgt nach dem Gauß-Verfahren der kleinsten Fehlerquadrate unter Verwendung des Marquardt-Algorithmus [45]. Bei den Messdaten - und damit auch bei $\mathcal{S}$ - wird die Darstellung als Dämpfung längs einer Wellenlänge $(\alpha \lambda)_{\text {ges ver- }}$ wendet, so dass die Hintergrunddämpfung in der Form $B \cdot f$ auftritt, die Differenz zur Gesamtdämpfung wird als Exzess-Dämpfung $(\alpha \lambda)_{\text {exc }}$ bezeichnet. Die Hintergrunddämpfung beinhaltet alle jenseits des Messbereiches hochfrequent relaxierenden Prozesse. Eine Anpassung an die $(\alpha \lambda)_{\text {ges }}$-Daten wird gewählt, da einige der Spektralfunktionen nur in dieser Form verfügbar sind. Um sicherzustellen, dass beim Anpassen der Modellfunktion an die Messdaten eine gleichmäßige Gewichtung über den Frequenzbereich erfolgt, werden die zur Frequenz $f_{i}$ gemessenen Absorptionen $(\alpha \lambda)_{\text {ges,i }}$ mit ihren Absolutfehlern gewichtet. Diese werden aus den $(\alpha \lambda)_{\text {ges }, \mathrm{i}}$ und den in den Tabellen 3.2 und 3.4 angegebenen relativen Fehlern berechnet. Ohne eine Gewichtung bzw. bei einer Gewichtung mit den relativen Fehlern würde eine Anpassung aufgrund der Hintergrunddämpfung in der $(\alpha \lambda)_{\text {ges }}$-Darstellung den hochfrequenten Teil des Spektrums betonen, eine Anpassung an die frequenznormierten $\left(\alpha / f^{2}\right)$-Daten hingegen den tieffrequenten.

Zur graphischen Veranschaulichung der gemessenen Frequenzverläufe der Ultraschallabsorption werden beide oben genannten Darstellungen der Daten verwendet.

Zunächst ist die doppeltlogarithmische Auftragung der Dämpfung $\alpha$ dividiert durch das Quadrat der Frequenz $f \mathrm{zu}$ nennen. In dieser Darstellung ist die quadratisch mit der Frequenz steigende Hintergrunddämpfung eine Parallele zur Frequenzachse, der Funktionswert fern von Relaxationsfrequenzen ist konstant und die Zusatzabsorption ist in Form fallender Stufen zu erkennen, deren Wendepunkte bei der jeweiligen Relaxationsfrequenz liegen. Somit sind Relaxationen bereits ohne Anpassung von Spektralfunktionen erkennbar und die $\left(\alpha / f^{2}\right)$-Werte konvergieren für große Frequenzen gegen den $B$-Wert der Hintergrunddämpfung: $\left(\alpha / f^{2}\right) \underset{f \rightarrow \infty}{\longrightarrow} B / c_{s}$.

Zum anderen wird die ebenfalls doppeltlogarithmische Auftragung der Zusatzabsorption längs einer Wellenlänge $(\alpha \lambda)_{\text {exc }}$ verwendet. Diese ist erst nach dem Anpassen geeigneter Spektralfunktionen und der Hintergrunddämpfung zugänglich, bietet aber den Vorteil, dass die Maxima der Exzess-Absorption und damit bei Verwendung von Debye-Spektralfunktionen die Relaxationsfrequenzen und Amplituden erkennbar sind, solange der Frequenzabstand der einzelnen Relaxationsterme groß genug ist. Da beim Ermitteln der Exzess-Absorption im oberen Messbereich mit der Gesamtdämpfung und der Hintergrunddämpfung zwei große Zahlen von- 
einander subtrahiert werden, zeigen die hochfrequenten Messpunkte auch bei guter Messgenauigkeit eine größere statistische Abweichung. Bei gleicher Achsenskalierung erfolgt der Anstieg der Debye-Funktion mit der Steigung eins, der Abfall mit minus eins.

Im Falle aller Saccharidspektren wurden zum Anpassen der Absorptionsverläufe ausschließlich Debye-Funktionen verwendet, da bisher in der Literatur kein Hinweis auf molekulare Prozesse mit einer kontinuierlichen Relaxationszeitverteilung, wie z.B. kritische oder vorkritische Konzentrationsfluktuationen, erscheint. Aus mathematischer Sicht lassen sich die Spektren auch unter Verwendung der empirischen Hill-Funktionen, mit einer reduzierten Zahl an freien Parametern anpassen, allerdings fehlt hierbei eine physikalische Begründung für ihre Anwendung.

\subsection{Disaccharide}

Die Auswertung und Analyse der Ultraschallabsorptionsspektren der wässrigen Disaccharid-Lösungen bereitet einige Schwierigkeiten. Um zu einer zufriedenstellenden Beschreibung der Messdaten durch eine Summe von Spektralfunktionen zu gelangen, sind durchgehend mindestens drei Debye-Terme erforderlich. Diese sind allerdings nicht, wie z.B. bei der wässrigen Fructoselösung (siehe Abbildung A.2), durch klare Maxima oder zumindest „Schultern“ im Absorptionsverlauf zu identifizieren. Daher ist eine systematische Variation der thermodynamischen Größen Konzentration und Temperatur erforderlich, um die Konsistenz der Anpassparameter zu überprüfen. Auch ein Vergleich der verschiedenen Disaccharide untereinander und mit den Spektren der jeweiligen an der Dimerisierung beteiligten Monosacchariden erweist sich als hilfreich, um die Charakteristika der Schallabsorption zu erfassen und eine Zuordnung der Spektralterme zu molekularen Prozessen zu ermöglichen.

\subsubsection{Maltose}

Die Maltose besteht aus zwei $\alpha(1,4)$-glycosidisch verknüpften Glucopyranosen. Wie die Auftragung der Exzess-Absorption längs einer Wellenlänge $(\alpha \lambda)_{\text {exc }}$ in Abbildung A.1 zeigt, lässt sich das Spektrum einer 1-molaren wässrigen Glucoselösung durch Anpassung zweier Debye-Terme und der Hintergrunddämpfung zufriedenstellend beschreiben. Da die Konzentration der "Glucoseringe“ im Falle der 0,5-molaren Maltose derjenigen der 1-molaren Glucose entspricht, wurde auch bei der Maltose eine Anpassung mit zwei Debye-Termen ausgeführt. Bei dieser zeigen die Messdaten jedoch eine merkliche systematische Abweichung von der Summenfunktion, wie die Abbildung 7.1 verdeutlicht. Eine Anpassung mit nur zwei Debye-Funktionen und der Hintergrunddämpfung reicht mithin für eine Beschreibung des Spektrums der Maltose nicht aus, so dass im Folgenden ein zusätzlicher dritter Debye-Term hinzugenommen wurde. Wie die Abbildung 7.2 zeigt, ist hiermit eine gute Wiedergabe des Absorptionsverlaufes möglich, die Messdaten streuen ohne nennenswerte Systematik im Rahmen der Fehler der jeweiligen Apparaturen um die Modellfunktion. 

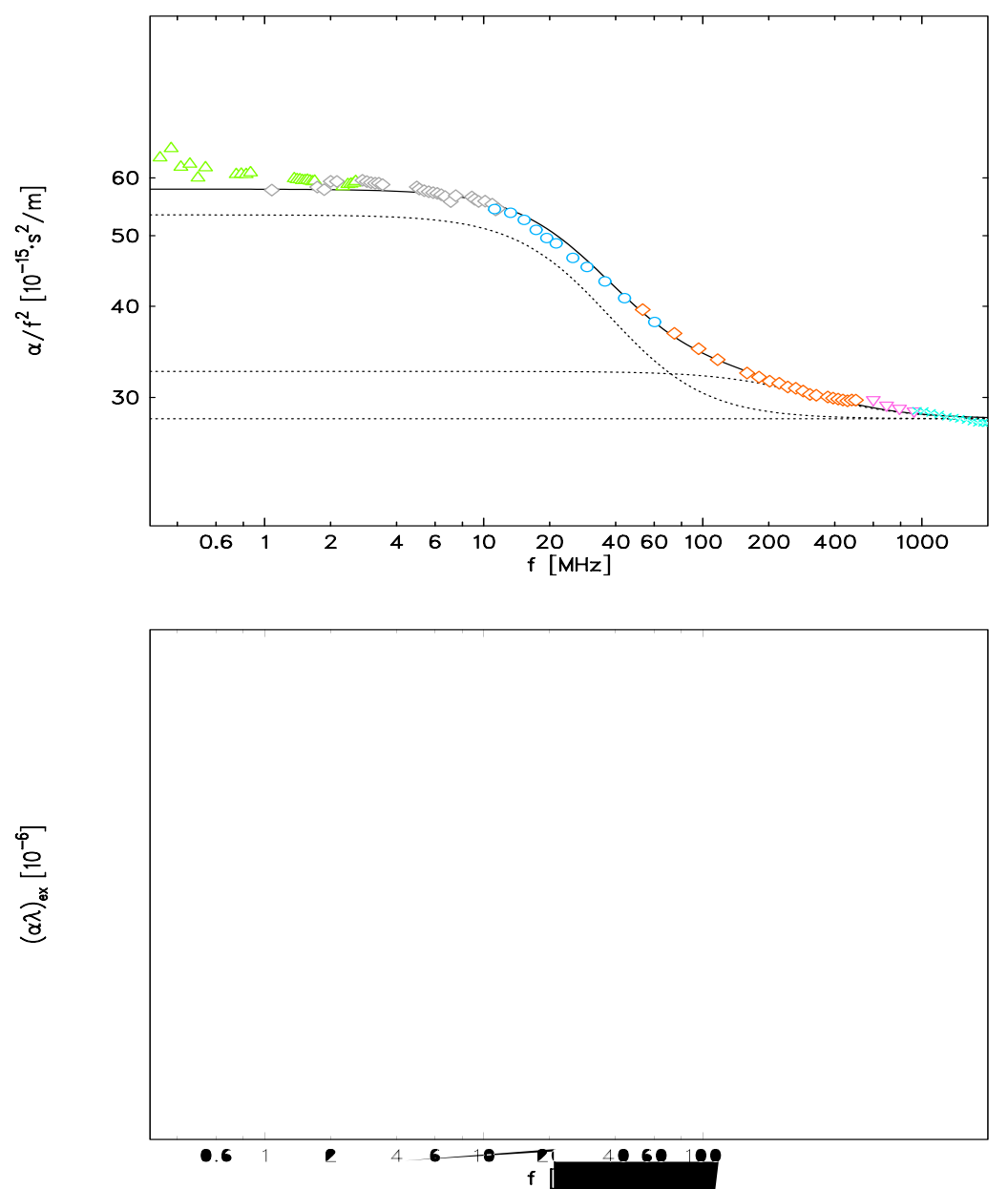

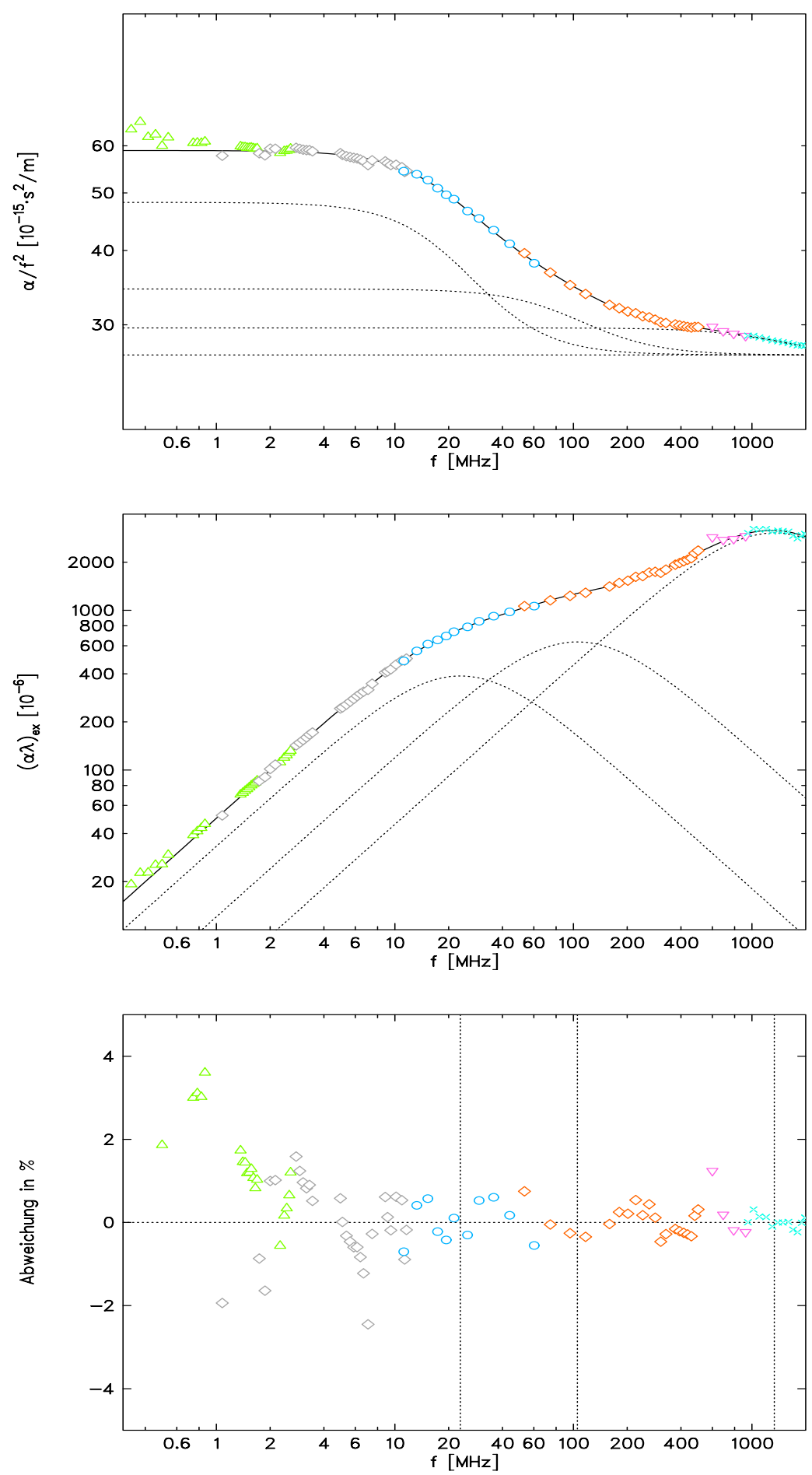

Abbildung 7.2: $\left(\alpha / f^{2}\right)$ - und $(\alpha \lambda)_{\text {exc }}$-Darstellung des Spektrums von $0,5-$ molarer wässriger Maltoselösung. Die durchgezogene Linie ist hier das Resultat der Anpassung einer Summe aus drei Debye-Spektraltermen (und der Hintergrunddämpfung bei der $\left(\alpha / f^{2}\right)$-Darstellung), die gestrichelten Kurven zeigen die einzelnen Summanden. Die untere Abbildung stellt die Abweichung der Messdaten von der Modellfunktion dar. Die Daten der einzelnen Messzellen werden durch die folgenden Symbole wiedergegeben (von links nach rechts): $\triangle: 1-\mathrm{MHz}$-Resonator, $\diamond: 4-\mathrm{MHz}-$ Resonator, $\circ$ : $1-\mathrm{MHz}-$ Pulszelle, $\diamond: 10-\mathrm{MHz}-$ Pulszelle, $\nabla$ : tieffrequente Hyperschallzelle, $\times:$ PZTZelle. 


\section{Konzentrationsabhängigkeit der Anpassparameter bei Maltose}

Um eine Zuordnung der angepassten Relaxationsterme zu verschiedenen Klassen von molekularen Prozessen, wie z.B. Konformationsänderungen, Assoziation, Fluktuation etc. zu ermöglichen und die Systematik der Anpassung zu überprüfen, wurde bei weiteren Messungen die Konzentration der Maltose in drei Schritten erhöht. Die höchste Konzentration von 1,8 mol/l wurde so gewählt, dass die aus den vorhergehenden Messungen abgeschätzte Schallgeschwindigkeit der Maltoselösung durch eine Harnstoff-Referenzlösung für die Resonatoren noch erreicht werden konnte. Die Konzentration von 10,44 mol/l der verwendeten Harnstoff-Lösung lag dabei für $25^{\circ} \mathrm{C}$ dicht an der Löslichkeitsgrenze. In Abbildung 7.3 ist die Zusatzabsorption längs einer Wellenlänge für die verschiedenen Konzentrationen dargestellt, die Parameter der Anpassung dreier Debye-Terme sind in Tabelle 7.1 aufgelistet. Da eine Zuordnung des Debye-Termes mit einer Relaxationszeit im Bereich von 1-2 ns zur Rotation der exocyclischen Hydroxymethyl-Gruppe möglich ist (vgl. Abschnitt 8.2), wurde in einer zweiten Anpassung die zugehörige Relaxationszeit auf 2 ns festgehalten, um die Signifikanz der übrigen Parameter zu erhöhen. Der Wert von 2 ns für $\tau_{2}$ ergibt sich als Mittelwert aus einer Zwei-Debye-Anpassung an konzentrationsabhängige Messungen von wässrigen Glucoselösungen [59][21].

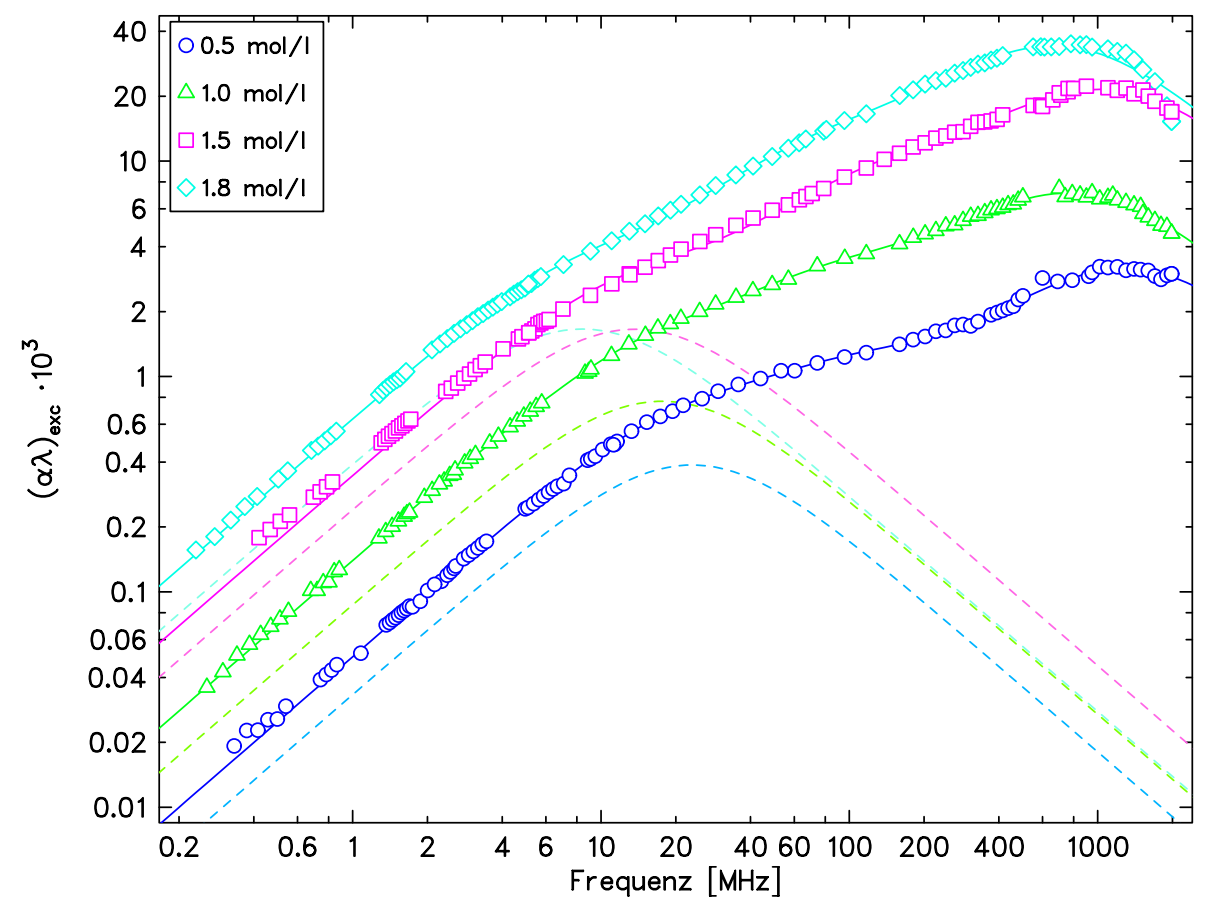

Abbildung 7.3: $(\alpha \lambda)_{\mathrm{exc}}-$ Darstellung der Spektren wässriger Maltoselösungen bei $25^{\circ} \mathrm{C}$. Dargestellt sind die Messdaten, der tieffrequente Debye-Term (--) und eine Summe aus drei Debye-Spektraltermen (-).

\section{Temperaturabhängigkeit der Anpassparameter der Maltose}

Zur Abschätzung thermodynamischer Parameter der molekularen Prozesse, die den Debye-Termen zugrundeliegenden, wurde außerdem die 0,5-molare Maltoselösung 


\begin{tabular}{|c|c|c|c|c|c|c|c|}
\hline $\begin{array}{c}\mathrm{c} \\
{[\mathrm{mol} / \mathrm{l}]}\end{array}$ & $\begin{array}{c}\tau_{1} \\
{[\mathrm{~ns}]}\end{array}$ & $\begin{array}{c}\mathrm{A}_{1} \\
{\left[10^{-3}\right]}\end{array}$ & $\begin{array}{c}\tau_{2} \\
{[\mathrm{~ns}]}\end{array}$ & $\begin{array}{c}\mathrm{A}_{2} \\
{\left[10^{-3}\right]}\end{array}$ & $\begin{array}{c}\tau_{3} \\
{[\mathrm{~ns}]}\end{array}$ & $\begin{array}{c}\mathrm{A}_{3} \\
{\left[10^{-3}\right]}\end{array}$ & $\begin{array}{c}\mathrm{B} \\
{[\mathrm{ps}]}\end{array}$ \\
\hline \multicolumn{1}{|l|}{ Anpassung mit freiem $\tau_{2}$} \\
\hline 0,5 & $6,8(2)$ & $0,78(4)$ & $1,51(9)$ & $1,27(4)$ & $0,12(2)$ & $6(2)$ & $41,4(5)$ \\
1,0 & $9,1(2)$ & $1,53(5)$ & $1,71(6)$ & $3,39(7)$ & $0,20(1)$ & $13(1)$ & $61,2(3)$ \\
1,5 & $11,6(4)$ & $3,31(1)$ & $1,13(6)$ & $9,7(5)$ & $0,15(2)$ & $40(6)$ & $101(2)$ \\
1,8 & $19,0(6)$ & $3,32(11)$ & $1,92(7)$ & $12,4(3)$ & $0,23(1)$ & $65(2)$ & $146(1)$ \\
\hline Anpassung mit festem $\tau_{2}=2 \mathrm{~ns}$ \\
\hline 0,5 & $7,7(3)$ & $0,61(2)$ & 2 & $1,22(4)$ & $0,19(2)$ & $3,7(4)$ & $42,3(2)$ \\
1,0 & $9,8(2)$ & $1,33(3)$ & 2 & $3,27(6)$ & $0,22(1)$ & $12,4(4)$ & $61,7(2)$ \\
1,5 & $14,1(6)$ & $2,59(9)$ & 2 & $6,1(2)$ & $0,28(2)$ & $26(1)$ & $109(1)$ \\
1,8 & $19,5(5)$ & $3,22(6)$ & 2 & $12,2(2)$ & $0,24(1)$ & $65(2)$ & $147(1)$ \\
\hline
\end{tabular}

Tabelle 7.1: Paramter der Anpassung von drei Debye-Termen an die Spektren der Maltoselösungen verschiedener Konzentration bei $25^{\circ} \mathrm{C}$.

bei vier Temperaturen zwischen $10^{\circ} \mathrm{C}$ und $35^{\circ} \mathrm{C}$ gemessen. Dieses entspricht im Wesentlichen dem maximalen Temperaturbereich, in dem die Temperaturkonstanz hinreichend gewährleistet ist und in dem die mechanische Funktion der Messzellen durch die unterschiedliche thermische Ausdehnung der Materialien beweglicher Teile und Dichtungen akzeptabel bleibt. In Abbildung 7.4 ist die Dämpfung längs einer Wellenlänge für die Temperaturserie dargestellt und Tabelle 7.2 enthält die Daten einer Anpassung der Spektren mit drei Debye-Termen. Da die Relaxationsfrequenz des hochfrequenten Debye-Termes dicht an der oberen Grenze des Messfrequenzbereiches liegt, sind dessen Parameter mit dem der Hintergrunddämpfung korreliert. Um auch hier einen glatten Parameterverlauf zu erreichen, wurde bei der höchsten Temperatur die Relaxationszeit $\tau_{3}$ festgehalten. Die Zeiten und Amplituden der beiden anderen Debye-Prozesse bleiben im Rahmen der Fehler hierdurch unbeeinflusst.

\begin{tabular}{|l|c|c|c|c|c|c|c|}
\hline $\begin{array}{c}\theta \\
{ }^{\circ} \mathrm{C}\end{array}$ & $\begin{array}{c}\tau_{1} \\
{[\mathrm{~ns}]}\end{array}$ & $\begin{array}{c}\mathrm{A}_{1} \\
{\left[10^{-3}\right]}\end{array}$ & $\begin{array}{c}\tau_{2} \\
{[\mathrm{~ns}]}\end{array}$ & $\begin{array}{c}\mathrm{A}_{2} \\
{\left[10^{-3}\right]}\end{array}$ & $\begin{array}{c}\tau_{3} \\
{[\mathrm{~ns}]}\end{array}$ & $\begin{array}{c}\mathrm{A}_{3} \\
{\left[10^{-3}\right]}\end{array}$ & $\begin{array}{c}\mathrm{B} \\
{[\mathrm{ps}]}\end{array}$ \\
\hline \multicolumn{1}{|c|}{ Anpassung freiem $\tau_{2}$} \\
\hline 10 & $16(1)$ & $0,44(5)$ & $4,0(2)$ & $1,63(5)$ & $0,39(2)$ & $4,4(2)$ & $70,6(2)$ \\
15 & $9,0(3)$ & $0,91(5)$ & $1,8(1)$ & $1,50(6)$ & $0,21(2)$ & $5,2(5)$ & $57,8(3)$ \\
25 & $6,8(2)$ & $0,78(4)$ & $1,5(1)$ & $1,27(4)$ & $0,12(2)$ & $6,1(2)$ & $41,4(5)$ \\
35 & $6,0(4)$ & $0,50(5)$ & $1,3(1)$ & $1,52(4)$ & $0,07(*)$ & $7,9(1)$ & $31,0(3)$ \\
\hline
\end{tabular}

Tabelle 7.2: Parameter der Anpassung von drei Debye-Termen an die Spektren der 0,5-molaren Maltoselösung bei verschiedenen Temperaturen. Der mit einem * gekennzeichnete Parameter wurde bei der Anpassung festgehalten.

\subsubsection{Trehalose}

Zur weiteren Analyse der gemessenen Spektren wurde die Art der Verknüpfung der Glucopyranosen zu einem Disaccharid variiert. Bei der Trehalose sind beide Gluco- 


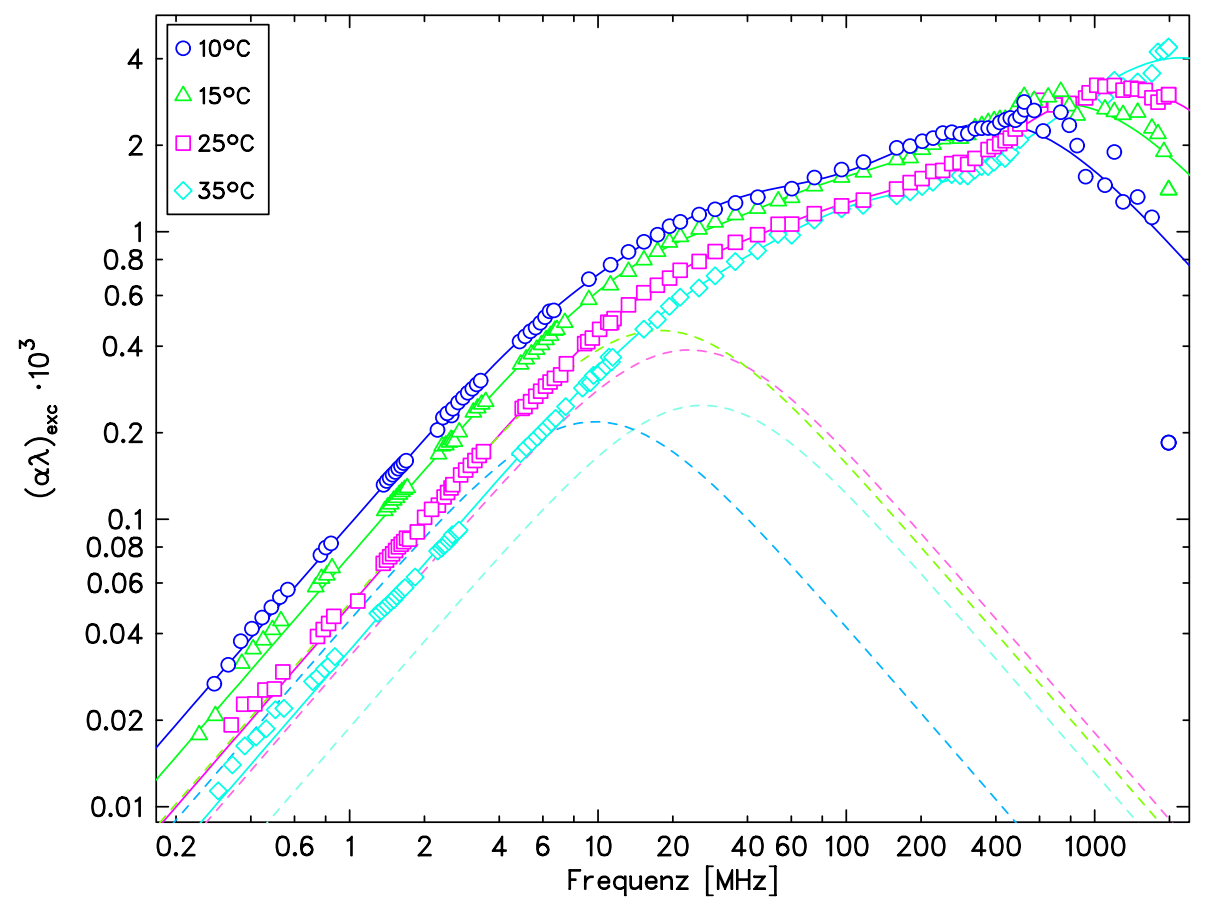

Abbildung 7.4: $(\alpha \lambda)_{\text {exc }}$-Darstellung der Spektren 0,5-molarer wässriger Maltoselösungen bei vier Temperaturen. Dargestellt sind die Messdaten, eine Summe aus drei Debye-Spektraltermen (-) und der tieffrequente Debye-Term (--).

seringe über ihre anomere Gruppe an der Bindung beteiligt, es liegt eine $\alpha(1,1)-$ glycosidische Bindung vor, $\phi$ und $\psi$ sind austauschbar, die Trehalose ist also „symmetrisch". Für die Trehalose wurde bei einer Konzentration von 1,0 mol/l ebenfalls bei vier Temperaturen von $10^{\circ} \mathrm{C}$ bis $35^{\circ} \mathrm{C}$ das Ultraschallabsorptionsspektrum gemessen. Wie im Falle der Maltose ist auch bei der Trehalose eine Anpassung mit nur zwei Debye-Relaxationstermen nicht ausreichend, während nach Einfügen eines zusätzlichen Termes die Qualität der Beschreibung zufriedenstellend ist. Die Parameter der drei Debye-Terme sind in Tabelle 7.3 aufgelistet und Abbildung 7.5 zeigt den Temperaturverlauf der Zusatzabsorptionsspektren.

\begin{tabular}{|c|c|c|c|c|c|c|c|}
\hline $\begin{array}{c}\theta \\
{ }^{\circ} \mathrm{C}\end{array}$ & $\begin{array}{c}\tau_{1} \\
{[\mathrm{~ns}]}\end{array}$ & $\begin{array}{c}\mathrm{A}_{1} \\
{\left[10^{-3}\right]}\end{array}$ & $\begin{array}{c}\tau_{2} \\
{[\mathrm{~ns}]}\end{array}$ & $\begin{array}{c}\mathrm{A}_{2} \\
{\left[10^{-3}\right]}\end{array}$ & $\begin{array}{c}\tau_{3} \\
{[\mathrm{~ns}]}\end{array}$ & $\begin{array}{c}\mathrm{A}_{3} \\
{\left[10^{-3}\right]}\end{array}$ & $\begin{array}{c}\mathrm{B} \\
{[\mathrm{ps}]}\end{array}$ \\
\hline 10 & $8,5(4)$ & $1,5(1)$ & $2,1(1)$ & $3,9(1)$ & $0,24(2)$ & $10,7(7)$ & $105,3(4)$ \\
15 & $6,5(3)$ & $1,6(1)$ & $1,4(1)$ & $3,7(1)$ & $0,14(2)$ & $13,1(2)$ & $86,0(9)$ \\
25 & $5,4(3)$ & $0,9(1)$ & $1,3(1)$ & $3,0(1)$ & $0,12(*)$ & $11,1(5)$ & $60,1(2)$ \\
35 & $4,2(4)$ & $0,6(1)$ & $0,9(1)$ & $3,0(1)$ & $0,10(4)$ & $6(4)$ & $46,0(1)$ \\
\hline
\end{tabular}

Tabelle 7.3: Parameter der Anpassung von drei Debye-Termen an die Spektren der 1,0-molaren Trehaloselösung bei vier Temperaturen. Der mit einem $*$ gekennzeichnete Parameter wurde bei der Anpassung festgehalten. 


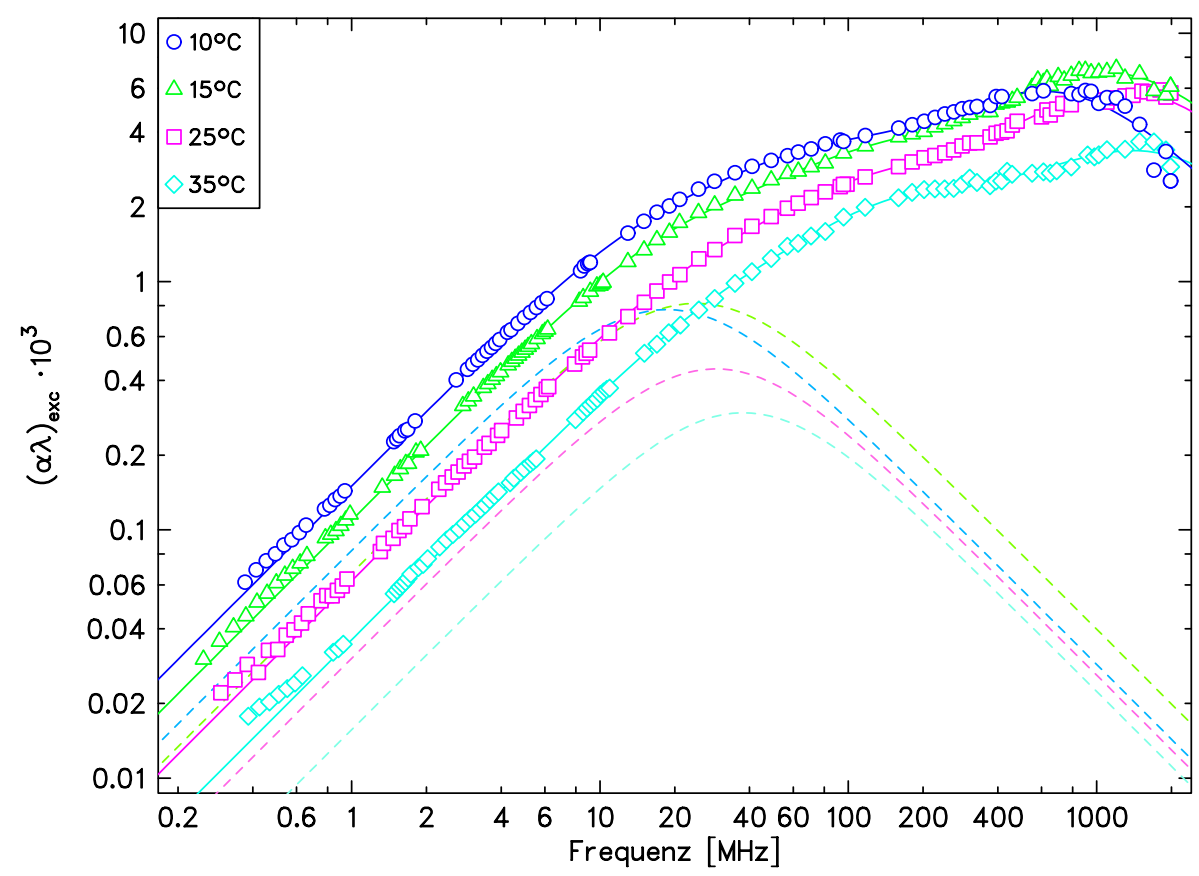

Abbildung 7.5: $(\alpha \lambda)_{\text {exc }}$-Darstellung der Spektren wässriger 1-molarer Trehaloselösungen bei vier Temperaturen. Dargestellt sind die Messdaten, der tieffrequente Debye-Term (--) und eine Summe aus drei Debye-Spektraltermen (-).

\subsubsection{Sucrose, Lactose und Melibiose}

Bei Saccharidketten stellt sich die Frage, ob die am Aufbau beteiligten Monosaccharideinheiten selbst einen wesentlichen Einfluss auf die Eigenschaften des Moleküls haben oder diese durch die Bindung zwischen den Ringen geprägt werden. Um dieser Fragestellung nachzugehen, wurden wässrige Lösungen von Disacchariden untersucht, die aus einer Glucopyranose und einem anderen Saccharid-Ring bestehen. Im Falle der Lactose handelt es sich beim zweiten Konstituenten um Galactopyranose die über ihre anomere Gruppe und eine Sauerstoffbrücke an das vierte Kohlenstoffatom der Glucose gebunden ist. Bei der Sucrose ist der zweite Ring eine Fructofuranose. Schließlich stellt die Melibiose einen Vertreter der Verküpfung über eine Kohlenstoff-Sauerstoffbrücke dar. Das zweite Monosaccharid ist eine Galactose. Hier wäre zusätzlich die Gentiobiose interessant gewesen, bei der eine solche Brücke zwischen zwei Glucopyranosen existiert, die mithin einen direkten Vergleich zur Maltose und Trehalose ermöglicht hätte. Leider ist dieser Zucker in den benötigten Mengen unverhältnismäßig teuer.

\section{Sucrose}

Die Sucrose ist das einzige gemessene Disaccharid, das einen Furanosering enthält. Trotz dieser Variation des zweiten Monosaccharids lässt sich das Spektrum einer 1,0-molaren wässrigen Sucroselösung ebenfalls mit drei Debye-Spektralfunktionen und der Hintergrunddämpfung beschreiben. Insbesondere wird kein tieffrequenter Debye-Term am unteren Ende des Messbereiches benötigt, wie dies bei der reinen Fructose der Fall ist. Die Relaxationsfrequenz des hochfrequenten Debye-Termes 


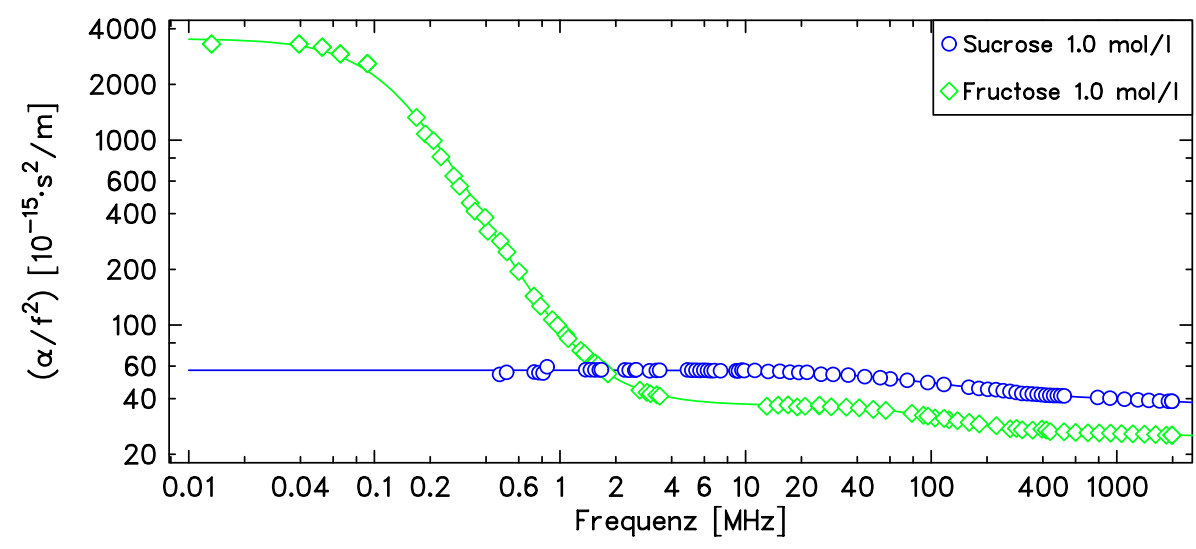

Abbildung 7.6: $\left(\alpha / f^{2}\right)$-Darstellung der Spektren von 1-molarer wässriger Sucrose$(\circ)$ und Fructoselösung $(\diamond)$ bei $25^{\circ} \mathrm{C}$. Die durchgezogenen Linien zeigen jeweils das Ergebnis einer Anpassung aus drei Debye-Termen und der Hintergrunddämpfung.

liegt mit ca. $2 \mathrm{GHz}$ genau an der Grenze des Messfrequenzbereiches. Daher sind die Parameter dieses Debye-Termes stark mit dem $B$-Wert korreliert, was sich in den wesentlich größeren Anpassfehlern - im Fall der Amplitude $A_{3}$ nahezu 100\% - widerspiegelt. Die Parameter aller Terme sind in Tabelle 7.4 enthalten und Abbildung 7.7 zeigt die Exzess-Absorption längs einer Wellenlänge.

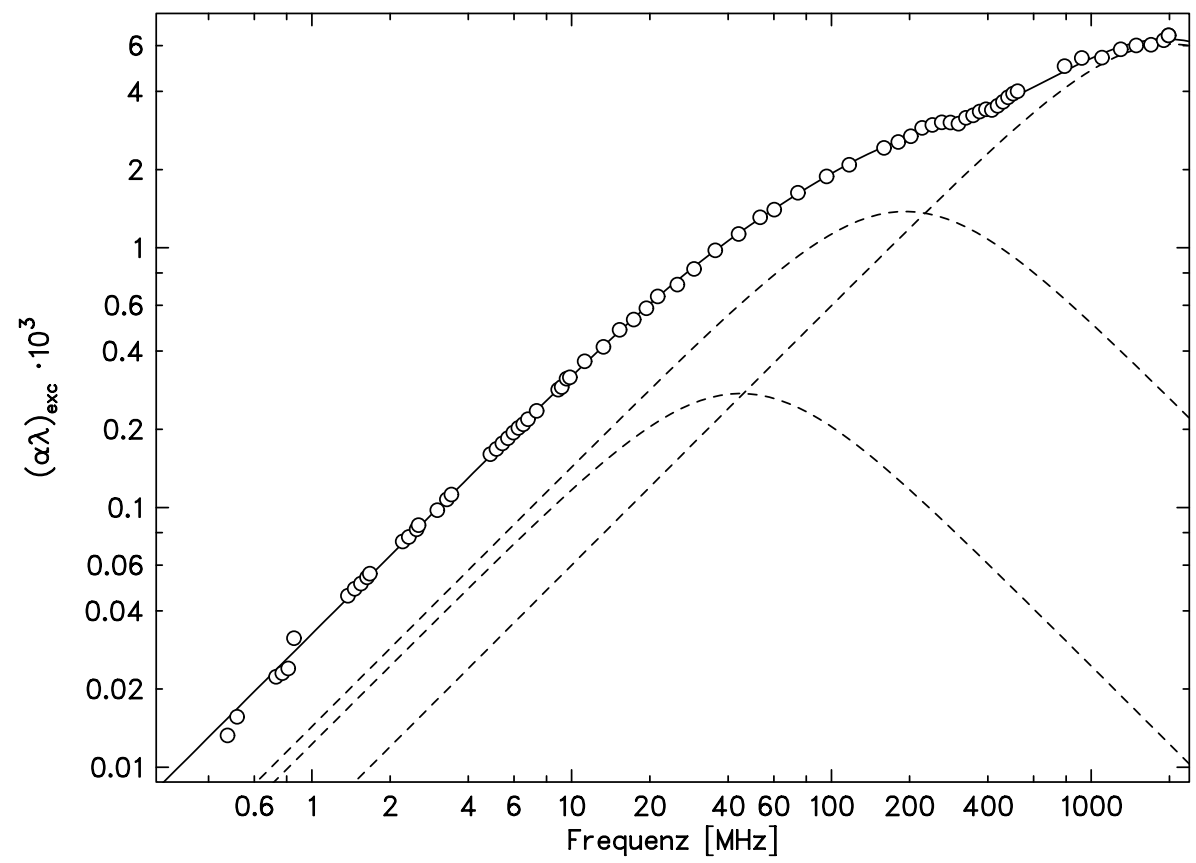

Abbildung 7.7: $(\alpha \lambda)_{\text {exc }}$-Darstellung des Spektrum von 1-molarer wässriger Sucroselösung bei $25^{\circ} \mathrm{C}$. Die durchgezogene Linie ist die angepasste Summe aus drei DebyeTermen, die gestrichelten Linien geben die einzelnen Summanden wieder. 


\begin{tabular}{|c|c|c|c|c|c|c|c|}
\hline $\begin{array}{c}\theta \\
{ }^{\circ} \mathrm{C}\end{array}$ & $\begin{array}{c}\tau_{1} \\
{[\mathrm{~ns}]}\end{array}$ & $\begin{array}{c}\mathrm{A}_{1} \\
{\left[10^{-3}\right]}\end{array}$ & $\begin{array}{c}\tau_{2} \\
{[\mathrm{~ns}]}\end{array}$ & $\begin{array}{c}\mathrm{A}_{2} \\
{\left[10^{-3}\right]}\end{array}$ & $\begin{array}{c}\tau_{3} \\
{[\mathrm{~ns}]}\end{array}$ & $\begin{array}{c}\mathrm{A}_{3} \\
{\left[10^{-3}\right]}\end{array}$ & $\begin{array}{c}\mathrm{B} \\
{[\mathrm{ps}]}\end{array}$ \\
\hline 25 & $3,6(3)$ & $0,55(8)$ & $0,83(5)$ & $2,8(1)$ & $0,08(3)$ & $12(9)$ & $59(2)$ \\
\hline
\end{tabular}

Tabelle 7.4: Parameter der Anpassung von drei Debye-Termen an das Spektrum der 1,0-molaren wässrigen Sucroselösung.

\section{Lactose}

Die Spektren der 0,5-molaren wässrigen Lösungen von Lactose bei $18^{\circ} \mathrm{C}, 25^{\circ} \mathrm{C}$ und $35^{\circ} \mathrm{C}$ zeigen gegenüber den bisher vorgestellten Disaccharidspektren jeweils einen weiteren, vierten Debye-Prozess am tieffrequenten Ende des Messbereiches, wie in Abbildung 7.8 in der $\left(\alpha / f^{2}\right)$-Darstellung gut zu erkennen ist. Die Parameter der Anpassung können Tabelle 7.5 entnommen werden.

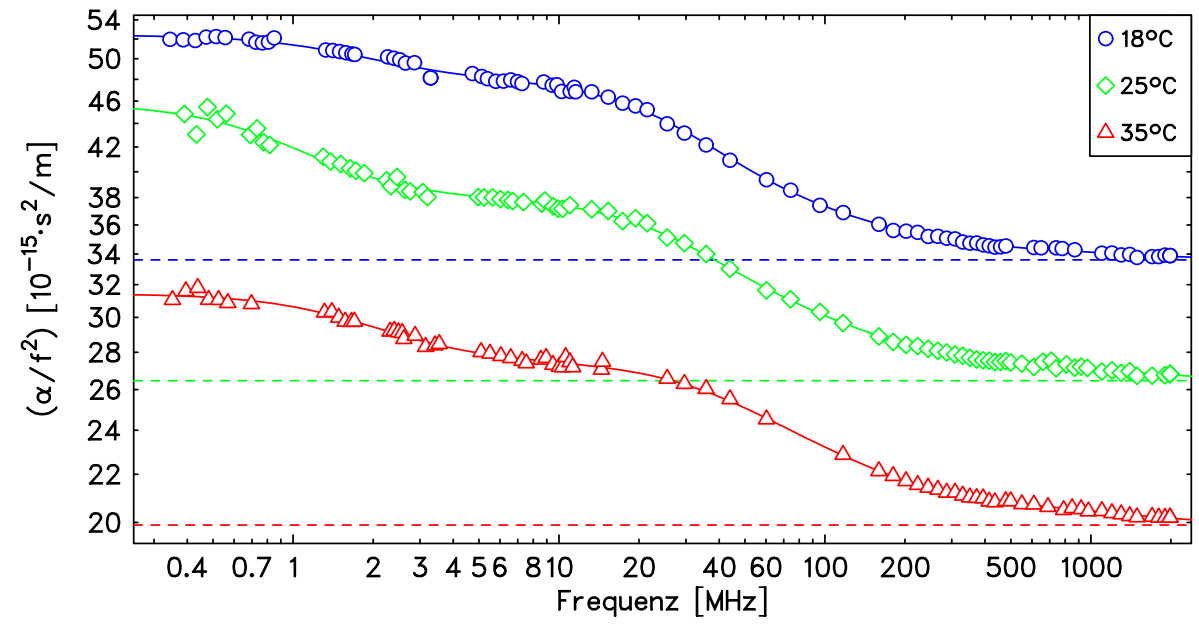

Abbildung 7.8: $\left(\alpha / f^{2}\right)$-Darstellung der Spektren von 0,5-molarer wässriger Lactoselösung bei $18^{\circ} \mathrm{C}(\circ), 25^{\circ} \mathrm{C}(\diamond)$ und $35^{\circ} \mathrm{C}(\triangle)$. Die durchgezogenen Linien geben die Summe aus jeweils vier Debye-Termen und der Hintergrunddämpfung, die gestrichelteten Linien die Hintergrunddämpfung alleine wieder.

\begin{tabular}{|c|c|c|c|c|c|c|c|c|c|}
\hline $\begin{array}{c}\theta \\
{ }^{\circ} \mathrm{C}\end{array}$ & $\begin{array}{c}\tau_{1} \\
{[\mathrm{~ns}]}\end{array}$ & $\begin{array}{c}\mathrm{A}_{1} \\
{\left[10^{-3}\right]}\end{array}$ & $\begin{array}{c}\tau_{2} \\
{[\mathrm{~ns}]}\end{array}$ & $\begin{array}{c}\mathrm{A}_{2} \\
{\left[10^{-3}\right]}\end{array}$ & $\begin{array}{c}\tau_{3} \\
{[\mathrm{~ns}]}\end{array}$ & $\begin{array}{c}\mathrm{A}_{3} \\
{\left[10^{-3}\right]}\end{array}$ & $\begin{array}{c}\tau_{0} \\
{[\mathrm{~ns}]}\end{array}$ & $\begin{array}{c}\mathrm{A}_{0} \\
{\left[10^{-3}\right]}\end{array}$ & $\begin{array}{c}\mathrm{B} \\
{[\mathrm{ps}]}\end{array}$ \\
\hline 18 & $4,5(2)$ & $0,61(4)$ & $1,0(1)$ & $0,63(5)$ & $0,11(6)$ & $1,5(12)$ & $85(12)$ & $0,013(2)$ & $51,7(4)$ \\
25 & $4,4(2)$ & $0,44(4)$ & $1,1(1)$ & $0,65(4)$ & $0,10(4)$ & $2,0(14)$ & $155(19)$ & $0,013(1)$ & $41,2(4)$ \\
35 & $3,1(4)$ & $0,34(1)$ & $1,1(2)$ & $0,56(7)$ & $0,11(3)$ & $1,7(7)$ & $80(7)$ & $0,0125(1)$ & $31,3(3)$ \\
\hline
\end{tabular}

Tabelle 7.5: Parameter der Anpassung von vier Debye-Termen an die Spektren der 0,5-molaren wässrigen Lactoselösung bei verschieden Temperaturen.

\section{Melibiose}

Bei der Melibiose reichen hingegen wieder drei Debye-Terme aus, um den Absorptionsverlauf zufriedenstellend wiederzugeben. Allerdings zeigt sich wie bei der Lacto- 
se ein zusätzlicher Debye-Prozess am tieffrequenten Ende des Messbereiches, dafür entfällt der Relaxationsterm mit einer Zeit von ca. 1-2 ns (vgl. Abbildung 7.10). Abbildung 7.9 zeigt den Absorptionsverlauf für vier Temperaturen in der $(\alpha \lambda)_{\mathrm{exc}^{-}}$ Darstellung und jeweils die drei angepassten Debye-Terme, deren Parameter können Tabelle 7.6 entnommen werden.

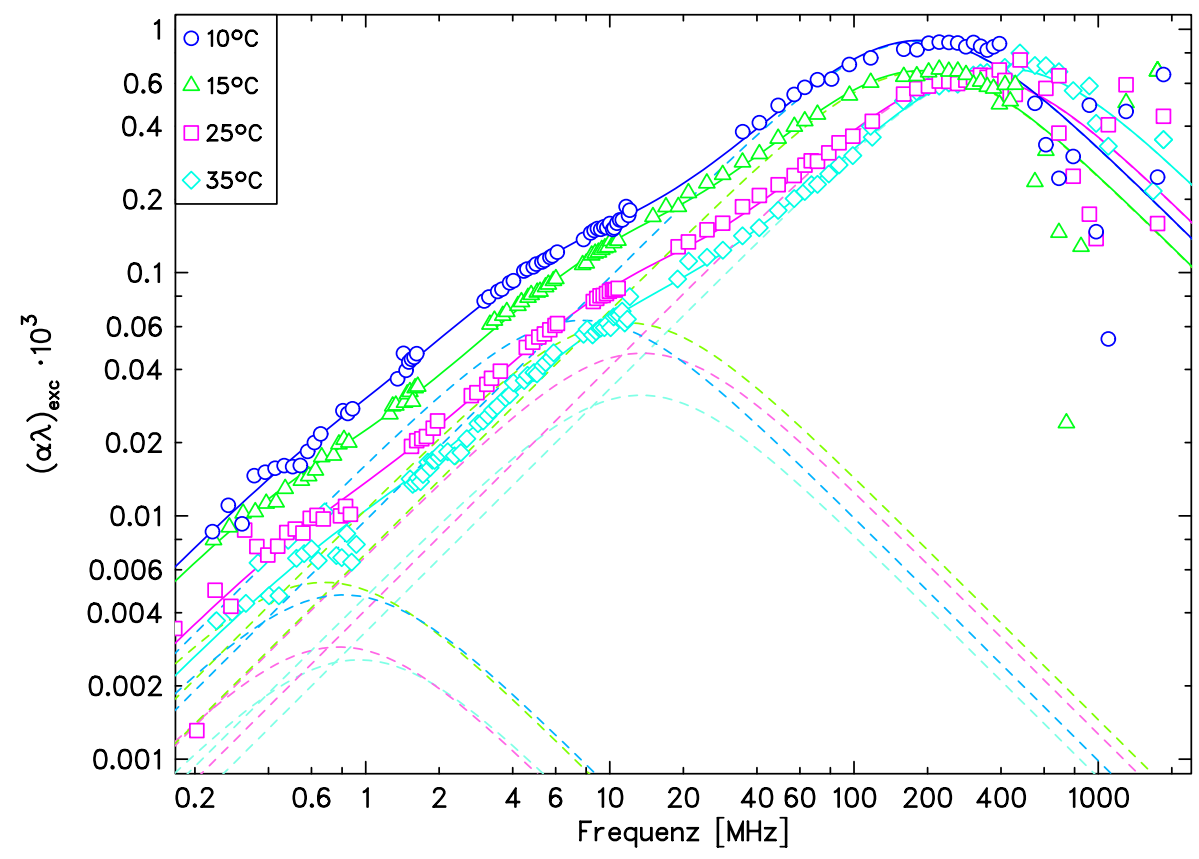

Abbildung 7.9: $(\alpha \lambda)_{\text {exc }}$-Darstellung der Spektren von 0,5-molarer wässriger Melibioselösung bei $10^{\circ} \mathrm{C}(\circ), 15^{\circ} \mathrm{C}(\diamond), 25^{\circ} \mathrm{C}(\diamond)$ und $35^{\circ} \mathrm{C}(\triangle)$. Die durchgezogenen Linien geben die Summe aus jeweils drei Debye-Termen, die gestrichelteten Linien die einzelnen Debye-Terme wieder.

\begin{tabular}{|c|c|c|c|c|c|c|c|}
\hline $\begin{array}{c}\theta \\
{ }^{\circ} \mathrm{C}\end{array}$ & $\begin{array}{c}\tau_{0} \\
{[\mathrm{~ns}]}\end{array}$ & $\begin{array}{c}\mathrm{A}_{0} \\
{\left[10^{-3}\right]}\end{array}$ & $\begin{array}{c}\tau_{1} \\
{[\mathrm{~ns}]}\end{array}$ & $\begin{array}{c}\mathrm{A}_{1} \\
{\left[10^{-3}\right]}\end{array}$ & $\begin{array}{c}\tau_{3} \\
{[\mathrm{~ns}]}\end{array}$ & $\begin{array}{c}\mathrm{A}_{3} \\
{\left[10^{-3}\right]}\end{array}$ & $\begin{array}{c}\mathrm{B} \\
{[\mathrm{ps}]}\end{array}$ \\
\hline 10 & $196(69)$ & $0,009(3)$ & $21(2)$ & $0,13(1)$ & $0,85(3)$ & $1,8(1)$ & $71,1(1)$ \\
15 & $235(3)$ & $0,011(1)$ & $14(1)$ & $0,12(1)$ & $0,83(2)$ & $1,3(1)$ & $59,3(1)$ \\
25 & $205(67)$ & $0,006(2)$ & $12(2)$ & $0,09(1)$ & $0,51(3)$ & $1,3(1)$ & $42,8(1)$ \\
35 & $170(*)$ & $0,005(1)$ & $12(1)$ & $0,06(1)$ & $0,39(2)$ & $1,4(1)$ & $32,2(1)$ \\
\hline
\end{tabular}

Tabelle 7.6: Parameter der Anpassung von drei Debye-Termen an die Spektren der 0,5-molaren wässrigen Melibioselösung bei verschieden Temperaturen. Der mit einem * gekennzeichnete Parameter wurde bei der Anpassrechnung festgehalten. 

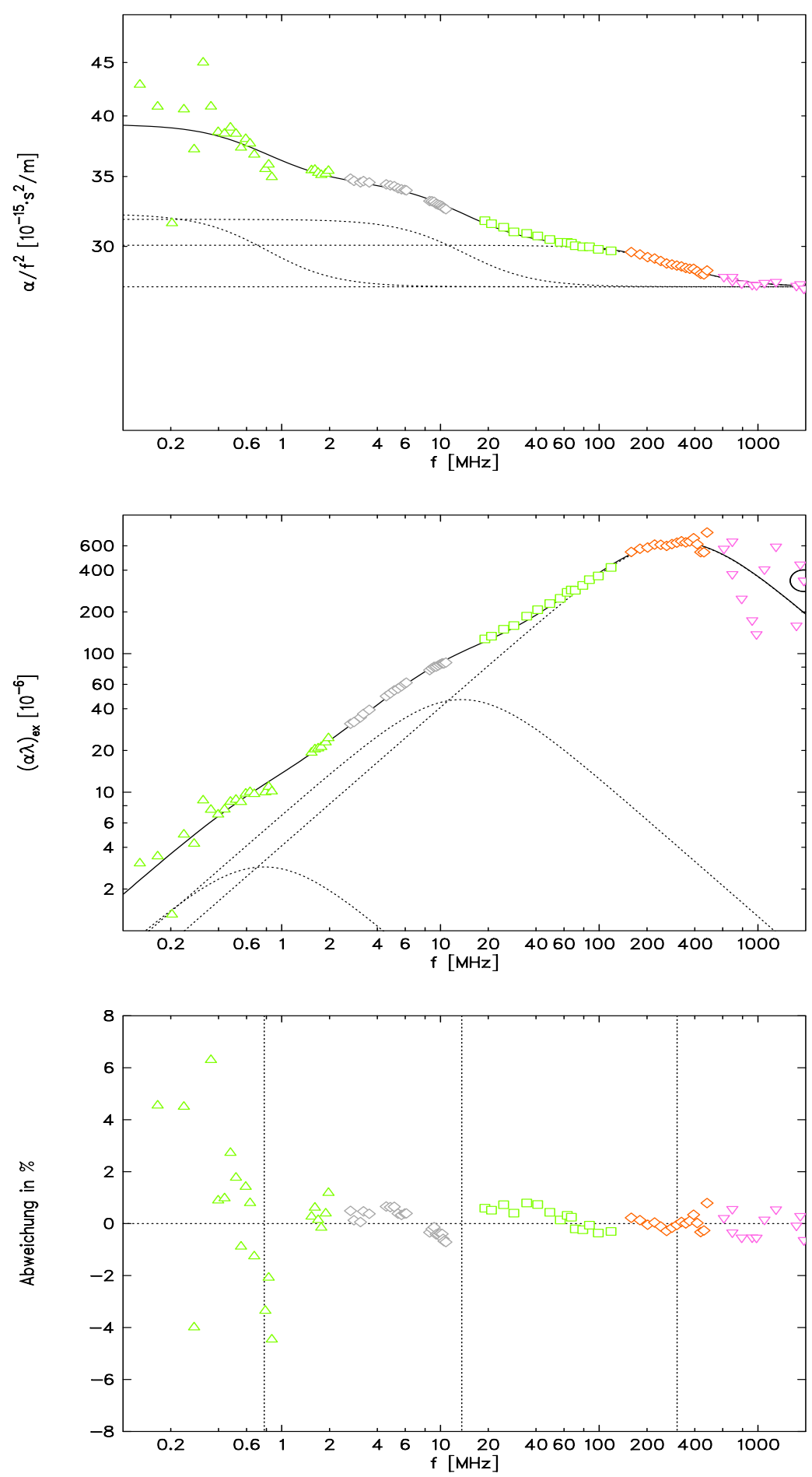

Abbildung 7.10: $\left(\alpha / f^{2}\right)-$ und $(\alpha \lambda)_{\text {exc }}-$ Darstellung des Spektrums von $0,5-$ molarer wässriger Melibioselösung. Die durchgezogene Linie ist die Summe einer Anpassung von drei Debye-Spektraltermen (und bei der $\left(\alpha / f^{2}\right)$-Darstellung der Hintergrunddämpfung). Die einzelnen Summanden sind durch gestrichelte Linien dargestellt. In der unteren Abbildung ist die Abweichung der Messdaten von der angepassten Modellfunktion aufgetragen. Die Daten der einzelnen Messzellen werden durch die folgenden Symbole wiedergegeben (von links nach rechts): $\triangle: 1-\mathrm{MHz}-$ Resonator, $\diamond: 4-\mathrm{MHz}-$ Resonator, $\circ: 1-\mathrm{MHz}-\mathrm{Pulszelle}, \diamond: 10-\mathrm{MHz}-\mathrm{Pulszelle}, \nabla:$ tieffrequente Hyperschallzelle, $\times$ : PZT-Zelle. Die eingekreisten Symbole wurden aus dem Negativen "gespiegelt". 


\subsection{Kohlenhydrate mit Calcium}

\subsubsection{Maltose}

Zur weiteren Untersuchung der Wechselwirkung von Kohlenhydraten mit CalciumIonen wurden die Ultraschallspektren einer 0,5-molaren Maltoselösung mit Zusatz von $0,25 \mathrm{~mol} / 1,0,5 \mathrm{~mol} / \mathrm{l}$ und 1,0 mol/l Calciumchlorid gemessen. Die folgende Abbildung 7.11 zeigt die Zusatzabsorption längs einer Wellenlänge für alle drei Konzentrationen. Auch für die Anpassung der Maltoselösungen mit Calciumzusatz ist - wie bei der reinen Maltoselösung - die Verwendung von drei Debye-Termen erforderlich, darüberhinaus werden keine Spektralterme zur Beschreibung des Absorptionsverlaufes benötigt. Dabei bleiben die Relaxationzeiten der einzelnen Debye-Terme im Rahmen der Fehler konstant, während die Amplituden gegenüber der reinen Maltose signifikant erhöht sind. In Tabelle 7.7 sind die Parameter der Ausgleichsrechnung mit drei Debye-Termen aufgeführt.

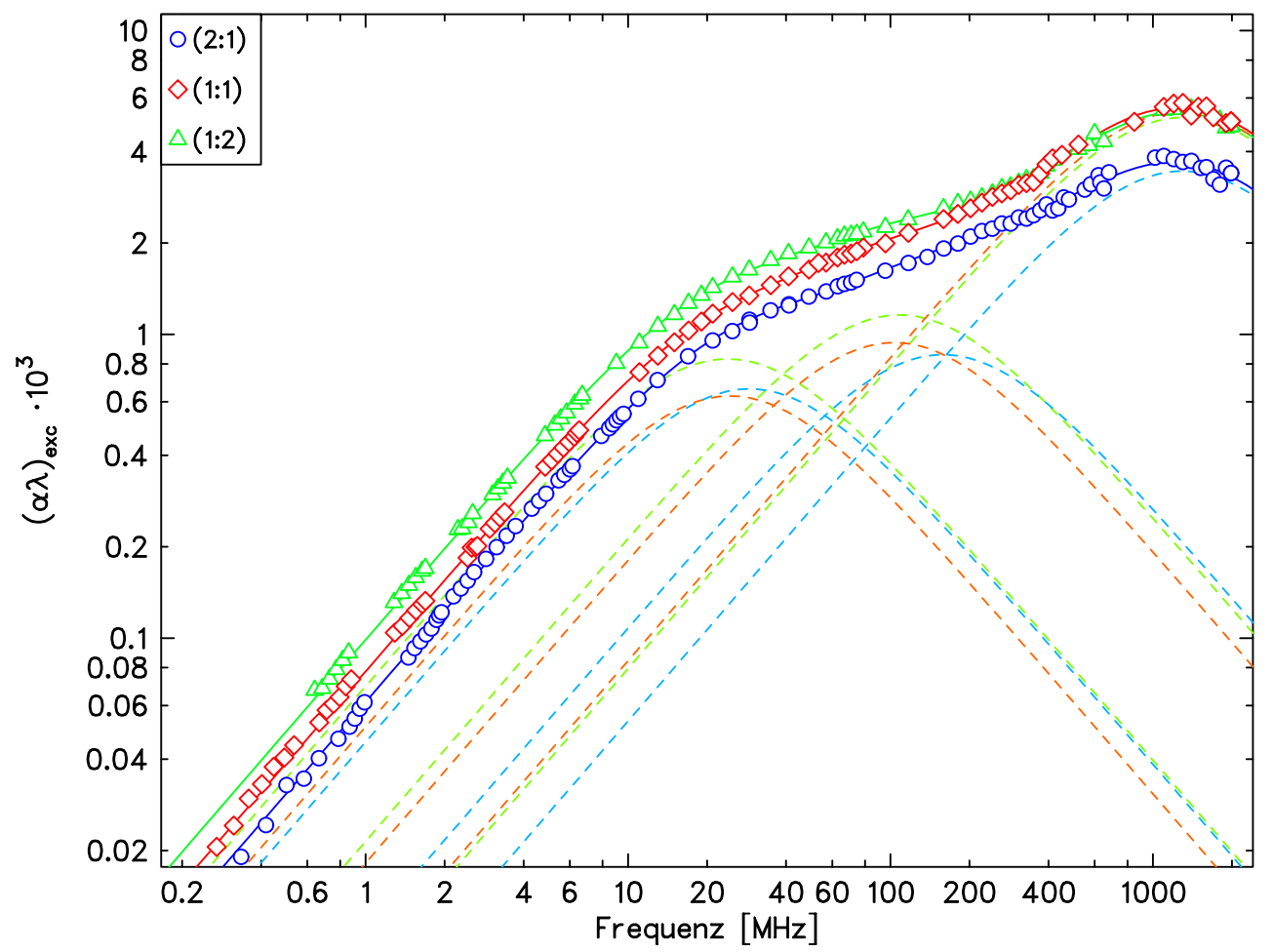

Abbildung 7.11: Exzess-Absorption von 0,5-molarer wässriger Maltoselösung mit Zusatz von $\mathrm{CaCl}_{2}$ in den Verhältnissen $2: 1,1: 1$ und $1: 2$ bei $25^{\circ} \mathrm{C}$.

\subsubsection{Fructose}

Im Gegensatz zur Maltose lassen sich die Spektren der Fructose bei einem Zusatz von Calciumchlorid verschiedener Konzentration nicht mehr durch die drei Debye-Terme der reinen wässrigen Fructoselösung beschreiben. Das Zusatzabsorptionsspektrum zeigt eine weitere, sehr ausgeprägte Relaxation bei einer Zeit von ca. 6 ns, so dass zur 


\begin{tabular}{|c|c|c|c|c|c|c|c|}
\hline $\begin{array}{c}\mathrm{c}_{\mathrm{Ca}^{2+}} \\
{[\mathrm{mol} / \mathrm{l}]}\end{array}$ & $\begin{array}{c}\tau_{1} \\
{[\mathrm{~ns}]}\end{array}$ & $\begin{array}{c}\mathrm{A}_{1} \\
{\left[10^{-3}\right]}\end{array}$ & $\begin{array}{c}\tau_{2} \\
{[\mathrm{~ns}]}\end{array}$ & $\begin{array}{c}\mathrm{A}_{2} \\
{\left[10^{-3}\right]}\end{array}$ & $\begin{array}{c}\tau_{3} \\
{[\mathrm{~ns}]}\end{array}$ & $\begin{array}{c}\mathrm{A}_{3} \\
{\left[10^{-3}\right]}\end{array}$ & $\begin{array}{c}\mathrm{B} \\
{[\mathrm{ps}]}\end{array}$ \\
\hline 0,25 & $5,48(10)$ & $1,32(4)$ & $1,00(5)$ & $1,72(7)$ & $0,123(17)$ & $6,9(13)$ & $43,1(5)$ \\
0,5 & $6,49(19)$ & $1,25(6)$ & $1,54(7)$ & $1,88(5)$ & $0,124(13)$ & $10,8(17)$ & $43,6(6)$ \\
1,0 & $6,66(13)$ & $1,66(5)$ & $1,47(6)$ & $2,32(5)$ & $0,123(14)$ & $10,3(17)$ & $49,6(6)$ \\
\hline
\end{tabular}

Tabelle 7.7: Parameter der Anpassung dreier Debye-Terme an die Absorptionsspektren von 0,5-molarer wässriger Maltoselösung mit Calciumchloridzusatz. Zum Vergleich sind die Parameter der reinen Maltose angegeben.

Anpassung der Spektren der wässrigen Fructoselösungen mit Calciumzusatz insgesamt vier Debye-Terme verwendet wurden. Da der Beitrag des „Calcium-Prozesses“ zur Zusatzabsorption bei tiefen Frequenzen sehr gering ist, wurde auf eine Messung mit dem Kugelresonator verzichtet und die Relaxationszeit der Sessel-Sessel Umwandlung der $\alpha$-D-Fructopyranose bei der Anpassung der Fructose/CalciumSpektren auf diejenige der reinen Fructoselösung festgehalten. Die Relaxationszeit der Rotation der exocyclischen Gruppe wurde ebenfalls auf den Wert der reinen Fructoselösung festgehalten, da ansonsten die Signifikanz der Parameter der drei hochfrequenten Debye-Terme erheblich reduziert wird. Die Debye-Terme bei ca. $6 \mathrm{~ns}, 2 \mathrm{~ns}, 0,1 \mathrm{~ns}$ und der B-Wert sind bei einer Anpassung mit freien Parametern stark korreliert. Da sowohl bei der Maltose als auch bei der Glucose bei einem Zusatz von Calcium die Relaxationszeit der Rotation der exocylischen Gruppe nicht signifikant beeinflusst wird (siehe Abschnitt 8.5.1), scheint dieses Vorgehen gerechtfertigt. Die folgende Abbildung zeigt die Exzess-Absorptions-Spektren der wässrigen 0,5molaren Fructoselösung mit Zusatz von $\mathrm{CaCl}_{2}$ mit den Konzentrationen 0,25 mol/l, $0,5 \mathrm{~mol} / \mathrm{l}$ und $1,0 \mathrm{~mol} / \mathrm{l}$.

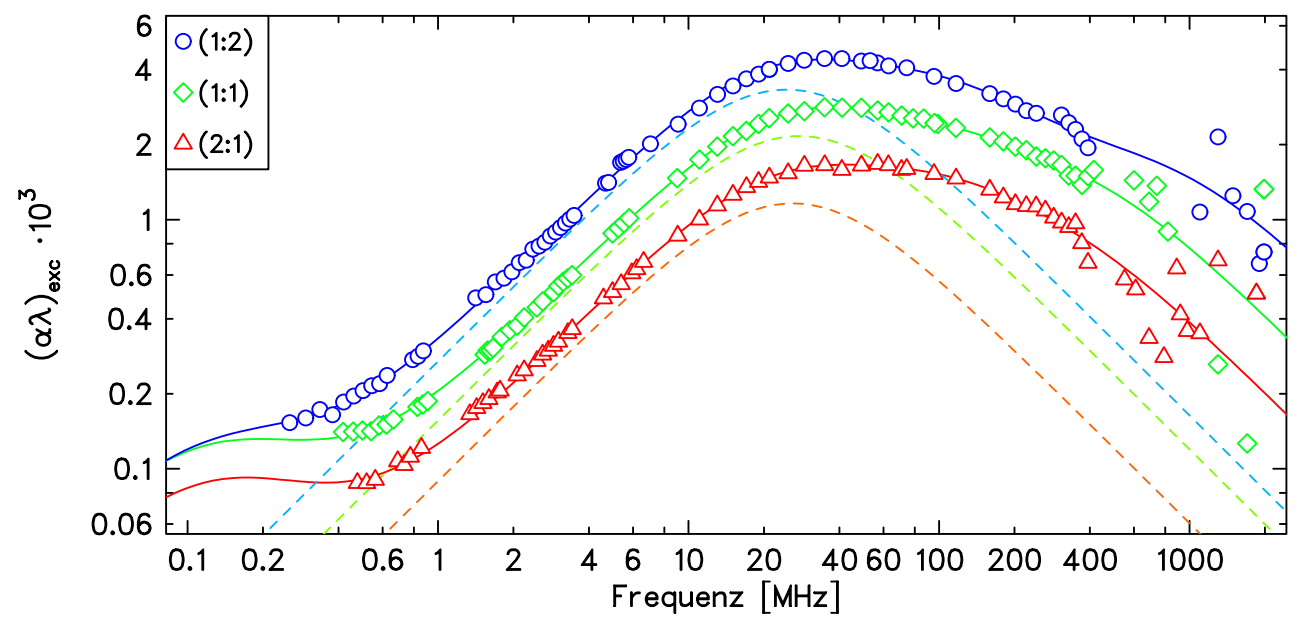

Abbildung 7.12: Exzess-Absorption von 0,5-molarer wässriger Fructoselösung bei $25^{\circ} \mathrm{C}$ mit Zusatz von $0,25 \mathrm{~mol} / \mathrm{l}(\triangle), 0,5 \mathrm{~mol} / \mathrm{l}(\diamond)$ und $1,0 \mathrm{~mol} / \mathrm{l}(\circ) \mathrm{CaCl}_{2}$. Dargestellt sind die Messdaten, der zusätzliche "Calcium“-Debye-Term (--) und die Summe aus vier Debye-Termen $(-)$. 


\begin{tabular}{|}
\begin{tabular}{|c|c|c|c|c|c|c|c|c|c|}
\hline $\begin{array}{c}\mathrm{c}_{\mathrm{Ca}^{2+}} \\
{[\mathrm{mol} / \mathrm{l}]}\end{array}$ & $\begin{array}{c}\tau_{1} \\
{[\mathrm{~ns}]}\end{array}$ & $\begin{array}{c}\mathrm{A}_{1} \\
{\left[10^{-3}\right]}\end{array}$ & $\begin{array}{c}\tau_{2} \\
{[\mathrm{~ns}]}\end{array}$ & $\begin{array}{c}\mathrm{A}_{2} \\
{\left[10^{-3}\right]}\end{array}$ & $\begin{array}{c}\tau_{3} \\
{[\mathrm{~ns}]}\end{array}$ & $\begin{array}{c}\mathrm{A}_{3} \\
{\left[10^{-3}\right]}\end{array}$ & $\begin{array}{c}\tau_{4} \\
{[\mathrm{~ns}]}\end{array}$ & $\begin{array}{c}\mathrm{A}_{4} \\
{\left[10^{-3}\right]}\end{array}$ & $\begin{array}{c}\mathrm{B} \\
{[\mathrm{ps}]}\end{array}$ \\
\hline 0,25 & $1194(*)$ & $0,15(1)$ & $6,1(1)$ & $2,3(1)$ & $1,49(*)$ & $1,7(2)$ & $0,46(19)$ & $0,49(1)$ & $37,0(1)$ \\
0,5 & $1194(*)$ & $0,21(1)$ & $5,8(1)$ & $4,3(1)$ & $1,49(*)$ & $2,0(2)$ & $0,42(8)$ & $1,31(11)$ & $37,7(1)$ \\
1,0 & $1194(*)$ & $0,19(1)$ & $6,5(1)$ & $6,7(1)$ & $1,49(*)$ & $4,0(2)$ & $0,20(15)$ & $1,8(14)$ & $40,9(6)$ \\
\hline
\end{tabular} \\
\hline \\
\hline
\end{tabular}

Tabelle 7.8: Parameter der Anpassung von vier Debye-Termen an die Absorptionsspektren von 0,5-molarer wässriger Fructoselösung mit Calciumchloridzusatz. Zum Vergleich sind die Parameter der reinen Fructose ebenfalls aufgeführt [16]. Die mit einem * gekennzeichneten Parameter wurden bei der Anpassung festgehalten. Die Rohdaten der Fructose/Calcium-Messungen stammen von Maximilian Schach und Kerstin Fuchs. 


\section{Diskussion}

\subsection{Disaccharid-Spektren}

\subsubsection{Maltose}

Im Abschnitt 7.1.1 wurde bereits angesprochen, dass das UltraschallabsorptionsSpektrum der 0,5-molaren wässrigen Maltoselösung nicht wie bei der 1-molaren Glucose durch zwei Debye-Terme beschrieben werden kann. Ein Disaccharid ist mithin nicht nur ein doppeltes Monosaccharid, sondern die Bindung zwischen den beiden Monosaccharidringen hat einen merklichen Einfluss auf das dynamische Verhalten des gesamten Moleküls. Diese Aussage wird durch die Spektren der weiteren untersuchten Disaccharide gestützt. Auch bei Trehalose, Lactose und Sucrose sind die gemessen Spektren nicht als Summe derer der Einzelkomponenten zu beschreiben. Ein direkter Vergleich der Maltose- und Glucose-Spektren zeigt bei der 0,5-molaren wässrigen Maltoselösung bei $25^{\circ} \mathrm{C}$ einen zusätzlichen Relaxationsterm mit einer Zeitkonstanten von ca. 6 ns, der jedoch nicht durch ein Minimum im $(\alpha \lambda)_{\text {exc }}$-Verlauf vom benachbarten Term bei ca. 1,5 ns getrennt ist. Auffällig beim Vergleich der Spektren ist hingegen die Übereinstimmung der Relaxationszeiten der beiden hochfrequenten Debye-Terme. Deren molekulare Ursachen sollen im Folgenden näher untersucht werden.

\subsection{Rotationsisomerie der exocyclischen Hydroxymethyl-Gruppe}

Neben dem Maltosespektrum zeigen auch die Spektren der Disaccharide Trehalose, Lactose und Sucrose eine Relaxation mit einer Zeitkonstanten $\tau$ im Bereich von 0,8-2 ns. Eine Relaxation im gleichen Zeitbereich ist ebenfalls bei einigen Monosacchariden zu beobachten, die eine exocyclische Hydroxymethyl-Gruppe besitzen, wie z.B. die Glucopyranose mit dem sechsten Kohlenstoffatom. Bei den Monosacchariden wurde sie einem Wechsel zwischen den $\omega$-Rotameren gg, gt und tg, mithin einer Rotation der exocyclischen Gruppe, zugeordnet [21][56][16].

\section{Glucose und Galactose}

Die betrachteten Disaccharide enthalten mit der Glucose und Galactose zwei Monosaccharide in Pyranoseform, die jeweils eine exocyclische Hydroxymethyl-Gruppe besitzen. Betrachtet man jedoch die in Abbildung 8.2 dargestellten Spektren der Ultraschallzusatzabsorption längs einer Wellenlänge dieser Zucker, so zeigen sich merkliche Unterschiede, insbesondere gibt es bei der Galactose nur noch einen hochfrequenten Prozess, der in der Relaxationszeit genau zwischen den beiden der Glucose liegt. Im Falle der Glucose lässt sich die Relaxationsstufe bei $\tau \approx 2$ ns der 


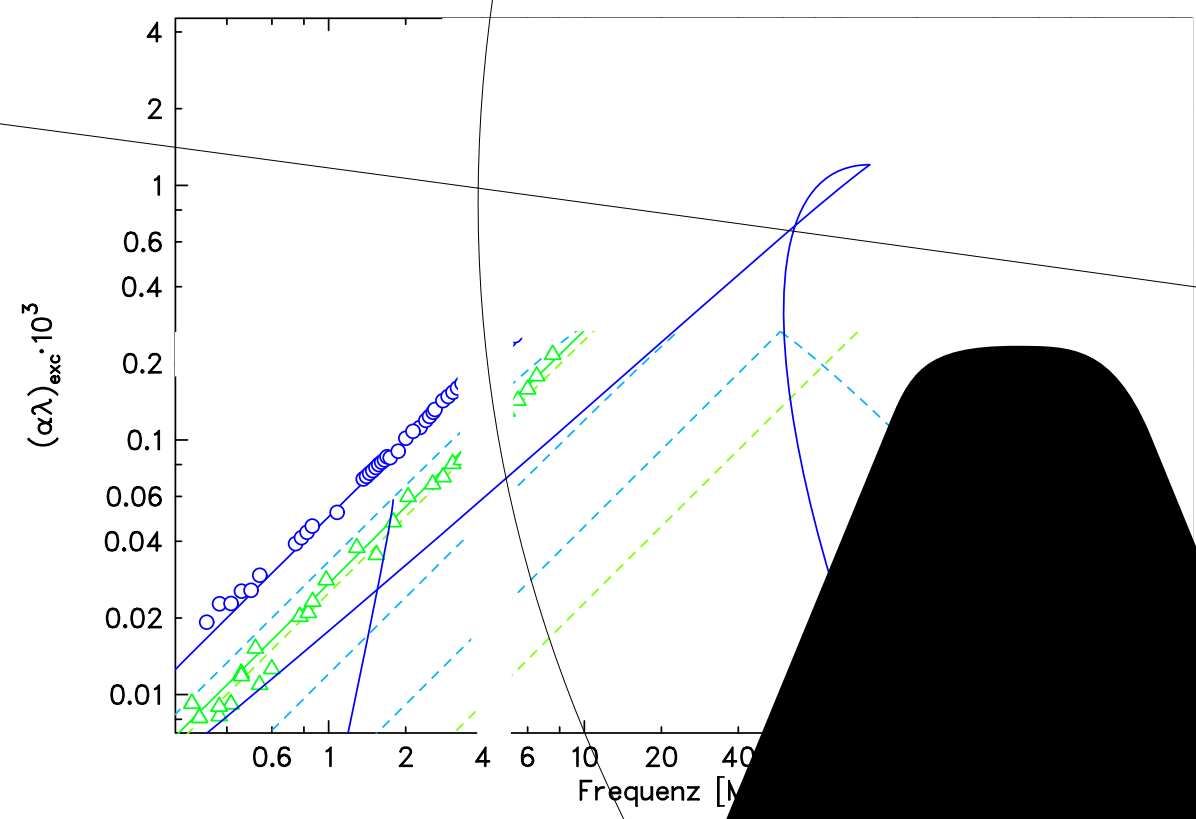


Frage, warum bei der Galactose einer der beiden Prozesse nicht mehr im Ultraschallspektrum erscheint. Der vermeintlich nur geringe Unterschied zwischen Glucose und Galactose ist die Stellung der Hydroxy-Gruppe am vierten Kohlenstoffatom. Bei der Glucose ist diese äquatorial ausgerichtet, bei der Galactose axial (siehe Abbildung 6.3). Dieser Wechsel der Stellung der OH-Gruppe hat jedoch grundlegenden Einfluss auf die Rotamerverteilung der exocyclischen Gruppe, da deren Hydroxy-Gruppe unmittelbar mit derjenigen am vierten Kohlenstoffatom wechselwirken kann. Bereits anschaulich mit Hilfe der Standardatomradien im Kalottenmodell wird deutlich, dass eine direkte Nachbarschaft der $\mathrm{C}_{4}-$ und $\mathrm{C}_{6}-\mathrm{OH}-$ Gruppen sterisch ungünstig ist. Aus Molekulardynamik-Rechnungen nach Kirschner und Woods [64] bzw. Eklund und Widmalm [65] ergibt sich, dass bei Methyl- $\alpha-$ DGalactopyranose die gg-Rotamerkonformation im Abstoßungspotential energetisch ungünstig ist, während die gt-- und tg-Konformation durch eine relativ kleine Aktivierungsenergiebariere getrennt sind (siehe Abbildung 8.3, zum Vergleich, bei $25^{\circ} \mathrm{C}$ beträgt die thermische Energie $R T \approx 2,5 \mathrm{~kJ} / \mathrm{mol}$ ). Die Reaktionsenthalpie ist kleiner als $1 \mathrm{kcal} / \mathrm{mol}$. Bei der Glucose (bzw. dem Methyl-Glucopyranosid) hingegen ist die tg-Konformation energetisch benachteiligt, die gt- und die gg-Konformationen sind im Abstoßungspotential durch eine Aktivierungsenergie von ca. 29 kJ/mol getrennt. Die Anziehungspotentiale aus entsprechenden quantenmechanischen Rechnungen ergeben nach Kirschner/Woods [64] die gleiche Stabilität aller drei Rotamere und liefern mithin keinen Beitrag zur Reaktionsenthalpie.

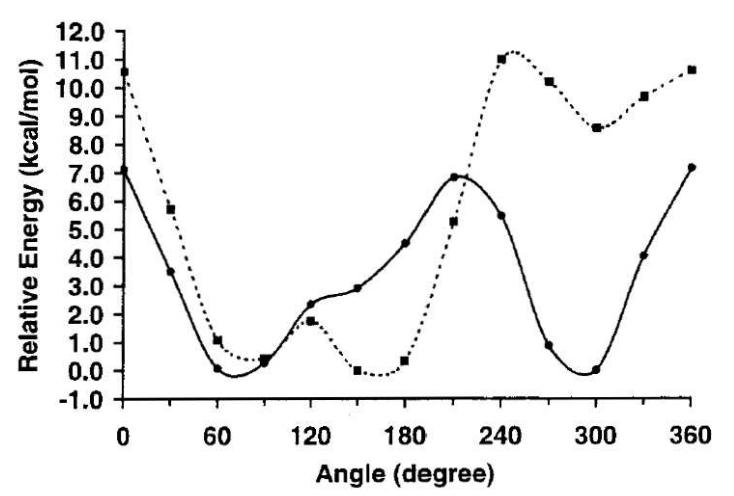

\begin{abstract}
Abbildung 8.3: Abstoßendes Potential der Rotation um $\omega$ für Methyl- $\alpha-$ D-Galactopyranosid (--) und Methyl- $\alpha-$ D-Glucopyranosid (-). Molekulardynamik Simulation nach Kirschner und Woods [64].
\end{abstract}

\title{
Relaxationszeit der $\omega$-Rotation bei Glucose
}

Bei der Rotation der exocyclischen Gruppe der Glucose liegt mithin ein Konformationsgleichgewicht mit zwei diskreten Zuständen vor.

$$
\operatorname{gg} \underset{k_{b}}{\stackrel{k_{f}}{\rightleftharpoons}} \operatorname{gt}
$$


Hierbei sind $k_{f}$ und $k_{b}$ die Hin- bzw. Rückreaktionsgeschwindigkeit. Für eine solche Konformationsumwandlung eines Moleküls ist im Ultraschallspektrum eine Einzelzeitrelaxation zu erwarten, deren Relaxationszeit nicht von der Konzentration des Kohlenhydrats abhängig ist [41]:

$$
\tau^{-1}=k_{f}+k_{b}
$$

Konzentrationsabhängige Ultraschallabsorptionsmessungen an Glucoselösungen von Behrends und Riech [21] mit insgesamt vier Konzentrationen von 0,3 mol/l bis $1,0 \mathrm{~mol} / 1$ zeigen im Rahmen der Messfehler eine konstante Relaxationszeit mit einem Mittelwert von $\tau=2,0(2)$ ns. Die Rotamerverteilung und Übergangsraten der Computersimulationen sowohl von Kirschner/Woods (vgl. Abbildung 8.4) wie auch Eklund/Widmalm erlauben eine Abschätzung der Relaxationszeiten im Bereich einiger Nanosekunden. Diese stimmen gut mit den genannten Messergebnissen an Glucose wie auch Methyl $\alpha-\mathrm{D}$-Glucopyranosid [56] überein. Somit ist zu erwarten, dass auch bei den Disacchariden, die Glucopyranose als zumindest einen Konstituenten enthalten, ein solcher Relaxationsprozess auftritt.
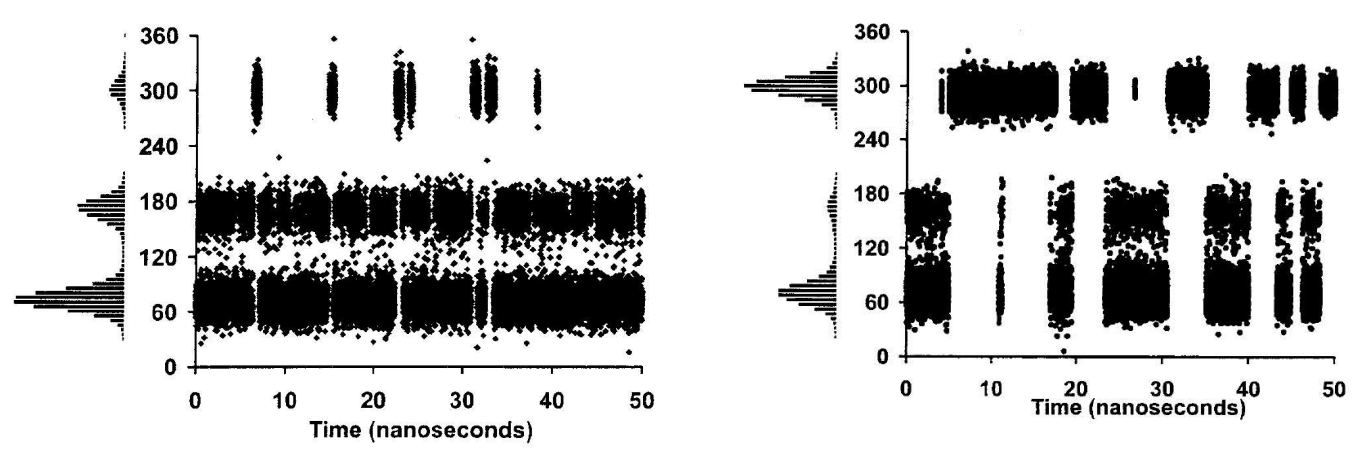

\begin{abstract}
Abbildung 8.4: 50 ns Trajektorie der Rotation um $\omega$ für Methyl- $\alpha-\mathrm{D}-$ Galactopyranosid (links) und Methyl- $\alpha-\mathrm{D}-$ Glucopyranosid (rechts). Molekulardynamik Simulation nach Kirschner und Woods [64].
\end{abstract}

\title{
Relaxationsamplitude, Reaktionsvolumina
}

Weiterhin geht aus den mit NMR-Methoden gemessenen Rotamerverteilungen und den Simulationsrechnungen hervor, dass die Reaktionsenthalpie zwischen den gt und gg Zuständen der Glucose $\leq 1 \mathrm{~kJ} / \mathrm{mol}$ beträgt (vgl. Tabelle 8.1). Unter Verwendung des thermischen Ausdehnungskoeffizienten und der spezifischen isobaren Wärmekapazität des Lösungsmittels Wasser $\left(\alpha_{p}=2,5890 \cdot 10^{-4} \mathrm{~K}^{-1}, C_{p}=4179,3 \mathrm{~J} / \mathrm{kg} \mathrm{K}\right.$ [78] $)$ lässt sich - als erste Näherung - der Enthalpiebeitrag zur Amplitude der DebyeFunktion nach Gleichung (2.13) mit ca. $6 \cdot 10^{-2} \mathrm{ml} / \mathrm{mol}$ abschätzen und im Vergleich zum Volumenbeitrag vernachlässigen. Legt man wieder eine Konformationsänderung zugrunde, so erhält man bei einer Rotamerverteilung nach Tabelle 8.1 und einer Gesamtkonzentration beider Rotamere von $c_{\text {ges }}$ einen Faktor $\Gamma$ [41]:

$$
\Gamma^{-1}=c_{\text {ges }}^{-1} \cdot\left(0,4^{-1}+0,6^{-1}\right) \Rightarrow \Gamma=0,24 \cdot c_{\text {ges }} .
$$


Damit kann das Reaktionsvolumen aus den gemessenen Amplituden der DebyeTerme nach Gleichung (2.13) abgeschätzt werden. Im Falle der Glucose erhält man als Reaktionsvolumen der Rotation der exocyclischen Gruppe aus Messungen bei vier Konzentrationen den sinnvollen Wert von $\Delta V=1,6(1) \mathrm{ml} / \mathrm{mol}$.

Bei der Methyl-D-Galactopyranose beträgt die Reaktionsenthalpie zwischen dem tg- bzw. gt-Rotamer und der gg-Konformation aus den quantenmechanischen Rechnungen von Kirschner/Woods etwa $5 \mathrm{kcal} / \mathrm{mol}(\approx 21 \mathrm{~kJ} / \mathrm{mol})$ [64]. Damit wird die Population der gg-Konformation sehr klein, folglich auch der $\Gamma$-Faktor und damit die Amplitude des Debye-Relaxationstermes. Auch nach Eklund/Widmalm [65] beträgt die betrachtete Reaktionsenthalpie, je nach Art der Berechnung des Potentials, mindestens $5 \mathrm{kcal} / \mathrm{mol}$. Eine Reaktionsenthalpie von $5 \mathrm{kcal} / \mathrm{mol}$ steht allerdings im Widerspruch zur gemessenen Rotamerverteilung nach Nishida (vgl. Tabelle 8.1). Da im Ultraschallspektrum der Galactose nur eine hochfrequente Relaxationsstufe auftritt, kann man zusammenfassend schließen, dass diese einem Assoziationsvorgang und nicht der Rotation der exocyclischen Gruppe zugeordnet werden kann.

\begin{tabular}{|l|c|c|c|}
\hline Saccharid & \multicolumn{3}{|c|}{ Rotamere [\%] } \\
& gt & tg & gg \\
\hline$\alpha-$ D-Glucopyranose & 44 & 0 & 56 \\
$\beta-$ D-Glucopyranose & 45 & 2 & 53 \\
Methyl- $\alpha-$ D-Glucopyranosid & 38 & 5 & 57 \\
Methyl- $\alpha-$ D-Galactopyranosid & 47 & 39 & 14 \\
\hline
\end{tabular}

Tabelle 8.1: Rotamerverteilung bei Drehung um $\omega$ aus ${ }^{1} \mathrm{H}-\mathrm{NMR}-\mathrm{Messungen}$ in $\mathrm{D}_{2} \mathrm{O}$ nach [70].

\section{Reaktionsvolumina für Disaccharide}

Wertet man mit diesem Ergebnis die Amplituden des 2 ns-Prozesses der Disaccharide aus, so erhält man die in Tabelle 8.2 gelisteten Ergebnisse der Abschätzung des Reaktionsvolumens. Bei der Glucose ist jeweils eine exocyclische HydroxymethylGruppe an der Relaxation beteiligt, bei den Disacchariden Maltose und Trehalose zwei. Bei der Lactose liefert nur die Hydroxymethyl-Gruppe des Glucoseringes einen Beitrag zum Ultraschallspektrum, diejenige der Galactopyranose hingegen nicht. Im Falle der Sucrose liegen drei Hydroxymethyl-Gruppen vor, da eine jedoch am ersten Kohlenstoffatom des Fructofuranoseringes gebunden ist, das an der Sauerstoffbrücke beteiligt ist, wurde angenommen, dass deren Rotation stark behindert ist. Da der Wert für $\Delta V$ dicht am Mittelwert von 1,35 ml/mol der Reaktionsvolumina für Glucose und Fructose $(1,1 \mathrm{ml} / \mathrm{mol}$ [16]) liegt, scheint auch diese Annahme gerechtfertigt. Als zusätzliche Untermauerung der Annahme, dass die Relaxation mit einer Zeitkonstanten von 1-2 ns der Rotation der exocyclischen Gruppe zugeordnet werden kann, dient das $(\alpha \lambda)_{\text {exc }}$-Spektrum der Melibiose. Da die Bindung der Monosaccharide über die exocyclische Gruppe der Glucopyranose erfolgt, sollte der entsprechende Debye-Term im Spektrum verschwinden. Wie der direkte Vergleich der Melibioseund Glucose-Spektren in Abbildung 8.5 zeigt, ist dies der Fall. 


\begin{tabular}{|l|c|c|c|c|c|c|c|c|}
\hline Lösung & $\begin{array}{c}\vartheta \\
{ }^{\circ} \mathrm{C}\end{array}$ & $\begin{array}{c}\mathrm{c} \\
{[\mathrm{mol} / \mathrm{l}]}\end{array}$ & $\begin{array}{c}\tau \\
{[\mathrm{ns}]}\end{array}$ & $\begin{array}{c}\mathrm{A} \\
10^{-3}\end{array}$ & $\begin{array}{c}\text { Anzahl }^{*} \\
\mathrm{CH}_{2} 0 \mathrm{H}\end{array}$ & $\begin{array}{c}\mathrm{c}_{s} \\
{[\mathrm{~m} / \mathrm{s}]}\end{array}$ & $\begin{array}{c}\rho \\
{[\mathrm{g} / \mathrm{ml}]}\end{array}$ & $\begin{array}{c}\Delta \mathrm{V} \\
{[\mathrm{ml} / \mathrm{mol}]}\end{array}$ \\
\hline Glucose & 25 & 0,3 & 2,09 & 0,57 & 1 & 1516 & 1,018 & 1,6 \\
& 25 & 0,5 & 2,1 & 0,95 & 1 & 1525,6 & 1,031 & 1,6 \\
& 25 & 0,8 & 1,75 & 1,59 & 1 & 1545 & 1,051 & 1,6 \\
& 25 & 1 & 2 & 1,99 & 1 & 1562,5 & 1,065 & 1,6 \\
\hline Maltose & 25 & 0,5 & 1,51 & 1,27 & 2 & 1550,5 & 1,063 & 1,3 \\
& 25 & 1 & 1,71 & 3,39 & 2 & 1613,7 & 1,129 & 1,4 \\
& 25 & 1,5 & 1,13 & 9,7 & 2 & 1686,5 & 1,193 & 1,8 \\
& 25 & 1,8 & 1,92 & 12,4 & 2 & 1734 & 1,232 & 1,8 \\
\cline { 2 - 8 } & 10 & 0,5 & 3,98 & 1,63 & 2 & 1509,8 & 1,067 & 1,5 \\
& 15 & 0,5 & 1,78 & 1,5 & 2 & 1525,2 & 1,066 & 1,4 \\
& 25 & 0,5 & 1,51 & 1,27 & 2 & 1550,5 & 1,063 & 1,3 \\
& 35 & 0,5 & 1,33 & 1,52 & 2 & 1569,2 & 1,059 & 1,4 \\
\hline Trehalose & 10 & 1 & 2,1 & 3,91 & 2 & 1594,5 & 1,140 & 1,5 \\
& 15 & 1 & 1,35 & 3,66 & 2 & 1606,7 & 1,139 & 1,4 \\
& 25 & 1 & 1,27 & 2,96 & 2 & 1625,6 & 1,136 & 1,3 \\
& 35 & 1 & 0,9 & 2,95 & 2 & 1640,5 & 1,130 & 1,3 \\
\hline Lactose & 18 & 0,5 & 1,01 & 0,63 & 1 & 1538,7 & 1,898 & 1,0 \\
& 25 & 0,5 & 1,09 & 0,65 & 1 & 1556,6 & 1,549 & 1,1 \\
& 35 & 0,5 & 1,14 & 0,56 & 1 & 1574,1 & 1,215 & 1,1 \\
\hline Sucrose & 25 & 1 & 0,83 & 2,76 & 2 & 1608,1 & 1,1295 & 1,3 \\
\hline
\end{tabular}

Tabelle 8.2: Reaktionsvolumina der Rotation der exocyclischen Gruppe für einige Mono- und Disaccharide bei verschiedenen Konzentrationen und Temperaturen. *: Anzahl der an der Relaxation beteiligten exocyclischen Hydroxymethyl-Gruppen pro Molekül (vgl. Text). Die Messdaten der Glucoselösungen stammen von Behrends und Riech 21 .

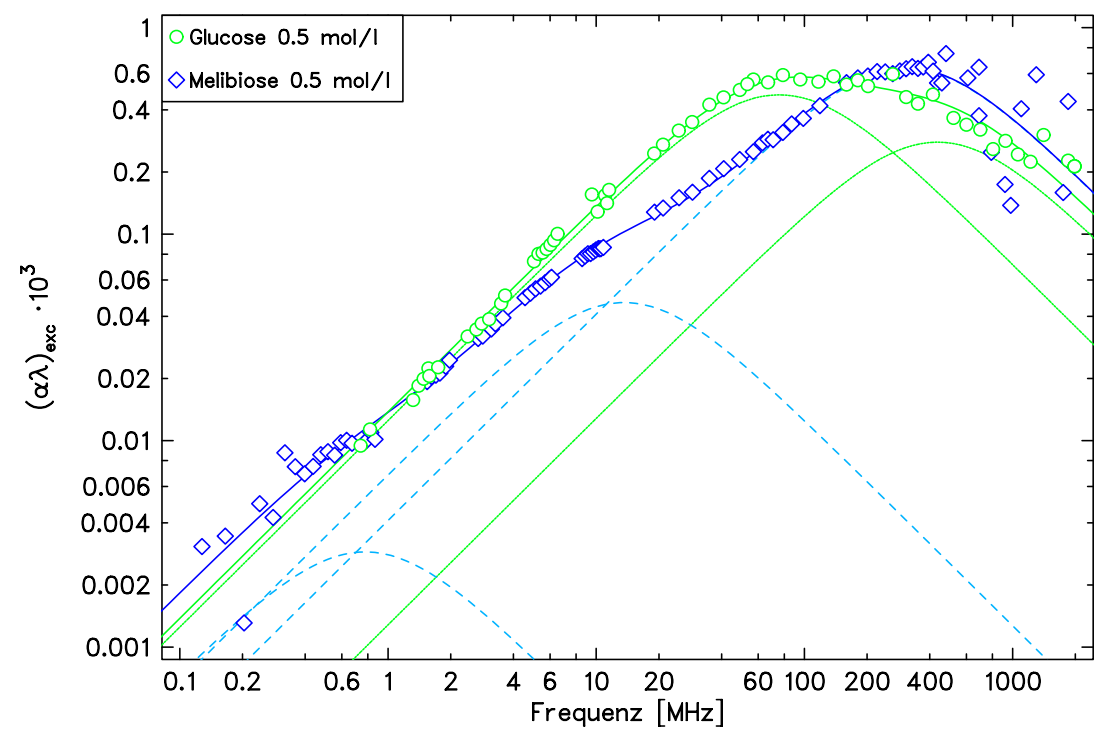

Abbildung 8.5: $(\alpha \lambda)_{\text {exc }}$-Darstellung der Spektren von 0,5-molarer wässriger Melibiose- und Glucoselösung. Die gestrichelten Linien stellen jeweils die einzelnen Debye-Terme dar, die durchgezogenen Linien deren Summe. Die Messung an Glucose stammt von Riech [21]. 


\subsection{Der „Disaccharid“ Prozess}

Zur weiteren Charakterisierung der tieffrequenten Relaxationsstufe der 0,5-molaren wässrigen Maltoselösung wurden an der Maltose konzentrations- und temperaturabhängige Messungen ausgeführt. Abbildung 8.6 zeigt den Verlauf der Relaxationszeit und der Amplitude über der Konzentration. Der Verlauf der Amplitude
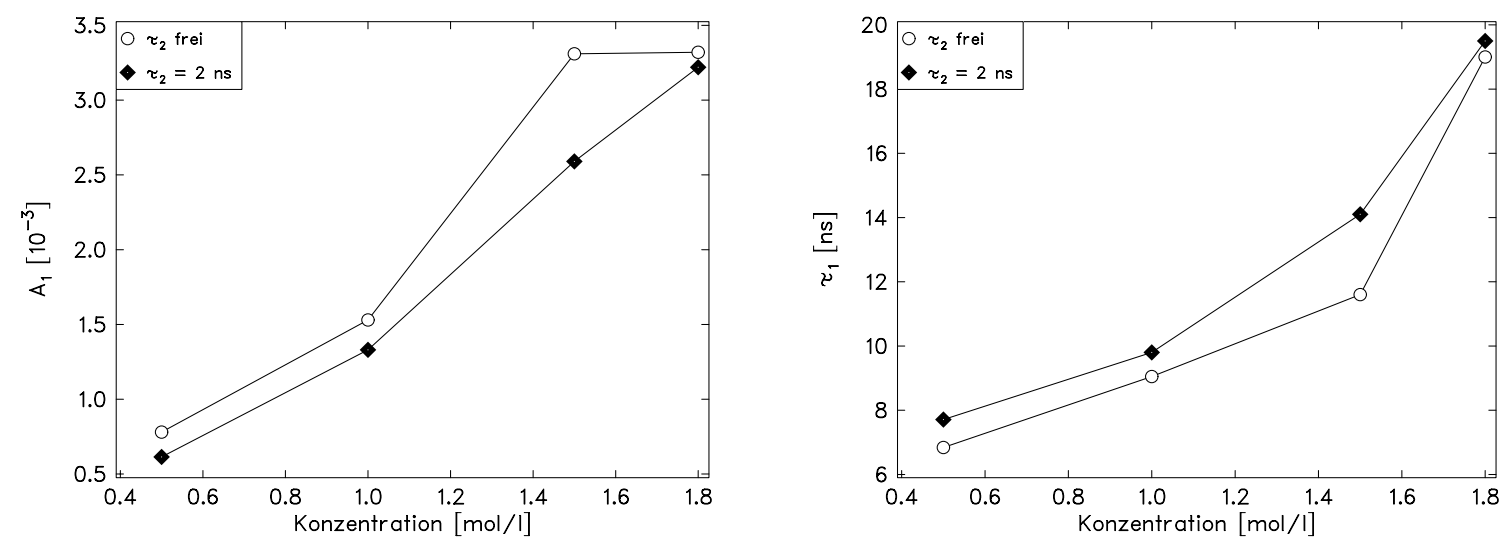

Abbildung 8.6: Verlauf der Amplitude und Relaxationszeit des tieffrequenten Debye-Spektralterms der wässrigen Maltoselösungen über der Konzentration bei jeweils $25^{\circ} \mathrm{C}$ (vgl. Tabelle 7.1).

entspricht dem zu erwartenden Verhalten, dass die Erhöhung der Anzahl der an der Relaxation beteiligten Moleküle auch die Gesamtabsorption vergrößert. Die Zunahme der Relaxationszeit mit der Konzentration ist allerdings ungewöhnlich. Im Falle einer Konformationsumwandlung sollte, wie oben angesprochen, die Relaxationszeit $\tau$ konstant bleiben, für Begegnungsprozesse, z.B. eine Dimerisierung, ist eine Abnahme von $\tau$ bei einer Konzentrationserhöhung $\left(c_{M}\right)$ zu erwarten [41]:

$$
M+M \underset{k_{b}}{\stackrel{k_{f}}{\rightleftharpoons}} \quad M_{2} \Rightarrow \tau^{-1}=4 k_{f} c_{M}+k_{b}
$$

Eine mögliche Erklärung für den Anstieg der Relaxationszeit lässt sich aus einem Vergleich mit dem Verlauf der Scherviskosität, der in Abbildung 8.7 dargestellt ist, gewinnen. Eine solche Korrelation zwischen der Zunahme der Relaxationszeit und der Viskosität über der Konzentration wurde auch bei der Dimerisierung von Essigsäure in wässrigen Lösungen beobachtet [71]. Es ist zu vermuten, dass die molekularen Ursachen, die eine Erhöhung der makroskopischen Viskosität bewirken, sich ebenfalls in der Verlangsamung der Relaxation äußern. Allerdings ist der Anstieg der Viskosität über der Konzentration größer als derjenige der Relaxationszeit. Dabei ist jedoch zu beachten, dass die Viskosität eine makroskopische Größe der Kontinuumsmechanik darstellt, während sich die Relaxationszeit auf einen molekularen Prozess bezieht. Abweichungen zwischen der makroskopischen Viskosität und der „Mikroviskosität“, die in molekularen Größenordnungen eine Rolle spielt, äußern sich z.B. auch in Lichtstreumessungen. Hierbei ist die Fragestellung interessant, ob der molekulare Prozess, der der gemessenen Relaxation zugrundeliegt, seinerseits einen Anteil an der makroskopischen Viskosität hat. Zur Beantwortung dieser Frage wurden von Behrends frequenzabhängige Messungen der Scherviskosität von 1,5-molarer wässriger 


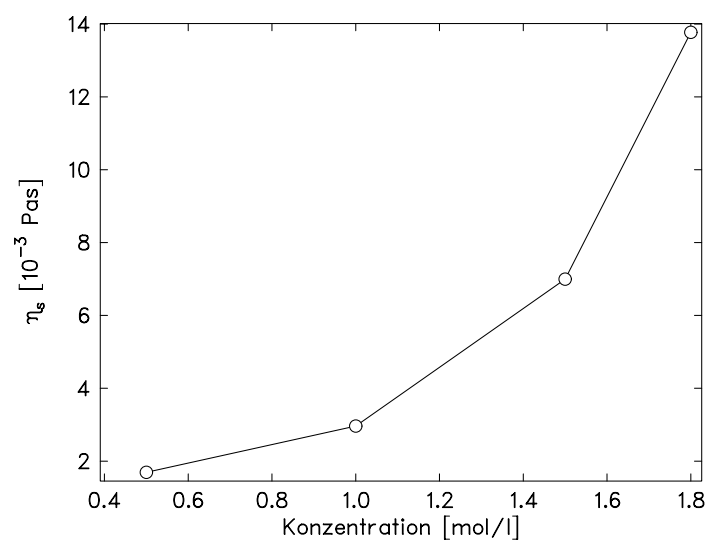

Abbildung 8.7: Konzentrationsverlauf der Scherviskosität wässriger Maltoselösungen bei $25^{\circ} \mathrm{C}$. Es handelt sich um die makroskopische, quasi-statische Scherviskosität, die mittels eines Kugelfallviskosimeters gemessen wurde (vgl. Tabelle 4.2).

Maltoselösung mittels eines Scherresonators durchgeführt [73][72]. Die Abbildung 8.8 zeigt, dass keine Relaxation der Scherviskosität im entsprechenden Frequenzbereich von ca. $6 \mathrm{MHz}$ bis $125 \mathrm{MHz}$ vorliegt. Die Abweichung des Mittelwertes der frequenzabhängigen Messung von $7,5 \cdot 10^{-3}$ Pas gegenüber der quasi statischen Messung mit dem Kugelfallviskosimeter von $7 \cdot 10^{-3}$ Pas lässt sich auf leichte Unterschiede in der Messtemperatur und der Konzentration zurückführen. Diese Abweichung hat jedoch keine signifikanten Auswirkungen auf die Aussagen zum Fehlen einer Scherviskositätsrelaxation. Insgesamt lässt sich schließen, dass die „Mikroviskosität“ zwar einen Einfluss auf die Relaxationszeit des Ultraschallabsorptionsterms hat, der zugrundeliegende Prozess jedoch seinerseits nicht zur Gesamtviskosität beiträgt. Anderenfalls müßte bei Relaxation des Ultraschall-Absorptionskoeffizienten auch die Scherviskosität relaxieren.

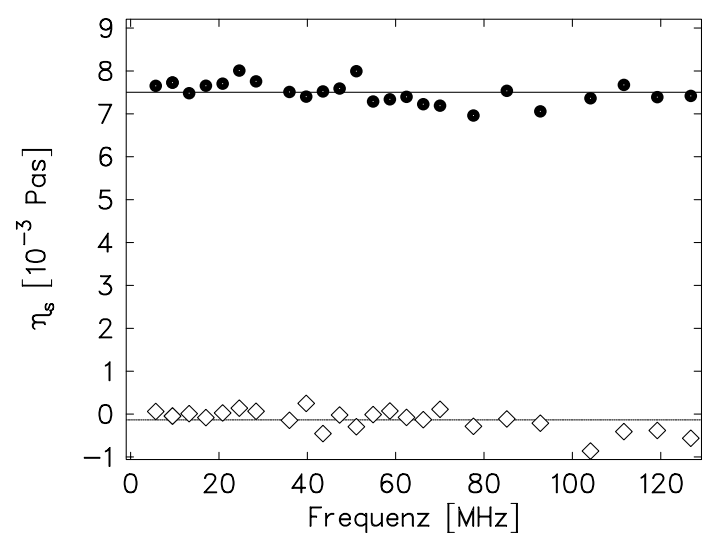

Abbildung 8.8: Frequenzverlauf der Scherviskosität einer 1,5-molaren wässrigen Maltoselösungen bei $25^{\circ} \mathrm{C}$. Aufgetragen sind der Real- $(\bullet)$ und Imaginärteil $(\diamond)$ der Scherviskosität, sowie die jeweiligen Mittelwerte.

Im Falle der Maltose, in der die Glucopyranosen aufgrund der $\alpha(1-4)-$ Bindung 
nicht mehr identisch sind, wäre als Ursache dieser Relaxation eine Aufspaltung der Rotation der exocyclischen Gruppe in zwei Prozesse mit unterschiedlichen Zeitkonstanten denkbar. Gegen diese Annahme spricht jedoch, dass auch bei der Trehalose eine Relaxation im gleichen Frequenzbereich auftritt, wie Abbildung $8.9 \mathrm{zu}$ entnehmen ist. Die Trehalose besteht aus zwei (1-1)-glycosidisch verknüpften $\alpha-$ Glucopyranosen. Sie ist mithin „symmetrisch“ und die glycosidischen Bindungswinkel $\phi$ und $\psi$ sind austauschbar. Auch bei der Lactose und der Sucrose ist ein solcher

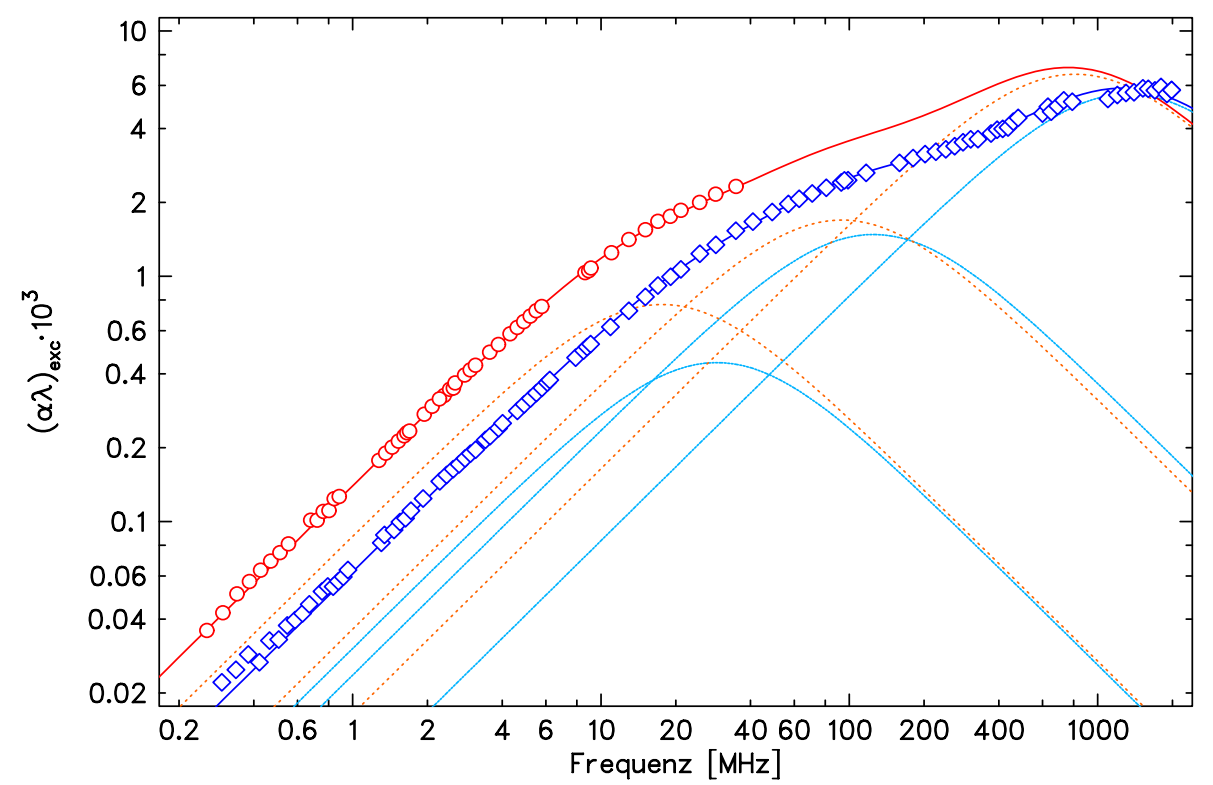




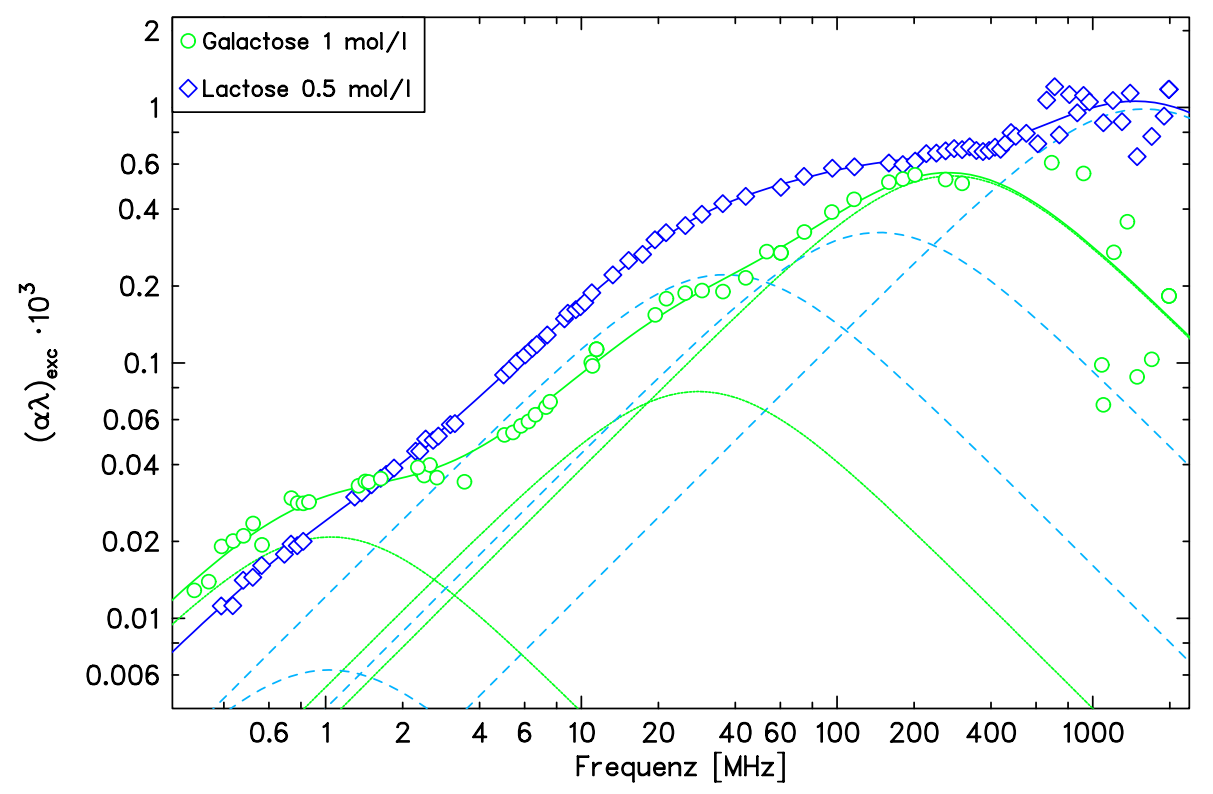

Abbildung 8.10: $(\alpha \lambda)_{\text {exc }}$-Darstellung der Spektren von 0,5-molarer wässriger Lactose- und 1,0 molarer wässriger Galactoselösung bei $25^{\circ} \mathrm{C}$.

\section{Übersicht „Disaccharid-Prozess“}

Die beiden Abbildungen 8.11 zeigen die $(\alpha \lambda)_{\text {exc }}$-Spektren der wässrigen DisaccharidLösungen mit Konzentrationen von $0,5 \mathrm{~mol} / \mathrm{l}$ und $1,0 \mathrm{~mol} / \mathrm{l}$ bei $25^{\circ} \mathrm{C}$. Der für die Disaccharide typische zusätzliche Prozess ist herausgehoben.

Aus Abbildung 6.13 ist zu entnehmen, dass das globale Minimum der Energie bei der Rotation um $\phi$ und $\psi$ für Maltose in der „syn“-Konformation vorliegt, bei der sich beide Winkel in der Nähe von $0^{\circ}$ einstellen. Daneben gibt es ein getrenntes lokales Minimum, die „anti“-Konformation, wenn $\psi$ um $180^{\circ}$ rotiert. Zwischen diesen beiden Konformationen ist ein Gleichgewicht denkbar, dass an die Ultraschallwelle koppelt, mithin als Ursache für den zusätzlichen Prozess bei Maltose in Frage käme. Allerdings zeigen die Computersimulationen von Frank [66], dass ein solcher Übergang innerhalb der Simulationszeit von $2 \mu$ s nur viermal auftritt. Somit ist die Simulationszeit immer noch viel zu kurz, um verlässliche Schlüsse über die Autokorrelationszeiten und damit die Relaxationszeiten der Ultraschallspektroskopie zu ziehen. Als Abschätzung ließe sich ein solcher Prozess unterhalb, bzw. am unteren Ende des Messfrequenzbereiches erwarten, nicht bei einer Relaxationszeit von ca. 6 ns. Wie aus der Karte der Konformationsenergien aber ebenfalls entnommen werden kann, ist das globale Minimum der „syn“-Konformation nochmals in lokale Minima unterteilt. Eine ähnlich Karte nach Lehmann [36] zeigt bis zu vier lokale Minima, Frank spricht von vier Clustern in der Computersimulation für diese Konformation. Selbst wenn eine vollständige Drehung des reduzierenden Glucoseringes vermutlich nicht als Ursache der Relaxation in Frage kommt, gibt es noch weitere mögliche Konformationsumwandlungen bei Rotation um die glycosidischen Bindungwinkel mit einer Aktivierungsenergie kleiner als $40 \mathrm{~kJ} / \mathrm{mol}$. Auch bei glycosidischen Bindungen, in denen beide Ringe in äquatorialer Position verknüpft sind, zeigen die Konformationsenergie-Karten mehrere getrennte lokale Minima. Wieder 

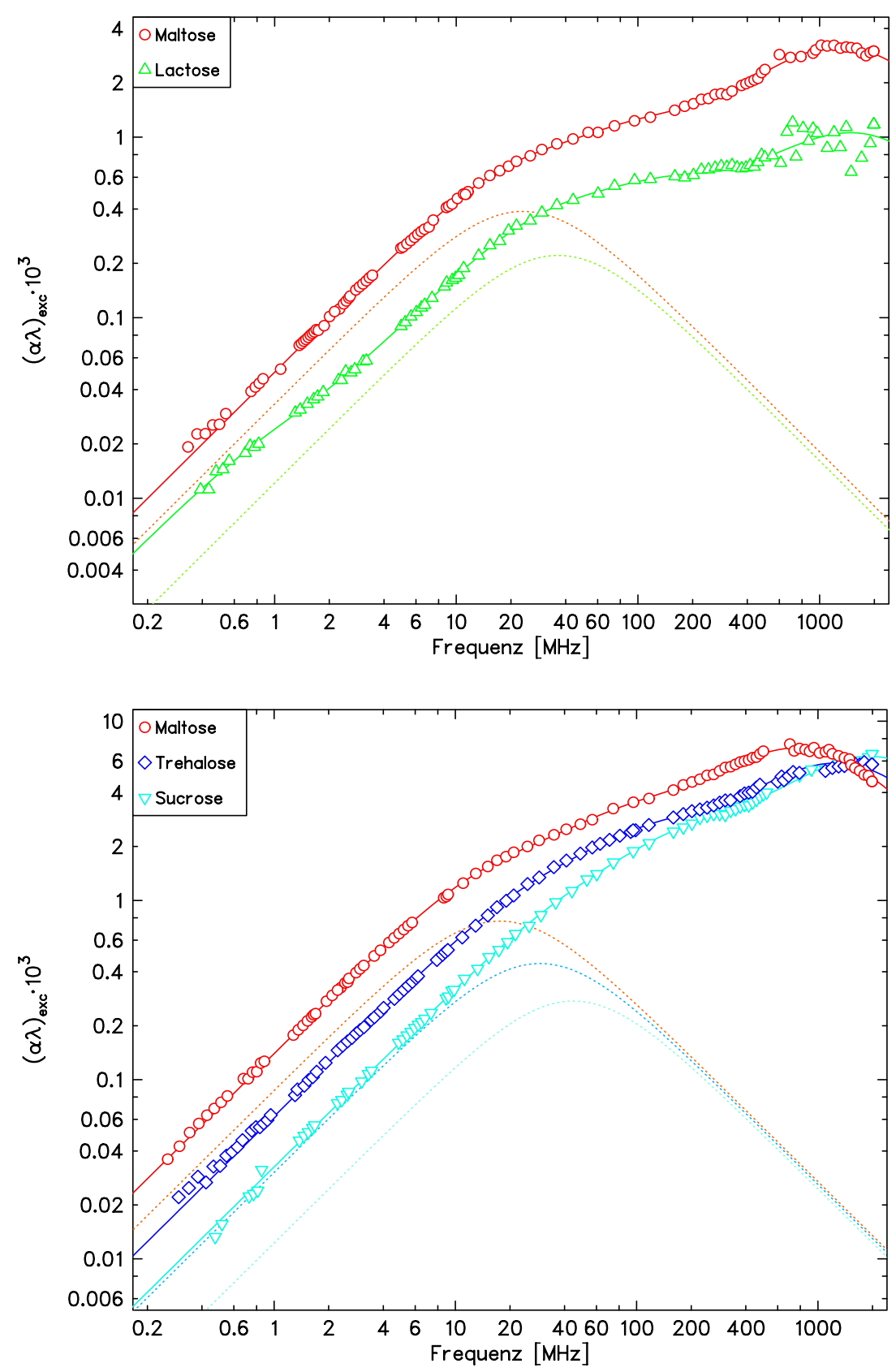

Abbildung 8.11: Zusatzabsorption wässriger 0,5-molarer Disaccharidlösungen bei $25^{\circ} \mathrm{C}$. Die gestrichelten Linien zeigen jeweils den für Disaccharide charakteristischen zusätzlichen Debye-Term, die durchgezogenen die Summe aller Spektral-Terme.

ist die Reaktionsenthalpie klein genug, dass diese Minima auch hinreichend besetzt sind. Allerdings sollte man die Energie-Karten für verschiedene Disaccharide gleichartiger glycosidischer Bindung nicht eins zu eins übertragen, wie das Beispiel der Trehalose zeigt. Diese ist, wie das $\alpha$-Galactan, axial-axial verknüpft; aufgrund der Symmetrie des Moleküls sind $\phi$ und $\psi$ austauschbar. Wie die Abbildung 6.13 zeigt, gilt dies nicht für das $\alpha$-Galactan. Als weiteres Indiz, den zusätzlichen Prozess der Disaccharide einer (teilweisen) Rotation um die glycosidischen Bindungswinkel zuzuordnen, liefert das Spektrum der Melibiose. Hier können alle gemessenen Rela- 
xationsterme den zugrundeliegenden Monosacchariden zugeordnet werden (vgl. Abbildung 8.12). Die größere Freiheit in den möglichen Konformationen aufgrund der drei nicht fixierten Einfachbindungen lässt den zusätzlichen Prozess im Ultraschallspektrum verschwinden.

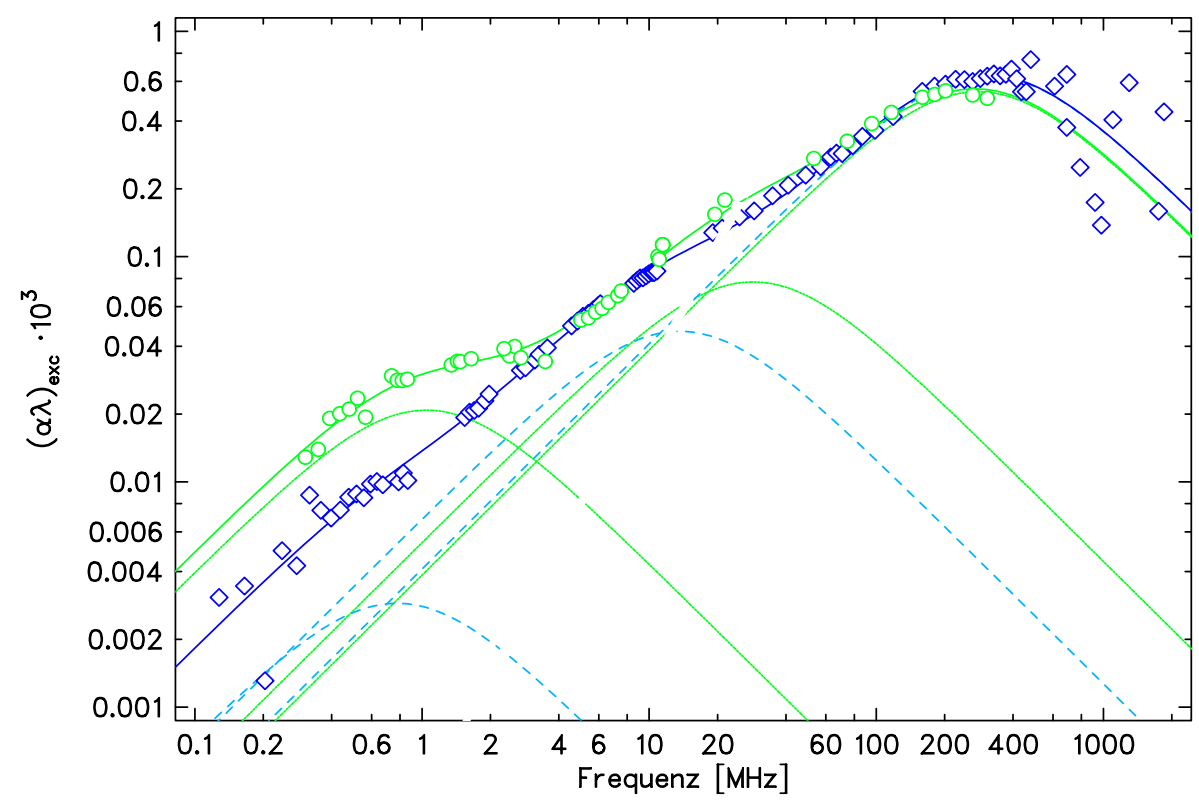




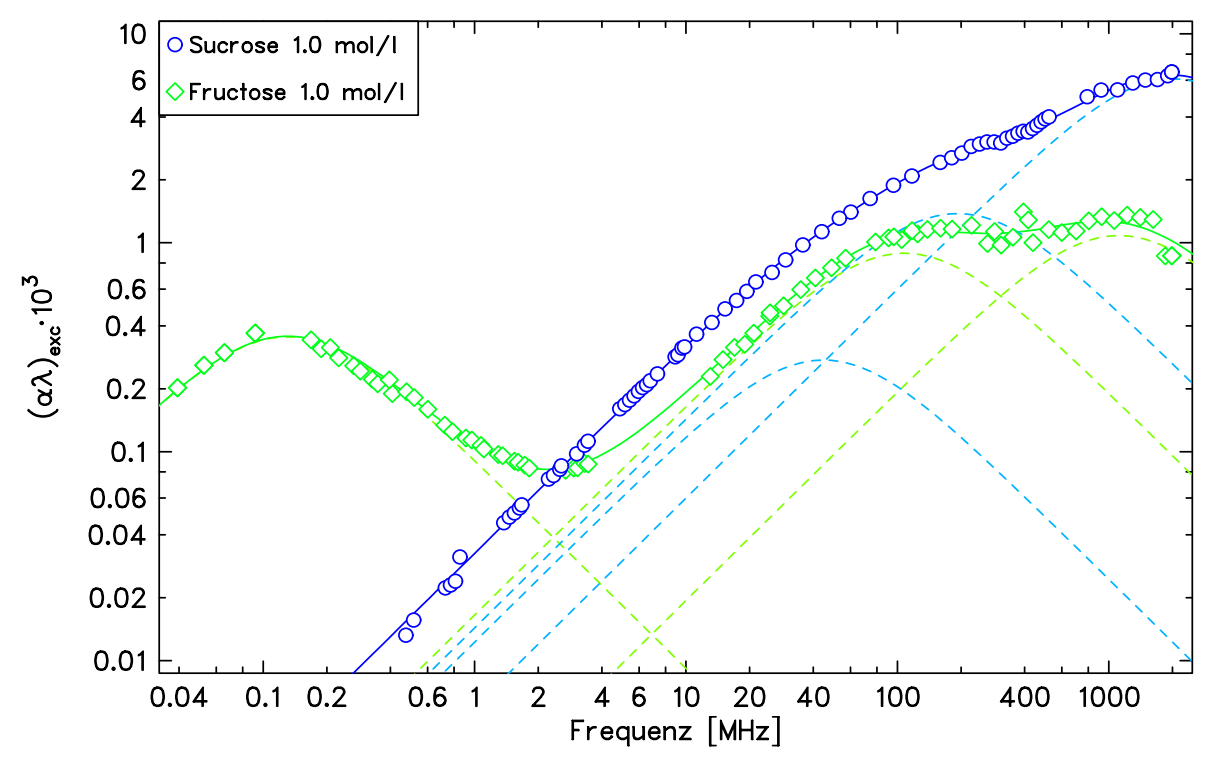

Abbildung 8.13: Zusatzabsorption von jeweils 1,0-molarer wässriger Sucrose- und Fructoselösung bei $25^{\circ} \mathrm{C}$.

stufe im Frequenzbereich von 1-2 GHz, d.h. mit einer Zeitkonstanten unter einer Nanosekunde. Diese lässt sich durch einen Debye-Prozess gut beschreiben, wobei zu beachten ist, dass dessen Parameter aufgrund der Nähe zur oberen Messfrequenzgrenze von $2 \mathrm{GHz}$ stark mit dem B-Wert der Hintergrunddämpfung und z.T. auch mit dem Prozess der exocyclischen Rotation verknüpft sind. Weiterhin streuen die Messdaten der Zusatzabsorption aufgrund des quadratischen Anstiegs der Hintergrunddämpfung auch bei guter Messgenauigkeit sehr stark. Hier erreicht die Hintergrunddämpfung mehr als $90 \%$ der Gesamtdämpfung, so dass die Exzess-Dämpfung durch Subtraktion zweier großer Zahlen berechnet wird. Zusammen bedeutet dies, dass die Parameter der Anpassung von Debye-Termen mit großen Fehlern behaftet sind, im Fall der Sucroselösung reicht dieser für die Amplitude nahezu an 100\% heran. Aus der Konzentrationsserie der Maltose ergibt sich für die Relaxationszeit der in Abbildung 8.14 dargestellte Verlauf über der Konzentration. Sowohl für die Anpassung mit freier wie auch fester Relaxationszeit der exocyclischen Rotation zeigt sich ein leichter Anstieg von $\tau_{3}$ über der Konzentration. Berücksichtig man jedoch, dass die angegebenen Fehler sich auf die konkrete Anpassung der vorliegenden Messdaten beziehen und nicht aus Mehrfachmessungen mit verschiedenen Lösungsansätzen, Befüllungen etc. gewonnen wurden, so sollte die Signifikanz dieses Anstiegs kritisch hinterfragt werden. Im Falle der Monosaccharide zeigt sich anhand konzentrationsabhängiger Messungen an Methyl- $\beta$-Xylopyranosid, dass eine Zuordnung des hochfrequenten Prozesses zu einer Assoziation der Kohlenhydrate möglich ist [56]. Eine Dimerisierung sollte nach Gleichung (8.4) mit einer Abnahme der Relaxationszeit mit zunehmender Konzentration verbunden sein. Dies ist bei Methyl- $\beta$-Xylopyranosid der Fall, nicht jedoch bei der Maltose. Die Nähe der Relaxationszeiten des hochfrequenten Prozesses bei Mono- und Disacchariden legt die Vermutung nahe, dass er durch die gleiche Reaktion hervorgerufen wird. Da die Kohlenhydrate aufgrund ihrer großen Zahl an Hydroxy-Gruppen in der Lage sind, 

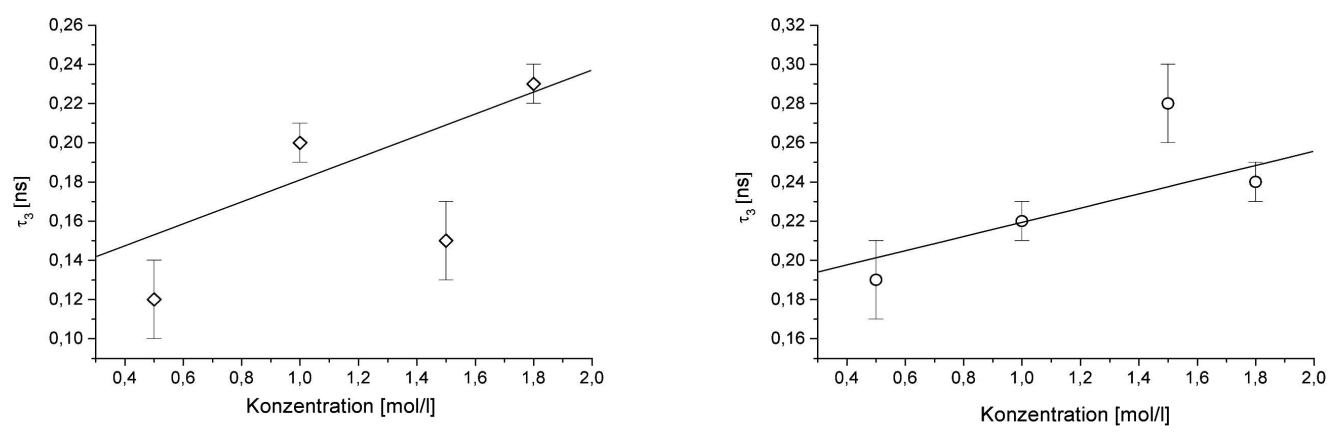

Abbildung 8.14: Verlauf der Relaxationszeit des hochfrequenten DebyeProzesses über der Konzentration für eine wässrige Maltoselösung bei $25^{\circ} \mathrm{C}$. Linke Seite: freie Anpassung von $\tau_{2}$; rechte Seite: $\tau_{2}$ auf 2 ns festgehalten. Die Relaxationszeiten und deren Fehler können Tabelle 7.1 entnommen werden. Zusätzlich ist eine mit den Fehlern der Messpunkte gewichtete Regressionsgerade eingetragen.

sich gut in das Wasserstoffbrückenbindungsnetzwerk des Wassers einzubinden und auch intramolekulare Wasserstoffbrücken bilden können, scheint eine Dimerisierung über Wasserstoffbrücken eine mögliche Erklärung des hochfrequenten Prozesses. 


\subsection{Kohlenhydrate mit Calciumzusatz}

Zusätzlich zu den reinen Disacchariden wurde im Rahmen dieser Arbeit die Wechselwirkung von Kohlenhydraten mit Calcium-Ionen weiter untersucht. Hierbei lassen sich die bisher gemessenen Kohlenhydrate in zwei Gruppen einteilen.

\subsubsection{Maltose und Glucose mit Calcium}

Bei der Untersuchung einer 0,5-molaren wässrigen Maltoselösung unter Zusatz von 0,25 mol/l, 0,5 mol/l und $1 \mathrm{~mol} / 1$ Calcium-Ionen (aus $\mathrm{CaCl}_{2}$ ) zeigt sich, dass die Relaxationszeiten aller drei Debye-Terme der reinen Maltose im Rahmen der Messfehler konstant bleiben (siehe Abbildung 8.15 und Tabelle 7.7). Die Amplituden nehmen mit zunehmender Calcium-Konzentration zu (siehe Tabelle 7.7). Im zur Verfügung stehenden Messbereich ist also kein zusätzlicher, der Wechselwirkung zwischen Zucker und Calcium zuzuordnender Prozess vorhanden. Ein ähnliches Ver-

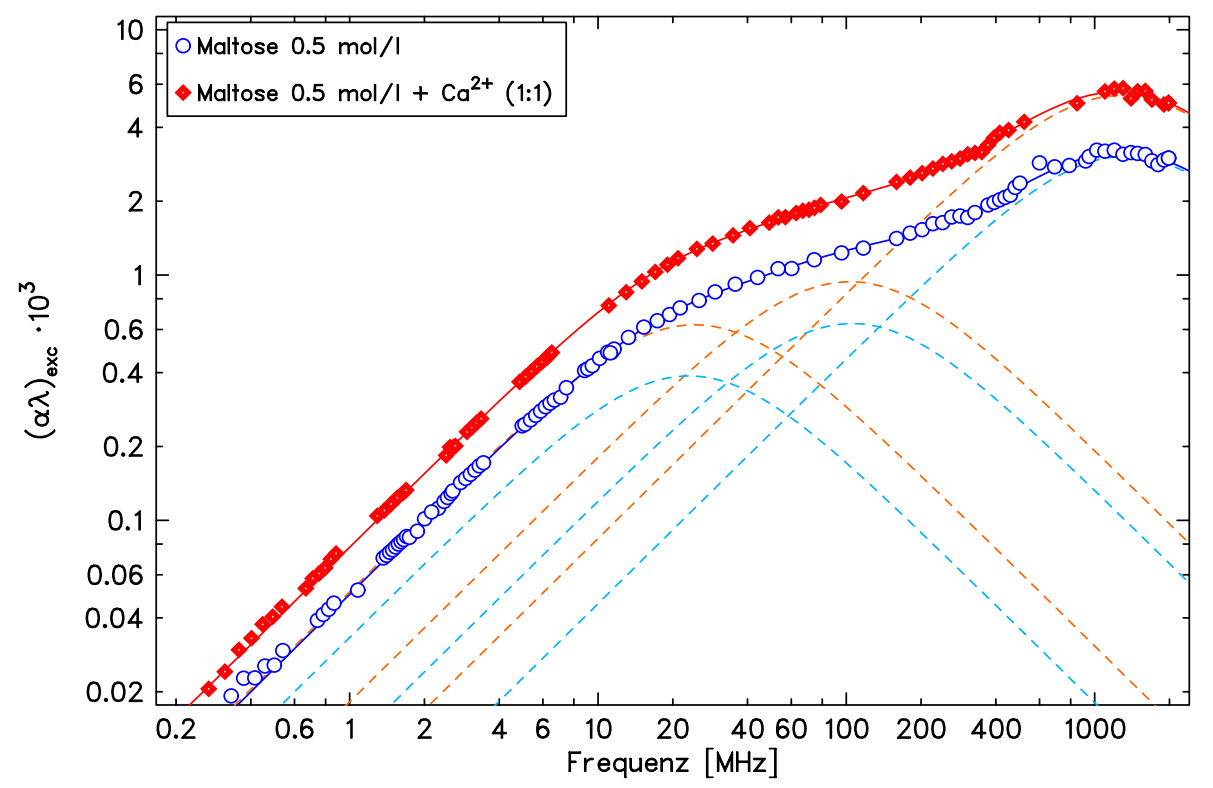

Abbildung 8.15: Exzess-Absorption von 0,5-molarer wässriger Maltoselösung bei $25^{\circ} \mathrm{C}$; (०) ohne und $(\bullet)$ mit Zusatz von $0,5 \mathrm{~mol} / 1 \mathrm{CaCl}_{2}$.

halten ist auch beim Zusatz von Calcium-Ionen zu einer wässrigen Glucoselösung zu beobachten (Abbildung 8.16). Auch hier wird kein zusätzlicher Relaxationsprozess benötigt: Die Relaxationszeiten der Debye-Terme, die auch die Spektren der reinen Glucose beschreiben, werden nicht signifikant verschoben und ihre Amplituden nehmen mit steigender Calcium-Konzentration zu. Da mit der Zugabe von jedem $\mathrm{Mol} \mathrm{CaCl}_{2}$ zu einer Kohlenhydratlösung die Konzentration an Ionen um drei Mol steigt, nimmt entsprechend der Anteil an Hydratwasser gegenüber dem freien (,bulk“) Wasser überproportional zu. Die Ionen bewirken eine Störung der relativ voluminösen Struktur des reinen Wassers und richten das Hydratwasser in der unmittelbaren Umgebung durch ihr Coulomb-Feld aus. Die als Folge veränderte gesamte Wasserstruktur der Lösungen hat ebenfalls Einfluss auf das Hydratwasser der 
Kohlenhydrate, und damit auf die effektive Volumenänderung bei der Relaxation in eine neue Gleichgewichtslage. Die Umorientierungszeit des Hydratwassers bleibt, wie dielektrische Messungen zeigen [74], hingegen weiterhin schnell gegenüber den hier betrachteten Relaxationszeiten, so dass diese nur wenig beeinflusst werden.

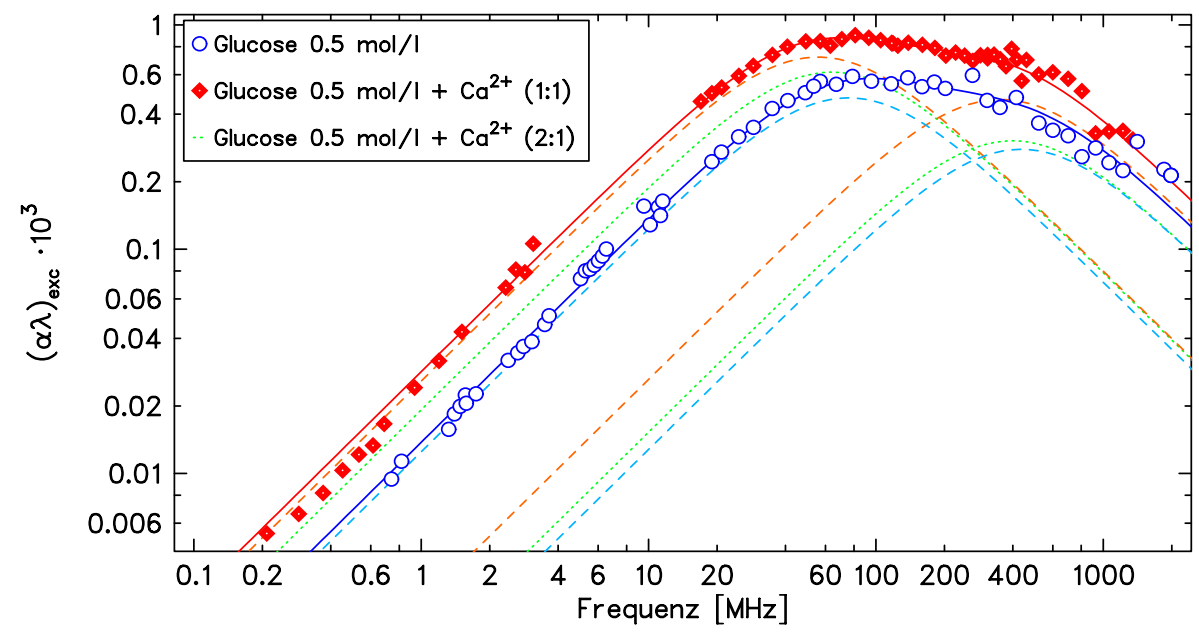

Abbildung 8.16: Exzess-Absorption von 0,5-molarer wässriger Glucoselösung bei $25^{\circ} \mathrm{C}$; ( $)$ ohne und $(\bullet)$ mit Zusatz von $0,5 \mathrm{~mol} / 1 \mathrm{CaCl}_{2}$ [21]. Die gestrichelten Linien geben jeweils die einzelnen Debye-Spektralterme, die durchgezogenen ihre Summe wieder.

\subsubsection{Fructose und Xylose}

Anders verhält es sich bei wässrigen Lösungen von Fructose und Xylose bei Zugabe von Calcium-Ionen. Interessant ist dabei insbesondere, dass im Falle der Xylose ein anderes Verhalten als bei der Glucose beobachtet wird. Der Xylose fehlt gegenüber der Glucose die exocyclische Hydroxymethyl-Gruppe, die Ausrichtung aller weiteren Hydroxy-Gruppen ist jedoch mit denen der Glucose identisch und selbst das Rotamergleichgewicht zwischen $\alpha$ - und $\beta$-Pyranose ist nahezu gleich. Der $\alpha$-Pyranose Anteil der Glucose liegt bei 38\% derjenige der Xylose bei 36,5\% [36]; die Konzentrationen der Furanosen und der acyclischen Form können mit zusammen weniger als $1 \%$ vernachlässigt werden. Wie der Abbildung $8.17 \mathrm{zu}$ entnehmen ist, treten bei der Xylose drei zusätzliche Relaxationsterme auf, die die um eine Größenordnung in der Amplitude kleineren Relaxationsstufen der Xylose-Spektren ohne Salzzusatz vollständig überlagern. Und auch bei der Fructose zeigt sich bei Zugabe von Calcium gegenüber der reinen Fructose eine sehr ausgeprägte zusätzliche Relaxationsstufe mit einer Zeitkonstanten von ca. 6 ns (vgl. Abbildung 8.18). Abbildung 8.19 zeigt den Amplitudenverlauf dieses Prozesses über der Calcium-Konzentration.

\section{Modell der Assoziation von Kohlenhydraten mit Calcium}

Betrachtet man zunächst die zusätzlichen Debye-Spektralterme, die sich aufgrund des Zusatzes von Calcium-Ionen zu wässrigen Kohlenhydratlösungen ergeben, so legen die Ergebnisse einen mehrstufigen Assoziationsmechanismus nahe. Dieser lässt 


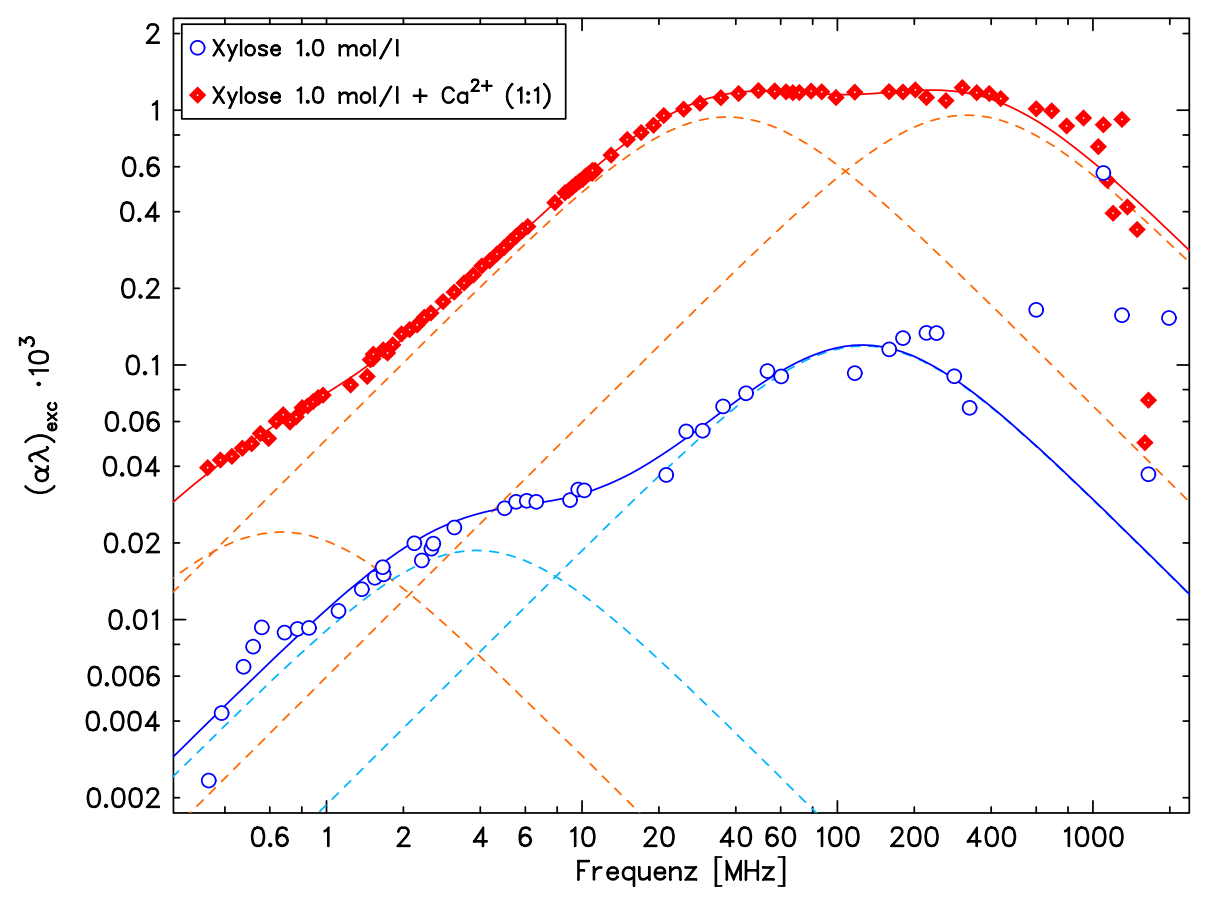

Abbildung 8.17: Exzess-Absorption von 0,5-molarer wässriger Xyloselösung bei $25^{\circ} \mathrm{C}$; (०) ohne und $(\bullet)$ mit Zusatz von $0,5 \mathrm{~mol} / \mathrm{l} \mathrm{CaCl}_{2}$ [57]. Die gestrichelten Linien zeigen die angepassten Debye-Terme, die durchgezogenen deren Summe.

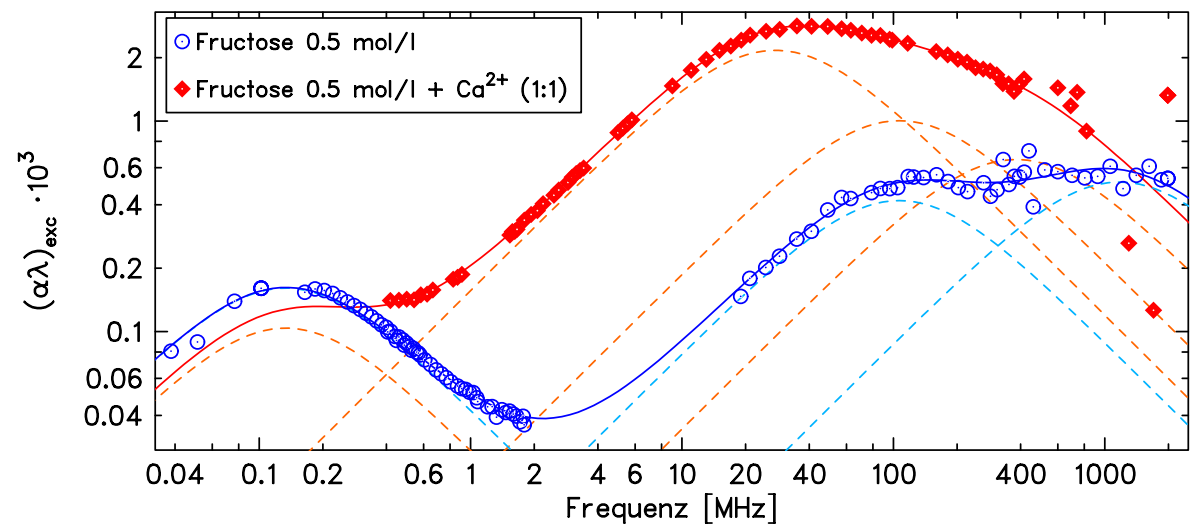

Abbildung 8.18: Exzess-Absorption von 0,5-molarer wässriger Fructoselösung bei $25^{\circ} \mathrm{C}$; (०) ohne [16] und (•) mit Zusatz von $0,5 \mathrm{~mol} / \mathrm{l} \mathrm{CaCl}_{2}$. Die durchgezogenen Linien geben die Summe aus drei bzw. vier Debye-Termen wieder, die selbst gestrichelt dargestellt sind. Die Messdaten der reinen Fructose stammen von Riech [21] und von Polacek [16]. Die Messungen an Fructose mit Calciumzusatz wurden von Kerstin Fuchs und Maximilian Schach ausgeführt.

sich in Anlehnung an die Assoziation zwei-zwei-wertiger Ionen nach dem EigenWinkler-Schema, in einen Begegnungsprozess und anschließend evtl. mehrstufigen „Umlagerungen" dieses Komplexes einteilen, die jeweils mit einer eigenen charakteristischen Zeitkonstante erfolgen. Dabei sollte die Relaxationszeit des Begegnungsprozesses, hier zweier verschiedener Moleküle, nach Strehlow [41] konzentrations- 


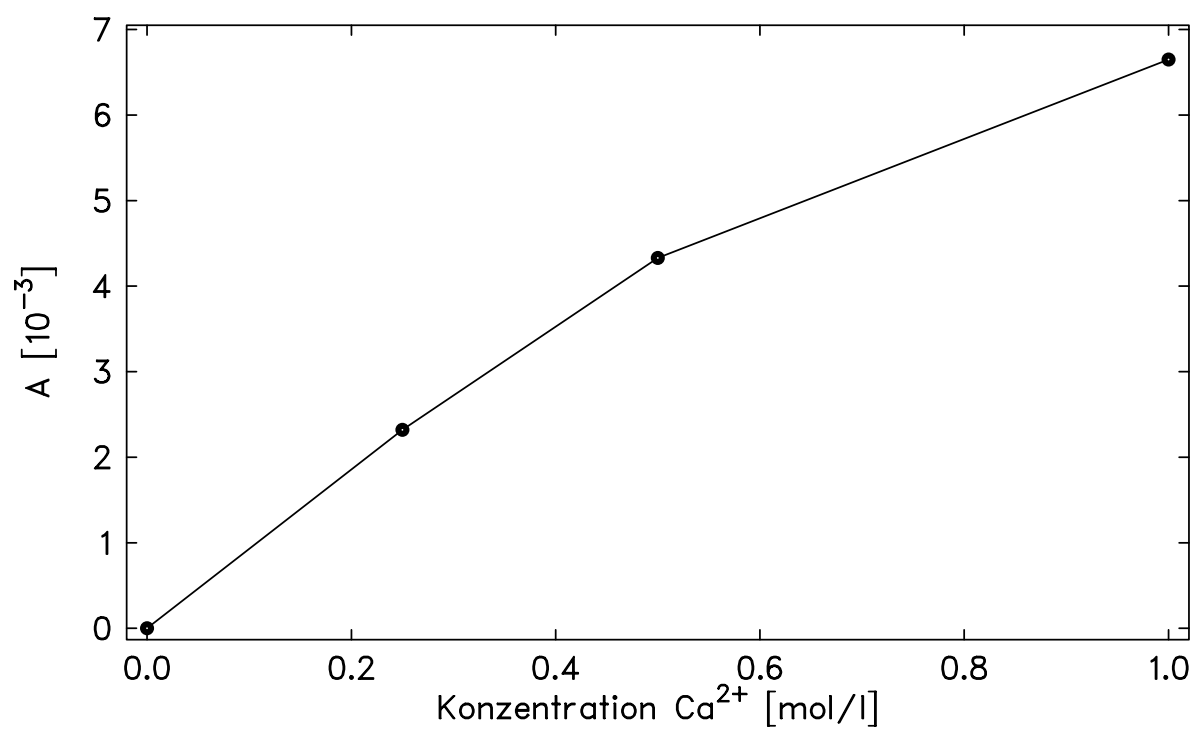

Abbildung 8.19: Verlauf der Amplitude des zusätzlichen Debye-Prozesses bei 0,5-molarer wässriger Fructoselösung über der Calcium-Konzentration bei $25^{\circ} \mathrm{C}$.

abhängig sein.

$$
A+B \underset{k_{b}}{\stackrel{k_{f}}{\rightleftharpoons}} A B \Rightarrow \tau^{-1}=4 k_{f}\left(c_{A}+c_{B}\right)+k_{b} \quad,
$$

wobei $k_{f}$ und $k_{b}$ die Hin- bzw. Rückreaktionsgeschwindigkeit bezeichnen.

Der weitere Umbau der Komplexe stellt hingegen eine unimolekulare Reaktion - quasi eine Konformationsumwandlung - dar und ist nicht mehr konzentrationsabhängig. Dies trifft auf die Relaxationsstufe bei 6 ns im Fall der Fructose bzw. 4 ns der Xylose zu.

Offen bleibt hingegen die Frage, warum sich bei Xylose, Fructose und AnhydroGlucopyranosid [58] eine zusätzliche Relaxation aufgrund der Kohlenhydrat-Calcium Wechselwirkung zeigt, während dies bei Maltose und Glucose nicht der Fall ist. Vergleicht man die Amplituden, insbesondere des Fructose-Calcium-Prozesses, mit denen der Debye-Terme der reinen Glucose und Maltose im entsprechenden Frequenzbereich, so scheint es unwahrscheinlich, dass eine hier ebenfalls vorhandene Calcium-Kohlenhydrat-Wechselwirkung durch andere Terme überdeckt wird. Offenbar reicht die bisherige Vorstellung, nach der im Falle der Pentosen und Hexosen die Pyranose-Ringe mit axial-äquatorial-axial Sequenz der Hydroxy-Gruppen und die Furanose-Ringe mit drei Hydroxy-Gruppen in cis-Stellung bevorzugt Komplexe mit Kationen bilden [77], nicht aus. Dies wird insbesondere beim Vergleich von Glucose und Xylose deutlich. Der Pyranosering beider Saccharide ist identisch, in wässriger Lösung unterscheidet sich das Verhältnis aus $\alpha$ - zu $\beta$-Pyranose nur minimal (Xylose: 35\%: 65\%, Glucose 36\%:64\%) und beide kommen nur in $\operatorname{der}{ }^{4} \mathrm{C}_{1}$ Sessel-Konformation vor [36]. Der Xylose fehlt gegenüber der Glucose „nur" die exocyclische Hydroxymethyl-Gruppe. Erstere bildet Komplexe mit $\mathrm{Ca}^{2+}$, letztere offenbar nicht. 


\subsection{Aktivierungsenthalpie}

Mit Hilfe einer „Eyring-Darstellung“ lassen sich aus dem Verlauf der Relaxationszeit über der Temperatur die Aktivierungsenthalpien der beteiligten Konformationsumwandlungen bestimmen. Dabei gilt die folgende Beziehung zwischen der mit der Ultraschallspektroskopie gemessenen Relaxationszeit und den thermodynamischen Größen [76][75]:

$$
\ln \left(\frac{\hbar \tau^{-1}}{k_{b} T}\right)=\text { const. }+\frac{\Delta H^{\sharp}}{R T}
$$

mit:

$$
\begin{aligned}
\hbar & =\mathrm{h} / 2 \pi, \mathrm{h} \text { : Plancksches Wirkungsquantum; } \\
\tau & : \text { Relaxationszeit bei der Temperatur T; } \\
k_{b} & : \text { Boltzmann Konstante; } \\
R & : \text { Gaskonstante; } \\
\Delta H^{\sharp} & : \text { Aktivierungsenthalpie. }
\end{aligned}
$$

Trägt man $\ln \left(\hbar / k_{b} T \tau\right)$ gegen $1 / T$ auf, so erhält man - unter der Annahme einer vernachlässigbaren Reaktionsenthalpie $(\Delta H=0)$ - die Aktivierungsenthalpie $\Delta H^{\sharp}$ aus der Steigung der Regressionsgeraden. Für diesen Fall $(\Delta H=0)$ sind die Aktivierungsenthalpien für Hin- und Rückreaktion gleich groß.

Aus den temperaturabhängigen Messungen an Maltose und Trehalose erhält man die in Tabelle 8.3 angegebenen Aktivierungsenthalpien für die Rotation der exocyclischen Gruppe ( $\omega$-Rotation, Relaxationszeit $\left.\tau_{2}\right)$ und für den „Disaccharid-Prozess“ (Relaxationszeit $\tau_{1}$ ).

\begin{tabular}{|l|c|c|}
\hline & „Disaccharid-Prozess “ & $\omega$-Rotation \\
\cline { 2 - 3 } Saccharid & $\Delta H^{\sharp}$ & $\begin{array}{c}\Delta H^{\sharp} \\
{[\mathrm{kJ} / \mathrm{mol}]}\end{array}$ \\
\hline Maltose & $24(8)$ & $26(12)$ \\
Trehalose & $17(3)$ & $19(5)$ \\
\hline
\end{tabular}

Tabelle 8.3: Aktivierungsenthalpien für den „Disaccharid-Prozess“ und die Rotation der exocyclischen Gruppe, jeweils berechnet aus einem „Eyring-Plot" unter Vernachlässigung der Reaktionsenthalpie.

Die Aktivierungsenthalpien beider Prozesse liegen in der Größenordnung der Energie einer Wasserstoffbrückenbindung für Wasser bei Zimmertemperatur (ca. $24 \mathrm{~kJ} / \mathrm{mol}$ [78]). Die hier gefundenen $\Delta H^{\sharp}$-Daten haben somit plausible Werte, da sich die Kohlenhydrate aufgrund ihrer Hydroxy-Gruppen gut in das Wasserstoffbrückenbindungsnetzwerk des Wassers einpassen und somit bei Konformationsänderungen zunächst bestehende $\mathrm{H}$-Brücken aufgebrochen werden müssen. Die großen Fehler der Aktivierungsenthalpien ergeben sich zum einen aus dem - auf der absoluten Temperaturskala - sehr kleinen zur Verfügung stehenden Messbereich, zum anderen durch die relative Nähe der jeweiligen Relaxationsterme zueinander. 


\section{Zusammenfassung und Ausblick}

Anhand der Spektren von Galactose und Glucose, sowie der untersuchten Disaccharide konnte gezeigt werden, dass die Einzelzeitrelaxation mit einer Relaxationszeit im Bereich von 1-2 ns der Rotation der exocyclischen Gruppe ( $\omega$-Rotation) zugeordnet werden kann. Diese Rotation tritt im Fall der Glucose und derjenigen Disaccharide, die eine Glucopyranose mit freier exocyclischer Gruppe enthalten, im Ultraschallspektrum auf und fehlt bei der Galactose aufgrund der Wechselwirkung mit der Hydroxy-Gruppe am $\mathrm{C}_{4}-$ Atom. Entsprechend sind bei der Maltose und Trehalose jeweils zwei exocyclische Gruppen an der Relaxation beteiligt, bei der Lactose eine und bei der Melibiose fehlt dieser Prozess aufgrund der (1,6)-glycosidischen Bindung zwischen der Galactopyranose und der Glucopyranose gänzlich. Diese Ergebnisse decken sich gut mit Computersimulationen von Krischner/Woods, Eklund/Widmalm und Frank, die für Methyl-Glucopyranosid bzw. Maltose eine typische Lebensdauer der $\omega$-Rotamere im Bereich einiger Nanosekunden berechnet haben.

Bei den Disacchariden gibt es, wie auch bei den Monosacchariden, einen hochfrequenten Debye-Term mit einer Relaxationsfrequenz von ca. $2 \mathrm{GHz}$, der sich mit einer Assoziation der Zuckermoleküle erklären lässt.

Zusätzlich zu den beiden oben beschriebenen Prozessen tritt bei den Disacchariden Maltose, Trehalose, Lactose und Saccharose eine weitere Einzelzeitrelaxation im Zeitbereich von ca. 6 ns auf. Diese fehlt bei den konstituierenden Monosacchariden und ist somit charakteristisch für die genannten Disaccharide. Diese sind mithin bezüglich ihres dynamischen Verhaltens nicht nur doppelte Monosaccharide und der Einfluss der glycosidischen Bindung ist im Ultraschallspektrum erkennbar. Aus Sicht der Ultraschallspektroskopie lässt sich dieser Prozess einer (teilweisen) Rotation um die glycosidischen Bindungwinkel $\omega$ und $\psi$ zuordnen, wenn die beteiligten Monosaccharideinheiten $(1,4)$ - oder $(1,1)$-glycosidisch - also über zwei nichtfixierte Einfachbindungen - miteinander verknüpft sind. Wieder verschwindet diese Relaxationsstufe im Spektrum der (1,6)-glycosidisch verknüpften Melibiose, da hier aufgrund der Brücke aus drei frei rotierenden Einfachbindungen eine wesentlich größere Konformationsvielfalt zur Verfügung steht.

Die mittels temperaturabhängiger Messungen aus „Eyring-Plots“ bestimmten Aktivierungsenthalpien sowohl der $\omega$-Rotation als auch der Rotation der monomeren Einheiten um $\phi$ und $\psi$ liegen im Bereich der Energie einer Wasserstoffbrückenbindung des Wassers. Dies ist schlüssig, da sich die Kohlenhydrate gut in das Wasserstoffbrückenbindungsnetzwerk des Wassers einpassen.

Die Untersuchungen der Wechselwirkungen von Sacchariden mit Calcium-Ionen zeigen ein stark differenziertes Verhalten bei Variation des Kohlenhydrates. Bei dem Disaccharid Maltose und der Glucose ist keine zusätzliche Relaxation messbar, die Relaxationszeiten der einzelnen Prozesse bleiben gegenüber den Lösungen ohne Cal- 
ciumzusatz konstant, die Amplituden erhöhen sich aufgrund des nun auch vorhandenen Hydratwassers der Ionen. Im Fall der Xylose und Fructose erhält man zusätzliche Relaxationsterme im Ultraschallspektrum, die auf eine zweistufige Assoziation zwischen Kohlenhydrat und Calcium hindeuten. In einem ersten schnellen Prozess nähern sich Saccharid und Calcium an; man misst eine Abnahme der Relaxationszeit bei Erhöhung der Konzentration. Es folgt ein zweiter langsamerer Prozess, dessen Relaxationszeit über der Konzentration konstant bleibt und der einer Umordnung des Komplexes zugeordnet werden kann.

Für zukünftige Arbeiten wäre zum einen eine genauere Untersuchung der Voraussetzungen interessant, die ein Saccharid erfüllen muss, damit es zu einer Wechselwirkung mit Ionen kommt, da anscheinend das einfache Modell von Angyal nicht ausreicht. Weiterhin wäre ein direkter Vergleich der Ergebnisse der Ultraschallspektroskopie mit hinreichend langen Computersimulationen nötig, um die Details bei der Rotation um die glycosidischen Bindungswinkel $\omega$ und $\psi$ zu klären.

Im Hinblick auf biologische und pharmazeutische Anwendungen sind Untersuchungen an Modellmembranen erstrebenswert, in die - entsprechend biologischer Membranen - Glycolipide und/oder Glycoproteine eingebettet sind. Hier können die Erfahrungen in der Ultraschallspektroskopie mit Lipidsystemen und Kohlenhydraten kombiniert werden. 


\section{A Spektren einiger Monosaccharide}

Im Folgenden werden die Spektren der Monosaccharide Glucose, Galactose und Fructose kurz dargestellt [21] [56] [16]. Diese drei Zucker sind die Konstituenten, aus denen die für diese Arbeit untersuchten Disaccharide aufgebaut sind. Es ist für die Analyse der Disaccharid-Spektren hilfreich, die bereits bei den Monosacchariden auftretenden Relaxationen und ihre Ursache zu betrachten, da ein Teil der molekulare Prozesse bei beiden Kohlenhydratgruppen identisch ist.

\section{A.1 Glucose}

Die folgende Abbildung A.1 zeigt die Excess-Absorption längs einer Wellenlänge einer 1-molaren wässrigen Glucoselösung. Sie zeigt zwei Relaxationsbereiche, die sich jeweils mit einer Debye-Spektralfunktion beschreiben lassen. Der tieffrequente Term $\left(, \delta^{\prime}\right)$ lässt sich der Rotation der exocyclischen Hydroxymethyl-Gruppe, der hochfrequente $\left(, \varepsilon^{*}\right)$ einem Assoziationsprozess der Glucoseringe zuordnen [62].

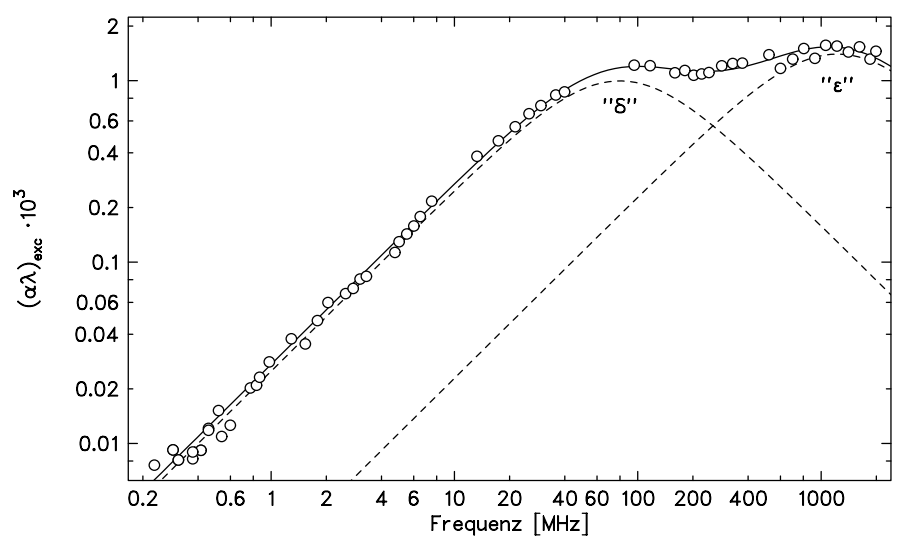

Abbildung A.1: Excess-Absorption einer 1-molaren wässrigen Glucoselösung bei $25^{\circ} \mathrm{C}$. Die gestrichelten Linien geben zwei Debye-Spektralterme, die durchgezogene deren Summe wieder.

\section{A.2 Fructose}

Das Absorptionsspektrum der Fructose zeigt drei Relaxationsbereiche. Neben den beiden hochfrequenten Debye-Termen, die auch bei der Glucose auftreten, gibt es einen zusätzlichen tieffrequenten Debye-Spektralterm (, $\left.\alpha^{\text {“ }}\right)$, der die ${ }^{2} \mathrm{C}_{5} \leftrightharpoons{ }^{5} \mathrm{C}_{2}$ 
Sessel-Sessel-Umwandlung der $\alpha$-D-Fructopyranose wiedergibt, die in einer wässrigen Fructoselösung zu ca. 2\% vorliegt (Abb. 6.10) [62]. Die folgende Abbildung A.2 zeigt die $(\alpha \lambda)_{\text {exc }}$-Darstellung des Spektrums einer 1-molaren wässrigen Lösung.

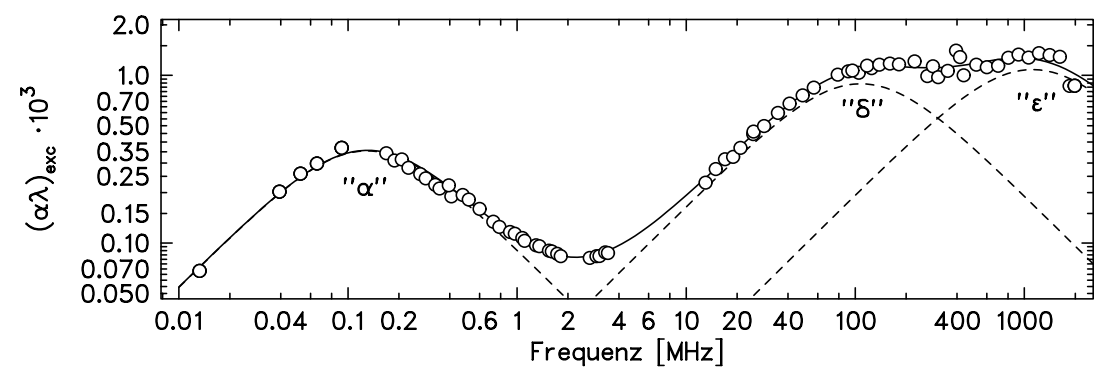

Abbildung A.2: Excess-Absorption einer 1-molaren wässrigen Fructoselösung bei $25^{\circ} \mathrm{C}$. Die gestrichelten Linien geben drei Debye-Spektralterme, die durchgezogene deren Summe wieder.

\section{A.3 Galactose}

Das Exzess-Spektrum der Galactose zeigt ebenfalls drei Relaxationsbereiche, die sich durch Debye-Spektralfunktionen beschreiben lassen. Wie die Abbildung A.3 zeigt, wird hier im hochfrequenten nur ein Debye-Term benötigt, der nach [62] der Rotation der exocyclischen $\mathrm{CH}_{2} \mathrm{OH}-$ Gruppe $\left(, \delta^{\prime}\right)$ zugeordnet wird. Wie in Abschnitt 8.2 erläutert, ist unter Berücksichtigung der Disaccharid--Spektren und von Computersimulationen die Zuordnung zu einem Assoziationvorgang („, $\epsilon^{\prime)}$ zu bevorzugen.

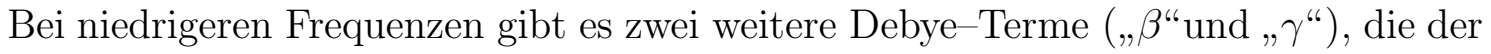
Pseudorotation des Ringes zugeordnet werden [62]. Bei der sogenannten Pseudorotation handelt es sich um keine vollständige Ringinversion wie bei der Sessel-Sessel Umwandlung, sondern um den Wechsel in Zwischenzustände, wie der verdrehten Wanne oder dem Halbsessel (vgl. Abb. 6.7).

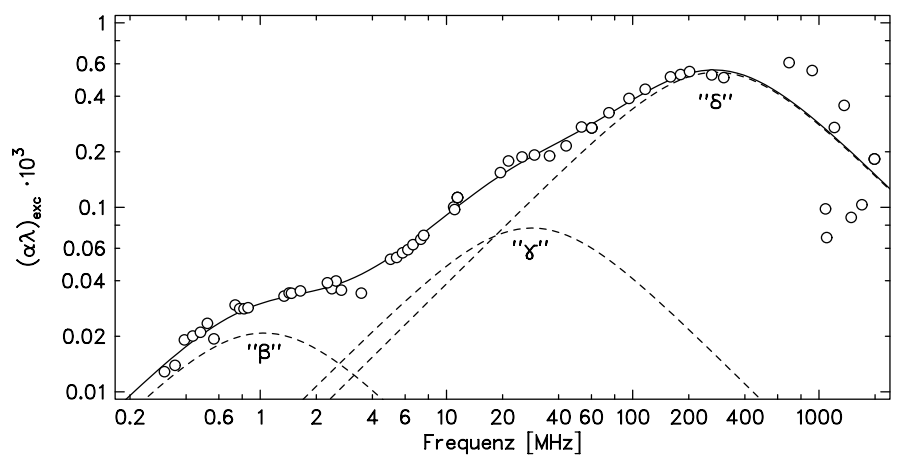

Abbildung A.3: Excess-Absorption einer 1-molaren wässrigen Galactoselösung bei $25^{\circ} \mathrm{C}$. Die gestrichelten Linien geben drei Debye-Spektralterme, die durchgezogene deren Summe wieder. 


\section{Literaturverzeichnis}

[1] Sharon N., Lis H.; Science 246 (1989) 227

[2] Dennis J.W., Laferté S., Waghorne C., Breitman M.L., Kerbel R.S.; Science 236 (1987) 582

[3] Helenius A., Aebi M.; Science 291 (2001) 2364

[4] Alper J.; Science 291 (2001) 2364

[5] Matthews G.; Nature 406 (2000) 835

[6] Lindhorst T.K.; Chemie in unserer Zeit 1 (2000) 38

[7] Sharon N., Lis H.; Spektrum der Wissenschaft 3 (1993) 66

[8] Maeder T.; Spektrum der Wissenschaft 2 (2003) 46

[9] Kaatze U.; Skript zur Vorlesung Einführung in die Biophysik II DPI (2000)

[10] Labhardt A.; Dissertation, Phil.-Nat.-Fak. Univ. Basel (1975)

[11] Behrends R.; Diplomarbeit, Mat.-Nat.-Fak. Univ. Göttingen (1994)

[12] Telgmann T.; Dissertation, Mat.-Nat.-Fak. Univ. Göttingen (1997)

[13] Telgmann T.; Diplomarbeit, Mat.-Nat.-Fak. Univ. Göttingen (1994)

[14] Lemons R.A., Quate C.F.; in: Mason W.P., Thurston R.N. (Eds.); Physical Acoustics XIV, Academic Press, New York (1979) 1

[15] Eggers F.; Acustica 76 (1992) 231

[16] Polacek R.; Diplomarbeit, Mat.-Nat.-Fak. Univ. Göttingen (1999)

[17] Meyer E., Neumann E.G.; Physikalische und Technische Akustik, Vieweg, Braunschweig (1979)

[18] Kaatze U., Lautscham K.; J. Phys. E: Sci. Instrum. 19 (1986) 1046

[19] Fay B.; Acustica 36 (1976/77) 209

[20] Menzel K.; Dissertation, Mat.-Nat.-Fak. Univ. Göttingen (1993)

[21] Riech M.; Diplomarbeit, Mat.-Nat.-Fak. Univ. Göttingen (1996) 
[22] Kühnel V.; Dissertation, Mat.-Nat.-Fak. Univ. Göttingen (1995)

[23] Lautscham K.; Dissertation, Mat.-Nat.-Fak. Univ. Göttingen (1986)

[24] Kaatze U., Lautscham K., Brai M.; J. Phys. E: Sci. Instrum. 21 (1988) 98

[25] Kaatze U., Kühnel V., Menzel K., Schwerdtfeger S.; Meas. Sci. Technol. 4 (1993) 1257

[26] Kaatze U., Kühnel V., Weiss G.; Ultrasonics 34 (1996) 51

[27] Rupprecht A., Dissertation, Mat.-Nat.-Fak. Univ. Göttingen (1997)

[28] Gailus T.; Dissertation, Mat.-Nat.-Fak. Univ. Göttingen (1996)

[29] Bömmel H.E., Dransfeld K.; Phys. Rev. Lett. 1 (1958) 234

[30] Hagen R., Diplomarbeit, Mat.-Nat.-Fak. Univ. Göttingen (1998)

[31] National Institute of Standards and Technology; NIST Chemistry WebBook; Thermophysical Properties of Fluid Systems; www.nist.gov

[32] Rupprecht A., Kaatze U.; J. Phys. Chem. A 106 (2002) 8850

[33] Bödecker G.; Diplomarbeit, Mat.-Nat.-Fak. Univ. Göttingen (1974)

[34] Grdadolnik J.; Maréchal Y.; J. Mol. Struc. 615 (2002)

[35] Douhéret G.; Davis M.I.; Reis J.C.R.; Blandamer M.J.; Chem. Phys. Chem. 2 (2001)

[36] Lehmann J.; Kohlenhydrate, Georg Thieme Verlag, Stuttgart (1996)

[37] Ernst B., Hart G.W., Sinä̈ P.; Carbohydrates in Chemistry and Biology, WileyVCH Verlag, Weinheim (2000)

[38] Beyer H.; Lehrbuch der Organischen Chemie, Hirzel Verlag, Stuttgart (1998)

[39] Mortimer C.E.; Chemie, Georg Thieme Verlag, Stuttgart (1987)

[40] Bhatia A.B.; Ultrasonic Absorption, Oxford University Press (1967)

[41] Strehlow H.; Rapid Reactions in Solutions, VCH Verlagsgesellschaft, Weinheim (1992)

[42] Herzfeld K.F., Litovitz T.A.; Absorption and Dispersion of Ultrasonic Waves, Academic Press, New York London (1959)

[43] Menzel K., Rupprecht A., Kaatze U.; J. Acoust. Soc. Am. 104 (1998) 2741

[44] Giese K.; Adv. Mol. Relaxation Processes 5 (1973) 363

[45] Marquardt D.W.; J. Soc. Indust. Appl. Math. 2 (1963) 2

[46] Weber M.; Turbo Pascal Tools, Vieweg, Braunschweig (1989) 244 
[47] Rassing J., Wyn-Jones E.; Chem. Phys. Lett. 21 (1973) 93

[48] Madigosky W.M., Warfield R.W.; Acustica 55 (1984) 123

[49] Telgmann T., Kaatze U.; J. Phys. Chem. 101 (1997) 7758

[50] Romanov V.P., Solovjev V.A.; Sov. Phys. Acoust. 11 (1965) 68, 219

[51] Montrose C.J., Litovitz T.A.; J. Acoust. Soc. Am. 47 (1970) 1250

[52] Endo H.; J. Chem. Phys. 92 (1990) 1986

[53] Ornstein L.S., Zernike F.; Phys. Z. 19 (1918) 134

[54] Brant D.A.; in: Preiss J. (Ed.); The Biochemistry of Plants, Academic Press, New York (1980) 425

[55] Branca C., Magazù S., Maisano G., Migliardo P.; J. Chem. Phys. 111 (1999) 281

[56] Stenger J.; Diplomarbeit, Mat.-Nat.-Fak. Univ. Göttingen (1998)

[57] Baucke E.; Staatsexamensarbeit, Mat.-Nat.-Fak. Univ. Göttingen (2002)

[58] Cowman M., Eggers F., Eyring E.M., Horoszewski D., Kaatze U., Kreitner R., Petrucci S., Klöppel-Riech M., Stenger J.; J. Phys. Chem. B 103 (1999) 239

[59] Behrends R., Cowman M., Eggers F., Eyring E., Edward M., Kaatze U., Majewski J., Petrucci S., Richmann K., Riech M.; J. Am. Chem. Soc. 119 (1997) 2182

[60] Stenger J., Cowman M., Eggers F., Eyring E.M., Kaatze U., Petrucci S.; J. Phys. Chem. B 104 (2000) 4782

[61] Polacek R., Behrends R., Kaatze U.; J. Phys. Chem. B 105 (2001) 2894

[62] Polacek R., Stenger J., Kaatze U.; J. Chem. Phys. 116(7) (2002) 2973

[63] Ott K.H., Meyer B.; Carbohydr. Res. 281 (1996) 11

[64] Kirschner K.N., Woods R.J.; Proc. Nat. Acad. Sci. 98 (2001) 10541

[65] Eklund R., Widmalm G.; Carbohydr. Res. 338 (2003) 393

[66] Frank M.; Dissertation, Nat.-Mat. Gesamtfak. Univ. Heidelberg (2000)

[67] Tvaroska I, Taravel F.R., Utille J.P., Carver J.P.; Carbohydr. Res. 337 (2002) 353

[68] Bernardi A., Raimondi L., Zanferrari D.; J. Mol. Struct. (Theochem) 395\{396 (1997) 361

[69] Qasba P.K., Balaji P.V., Rao V.S.R.; J. Mol. Struct. (Theochem) 395\{396 (1997) 333 
[70] Nishida Y., Ohrui H., Meguro H.; Tetrahedron Letters 25 (1984) 1575

[71] Kühnel V; Diplomarbeit, Mat.-Nat.-Fak. Univ. Göttingen (1991)

[72] Behrends R.; Dissertation, Mat.-Nat.-Fak. Univ. Göttingen (1999)

[73] Behrends R.; Unveröffentlichte Messung DPI (2003)

[74] Fuchs K., Kaatze U.; J. Chem. Phys. 116(16) (2002) 7137

[75] Atkins P.W.; Physikalische Chemie, VCH, Weinheim (1990)

[76] Behrends R.; Skript zur Vorlesung Grundlagen der molekularen Flüssigkeitsphysik II DPI (2003)

[77] Angyal S.J.; Adv. Carbohyd. Chem. Biochem. 47 (1989) 1

[78] Franks F.; Water, A Comprehensive Treatise, Vol. 4, Plenum Press, New York (1975) 1 


\section{Danksagung}

Herrn Prof. Dr. Dirk Ronneberger danke ich für die Betreuung dieser Arbeit.

Bei Herrn Prof. Dr. Götz Eckold bedanke ich mich für die Übernahme des Korreferats.

Herrn Dr. Udo Kaatze gilt mein Dank für sein großes Interesse an der Entwicklung dieser Dissertation, die intensive Betreuung und ständige Gesprächsbereitschaft, aus der sich viele wertvolle Hinweise, Anregungen und Ratschläge ergaben.

Bei Dr. Ralph Behrends, Dr. Rüdiger Polacek, Etienne Baucke, Kerstin Fuchs, Maximilian Schach, Dr. Jens Stenger, Dr. Michael Klöppel-Riech bedanke ich mich für die zur Verfügung gestellten Messdaten und die vielen Anregungen zu Ultraschallmessungen an Kohlenhydraten. Herrn Behrends danke ich zudem für seine Bereitschaft, mir bei Fragen zu Theorie und Praxis der Ultraschallspektroskopie mit Rat und Tat zur Seite zu stehen und für die Messung des Scherimpedanzspektrums.

Bei allen aktiven und vielen mittlerweile ehemaligen Mitarbeiterinnen und Mitarbeitern der Arbeitsgruppe Kaatze möchte ich mich für die konstruktiven Diskussionen und die warme herzliche Arbeitsatmosphäre bedanken. Es war eine schöne Zeit am DPI.

Kerstin Fuchs, Steffen Zeugner, Barbara Eberhardt und David Oliczka danke ich für die sorgfältige Bestimmung der Dichten und Scherviskositäten.

Bei Herrn Dieter Hille und den Mitarbeitern der Feinmechanischen Werkstatt sowie bei Herrn Dr. Karl Lautscham und den Mitarbeitern der Elektronischen Werkstatt bedanke ich mich für ihren unermüdlichen Einsatz bei der Entwicklung, Wartung und Reparatur der Messapparaturen.

Elke Hanke, Markus Jäger und Dr. Ralph Behrends danke ich für das sorgfältige Korrekturlesen des Entwurfs zu dieser Arbeit.

Mein besonderer Dank gilt meinen Eltern für deren Unterstützung während meines gesamten Studiums. Ohne sie wäre die Anfertigung dieser Arbeit nicht möglich gewesen.

Abschließend möchte ich mich bei der Deutschen Forschungsgemeinschaft für die finanzielle Unterstützung bedanken. 


\section{Lebenslauf}

Name:
Geburtsdatum:
Geburtsort:
Staatsangehörigkeit:
Familienstand:
1978-1982
$1982-1984$
$1984-1991$
1991
1991
1993
1993
1994
1995

1995

WS 1996/1997

WS 1997/1998

SS 1998

1998

WS 1998/1999

1999

Mai 2003
Ralf Hagen

4. September 1971

Uelzen

deutsch

ledig

Grundschule Küsten

Orientierungsstufe Lüchow

Gymnasium Lüchow

Abitur

Beginn des Studiums der Physik und Mathematik an der Georg-August-Universität Göttingen

Allgemeines Schulpraktikum an der Heinrich-SohnreyRealschule Hann. Münden

Diplomvorprüfung Physik

Zwischenprüfung Mathematik

Fachpraktikum im Unterrichtsfach Physik am ErnstMoritz-Arndt-Gymnasium Herzberg/Harz

Betriebspraktikum bei der Artesan Pharma GmbH Lüchow

Studentische Hilfskraft an der Fakultät für Agrarwissenschaften. Leitung der Übungen zu den Vorlesungen „Mathematik und Statistik I \& II".

Diplomhauptprüfung Physik. Die Diplomarbeit habe ich am Dritten Physikalischen Institut in der Arbeitsgruppe Komplexe Fluide von Dr. U. Kaatze unter Betreuung von Herrn Prof. Dr. D. Ronneberger mit dem Thema „Breitbandige Ultraschallabsorptionsspektroskopie an wäßrigen n-Hexylammoniumchlorid-Lösungen im Frequenzbereich von $180 \mathrm{kHz}$ bis 4,6 GHz" angefertigt.

Lehrauftrag „Mathematik und Statistik“ an der Fakultät für Agrarwissenschaften.

Beginn der Dissertation in der Arbeitsgruppe Komplexe Fluide des Dritten Physikalischen Instituts unter Betreuung von Herrn Dr. U. Kaatze und Herrn Prof. Dr. D. Ronneberger mit dem Thema „Breitbandige Ultraschallabsorptionsspektroskopie an wässrigen Kohlenhydrat-Lösungen“. Studienreferendar am Studienseminar Celle für das Lehramt an Gymnasien. 